\title{
Loss and risk aversion in games and decisions
}

Citation for published version (APA):

Driesen, B. W. I. (2010). Loss and risk aversion in games and decisions. [Doctoral Thesis, Maastricht University]. Universitaire Press Maastricht. https://doi.org/10.26481/dis.20100429bd

Document status and date:

Published: 01/01/2010

DOI:

10.26481/dis.20100429bd

Document Version:

Publisher's PDF, also known as Version of record

\section{Please check the document version of this publication:}

- A submitted manuscript is the version of the article upon submission and before peer-review. There can be important differences between the submitted version and the official published version of record.

People interested in the research are advised to contact the author for the final version of the publication, or visit the DOI to the publisher's website.

- The final author version and the galley proof are versions of the publication after peer review.

- The final published version features the final layout of the paper including the volume, issue and page numbers.

Link to publication

\footnotetext{
General rights rights.

- You may freely distribute the URL identifying the publication in the public portal. please follow below link for the End User Agreement:

www.umlib.nl/taverne-license

Take down policy

If you believe that this document breaches copyright please contact us at:

repository@maastrichtuniversity.nl

providing details and we will investigate your claim.
}

Copyright and moral rights for the publications made accessible in the public portal are retained by the authors and/or other copyright owners and it is a condition of accessing publications that users recognise and abide by the legal requirements associated with these

- Users may download and print one copy of any publication from the public portal for the purpose of private study or research.

- You may not further distribute the material or use it for any profit-making activity or commercial gain

If the publication is distributed under the terms of Article $25 \mathrm{fa}$ of the Dutch Copyright Act, indicated by the "Taverne" license above, 


\title{
Loss and Risk Aversion in Games and Decisions
}

\author{
Bram Driesen
}


(C) Bram Driesen, 2010

All rights reserved. No part of this publication may be reproduced, stored in a retrieval system, or transmitted in any form, or by any means, electronic, mechanical, photocopying, recording or otherwise, without the prior permission in writing from the author.

This book was typeset by the author using $\mathrm{IATEX}_{\mathrm{E}}$.

Published by

Universitaire Pers Maastricht

ISBN: 978-90-5278-934-7

Printed in The Netherlands by Datawyse Maastricht 


\title{
Loss and Risk Aversion in Games and Decisions
}

\author{
Proefschrift
}

ter verkrijging van de graad van doctor aan de Universiteit Maastricht, op gezag van Rector Magnificus, Prof. mr. G.P.M.F. Mols, volgens het besluit van het College van Decanen, in het openbaar te verdedigen op donderdag 29 april 2010 om 12:00 uur

door

\section{Bram Driesen}

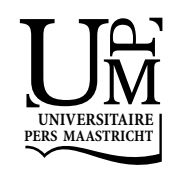




\section{Promotores}

Prof. dr. H. J. M. Peters

Prof. dr. P. P. Wakker

\section{Copromotor}

Dr. ir. A. Perea y Monsuwé

\section{Beoordelingscommissie}

Prof. dr. A. Riedl (voorzitter)

Prof. dr. B. Klaus

Dr. A. J. A. Storcken

Dit onderzoek werd financieel mogelijk gemaakt door NWO [400 - 04 - 100]. 


\section{Acknowledgments}

In your hands you hold the fruits of my labor of these last four years: my dissertation "Loss and Risk Aversion in Games and Decisions". Though the cover only bears my name, I was by no means the only one responsible for its realization. I am indebted to a great many people who have in one way or another contributed to the writing of these pages, and I would like to take the current opportunity to gratefully acknowledge some of them.

In the first place, I would like to thank Hans and Andrés, who took on the supervision of the game theory parts of this thesis. As it happens, Hans and Andrés were also the ones who first introduced me to this field. It was their course "Game Theory and Economics", which I took in the second year of my undergraduate studies, that sparked my first interest in game theory, and can thus be considered the very foundation of this thesis. In the capacity of supervisors, Hans and Andrés have of course also contributed in a much more direct way. Over the years we have had many, many discussions about the contents of this work, something which has obviously benefited its quality tremendously. I owe them many thanks for all their support, advice, and guidance.

Peter was responsible for the supervision of the decision theoretic part of this dissertation. We started out working together but unfortunately, somewhere along the way my personal interests seem to have gradually led me to subjects outside of Peter's area of expertise. Nevertheless, I owe him thanks for giving me the opportunity to co-author with him and Aurélien Baillon, and express the hope of a continued collaboration in the future.

Undertaking research can at times be difficult and frustrating, especially in the periods when efforts are not rewarded with results. Nonetheless, I 
tend to associate my doctoral period at this university as much with snowboarding, soccer, bowling, board games, dinner parties, and the likes, than I do with the hardships of $\mathrm{PhD}$ research. Without going into the specifics of names, I am thankful to the many friends and colleagues - past and present - for making this 'life besides research' so great.

A special word of thanks goes to my parents, Elizabeth and Leon. The gratitude I owe them for all their encouragement, support, and pride is probably too often left to the understanding, so let me conclude by explicitly expressing it here: Thanks a million bucks!

Bram Driesen

Maastricht, January 2010 


\section{Contents}

$\begin{array}{ll}\text { Acknowledgments } & 5\end{array}$

1 Introduction $\quad 11$

1.1 Loss Aversion in Strategic Interactions . . . . . . . . . . . 13

1.1.1 The Rubinstein Bargaining Game . . . . . . . . . . 14

1.1.2 The Bargaining Problem . . . . . . . . . . . . . . 16

1.1.3 Loss Aversion Equilibria . . . . . . . . . . . . . . . . 17

1.2 A Comparative Tool for Risk and Ambiguity . . . . . . . . 18

1.3 Fictitious Play: A Convergence Result . . . . . . . . . . 18

2 Alternating Offers Bargaining with Loss Aversion 21

2.1 Introduction . . . . . . . . . . . . . . . . . 21

2.2 The Alternating Offers Bargaining Model with Loss Aversion . 24

2.3 A Subgame Perfect Equilibrium . . . . . . . . . . . . . 26

2.4 A Uniqueness Result . . . . . . . . . . . . . . . . . . . . 28

2.5 Analysis of the Equilibrium . . . . . . . . . . 30

2.5.1 Comparative Statics of the Loss Aversion Coefficients . 30

2.5.2 Convergence Results with respect to the Probability of Continuation ................ 31

2.6 Concluding Remarks . . . . . . . . . . . . . . . . . . . 34

2.A Appendix: Proofs . . . . . . . . . . . . . . . . . 34

2.A.1 The Formal Model and the SPE . . . . . . . . . . . 34

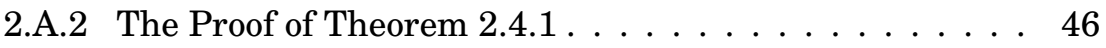

2.A.3 Concave Utility Functions . . . . . . . . . . . . . 61 
3 The Kalai-Smorodinsky Bargaining Solution with Loss Aversion

3.1 Introduction . . . . . . . . . . . . . . . . 65

3.2 Preliminaries . . . . . . . . . . . . . . . 67

3.3 Bargaining with Loss Aversion . . . . . . . . . . . . . 68

3.4 The Kalai-Smorodinsky Bargaining Solution . . . . . . . . . 70

3.5 The Solution Class $\mathscr{D}^{N} \ldots \ldots \ldots \ldots \ldots \ldots$

3.6 An Axiomatic Characterization of $\mathscr{D}^{N} \ldots \ldots \ldots \ldots 73$

3.7 Concluding Remarks . . . . . . . . . . . . . . . . . 75

3.A Appendix: Proofs . . . . . . . . . . . . . . . 76

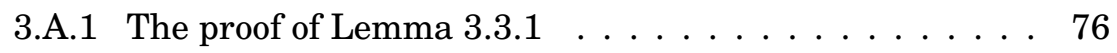

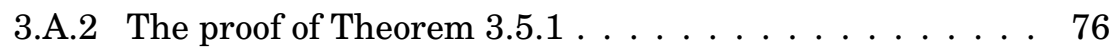

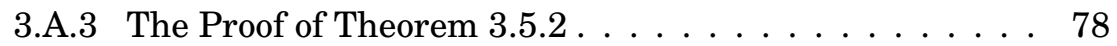

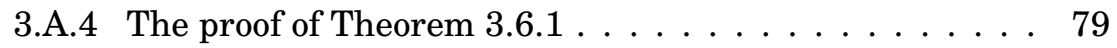

4 On Loss Aversion in Bimatrix Games $\quad 83$

4.1 Introduction . . . . . . . . . . . . . . 83

4.2 Preliminaries . . . . . . . . . . . . . . 86

4.2.1 Bimatrix Games and Nash Equilibrium . . . . . . . 86

4.2 .2 Loss Aversion . . . . . . . . . . . . . . . . . 86

4.3 Fixed Point Loss Aversion Equilibrium . . . . . . . . . . . . . 87

4.4 Maximin Loss Aversion Equilibrium . . . . . . . . . . . . . . 88

4.4.1 Definition of Maximin Loss Aversion Equilibrium . . . . 89

4.4.2 Existence of Maximin Loss Aversion Equilibrium . . . . 90

4.5 Safety Level Loss Aversion Equilibria . . . . . . . . . . . . . 93

4.5.1 Definition and Existence of Safety Level Loss Aversion Equilibria . . . . . . . . . . . . . . 94

4.5.2 Strict Dominance when Players are Loss Averse . . . . . 95

4.6 Comparative Statics . . . . . . . . . . . . . . . . . 98

4.6 .1 Preliminaries . . . . . . . . . . . . . . 98

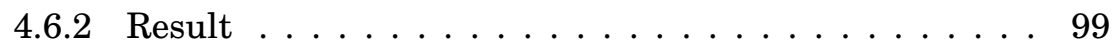

4.7 Summary . . . . . . . . . . . . . . . . 100

4.A Appendix: Proofs . . . . . . . . . . . . . . 100

4.A.1 The Proof of Theorem 4.6.1 . . . . . . . . . . . 100

5 Relative Concave Utility for Risk and Ambiguity 105

5.1 Introduction . . . . . . . . . . . . . . . . . 105

5.2 Risk Aversion through Marginal Rates of Substitution . . . . 107

5.3 Elementary Definitions . . . . . . . . . . . . . . . . . 109 
5.4 Technical Definitions $\ldots \ldots \ldots \ldots$

5.5 Formalizing Rates of Substitution . . . . . . . . . . . . . . . 112

5.5.1 Rates of Substitution Defined Formally . . . . . . . . 112

5.5.2 Measuring Utility using Rates of Substitution . . . . . . 113

5.5.3 Preference Foundations . . . . . . . . . . . . . . . . . . 114

5.5.4 Risk Aversion Analyzed using DRS . . . . . . . . . . . . . 116

5.6 Yaari's Comparative Risk Aversion Results . . . . . . . . . . 117

5.7 Recursive Models . . . . . . . . . . . . . . . . . . . . . . . 118

5.8 Comparative Results for Recursive Models . . . . . . . . . . . 122

5.9 Alternative Recursive Models . . . . . . . . . . . . . . . . . . 124

5.10 Discussion . . . . . . . . . . . . . . . . . . . 128

5.11 Concluding Remarks . . . . . . . . . . . . . . . . . 129

5.A Appendix: Proofs . . . . . . . . . . . . . . . . . 129

6 Continuous Fictitious Play in Zero-Sum Games 133

6.1 Introduction . . . . . . . . . . . . . . . . 133

6.2 Preliminaries . . . . . . . . . . . . . . . . . 134

6.2.1 Zero-sum Games . . . . . . . . . . . . . . . . . . . 134

6.2.2 Continuous Fictitious Play . . . . . . . . . . . . 135

6.3 CFP Convergence in Zero-Sum Games . . . . . . . . . . . . 136

6.4 DFP in Zero-Sum Games . . . . . . . . . . . . . . . 138

$\begin{array}{lr}\text { Bibliography } & 139\end{array}$

$\begin{array}{ll}\text { Nederlandse Samenvatting } & 147\end{array}$

$\begin{array}{ll}\text { Curriculum Vitae } & 153\end{array}$ 



\section{Chapter 1}

\section{Introduction}

This thesis deals with topics in decision theory, game theory, and the interface between these two disciplines. The subfield of decision theory under consideration in the present text, decision under uncertainty, examines how individuals make decisions and choices in an uncertain world, and how these choices are affected by the behavioral constitution of the decision maker. This is best illustrated by a classic example of Bernoulli (1738), one of the founding fathers of the field. Consider a lottery ticket that with equal probability yields either $€ 20,000$, or $€ 0^{1}$. The question Bernoulli asked is at what price one should sell such a ticket. That is, what sure amount of money would its owner consider an adequate remuneration for the chance of gaining $€ 20,000$, and the risk of ending up with nothing? Building further on this example, one might wonder how the decision at what price to sell changes if we are not considering a lottery ticket, but rather a bet of $€$ 20,000 on a tennis match between two unknown players. And how would things change if one is not the seller of such a ticket, but the buyer? That is, at what price should one buy such a ticket?

While these questions may appear academic at first, their importance reaches far beyond the realm of lotteries and tennis matches. Should I get insurance against bicycle theft? Should I take the car to work, knowing that the bus is slower but safer? Should I stop smoking today, knowing that this may keep me from health problems thirty years from now? These are but a few examples taken from the abundance of decision problems we face all the time. By investigating problems like Bernoulli's, decision theory aims to unveil the systems underlying the choices we make when presented with

\footnotetext{
${ }^{1}$ The currency in Bernoulli's example is 'ducates'.
} 
these predicaments. Not surprisingly, the study of decision making is at the heart of many social sciences such as psychology, sociology, and economics.

Decision under uncertainty is limited to the study of choice problems for isolated individuals. This is only part of a bigger story. Especially in the context of economics, we do not simply operate as isolated elements in an empty space. We trade, we bargain, we compete and we collude - in short, we interact. The outcome of a decision made by an economic agent is not only contingent on what state of the world materializes - winning the lottery/not winning the lottery, tennis player A wins/tennis player B wins - but also on the actions taken by one or more other agents who are faced with the same problem: making the decision that is in their best interest, with the understanding that the outcome depends on the combination of all strategies played, the others' as well as their own. Such strategic interactions are the topic of study in the field of game theory. Ethymologically, this term can be traced back to the works of John von Neumann (1928) and Ernst Zermelo (1913) - pure mathematicians who independently applied quantitative analysis to the play of parlor games. By today's standards, however, the name 'game theory' may be too restrictive. Firms competing for market share, species fighting for survival, bidders trying to secure the object of an auction with the lowest possible bid, haggling down a price: all these are instances of games. One should not let the frivolous connotation of that term obfuscate the true extent of its present definition.

While game theory has a meaningful use in several scientific disciplines, it is hard to think of any in which it has taken as prominent a place as in economics. Aside from a couple of stray exceptions, its application to economic theory began with the publication of von Neumann and Morgenstern's (1944) landmark book Theory of Games and Economic Behavior, in which they achieved a highly elegant integration of the principles of decision making put forth by Bernoulli (1738), with the game theoretic concepts prevalent at the time. The importance of game theory for economics would become even greater a few years later, when John Nash (1950b) proved the famous result that each game has at least one equilibrium ${ }^{2}$.

In spite of its impressive elegance, the expected utility framework used by von Neumann and Morgenstern did not remain without criticism. Already in

\footnotetext{
${ }^{2}$ What Nash called an equilibrium is known today as a Nash equilibrium. Roughly speaking, a Nash equilibrium is a strategy profile with the property that each player maximizes his expected payoff by following his own equilibrium strategy, given that all others do the same.
} 
the early fifties, Allais (1953) conducted an experiment that revealed that individuals make choices which are not consistent with that framework. Over the years, more paradoxes and puzzles appeared that question the expected utility paradigm, making the call for an alternative decision model louder and louder. A serious contender - named prospect theory - was developed by Kahneman and Tversky (1979). They retained the basic structure of the expected utility model, but enriched it with a number of behavioral aspects to individual decision making, abundantly documented in psychology literature of the time. An important feature captured by prospect theory is reference dependence, leading to loss aversion. Loss aversion is based on the premise that losses are considered to be more important than gains. However obvious this claim may seem, until the groundbreaking work of Kahneman and Tversky, the idea that the upset of receiving a utility of four when one of five was expected, tends to exceed the joy of receiving one of six under that same expectation, managed to elude economists for ages.

Given that Kahneman and Tversky's decision model specifically takes into account a number of obvious behavioral quirks, it comes as little surprise that it significantly outperforms its expected utility counterpart. This success for the case of individual decision making suggests that the integration of prospect theory with game theory will likewise lead to better models for the analysis of strategic interactions. However, much of the beautiful fit of expected utility with the analysis of games, seems to evaporate once we impose Kahneman and Tversky's extenstions to the expected utility model. Indeed, in spite of the obvious appeal of an integrated theory, attempts to enrich game theoric concepts with elements of prospect theory raise a multitude of nontrivial questions and problems. These are the waters we sail in the next three chapters. In particular, we investigate the implications of loss aversion for a selection of well known game theoretic results.

\subsection{Loss Aversion in Strategic Interactions}

To model loss aversion we adopt the simple approach of Shalev (2000). He assumed that each player has in mind some utility level $r$ - an expectation or an aspiration - which separates his spectrum of utility payoffs into gains and losses. Troughout this thesis, this utility level is referred to as the player's reference point. Tversky and Kahneman (1981) pointed out that any utility outcome $x$ can be expressed as a deviation from that reference point: $x=$ $r+(x-r)$. Shalev captured Kahneman and Tversky's (1979) hypothesis that 
negative deviations $(x-r<0)$ are more important than positive ones $(x-r \geq$ 0 ) with the simple, but elegant transformation

$$
U(x)= \begin{cases}r+(x-r) & \text { if } x-r \geq 0 \\ r+(1+\lambda) \cdot(x-r) & \text { if } x-r<0\end{cases}
$$

where the positive parameter $\lambda$ is a measure of the agent's loss aversion. At first glance, introducing this single effect to games hardly seems like a challenging task. After all, one can simply apply von Neumann and Morgenstern's framework to the game, after correcting for players' loss aversion with Shalev's transformation. In spite of the apparent simplicity of the problem, one quickly stumbles on a highly nontrivial issue: what should be the players' reference points? What do they consider gains and what losses? The road usually taken in the literature is to exogenously specify some utility level that somehow seems suitable to the situation. We learn from Tversky and Kahneman (1981) p. 453, that such an approach may not be entirely acceptable.

A decision problem is defined by the acts or options among which one must choose, the possible outcomes or consequences of these acts, and the contingencies or conditional probabilities that relate outcomes to acts. We use the term "decision frame" to refer to the decision-maker's conception of the acts, outcomes, and contingencies associated with a particular choice.

Whether a player considers a payoff to be a gain or a loss, can be assumed to depend on his decision frame. In a game theoretic context, this implies that a player's reference point depends on the strategies he has at his disposal [the acts], the utility payoffs associated with these strategies [the outcomes], and - crucially - the strategies played by his opponents [the contingencies]. It follows that, in order to correctly integrate loss aversion with the theory of games, determining players' reference points should be taken as an inherent part of the solution concept they choose to adopt.

\subsubsection{The Rubinstein Bargaining Game}

Chapter 2 reconsiders Rubinstein's (1982) alternating offers bargaining game under the assumption that bargainers are loss averse. In the classical conception of this game, two bargainers have to divide a perfectly divisible unit 
good. They do so by alternatingly proposing to one another some division of that good, a procedure that continues until one makes an offer which is accepted by the other. In this case bargaining ends, and players obtain the utility payoffs implied by the accepted proposal. Rejecting a proposal is costly in the sense that it is assumed to lead with a small, but strictly positive probability, to a breakdown of the negotiations, leaving both players with a zero payoff ${ }^{3}$. Without going into the specifics, Rubinstein constructed a Subgame Perfect Equilibrium (SPE) in this game, and proved that it was the only such equilibrium.

In the current setup, each bargainer is assumed to be loss averse, and the associated reference point is equal to the highest turned down offer in the past. This implies a nontrivial extension of Rubinstein's original model in the sense that payoffs, and therefore also the potential equilibrium strategies, become dependent on the history of play. As a first major result, we construct an SPE, in which the strategies depend on the history of play through the current reference points. The second major result of this chapter is a characterization of this equilibrium. In particular, it is shown that it is the unique one under three assumptions shared with the classical Rubinstein model:

- immediate acceptance of equilibrium offers;

- indifference between acceptance and rejection of such offers;

- strategies only depend on the current set of reference points, and not on the past play of the game.

While this characterization does not exclude the possibility that other equilibria exist, it does show that our SPE is the only one similar to Rubinstein's. It is furthermore shown that in this specific equilibrium, being loss averse is a disadvantage.

Binmore, Rubinstein, and Wolinsky (1986) showed that the unique SPE of the classical alternating offers bargaining model results in the Nash bargaining solution (Nash 1950a) outcomes if the probability of breakdown approaches zero. We establish a similar relation between our equilibrium and asymmetric Nash bargaining solutions (Harsanyi and Selten 1972; Kalai 1977), where each player's bargaining power is negatively related to own loss aversion and positively to the opponent's.

\footnotetext{
${ }^{3}$ Rubinstein (1982) adopted the equivalent setup of time discounting, where after each rejection, the good becomes slightly less valuable.
} 


\subsubsection{The Bargaining Problem}

In Chapter 3, we turn to Nash' (1950a) static, 'one-shot' bargaining problem. There, players have to cooperatively agree on an outcome, knowing that failure to reach agreement leads to an unfavorable outcome for all. To introduce loss aversion into this setting, we impose Shalev's (2002) condition of self-support. Each bargainer who has full information about the bargaining game, the bargaining solution to be used, and the opponents' attitudes towards incurring losses, will form some expectation or aspiration about the payoff he can realize. The fact that payoffs below this reference point are considered losses and are thus transformed accordingly, implies that the bargaining problem depends on the a priori expectations of the players. The condition of self-support then imposes that the payoffs players realize ex post - using the agreed upon bargaining solution in the transformed game - are consistent with those ex ante expectations. If an outcome satisfies this condition for a certain bargaining solution, it is said to be self-supporting under that bargaining solution.

The idea just described is applied to the $n$-player Kalai-Smorodinsky solution (KS) (Kalai, 1977; Peters and Tijs, 1984). The main result of the chapter is that each game has exactly one outcome that is self-supporting under KS. Since this is necessarily an untransformed element of the bargaining set, there must exist a bargaining solution that yields exactly this outcome. We explicitly define this bargaining solution. More specifically, we define a new class of bargaining solutions, such that each loss aversion profile of the players is associated with a single solution in this class; this solution then yields the unique outcome that is self-supporting under KS for the prevalent loss aversion profile. These bargaining solutions can be interpreted as asymmetric versions of KS, where the introduced asymmetry in a sense 'corrects' for the varying loss attitudes of the players.

The chapter concludes with an axiomatic characterization of this newly defined solution class. A bargaining solution is in our class if and only if it satisfies the axioms of Scale Invariance [SI], Individual Monotonicity [IM], and Strong Individual Rationality [SIR], and a new axiom called Proportional Concession Invariance [PCI]. Roughly speaking, a bargaining solution satisfies PCI if moving the utopia point in the direction of the solution outcome does not change this outcome. The traditional axiom of Pareto $\mathrm{Op}$ timality [PO] is not included in the axiomatization, as it is implied by these four properties. 


\subsubsection{Loss Aversion Equilibria}

In this chapter we turn to bimatrix games. In this setting, we study three different types of loss aversion equilibrium. A loss aversion equilibrium is a Nash equilibrium of a game in which the players are assumed to be loss averse, and where - consistent with the framing concept put forth by Tversky and Kahneman (1981) - reference points are made endogenous to the equilibrium calculation.

The first type under consideration is the fixed point loss aversion equilibrium (Shalev 2000). In this equilibrium concept, players' reference points depend on the beliefs they have about the strategies of their opponents. A second type of loss aversion equilibrium, named maximin loss aversion equilibrium, differs from fixed point loss aversion equilibrium in that each player's reference point is based on the support of his opponent's equilibrium strategy, but no longer on the exact probabilities. In the third type, called safety level loss aversion equilibrium, the reference points depend on the values of the own payoff matrices.

The introduction of new equilibrium concepts raises an important question. Can we always find such an equilibrium? For a fixed point loss aversion equilibrium, Shalev (2002) already answered this question in the affirmative, even for games with more than two players. A first result of this chapter is that for maximin loss aversion equilibria, existence cannot be guaranteed. By means of an example, we uncover the underlying reason for this negative result: the best-reply correspondence, associated with this equilibrium concept, violates upper semi-continuity. Nonetheless, general existence of maximin loss aversion equilibrium is established for the subclass of $m \times 2$ and $2 \times n$ bimatrix games. The general existence of safety level loss aversion equilibria is obtained by means of a straightforward fixed point argument.

The chapter concludes with a comparative statics analysis of all three equilibrium concepts in the case of $2 \times 2$ bimatrix games. In the bargaining games considered earlier, it is unambiguously true that loss aversion is a disadvantage. The comparative statics analysis of this chapter reveals that for bimatrix games, this is not the case. Nonetheless, a condition is obtained under which a player benefits from his opponent erroneously believing that he (the player) is more averse to losses than he actually is. 


\subsection{A Comparative Tool for Risk and Ambiguity}

Chapter 5 of this dissertation deals with aversion to risk and ambiguity. It is well known that the Expected Utility model equates the concavity of an agent's utility function with that agent's degree of risk aversion. We present a general technique that allows for comparing the concavity of utility functions - and thus risk attitudes - that requires no explicit knowledge of events or probabilities. This technique, named the preference midpoint technique, makes use of the close link between consumer theory and decision under uncertainty that follows from Arrow's (1964) and Debreu's (1959) observation that amounts of a certain good can be reinterpreted as the utility payoffs associated with a certain contingency. In particular, the preference midpoint technique makes use of rates of substitution, well known from consumer theory, to analyze preferences under uncertainty. While elementary in a mathematical sense, this technique is new and implies several generalizations of classical results:

(a) Yaari's between-person comparisons of risk aversion without requiring identical beliefs;

(b) Kreps \& Porteus' information-timing preference without requiring known probabilities, commitment to backward induction, or violation of reduction of compound lotteries;

(c) Klibanoff, Marinacci, \& Mukerji's smooth ambiguity aversion without using subjective probabilities (which are not directly observable) as inputs in preference conditions and, again, without requiring backward induction or violation of reduction of compound lotteries;

(d) Comparative smooth ambiguity aversion without the requirement that second-order subjective probabilities be identical.

Because our technique completely isolates the empirical meaning of utility, it sheds new light on the descriptive appropriateness of utility to model risk and ambiguity attitudes.

\subsection{Fictitious Play: A Convergence Result}

The thesis concludes with a purely game theoretic result, unrelated to loss or risk aversion. In particular, it provides an alternative proof for the well 
known fact that two-person zero-sum games have the Fictitious Play Property [FPP]. Fictitious Play [FP] is a learning process that belongs to the literature aiming to justify the Nash equilibrium concept using the argument that boundedly rational players can learn to play equilibrium. In the specific case of FP, each player myopically plays the strategy that yields him the highest expected utility payoff, given the empirical past play of his opponent. While updating is frequently taken to occur at discrete points in time, FP can also be modeled as a continuous process. Games for which the FP procedure eventually leads to players playing equilibrium strategies are said to have the FPP.

Robinson (1951) showed that the discrete - and best known - version of this learning process, converges to a Nash equilibrium ${ }^{4}$. The result that zero-sum games have the FPP when FP is assumed to be a continuous process, is a folk theorem, but is most frequently attributed to Brown (1949). While he never seems to have published a rigorous proof, he makes this claim on several occasions in his work. The result was only formally established by Harris (1998). In this chapter, we obtain an alternative proof, both shorter and easier than the proof of Harris. In particular, it is shown that Brown's claim follows from a result of Monderer, Samet, and Sela (1997).

\footnotetext{
${ }^{4}$ Existence of Nash equilibrium in zero-sum games was established before by von Neumann (1928).
} 



\section{Chapter 2}

\section{Alternating Offers Bargaining with Loss Aversion}

\subsection{Introduction}

One of the characteristics of the Ståhl (1972) and Rubinstein (1982) noncooperative approach to the classical problem of dividing a pie between two players is that preferences are time dependent but do not otherwise depend on the history of play of the game. In real life bargaining situations it is very likely that this assumption is violated and that the share of the pie that an agent finally obtains is evaluated in terms of the history of offers and counter-offers made so far. In particular, it is likely that a share of $x \%$ is evaluated less if a share of $y \%$ with $y>x$ has been within reach at an earlier stage of the game. This is the phenomenon of loss aversion, and the objective in this chapter is to study the effect of loss aversion in the Rubinstein alternating offers bargaining game.

Loss aversion was introduced by Kahneman and Tversky (1979). We adopt the simple and elegant version of Shalev (2002). In this version, an agent's preference is characterized by a basic utility function, a reference point, and a loss aversion coefficient: outcomes below the reference point are regarded as losses and their basic utility values are scaled down by the loss aversion factor. It is essential that this reference point be endogenous (see Tversky and Kahneman 1981). In particular, in a game-theoretic context, what is regarded as a loss is likely to depend on the (history of play of the) 
game.

In this chapter we make the natural assumption that the reference point of a player is equal to the highest turned down offer of the opponent, since that represents the share of the pie that could have been obtained with certainty so far. It turns out that this results in a non-trivial modification of the Rubinstein alternating offers bargaining game: through changed reference points the game with loss aversion depends on the history of play. Indeed, while all subgames in the classical Rubinstein model starting with either a proposal by a player or an acceptance/rejection decision are essentially identical, in our case these subgames depend on the effect the history of play has on preferences. This makes the analysis of the game and, in particular, the characterization of subgame perfect equilibria, much more complicated.

Thus, we consider the Rubinstein alternating offers bargaining game with loss averse players, where the discount factor is interpreted as the probability of continuation of the game after rejection of a proposal. For this game, we construct a subgame perfect equilibrium that shares some features with the subgame perfect equilibrium of the classical game without loss aversion: immediate acceptance of equilibrium offers - implying that agreement is reached immediately as well; indifference between acceptance and rejection of such offers; and strategies depending only on the current reference points. It turns out that the equilibrium strategies depend on nine different regions describing the location of the players' reference points. For instance, if the reference points are high then we are essentially back in the classical case since higher offers are not going to be made in equilibrium and, thus, the reference points will no longer change. But if reference points are low - we assume them to be zero initially - then the equilibrium proposals are influenced by the possibility of future higher reference points. We also show that the constructed equilibrium is the unique subgame perfect equilibrium with the three mentioned properties, but have to leave it as an open problem whether these properties are necessary for uniqueness.

We include an extensive comparative statics analysis of the constructed subgame perfect equilibrium. We establish the intuitive result that higher loss aversion leads to a lower equilibrium share of the pie. We also extend our results to the case of different discount factors (probabilities of continuation of the game), and to more general (increasing and concave) basic utility functions. Finally, we establish an asymptotic relation with non-symmetric Nash bargaining solutions, in the spirit of Binmore et al. (1986). In particular, we show that a player's bargaining power is negatively related to own 
loss aversion and positively to the opponent's loss aversion.

\section{Further related literature}

Loss aversion with a fixed reference point can be regarded as a special case of risk aversion. The effect of risk aversion in the Nash (1950a) and Rubinstein (1982) bargaining models has been studied before, initially in Kihlstrom et al. (1981) and Roth (1985) in the context of expected utility. For the more general context of rank dependent utility, see Safra and Zilcha (1993) and Köbberling and Peters (2003). In all this work the utility functions are fixed and do not change as a result of (play of) the game.

Closer to the present context is Shalev (2002), who applies a loss aversion transformation to the discount factor, and obtains the unique subgame perfect equilibrium of Rubinstein with the transformed discount factors. $\mathrm{Li}$ (2007) assumes that bargainers prefer disagreement over any share that is below the highest they have been offered in the past, and finds a unique subgame perfect equilibrium in this setting. Thus, Li's way of updating reference points during play of the game bears some resemblance with our approach. However, $\mathrm{Li}$ assumes that reference points grow over time. Moreover, the utility functions in Li's model have a discontinuous jump that explicitly ensures that, in equilibrium, each offer made to a player exceeds the previous offer made to that player.

Compte and Jehiel (2003) assume that after breakdown of the negotiations, the game starts anew at a fixed cost. The first mover is randomly chosen from the two bargainers, and in each such new bargaining phase, they have a constant reference point which is based on the proposals made in the previous phases. The subgame perfect equilibrium they find in this setting has in common with Li's that bargainers do not achieve agreement instantaneously.

\section{Organization of the chapter}

Section 2.2 describes the model and in Section 2.3 we construct a subgame perfect equilibrium. Section 2.4 concerns uniqueness of this equilibrium, and Section 2.5 collects our comparative statics results. Section 2.6 concludes. Because of their length, all proofs are collected in three Appendices 2.A.1, 2.A.2, and 2.A.3. 


\subsection{The Alternating Offers Bargaining Model with Loss Aversion}

In this section we describe the alternating offers bargaining model of $\mathrm{Ru}-$ binstein (1982) and introduce the concept of loss aversion in this model. We will try to be as nontechnical as is possible without becoming imprecise. A completely formal treatment is presented in Appendix 2.A.1.

One unit of a perfectly divisible good, the pie, has to be divided among two bargainers, 1 and 2 . The set of all possible partitions of the pie is denoted as

$$
Z:=\left\{\left(z_{1}, z_{2}\right) \in \mathbb{R}^{2} \mid z_{1}+z_{2}=1, z_{1}, z_{2} \geq 0\right\} .
$$

Bargaining takes place at time $t=1,2, \ldots$ At odd moments, player 1 makes a proposal $z=\left(z_{1}, z_{2}\right) \in Z$ and player 2 decides to accept $(Y)$ or to reject $(N)$ this proposal. At even moments, the roles of the players are reversed. If a proposal $\left(z_{1}, z_{2}\right) \in Z$ is accepted, then the game ends and each player $i$ obtains $z_{i}$. If a proposal is rejected then the game continues to the next moment with probability $0<\delta<1$, and stops with probability $1-\delta$. In the latter case, the game ends in disagreement and no player receives anything, i.e., the shares $(0,0)$ result. If the game continues forever - which happens with probability 0 - then again the shares $(0,0)$ result.

A strategy $f$ for player 1 in this game specifies for each odd moment a proposal in $Z$, where this proposal may depend on the complete history of play of the game so far; and for each even moment an answer $Y$ or $N$, where this answer may depend on the current proposal and on the rest of the history of play of the game. A strategy $g$ of player 2 is defined similarly, with the roles of odd and even moments reversed.

So far, this is the Rubinstein alternating offers mechanism. From here, however, we deviate by assuming that the players are loss averse. We assume that the basic utility for a player $i$ of obtaining a share $z_{i}$ of the pie is just equal to $z_{i},{ }^{1}$ but that shares $z_{i}$ below some reference point $r_{i} \in[0,1]$ are regarded as losses and scaled down by a loss aversion coefficient $\lambda_{i} \geq 0$. More precisely, player $i$ evaluates $z_{i}$ by the function

$$
w\left(z_{i}, r_{i}, \lambda_{i}\right):= \begin{cases}z_{i} & \text { if } z_{i} \geq r_{i} \\ z_{i}-\lambda_{i}\left(r_{i}-z_{i}\right) & \text { if } z_{i}<r_{i}\end{cases}
$$

\footnotetext{
${ }^{1}$ This assumption will be relaxed in Section 5 .
} 
or, equivalently, by

$$
w\left(z_{i}, r_{i}, \lambda_{i}\right)=\left(1+\lambda_{i}\right) z_{i}-\lambda_{i} \max \left\{r_{i}, z_{i}\right\}
$$

Loss aversion was first introduced by Kahneman and Tversky (1979). We use the simplified version of Shalev (2002). We assume that the loss aversion coefficients of the bargainers are given and fixed. The reference points of the players, however, are determined endogenously, in the following way. ${ }^{2}$ At some moment $t$, consider all the offers made to player $i$ by the other player $j$ so far, possibly including the offer that is on the table at moment $t$. These represent all the shares of the pie that player $i$ could have obtained up to this moment with certainty. Then, it is natural to assume that the maximum of those shares is player $i$ 's reference point, since this is what he could have obtained: lower shares represent losses with respect to this reference point, and are evaluated according to (2.1).

Thus, we assume that the initial reference points are 0 and write $r_{1}^{0}=$ $r_{2}^{0}=0$; and that at any moment $t \geq 1$, player 1 's and 2 's reference points are equal to

$$
r_{1}^{t}=\max \left\{0, z_{1}^{s} \mid s=2,4, \ldots \leq t\right\}, r_{2}^{t}=\max \left\{z_{2}^{s} \mid s=1,3, \ldots \leq t\right\},
$$

if $z^{1}, z^{2}, \ldots, z^{t} \in Z$ are the proposals made up to time $t$. Observe that incorporating loss aversion in this natural way causes a crucial difference with the classical Rubinstein bargaining game: the game is no longer history independent. For instance, subgames starting at odd moments are no longer identical copies of the whole game, since reference points and therefore payoff functions may have changed during the play of the game.

Recall that the unique subgame perfect equilibrium (SPE) in the classical Rubinstein bargaining game (without loss aversion) has the following characteristics: (i) it is time and history independent, that is, players always make and accept the same proposals; (ii) in equilibrium every proposal is immediately accepted; and (iii) in equilibrium a player is always indifferent between acceptance and rejection. Also in our model with loss aversion we look for an SPE but, clearly, we cannot expect to find a history independent equilibrium. In the next section, we will construct an SPE in which, instead, the strategies of the players are stationary Markov strategies: proposals and

\footnotetext{
${ }^{2}$ Endogeneity of the reference point is an essential assumption in prospect theory, see Tversky and Kahneman (1981). If we would assume exogenous, fixed reference points then our model would reduce to a special case of the standard Rubinstein bargaining model.
} 
acceptance/rejection decisions depend only on the current reference points, and not on time or on the history of play of the game otherwise than through the effect on reference points. This equilibrium will still satisfy (ii) and (iii) and, in fact, we will show that it is the unique SPE with stationary Markov strategies and satisfying (ii) and (iii). Whether dropping one or more of these three conditions allows for different SPE is an open question.

\subsection{A Subgame Perfect Equilibrium}

Heuristically, the SPE in the Rubinstein model is based on the idea that the proposal of a player $i$ should make his opponent $j$ indifferent between that proposal and the proposal of $j$ himself in the next round. We will employ the same idea to construct an SPE in our model with loss averse players.

Consider an odd time moment $t$, where player 1 makes a proposal $x \in Z$. Suppose that after rejection player 2 makes the proposal $y \in Z$ at time $t+1$. Let $r_{2}$ be player 2's reference point at time $t-1$ (so $r_{2}=x_{2}$ at $t=1$ ). To make player 2 accept the proposal $x$ we would need

$$
\begin{aligned}
&\left(1+\lambda_{2}\right) x_{2}-\lambda_{2} \max \left\{r_{2}, x_{2}\right\} \geq \\
& \delta\left[\left(1+\lambda_{2}\right) y_{2}-\lambda_{2} \max \left\{y_{2}, \max \left\{r_{2}, x_{2}\right\}\right\}\right]-(1-\delta) \lambda_{2} \max \left\{r_{2}, x_{2}\right\}
\end{aligned}
$$

That is, player 2 should value the offer $x$ at time $t$ at least as highly as his own offer $y$ in the next period after having rejected $x$. The analogous inequality at even moments is

$$
\begin{aligned}
&\left(1+\lambda_{1}\right) y_{1}-\lambda_{1} \max \left\{r_{1}, y_{1}\right\} \geq \\
& \delta\left[\left(1+\lambda_{1}\right) x_{1}-\lambda_{1} \max \left\{x_{1}, \max \left\{r_{1}, y_{1}\right\}\right\}\right]-(1-\delta) \lambda_{1} \max \left\{r_{1}, y_{1}\right\}
\end{aligned}
$$

To construct the equilibrium, we assume that the inequalities (2.2) and (2.3) are equalities. Let $\mu_{i}:=1+\lambda_{i}(1-\delta)$ for $i=1,2$. Then elaborating (2.3) with equality yields the following three cases:

1. $r_{1}>x_{1}>y_{1}: \delta x_{1}=y_{1}$.

2. $x_{1} \geq r_{1}>y_{1}: \delta x_{1}=\left(1+\lambda_{1}\right) y_{1}-\delta \lambda_{1} r_{1}$.

3. $x_{1}>y_{1} \geq r_{1}: \delta x_{1}=\mu_{1} y_{1}$.

These three cases are exhaustive - it is easy to check that the case $y_{1} \geq x_{1}$ cannot occur. From (2.2) we obtain three similar cases: 
I. $r_{2}>y_{2}>x_{2}: \delta y_{2}=x_{2}$.

II. $y_{2} \geq r_{2}>x_{2}: \delta y_{2}=\left(1+\lambda_{2}\right) x_{2}-\delta \lambda_{2} r_{2}$.

III. $y_{2}>x_{2} \geq r_{2}: \delta y_{2}=\mu_{2} x_{2}$.

By combining these equations we obtain a partition of the unit square $[0,1]^{2}$ of all possible pairs of reference points $\left(r_{1}, r_{2}\right)$ into nine sets. These sets are accurately depicted in Figure 2.1 , and denoted by $X_{1, \mathrm{I}}, \ldots, X_{3, \mathrm{III}}$. Within one and the same set $X$., the equilibrium proposals of the players take the same form, which may or may not depend on the specific values of the reference points within the set.

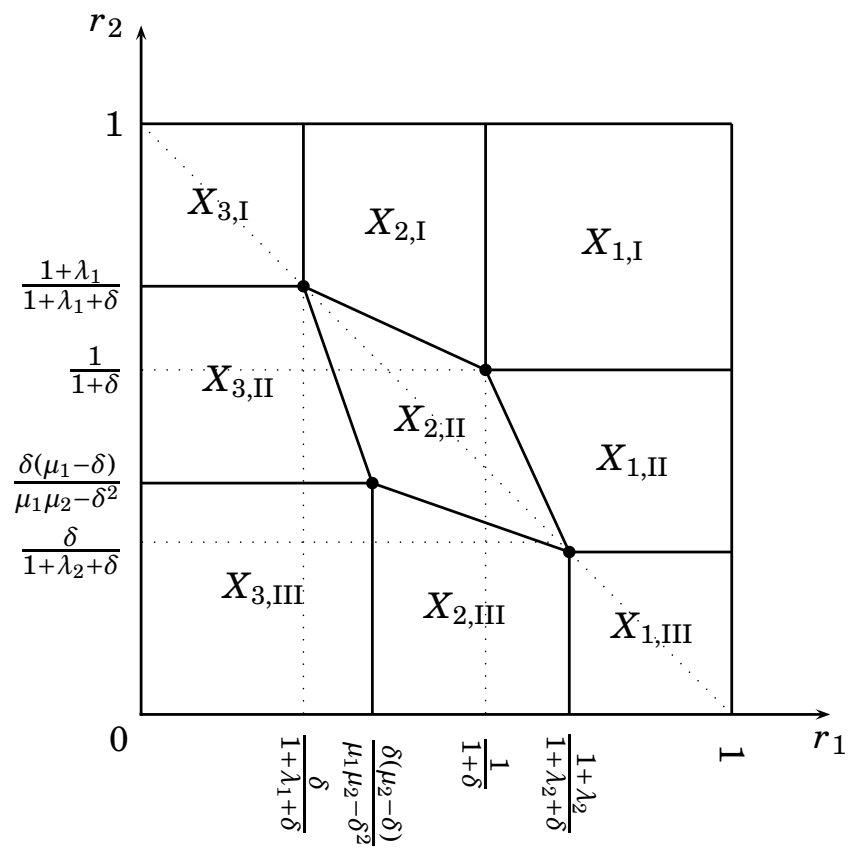

Figure 2.1: The partition $X_{1, \mathrm{I}}, \ldots, X_{3, \mathrm{III}}$.

A formal description of these nine sets and of all associated equilibrium proposals is given in Appendix 2.A.1. Here we limit ourselves to the main aspects.

In the set $X_{1, \mathrm{I}}$ the reference points are relatively high and the classical Rubinstein proposals

$$
x^{1, \mathrm{I}}=\left(\frac{1}{1+\delta}, \frac{\delta}{1+\delta}\right), y^{1, \mathrm{I}}=\left(\frac{\delta}{1+\delta}, \frac{1}{1+\delta}\right) .
$$


obtain. These are independent of the values of the reference points.

Also in the sets $X_{1, \mathrm{III}}$ and $X_{3, \mathrm{I}}$ the equilibrium proposals are constant and do not depend on the reference points. In $X_{3, \mathrm{III}}$, which is the relevant set for the equilibrium outcome in Theorem 2.3.1 below, the equilibrium proposals are

$$
x^{3, \mathrm{III}}=\left(\frac{\mu_{1}\left(\mu_{2}-\delta\right)}{\mu_{1} \mu_{2}-\delta^{2}}, \frac{\delta\left(\mu_{1}-\delta\right)}{\mu_{1} \mu_{2}-\delta^{2}}\right), y^{3, \mathrm{III}}=\left(\frac{\delta\left(\mu_{2}-\delta\right)}{\mu_{1} \mu_{2}-\delta^{2}}, \frac{\mu_{2}\left(\mu_{1}-\delta\right)}{\mu_{1} \mu_{2}-\delta^{2}}\right) .
$$

Again, these proposals do not depend directly on the reference points.

In $X_{1, \mathrm{II}}$ and $X_{3, \mathrm{II}}$ the equilibrium proposals depend explicitly on player 2's reference point but not on player 1's reference point; and in $X_{2, \mathrm{I}}$ and $X_{2, \mathrm{III}}$ the equilibrium proposals depend explicitly on player 1's reference point but not on player 2's reference point. In $X_{2, \text { II }}$ the proposals depend on both players' reference points. Thus, only intermediate valued reference points turn up in the equilibrium proposals explicitly. An equilibrium share is above the reference point if this is low, and below the reference point if this is high.

We now define strategies $\hat{f}$ for player 1 and $\hat{g}$ for player 2 based on the sets $X_{\omega}$ and associated proposals $x^{\omega}, y^{\omega}$, where $\omega \in\{1, \mathrm{I}, \ldots, 3, \mathrm{III}\}$. Consider player 1 . At any odd moment $t$ and for any reference point $\left(r_{1}, r_{2}\right)$, take the (unique) $X_{\omega}$ containing $\left(r_{1}, r_{2}\right)$ : then player 1 makes the corresponding proposal $x^{\omega}$. At any even moment $t$ and for any reference point $\left(r_{1}, r_{2}\right)$, take again the relevant set $X_{\omega}$ : then player 1 accepts a proposal $z$ if and only if $z_{1} \geq y_{1}^{\omega}$. This defines the strategy $\hat{f}$. The strategy $\hat{g}$ for player 2 is defined similarly.

We now have the following result.

Theorem 2.3.1. The strategy profile $(\hat{f}, \hat{g})$ is an SPE. The outcome is

$$
x^{3, \mathrm{III}}=\left(\frac{\mu_{1}\left(\mu_{2}-\delta\right)}{\mu_{1} \mu_{2}-\delta^{2}}, \frac{\delta\left(\mu_{1}-\delta\right)}{\mu_{1} \mu_{2}-\delta^{2}}\right) .
$$

The proof of Theorem 2.3.1 is in Appendix 2.A.1.

\subsection{A Uniqueness Result}

Let $(f, g)$ be a pair of strategies and consider the following possible conditions on $(f, g)$. 
(U1) $f$ and $g$ are stationary Markov strategies. Specifically, the proposal prescribed by $f$ at each odd moment is time independent and depends only on the reference points at that moment, and the $Y / N$ decision prescribed by $f$ at each even moment depends only on player 2's proposal and the reference points at that moment. Similarly for $g$.

(U2) Immediate acceptance. Any proposal made by player 1 according to $f$ is accepted by player 2 according to $g$, and conversely.

(U3) Accept-reject indifference. According to $(f, g)$, player 2 is indifferent between accepting $(\mathrm{Y})$ or rejecting $(\mathrm{N})$ a proposal made by player 1 , and conversely.

Note that these conditions are satisfied by the subgame perfect equilibrium in the classical model (without loss aversion). The equilibrium $(\hat{f}, \hat{g})$ in Theorem 2.3.1 was in fact constructed using these assumptions, which makes the following result intuitive.

Theorem 2.4.1. $(\hat{f}, \hat{g})$ is the unique SPE strategy profile satisfying (U1), (U2), and (U3).

The formal (lengthy) proof is presented in Appendix 2.A.2. We now proceed with a discussion of these conditions.

The condition that the equilibrium strategies are stationary Markov strategies implies that they depend on the history of play of the game only through the effect this play has on the players' reference points. Note that this does not imply that the players are restricted to stationary Markov strategies: the SPE $(\hat{f}, \hat{g})$ is resistent to deviations also with other strategies.

The second condition means that any proposal is immediately accepted. We can in fact show that any SPE must satisfy this condition in some subgames, namely those where the reference points are higher than the (equilibrium) shares of the pie. This is intuitive, cf. the extreme case where the reference points are equal to 1 and, thus, are fixed throughout the rest of the game, so that such a subgame is equivalent to the classical game without loss aversion.

The third condition, finally, requires that each player is indifferent between accepting and rejecting the proposal made by the other player. Note that it follows from the basic equilibrium condition that accepting a proposal must make a player at least as well off as rejecting it. In the classical model with loss neutral players a player $j$ cannot be strictly better off since otherwise the proposing player $i$ could lower the share of $j$ in the proposal and, 
thus, increase his own share and be better off. This argument, however is based on the fact that in the classical model the payoffs of the players do not change as a result of playing the game. In our model we cannot exclude the possibility that such a deviation by the proposing player $i$ may lead to rejection by $j$ since $j$ 's new reference point resulting from the rejection may be lower as it would have been from rejecting the equilibrium proposal. This may effect not only player $j$ 's future payoff but also the play of the game after rejection.

Based on many failed attempts to construct different equilibria we are inclined to think that the conditions (U1)-(U3) are implied by SPE, but, as mentioned, the question is still open.

\subsection{Analysis of the Equilibrium}

In this section we analyze the $\operatorname{SPE}(\hat{f}, \hat{g})$ with respect to the loss aversion coefficients $\lambda_{1}$ and $\lambda_{2}$ and the probability of continuation $\delta$. We consider what happens if $\delta$ goes to 1 and if the players have different $\delta$ 's. We also consider what happens if the time lapse between proposals tends to zero, and establish a relation with asymmetric Nash bargaining solutions.

\subsubsection{Comparative Statics of the Loss Aversion Coefficients}

The result of playing the equilibrium $(\hat{f}, \hat{g})$ is the proposal and immediate acceptance of some distribution of the pie. Here we investigate the dependence of this distribution on the players' loss aversion coefficients. We restrict ourselves to the set of reference point pairs $X_{3, \mathrm{III}}$, since this is the relevant set at the beginning of the game. Moreover, the comparative statics results in subgames where the reference points are in different sets $X_{\omega}$, are similar. Recall that

$$
x^{3, \mathrm{III}}=\left(\frac{\mu_{1}\left(\mu_{2}-\delta\right)}{\mu_{1} \mu_{2}-\delta^{2}}, \frac{\delta\left(\mu_{1}-\delta\right)}{\mu_{1} \mu_{2}-\delta^{2}}\right)
$$

where $\mu_{i}=1+\lambda_{i}(1-\delta)$. It is sufficient to restrict the analysis to one player, because what one player gains is exactly what the other player loses. Differentiating with respect to $\lambda_{1}$ and $\lambda_{2}$ yields

$$
\frac{d x_{1}^{3, \text { III }}}{d \lambda_{1}}=-\frac{\delta^{2}(1-\delta)^{2}\left(1+\lambda_{2}\right)}{\left(\mu_{1} \mu_{2}-\delta^{2}\right)^{2}}<0,
$$


and

$$
\frac{d x_{1}^{3, \mathrm{III}}}{d \lambda_{2}}=\frac{\delta \mu_{1}(1-\delta)^{2}\left(1+\lambda_{1}\right)}{\left(\mu_{1} \mu_{2}-\delta^{2}\right)^{2}}>0 .
$$

Thus, players are hurt by their own loss aversion and benefit from their opponent's. This result also holds for the other subgames. However, if a player's reference point is very high, i.e., $r_{1} \geq x_{1}^{\omega}$ where $\omega \in\{1, \mathrm{I}, 1, \mathrm{II}, 1, \mathrm{III}\}$ or $r_{2} \geq y_{2}^{\omega}$ where $\omega \in\{3, \mathrm{I}, 2, \mathrm{I}, 1, \mathrm{I}\}$, then the equilibrium is independent of this player's degree of loss aversion. For instance, if $\left(r_{1}, r_{2}\right) \in X_{1, \mathrm{II}}$, then $\lambda_{1}$ has no influence on the equilibrium partition, but $\lambda_{2}$ has a positive effect on player 1 's payoff and a negative effect on player 2's.

\subsubsection{Convergence Results with respect to the Probability of Continuation}

\section{Convergence of the SPE for a common $\delta$}

The question we consider here is what happens to the equilibrium if $\delta$ tends to one. Using l'Hôpital's rule we derive

$$
\lim _{\delta \rightarrow 1} x_{1}^{3, \text { III }}=\frac{1+\lambda_{2}}{2+\lambda_{1}+\lambda_{2}}
$$

and hence

$$
\lim _{\delta \rightarrow 1} x_{2}^{3, \mathrm{III}}=\frac{1+\lambda_{1}}{2+\lambda_{1}+\lambda_{2}} .
$$

Observe that player $i$ 's payoff is conversely proportional to $1+\lambda_{j}$, where $i, j \in\{1,2\}$ and $i \neq j$.

We can repeat this for all subgames. Figure 2.2 shows the nine sets of Figure 2.1 in the limit for $\delta$ going to 1 and the limit equilibrium proposals, for the case where $\lambda_{2}>\lambda_{1}$. The limit outcome in $X_{1, \mathrm{I}}$ is an equal split of the pie, $(1 / 2,1 / 2)$. In $X_{3, \mathrm{II}}$ and $X_{1, \mathrm{II}}$ the limit equilibrium partition is $\left(1-r_{2}, r_{2}\right)$, while in $X_{2, \mathrm{III}}$ and $X_{2, \mathrm{I}}$ it is $\left(r_{1}, 1-r_{1}\right)$. In $X_{3, \mathrm{I}}$ the limit equilibrium partition is $\left(1 /\left(2+\lambda_{1}\right),\left(1+\lambda_{1}\right) /\left(2+\lambda_{1}\right)\right)$, and in $X_{1, \mathrm{III}}$ it is $\left(\left(1+\lambda_{2}\right) /\left(2+\lambda_{2}\right), 1 /\left(2+\lambda_{2}\right)\right)$. Set $X_{2, \mathrm{II}}$ collapses to the line piece $r_{1}+r_{2}=1$ where $r_{1}$ is in the interval $\left(1 /\left(2+\lambda_{1}\right),\left(1+\lambda_{2}\right) /\left(2+\lambda_{2}\right)\right)$. The limit equilibrium partition associated with this set is $\left(r_{1}, r_{2}\right)$. 


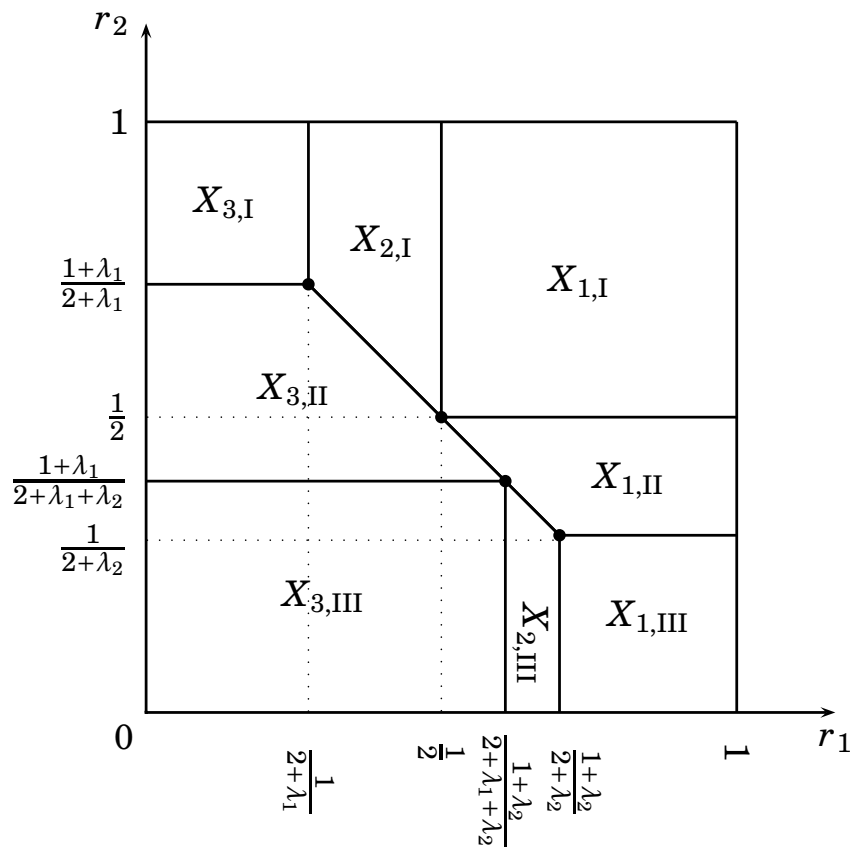

Figure 2.2: The equilibrium partitions for $\delta \rightarrow 1$, with $\lambda_{2}>\lambda_{1}$.

\section{Convergence of the SPE for $\delta_{1} \neq \delta_{2}$}

We generalize the model to the situation where the players have individual continuation probabilities $\delta_{1}$ and $\delta_{2}$. In our setting, $\delta_{i}$ is interpreted as the probability that the game continues after player $i$ rejected $j$ 's proposal. Inequalities (2.2) and (2.3) generalize to

$$
\begin{aligned}
& \left(1+\lambda_{2}\right) x_{2}-\lambda_{2} \max \left\{r_{2}, x_{2}\right\} \geq \\
& \delta_{2}\left(\left(1+\lambda_{2}\right) y_{2}-\lambda_{2} \max \left\{y_{2}, \max \left\{r_{2}, x_{2}\right\}\right\}\right)-\left(1-\delta_{2}\right) \lambda_{2} \max \left\{r_{2}, x_{2}\right\}
\end{aligned}
$$

and

$$
\begin{aligned}
& \left(1+\lambda_{1}\right) y_{1}-\lambda_{1} \max \left\{r_{1}, y_{1}\right\} \geq \\
& \delta_{1}\left(\left(1+\lambda_{2}\right) y_{2}-\lambda_{2} \max \left\{y_{2}, \max \left\{r_{2}, x_{2}\right\}\right\}\right)-\left(1-\delta_{1}\right) \lambda_{2} \max \left\{r_{2}, x_{2}\right\} .
\end{aligned}
$$

All prior results also apply to this more general model. In particular, we obtain a solution to the above inequalities, considered as equalities, from 
which we construct a strategy profile. This strategy profile is the unique SPE satisfying (U1)-(U3).

A further generalization that leaves the previous results intact, is when there is a time lapse $\Delta$ between proposals, and the waiting time for breakdown of the game after player $i, i=1,2$, rejected the last proposal, is exponentially distributed with survival rate $\beta_{i}$. Then the probability that the game continues after player $i$ rejected a proposal is $\delta_{i}^{\Delta}$, where $\delta_{i}=$ $\exp \left(-1 / \beta_{i}\right)$. Since the game starts with the reference points in $X_{3, \mathrm{III}}$, we limit the analysis to this case. The SPE outcome is

$$
\begin{aligned}
& x^{3, \mathrm{III}}=\left(\frac{\mu_{1}\left(\mu_{2}-\delta_{2}^{\Delta}\right)}{\mu_{1} \mu_{2}-\delta_{1}^{\Delta} \delta_{2}^{\Delta}}, \frac{\delta_{2}^{\Delta}\left(\mu_{1}-\delta_{1}^{\Delta}\right)}{\mu_{1} \mu_{2}-\delta_{1}^{\Delta} \delta_{2}^{\Delta}}\right) \\
& y^{3, \mathrm{III}}=\left(\frac{\delta_{1}^{\Delta}\left(\mu_{2}-\delta_{2}^{\Delta}\right)}{\mu_{1} \mu_{2}-\delta_{1}^{\Delta} \delta_{2}^{\Delta}}, \frac{\mu_{2}\left(\mu_{1}-\delta_{1}^{\Delta}\right)}{\mu_{1} \mu_{2}-\delta_{1}^{\Delta} \delta_{2}^{\Delta}}\right)
\end{aligned}
$$

where now $\mu_{i}:=1+\lambda_{i}\left(1-\delta_{i}^{\Delta}\right)$ for $i=1,2$. We can derive

$$
\lim _{\Delta \rightarrow 0} x^{3, \mathrm{III}}=\left(\frac{\left(1+\lambda_{2}\right) \log \delta_{2}}{\left(1+\lambda_{1}\right) \log \delta_{1}+\left(1+\lambda_{2}\right) \log \delta_{2}}, \frac{\left(1+\lambda_{1}\right) \log \delta_{1}}{\left(1+\lambda_{1}\right) \log \delta_{1}+\left(1+\lambda_{2}\right) \log \delta_{2}}\right) .
$$

In a similar way we obtain

$$
\lim _{\Delta \rightarrow 0} y^{3, \mathrm{III}}=\lim _{\Delta \rightarrow 0} x^{3, \mathrm{III}} .
$$

Note that this is an asymmetric Nash bargaining solution as described by Harsanyi and Selten (1972) and Kalai (1977). That is, it is the solution to the optimization problem

$$
\arg \max _{z \in Z} z_{1}^{\alpha} z_{2}^{1-\alpha}
$$

where

$$
\alpha=\frac{\left(1+\lambda_{2}\right) \log \delta_{2}}{\left(1+\lambda_{1}\right) \log \delta_{1}+\left(1+\lambda_{2}\right) \log \delta_{2}} .
$$

In Appendix 2.A.3, we extend this result to the situation where players have concave utility functions $v_{i}:[0,1] \rightarrow[0,1]$, and the feasible set associated with the partitions of the pie is

$$
\tilde{Z}:=\left\{\left(v_{1}(\gamma), v_{2}(1-\gamma)\right) \mid 0 \leq \gamma \leq 1\right\} .
$$


We construct an SPE, resulting in an $x \in \tilde{Z}$, which is a function of $\delta_{1}^{\Delta}$ and $\delta_{2}^{\Delta}$. Using an argument from Binmore et al. (1986) we show that if $\Delta$ goes to zero, then the SPE outcome $x$ converges to $\tilde{z}^{N}$ where

$$
\tilde{z}^{N}:=\underset{z \in \tilde{Z}}{\arg \max } z_{1}^{\alpha} z_{2}^{1-\alpha},
$$

with $\alpha$ as defined by (2.4). Thus, increased loss aversion of a player results in increased 'bargaining power' of the opponent.

\subsection{Concluding Remarks}

In this paper we have investigated the effect of loss aversion in the strategic bargaining game of alternating offers introduced by Rubinstein (1982), by constructing a subgame perfect equilibrium in the extended model, and performing a comparative statics analysis on the outcome with respect to the bargainers' loss aversion coefficients. We find that being loss averse has a negative effect on a player's equilibrium share. We further find that the outcome of the bargaining procedure converges to an asymmetric Nash bargaining solution if the exogenous probability of breakdown goes to zero, such that higher loss aversion leads to higher bargaining power of the opponent.

This subgame perfect equilibrium is the unique SPE that shares three distinguishing features - stationary Markov strategies, immediate acceptance, and accept-reject indifference - with the unique SPE of Rubinstein. It is an open question whether uniqueness also holds without all or some of these conditions.

\section{A Appendix: Proofs}

\section{A.1 The Formal Model and the SPE}

In this section we formally complete the description of the model and show that the constructed strategy profile $(\hat{f}, \hat{g})$ is an SPE (i.e., we prove Theorem 2.3.1).

\section{Formal Model}

We divide $T=\mathbb{N}$ into $T_{\text {odd }}:=\{1,3, \ldots\}$ and $T_{\text {even }}:=\{2,4, \ldots\}$. We assume that players have full information about the history of play: at any time $t \in T$, 
they know all previous proposals, their own as well as those of the other player.

Define $h^{t}$, the history of the game at time $t \in T$, as a vector of players' proposals which have taken place before and at time $t$. Specifically, $h^{t}:=$ $\left(z^{1}, \ldots, z^{t}\right)$, where $z^{s} \in Z$ for all $s \leq t$. Furthermore, define $H^{t}$ as the set of all possible histories $h^{t}$ of the bargaining procedure at time $t \in T$. That is, $H^{t}:=\prod_{s=1}^{t} Z$. Furthermore, let $H^{0}:=\left\{h^{0}\right\}$, where $h^{0}$ is the empty history. Henceforth, the term 'history' is used to indicate non-empty histories.

The players' strategies are elements in $F$ or $G$ where $F$ is the set of infinite sequences of functions $\left(f^{t}\right)_{t \in T}$ where

$$
\begin{array}{ll}
\text { for } t=1: & f^{t} \in Z, \\
\text { for } t>1 \text { and } t \in T_{\text {odd }}: & f^{t}: H^{t-1} \rightarrow Z, \\
\text { for } t \in T_{\text {even }}: & f^{t}: H^{t} \rightarrow\{\mathrm{Y}, \mathrm{N}\},
\end{array}
$$

and $G$ the set of infinite sequences of functions $\left(g^{t}\right)_{t \in T}$ where

$$
\begin{array}{ll}
\text { for } t \in T_{\text {odd }}: & g^{t}: H^{t} \rightarrow\{\mathrm{Y}, \mathrm{N}\} \\
\text { for } t \in T_{\text {even }}: & g^{t}: H^{t-1} \rightarrow Z
\end{array}
$$

An agreement path $\left(h^{t}, a\right)$ is a history $h^{t} \in H^{t}$ ending in acceptance of the time $t$ proposal. The set

$$
A^{t}:=\left\{\left(h^{t}, a\right) \mid h^{t} \in H^{t}\right\}
$$

contains all time $t$ agreement paths. The set

$$
A:=\bigcup_{t \in T} A^{t}
$$

contains all histories ending in agreement. Similarly, we define a disagreement path $\left(h^{t}, d\right)$ as a history $h^{t} \in H^{t}$ ending in breakdown of the negotiations upon rejection of the time $t$ proposal. We define $D^{t}:=\left\{\left(h^{t}, d\right) \mid h^{t} \in H^{t}\right\}$ and $D:=\bigcup_{t \in T} D^{t}$. The set $C^{t}$ contains all objects of the form $\left(h^{t}, c\right)$, i.e. histories that do not end at time $t$. Finally, we define

$$
H^{\infty}:=\left\{\left(z^{1}, z^{2}, \ldots\right) \mid z^{t} \in Z \text { for all } t \in T\right\},
$$


and refer to elements of $H^{\infty}$ as infinite paths. Then

$$
\bar{H}:=H^{\infty} \cup A \cup D
$$

is the set containing all paths of the game. Observe that a strategy profile $(f, g) \in F \times G$ determines a specific play of the game or, equivalently, a set of paths in $\bar{H}$. In particular, if $(f, g)$ leads to agreement on a partition at time $t$, then the set of paths associated with that strategy profile contains $t-1$ paths in $D$ and one in $A$. If $(f, g)$ never induces an agreement, then this set contains a single path in $H^{\infty}$, and countably many in $D$.

We introduce the function $\xi_{i}: \bar{H} \backslash H^{\infty} \rightarrow[0,1]$ that specifies for each finite path in $\bar{H}$ the (physical) share of the pie bargainer $i$ obtains. Specifically, for all $h^{t} \in H, h^{t}=\left(z^{1}, \ldots, z^{t}\right)$, we have

$$
\begin{aligned}
& \xi_{i}\left(h^{t}, a\right):=z_{i}^{t}, \text { and } \\
& \xi_{i}\left(h^{t}, d\right):=0 .
\end{aligned}
$$

We define player i's utility function for (dis)agreement paths as

$$
u_{i}\left(h^{t}, a\right):=w\left(\xi_{i}\left(h^{t}, a\right), r_{i}\left(h^{t}\right), \lambda_{i}\right), u_{i}\left(h^{t}, d\right):=w\left(\xi_{i}\left(h^{t}, d\right), r_{i}\left(h^{t}\right), \lambda_{i}\right) .
$$

Furthermore, player $i$ 's utility evaluation of paths in $H^{\infty}$, i.e., perpetual disagreement, is defined as $-\lambda_{i}$. That is,

$$
u_{i}(h):=-\lambda_{i} \text { for all } h \in H^{\infty} .
$$

Finally, we define the expected utility function $U_{i}: F \times G \rightarrow \mathbb{R}$. Let $t \in T$ be the point in time up until which the history is known, and let players play the strategy profile $(f, g) \in F \times G$ from then on. Then we say they play $\left(f\left|h^{t}, g\right| h^{t}\right)$ at time $t+1$, and we denote by $U_{i}\left(f\left|h^{t}, g\right| h^{t}\right)$ player $i$ 's time $t$ expected utility from the strategy profile $(f, g) \in F \times G$. This can be exactly calculated, and is known to both players.

The strategy profile $(f, g)$ is a subgame perfect equilibrium (SPE) if for every $t \in T$ and every $h^{t} \in H^{t}$, we have

$$
\begin{aligned}
& U_{1}\left(f\left|h^{t}, g\right| h^{t}\right) \geq U_{1}\left(\tilde{f}\left|h^{t}, g\right| h^{t}\right) \text { for all } \tilde{f}, \text { and } \\
& U_{2}\left(f\left|h^{t}, g\right| h^{t}\right) \geq U_{2}\left(f\left|h^{t}, \tilde{g}\right| h^{t}\right) \text { for all } \tilde{g} .
\end{aligned}
$$

\section{The Strategy Pair $(\hat{\mathbf{f}}, \hat{\mathbf{g}})$}

We first formally describe the nine sets, also referred to as regions, and associated proposals on which the definition of $(\hat{f}, \hat{g})$ is based. 
Definition of $X_{\omega}$ and $x^{\omega}, y^{\omega}$

\section{- Region 1,I}

$$
X_{1, \mathrm{I}}=\left\{\left(r_{1}, r_{2}\right) \in[0,1]^{2} \mid r_{1}>\frac{1}{1+\delta}, r_{2}>\frac{1}{1+\delta}\right\} .
$$

The equilibrium proposals in $X_{1, \mathrm{I}}$ are

$$
x^{1, \mathrm{I}}=\left(\frac{1}{1+\delta}, \frac{\delta}{1+\delta}\right), y^{1, \mathrm{I}}=\left(\frac{\delta}{1+\delta}, \frac{1}{1+\delta}\right) .
$$

[Thus, if both players' reference points lie above $1 /(1+\delta)$, then the regular Rubinstein outcomes are obtained. In Figure 2.1, the Rubinstein outcome is the South-West corner point of this set.]

\section{- Region 1,III}

$$
X_{1, \mathrm{III}}=\left\{\left(r_{1}, r_{2}\right) \in[0,1]^{2} \mid r_{1}>\frac{1+\lambda_{2}}{1+\lambda_{2}+\delta}, r_{2} \leq \frac{\delta}{1+\lambda_{2}+\delta}\right\} .
$$

The equilibrium proposals in $X_{1, \mathrm{III}}$ are

$$
\begin{aligned}
& x^{1, \mathrm{III}}=\left(\frac{1+\lambda_{2}}{1+\lambda_{2}+\delta}, \frac{\delta}{1+\lambda_{2}+\delta}\right) \\
& y^{1, \mathrm{III}}=\left(\frac{\delta\left(1+\lambda_{2}\right)}{1+\lambda_{2}+\delta}, \frac{1+\lambda_{2}(1-\delta)}{1+\lambda_{2}+\delta}\right) .
\end{aligned}
$$

[Observe that this set is a square, and that its North-West corner point lies on the line $r_{1}+r_{2}=1$.]

\section{- Region 3,I}

$$
X_{3, \mathrm{I}}=\left\{\left(r_{1}, r_{2}\right) \in[0,1]^{2} \mid r_{1} \leq \frac{\delta}{1+\lambda_{1}+\delta}, r_{2}>\frac{1+\lambda_{1}}{1+\lambda_{1}+\delta}\right\} .
$$

This set is similar to $X_{1, \mathrm{III}}$, but with the roles of the players reversed. The equilibrium proposals are

$$
\begin{aligned}
& x^{3, \mathrm{I}}=\left(\frac{1+\lambda_{1}(1-\delta)}{1+\lambda_{1}+\delta}, \frac{\delta\left(1+\lambda_{1}\right)}{1+\lambda_{1}+\delta}\right) \\
& y^{3, \mathrm{I}}=\left(\frac{\delta}{1+\lambda_{1}+\delta}, \frac{1+\lambda_{1}}{1+\lambda_{1}+\delta}\right) .
\end{aligned}
$$

[This set is again a square, and its South-East corner point lies on the line $r_{1}+r_{2}=1$.] 


\section{- Region 3,III}

$$
X_{3, \mathrm{III}}=\left\{\left(r_{1}, r_{2}\right) \in[0,1]^{2} \mid r_{1} \leq \frac{\delta\left(\mu_{2}-\delta\right)}{\mu_{1} \mu_{2}-\delta^{2}}, r_{2} \leq \frac{\delta\left(\mu_{1}-\delta\right)}{\mu_{1} \mu_{2}-\delta^{2}}\right\} .
$$

The equilibrium proposals are

$$
x^{3, \mathrm{III}}=\left(\frac{\mu_{1}\left(\mu_{2}-\delta\right)}{\mu_{1} \mu_{2}-\delta^{2}}, \frac{\delta\left(\mu_{1}-\delta\right)}{\mu_{1} \mu_{2}-\delta^{2}}\right), y^{3, \mathrm{III}}=\left(\frac{\delta\left(\mu_{2}-\delta\right)}{\mu_{1} \mu_{2}-\delta^{2}}, \frac{\mu_{2}\left(\mu_{1}-\delta\right)}{\mu_{1} \mu_{2}-\delta^{2}}\right) .
$$

\section{- Region 1,II}

$$
X_{1, \mathrm{II}}=\left\{\left(r_{1}, r_{2}\right) \in[0,1]^{2} \mid r_{1}>\frac{\left(\mu_{2}-\delta\right)+\delta \lambda_{2}\left(1-r_{2}\right)}{\left(1+\lambda_{2}\right)-\delta^{2}}, \frac{\delta}{1+\lambda_{2}+\delta}<r_{2} \leq \frac{1}{1+\delta}\right\} .
$$

The equilibrium proposals are

$$
\begin{aligned}
& x^{1, \mathrm{II}}=\left(\frac{\left(\mu_{2}-\delta\right)+\delta \lambda_{2}\left(1-r_{2}\right)}{\left(1+\lambda_{2}\right)-\delta^{2}}, \frac{\left.\delta(1-\delta)+\delta \lambda_{2} r_{2}\right)}{\left(1+\lambda_{2}\right)-\delta^{2}}\right) \\
& y^{1, \mathrm{II}}=\left(\frac{\delta\left(\mu_{2}-\delta\right)+\delta^{2} \lambda_{2}\left(1-r_{2}\right)}{\left(1+\lambda_{2}\right)-\delta^{2}}, \frac{\left(\mu_{2}-\delta\right)+\delta^{2} \lambda_{2} r_{2}}{\left(1+\lambda_{2}\right)-\delta^{2}}\right) .
\end{aligned}
$$

[Observe that these proposals now depend on player 2's reference point.]

\section{- Region 3,II}

$$
\begin{aligned}
& X_{3, \mathrm{II}}=\left\{\left(r_{1}, r_{2}\right) \in[0,1]^{2} \mid r_{1} \leq \frac{\delta\left(\mu_{2}-\delta+\delta \lambda_{2}\left(1-r_{2}\right)\right)}{\mu_{1}\left(1+\lambda_{2}\right)-\delta^{2}},\right. \\
& \left.\frac{\delta\left(\mu_{1}-\delta\right)}{\mu_{1} \mu_{2}-\delta^{2}}<r_{2} \leq \frac{1+\lambda_{1}}{1+\lambda_{1}+\delta}\right\} .
\end{aligned}
$$

The equilibrium proposals are

$$
\begin{aligned}
& x^{3, \mathrm{II}}=\left(\frac{\mu_{1}\left(\mu_{2}-\delta+\lambda_{2} \delta\left(1-r_{2}\right)\right)}{\mu_{1}\left(1+\lambda_{2}\right)-\delta^{2}}, \frac{\delta\left(\mu_{1}-\delta+\lambda_{2} r_{2} \mu_{1}\right)}{\mu_{1}\left(1+\lambda_{2}\right)-\delta^{2}}\right) \\
& y^{3, \mathrm{II}}=\left(\frac{\delta\left(\mu_{2}-\delta+\delta \lambda_{2}\left(1-r_{2}\right)\right)}{\mu_{1}\left(1+\lambda_{2}\right)-\delta^{2}}, \frac{\left(\mu_{1}-\delta\right)\left(1+\lambda_{2}\right)+\delta^{2} \lambda_{2} r_{2}}{\mu_{1}\left(1+\lambda_{2}\right)-\delta^{2}}\right) .
\end{aligned}
$$

[In this set player 1's reference point is low, which explains that the equilibrium proposals do not depend on it.]

\section{- Region 2,I}

$$
X_{2, \mathrm{I}}=\left\{\left(r_{1}, r_{2}\right) \in[0,1]^{2} \mid \frac{\delta}{1+\lambda_{1}+\delta}<r_{1} \leq \frac{1}{1+\delta}, r_{2}>\frac{\left(\mu_{1}-\delta\right)+\delta \lambda_{1}\left(1-r_{1}\right)}{\left(1+\lambda_{1}\right)-\delta^{2}}\right\} \text {. }
$$


The equilibrium proposals are

$$
\begin{aligned}
& x^{2 . \mathrm{I}}=\left(\frac{\left(\mu_{1}-\delta\right)+\delta^{2} \lambda_{1} r_{1}}{\left(1+\lambda_{1}\right)-\delta^{2}}, \frac{\delta\left(\mu_{1}-\delta\right)+\delta^{2} \lambda_{1}\left(1-r_{1}\right)}{\left(1+\lambda_{1}\right)-\delta^{2}}\right) \\
& y^{2, \mathrm{I}}=\left(\frac{\left.\delta(1-\delta)+\delta \lambda_{1} r_{1}\right)}{\left(1+\lambda_{1}\right)-\delta^{2}}, \frac{\left(\mu_{1}-\delta\right)+\delta \lambda_{1}\left(1-r_{1}\right)}{\left(1+\lambda_{1}\right)-\delta^{2}}\right) .
\end{aligned}
$$

[This set is similar to $X_{1, \mathrm{II}}$.]

\section{- Region 2,III}

$$
\begin{aligned}
& X_{2, \mathrm{III}}=\left\{\left(r_{1}, r_{2}\right) \in[0,1]^{2} \mid \frac{\delta\left(\mu_{2}-\delta\right)}{\mu_{1} \mu_{2}-\delta^{2}} \leq r_{1} \leq \frac{1+\lambda_{2}}{1+\lambda_{2}+\delta},\right. \\
& \left.r_{2} \leq \frac{\delta\left(\left(\mu_{1}-\delta\right)+\delta \lambda_{1}\left(1-r_{1}\right)\right)}{\left(1+\lambda_{1}\right) \mu_{2}-\delta^{2}}\right\} .
\end{aligned}
$$

The equilibrium proposals are

$$
\begin{aligned}
& x^{2, \mathrm{III}}=\left(\frac{\left(1+\lambda_{1}\right)\left(\mu_{2}-\delta\right)+\delta^{2} \lambda_{1} r_{1}}{\left(1+\lambda_{1}\right) \mu_{2}-\delta^{2}}, \frac{\delta\left(\left(\mu_{1}-\delta\right)+\delta \lambda_{1}\left(1-r_{1}\right)\right)}{\left(1+\lambda_{1}\right) \mu_{2}-\delta^{2}}\right) \\
& y^{2, \mathrm{III}}=\left(\frac{\delta\left(\left(\mu_{2}-\delta\right)+\mu_{2} \lambda_{1} r_{1}\right)}{\left(1+\lambda_{1}\right) \mu_{2}-\delta^{2}}, \frac{\mu_{2}\left(\left(\mu_{1}-\delta\right)+\delta \lambda_{1}\left(1-r_{1}\right)\right)}{\left(1+\lambda_{1}\right) \mu_{2}-\delta^{2}}\right) .
\end{aligned}
$$

[In this set player 2's reference point is low, which explains that the equilibrium proposals do not depend on it.]

\section{- Region 2,II}

The boundaries of $X_{2, \text { II }}$ are described by the boundaries of the neighboring sets, see Figure 2.1. The equilibrium proposals $x^{2, \mathrm{II}}$ and $y^{2, \mathrm{II}}$ are given by

$$
\begin{aligned}
& x_{1}^{2, \mathrm{II}}=\frac{\left(1+\lambda_{1}\right)\left(\left(\mu_{2}-\delta\right)+\delta \lambda_{2}\left(1-r_{2}\right)\right)+\delta^{2} \lambda_{1} r_{1}}{\left(1+\lambda_{1}\right)\left(1+\lambda_{2}\right)-\delta^{2}}, \\
& x_{2}^{2, \mathrm{II}}=\frac{\delta\left(\mu_{1}-\delta+\delta \lambda_{1}\left(1-r_{1}\right)+\lambda_{2}\left(1+\lambda_{1}\right) r_{2}\right)}{\left(1+\lambda_{1}\right)\left(1+\lambda_{2}\right)-\delta^{2}}, \\
& y_{1}^{2, \mathrm{II}}=\frac{\delta\left(\mu_{2}-\delta+\delta \lambda_{2}\left(1-r_{2}\right)+\lambda_{1}\left(1+\lambda_{2}\right) r_{1}\right)}{\left(1+\lambda_{1}\right)\left(1+\lambda_{2}\right)-\delta^{2}}, \\
& y_{2}^{2, \mathrm{II}}=\frac{\left(1+\lambda_{2}\right)\left(\left(\mu_{1}-\delta\right)+\delta \lambda_{1}\left(1-r_{1}\right)\right)+\delta^{2} \lambda_{2} r_{2}}{\left(1+\lambda_{1}\right)\left(1+\lambda_{2}\right)-\delta^{2}} .
\end{aligned}
$$




\section{The Proof of Theorem 2.3.1}

To prove Theorem 2.3.1, we make use of the one-deviation property. The one-deviation property says that for a strategy profile $(f, g) \in F \times G$ to be a subgame perfect equilibrium it is sufficient that no player can improve by deviating only once, i.e., at one point in time.

Hendon, Jacobsen, and Sloth (1996) showed that the one-deviation property holds in infinite-horizon extensive-form games which are continuous at infinity $^{3}$. Continuity at infinity is defined as follows. For any $\varepsilon>0$ there is a number $t \in T$ such that if two strategy profiles $(f, g),\left(f^{\prime}, g^{\prime}\right) \in F \times G$ are such that $\left(f^{s}, g^{s}\right)=\left(f^{\prime s}, g^{\prime s}\right)$ for all $s \leq t$, then $\left|U_{i}(f, g)-U_{i}\left(f^{\prime}, g^{\prime}\right)\right|<\varepsilon$.

Lemma 2.A.1. The bargaining game with loss averse players is continuous at infinity.

Proof. Let $\varepsilon>0$, and let $(f, g)$ and $\left(f^{\prime}, g^{\prime}\right)$ be strategy profiles in $F \times G$ satisfying $\left(f^{s}, g^{s}\right)=\left(f^{\prime s}, g^{\prime s}\right)$ for all $s \leq t$, where $t>\max _{i=1,2} \log _{\delta} \varepsilon /\left(1+\lambda_{i}\right)$. Observe that the largest payoff difference between two such strategy profiles would emerge when the one yields player $i$ the whole pie at time $t+1$, while the other leads to perpetual disagreement. In the former case, player $i$ would obtain

$$
\bar{U}_{i}=\delta^{t}+(1-\delta) \sum_{s=1}^{t} \delta^{s-1} u_{i}\left(h^{s}, d\right),
$$

while in the latter he would obtain

$$
\underline{U}_{i}=(1-\delta) \sum_{s=1}^{\infty} \delta^{s-1} u_{i}\left(h^{s}, d\right) .
$$

From the fact that $u_{i}\left(h^{t}, d\right) \geq-\lambda_{i}$ for all $t \in T$, it follows that

$$
\begin{aligned}
\underline{U}_{i} & =(1-\delta) \sum_{s=1}^{t} \delta^{s-1} u_{i}\left(h^{s}, d\right)+(1-\delta) \sum_{s=t+1}^{\infty} \delta^{s-1} u_{i}\left(h^{s}, d\right) \\
& \geq(1-\delta) \sum_{s=1}^{t} \delta^{s-1} u_{i}\left(h^{s}, d\right)+\delta^{t}(1-\delta)\left(-\lambda_{i}\right) \frac{1}{1-\delta} \\
& =-\delta^{t} \lambda_{i}+(1-\delta) \sum_{s=1}^{t} \delta^{s-1} u_{i}\left(h^{s}, d\right) .
\end{aligned}
$$

\footnotetext{
${ }^{3}$ Another requirement concerns Bayesian updating, but this is automatically fulfilled since our game is one of perfect information.
} 
From this and the fact that $\bar{U}_{i}-\underline{U}_{i} \geq 0$, we obtain

$$
\begin{array}{r}
\left|U_{i}(f, g)-U_{i}\left(f^{\prime}, g^{\prime}\right)\right| \leq \bar{U}_{i}-\underline{U}_{i} \leq \delta^{t}+(1-\delta) \sum_{s=1}^{t} \delta^{s-1} u_{i}\left(h^{s}, d\right) \\
+\delta^{t} \lambda_{i}-(1-\delta) \sum_{s=1}^{t} \delta^{s-1} u_{i}\left(h^{s}, d\right)=\delta^{t}\left(1+\lambda_{i}\right)<\varepsilon .
\end{array}
$$

Hence, the game is continuous at infinity.

It follows from Lemma 2.A.1 that we can use the one-deviation property.

Denote $\Omega=\{1$. I. , .., 3.III. $\}$.

Proof of Theorem 2.3.1. To show that $(\hat{f}, \hat{g})$ is SPE, it is sufficient to show that there is no subgame in which player 1 (2) can profitably deviate from $\hat{f}$ $(\hat{g})$ at a single time $t \in T$, given that player 2 (1) plays strategy $\hat{g}(\hat{f})$. Assume player 2 plays strategy $\hat{g}$. We denote the utility player 1 obtains by following strategy $\hat{f}$ by $u_{1}^{*}$.

Let $h^{t-1} \in C^{t-1}$, i.e. $h^{t-1}$ is a history continuing to the next period. Assume $h^{t-1}$ is such that $\left(r_{1}\left(h^{t-1}\right), r_{2}\left(h^{t-1}\right)\right) \in X_{\omega}$ with $\omega \in \Omega$, and that $h^{t}=$ $\left(h^{t-1}, z\right)$ with $z \in Z$. If $t$ is odd (even), then $z$ is proposed by player 1 (2). If the proposal $z$ is rejected, then the game continues with probability $\delta$ to the next period or ends with probability $1-\delta$. If the game continues to time $t+1$, then it ends in immediate acceptance of the proposal at $t+1$, since $(\hat{f}, \hat{g})$ is the prevalent strategy profile.

To show that $\hat{f}$ is a best reply to $\hat{g}$ we distinguish two cases, namely $t$ is odd and $t$ is even. For each case, we consider three subcases. The reference point pair $\left(r_{1}, r_{2}\right)$ is in $X_{\omega}$ where

Case 1. $\omega \in\{1, \mathrm{I}, 1, \mathrm{II}, 1, \mathrm{III}\}$. Then $r_{1}>x_{1}^{\omega}>y_{1}^{\omega}$, and $y_{1}^{\omega}=\delta x_{1}^{\omega}$.

Case 2. $\omega \in\{2, \mathrm{I}, 2, \mathrm{II}, 2, \mathrm{III}\}$. Then $x_{1}^{\omega} \geq r_{1}>y_{1}^{\omega}$, and $y_{1}^{\omega}=\frac{\delta\left(x_{1}^{\omega}+\lambda_{1} r_{1}\right)}{1+\lambda_{1}}$.

Case 3. $\omega \in\{3, \mathrm{I}, 3$,II, 3,III $\}$. Then $x_{1}^{\omega}>y_{1}^{\omega} \geq r_{1}$, and $y_{1}^{\omega}=\frac{\delta}{\mu_{1}} x_{1}^{\omega}$.

$t$ odd, case 1. We distinguish between the following cases:

- $z_{1}=x_{1}^{\omega}$ : In this case player 1 follows strategy $\hat{f}$. Player 2 accepts, so

$$
u_{1}^{*}=u_{1}\left(h^{t}, a\right)=\left(1+\lambda_{1}\right) x_{1}^{\omega}-\lambda_{1} \max \left\{r_{1}, x_{1}^{\omega}\right\}=\left(1+\lambda_{1}\right) x_{1}^{\omega}-\lambda_{1} r_{1} .
$$


- $z_{1}<x_{1}^{\omega}$ : Then $z_{2}>x_{2}^{\omega}$, so player 2 accepts. Player 1's payoff is $u_{1}\left(h^{t}, a\right)=$ $\left(1+\lambda_{1}\right) z_{1}-\lambda_{1} \max \left\{r_{1}, z_{1}\right\}$. Observe that $r_{1} \geq x_{1}^{\omega}>z_{1}$ implies that

$$
u_{1}\left(h^{t}, a\right)=\left(1+\lambda_{1}\right) z_{1}-\lambda_{1} r_{1} .
$$

Then $z_{1}<x_{1}^{\omega}$ implies $u_{1}\left(h^{t}, a\right)<u_{1}^{*}$. Hence, the proposal $z$ is not optimal.

- $z_{1}>x_{1}^{\omega}$ : Then $z_{2}<x_{2}^{\omega}$, so player 2 rejects and proposes $y^{\omega}$ if the game continues. Hence, player 1 obtains

$$
\begin{aligned}
& \delta u_{1}\left(h^{t+1}, a\right)+(1-\delta) u_{1}\left(h^{t}, d\right) \\
= & \delta\left(\left(1+\lambda_{1}\right) y_{1}^{\omega}-\lambda_{1} \max \left\{y_{1}^{\omega}, r_{1}\right\}\right)-(1-\delta) \lambda_{1} r_{1} \\
= & \left(1+\lambda_{1}\right) \delta y_{1}^{\omega}-\delta \lambda_{1} r_{1}-(1-\delta) \lambda_{1} r_{1} \\
= & \left(1+\lambda_{1}\right) \delta y_{1}^{\omega}-\lambda_{1} r_{1} .
\end{aligned}
$$

Since $x_{1}^{\omega}>\delta^{2} x_{1}^{\omega}=\delta y_{1}^{\omega}$, we have $\delta u_{1}\left(h^{t+1}, a\right)+(1-\delta) u_{1}\left(h^{t}, d\right)<u_{1}^{*}$. Thus, the proposal $z$ is not optimal.

$t$ odd, case 2. We distinguish between three cases:

- $z_{1}=x_{1}^{\omega}$ : In this case player 1 follows strategy $\hat{f}$. Player 2 accepts, so

$$
u_{1}^{*}=u_{1}\left(h^{t}, a\right)=\left(1+\lambda_{1}\right) x_{1}^{\omega}-\lambda_{1} \max \left\{x_{1}^{\omega}, r_{1}\right\}=x_{1}^{\omega} .
$$

- $z_{1}<x_{1}^{\omega}$ : Since then $z_{2}>x_{2}^{\omega}$, player 2 accepts. Player 1's payoff is $u_{1}\left(h^{t}, a\right)=$ $\left(1+\lambda_{1}\right) z_{1}-\lambda_{1} \max \left\{r_{1}, z_{1}\right\}$. From

$$
\left(1+\lambda_{1}\right) z_{1}-\lambda_{1} \max \left\{r_{1}, z_{1}\right\} \leq z_{1}<x_{1}^{\omega}=u_{1}^{*},
$$

it follows that the proposal $z$ is not optimal.

- $z_{1}>x_{1}^{\omega}$ : Since then $z_{2}<x_{2}^{\omega}$, player 2 rejects, and proposes $y^{\omega}$ if the game continues. Hence, player 1 obtains

$$
\begin{aligned}
& \delta u_{1}\left(h^{t+1}, a\right)+(1-\delta) u_{1}\left(h^{t}, d\right) \\
= & \left(1+\lambda_{1}\right) \delta y_{1}^{\omega}-\delta \lambda_{1} \max \left\{y_{1}^{\omega}, r_{1}\right\}-(1-\delta) \lambda_{1} r_{1} \\
= & \left(1+\lambda_{1}\right) \delta y_{1}^{\omega}-\lambda_{1} r_{1} .
\end{aligned}
$$

From $y_{1}^{\omega}=\frac{\delta\left(x_{1}^{\omega}+\lambda_{1} r_{1}\right)}{1+\lambda_{1}}$ it follows that $\delta x_{1}^{\omega}=\left(1+\lambda_{1}\right) y_{1}^{\omega}-\delta \lambda_{1} r_{1}$. Therefore,

$$
u_{1}^{*}=x_{1}^{\omega}=\left(1+\lambda_{1}\right) \frac{1}{\delta} y_{1}^{\omega}-\lambda_{1} r_{1}>\left(1+\lambda_{1}\right) \delta y_{1}^{\omega}-\lambda_{1} r_{1} .
$$


Thus, the proposal $z$ is not optimal.

$t$ odd, case 3. We distinguish between three cases:

- $z_{1}=x_{1}^{\omega}$ : In this case player 1 follows strategy $\hat{f}$. Player 2 accepts, so

$$
u_{1}^{*}=u_{1}\left(h^{t}, a\right)=\left(1+\lambda_{1}\right) x_{1}^{\omega}-\lambda_{1} \max \left\{x_{1}^{\omega}, r_{1}\right\}=x_{1}^{\omega} .
$$

- $z_{1}<x_{1}^{\omega}$ : Since then $z_{2}>x_{2}^{\omega}$, player 2 accepts. Player 1's payoff is $u_{1}\left(h^{t}, a\right)=$ $\left(1+\lambda_{1}\right) z_{1}-\lambda_{1} \max \left\{r_{1}, z_{1}\right\}$. From

$$
\left(1+\lambda_{1}\right) z_{1}-\lambda_{1} \max \left\{r_{1}, z_{1}\right\} \leq z_{1}<x_{1}^{\omega},
$$

it follows that $u_{1}^{*}>u_{1}\left(h^{t}, a\right)$. Thus, the proposal $z$ is not optimal.

- $z_{1}>x_{1}^{\omega}$ : Since $z_{2}<x_{2}^{\omega}$, player 2 rejects, and proposes $y^{\omega}$ if the game continues. Hence, player 1 obtains

$$
\begin{aligned}
& \delta u_{1}\left(h^{t+1}, a\right)+(1-\delta) u_{1}\left(h^{t}, d\right) \\
= & \left(1+\lambda_{1}\right) \delta y_{1}^{\omega}-\delta \lambda_{1} \max \left\{y_{1}^{\omega}, r_{1}\right\}-(1-\delta) \lambda_{1} r_{1} \\
= & \delta y_{1}^{\omega}-(1-\delta) \lambda_{1} r_{1} .
\end{aligned}
$$

Observe that

$$
u_{1}^{*}=x_{1}^{\omega}>y_{1}^{\omega}>\delta y_{1}^{\omega} \geq \delta y_{1}^{\omega}-(1-\delta) \lambda_{1} r_{1} .
$$

Hence, the proposal $z$ is not optimal.

$t$ even, case 1. Assume player 2 proposes some $z \in Z$. Accepting gives player 1

$$
u_{1}\left(h^{t}, a\right)=\left(1+\lambda_{1}\right) z_{1}-\lambda_{1} \max \left\{r_{1}, z_{1}\right\}
$$

Rejecting makes his reference point switch to $\max \left\{r_{1}, z_{1}\right\}$; if the game continues, he proposes $x^{\omega}$, and player 2 accepts. Since $r_{1}>x_{1}^{\omega}$ and $y_{1}^{\omega}=\delta x_{1}^{\omega}$, we have

$$
\begin{aligned}
& \delta u_{1}\left(h^{t+1}, a\right)+(1-\delta) u_{1}\left(h^{t}, d\right) \\
= & \left(1+\lambda_{1}\right) \delta x_{1}^{\omega}-\delta \lambda_{1} \max \left\{x_{1}^{\omega}, \max \left\{r_{1}, z_{1}\right\}\right\}-(1-\delta) \lambda_{1} \max \left\{r_{1}, z_{1}\right\} \\
= & \left(1+\lambda_{1}\right) \delta x_{1}^{\omega}-\delta \lambda_{1} \max \left\{r_{1}, z_{1}\right\}-(1-\delta) \lambda_{1} \max \left\{r_{1}, z_{1}\right\} \\
= & \left(1+\lambda_{1}\right) y_{1}^{\omega}-\lambda_{1} \max \left\{r_{1}, z_{1}\right\} .
\end{aligned}
$$


Hence, it is optimal to accept the proposal $z$ if $z_{1} \geq y_{1}^{\omega}$, and to reject it otherwise. That is, to play strategy $\hat{f}$.

$t$ even, case 2. Assume player 2 proposes some $z \in Z$ with $z_{1} \leq x_{1}^{\omega^{\prime}}$ where $\omega^{\prime} \in\{1, \mathrm{I}, 1, \mathrm{II}, 1, \mathrm{IIII}\}$. Accepting gives player 1

$$
u_{1}\left(h^{t}, a\right)=\left(1+\lambda_{1}\right) z_{1}-\lambda_{1} \max \left\{r_{1}, z_{1}\right\}
$$

If he rejects and the game continues, then the reference point $r_{1}\left(h^{t}\right)$ is $\max \left\{r_{1}, z_{1}\right\}$. Thus, at $t+1$ we enter a new game in which $\left(\max \left\{r_{1}, z_{1}\right\}, r_{2}\right)$ is the prevalent reference point pair. Since $\left(\max \left\{r_{1}, z_{1}\right\}, r_{2}\right) \in \omega$ where $\omega \in$ $\{2, \mathrm{I}, 2$,II, 2 ,III $\}$, we have $x_{1}^{\omega} \geq \max \left\{r_{1}, z_{1}\right\} \geq y_{1}^{\omega}$, where $y_{1}^{\omega}$ is equal to $\delta\left(x_{1}^{\omega}+\right.$ $\left.\lambda_{1} \max \left\{r_{1}, z_{1}\right\}\right) /\left(1+\lambda_{1}\right)$. At $t+1$ player 1 proposes $x_{1}^{\omega}$ and player 2 accepts, so rejecting $z$ yields

$$
\begin{aligned}
& \delta u_{1}\left(h^{t+1}, a\right)+(1-\delta) u_{1}\left(h^{t}, d\right) \\
= & \left(1+\lambda_{1}\right) \delta x_{1}^{\omega}-\delta \lambda_{1} \max \left\{x_{1}^{\omega}, \max \left\{r_{1}, z_{1}\right\}\right\}-(1-\delta) \lambda_{1} \max \left\{r_{1}, z_{1}\right\} \\
= & \delta x_{1}^{\omega}-(1-\delta) \lambda_{1} \max \left\{r_{1}, z_{1}\right\} \\
= & \left(1+\lambda_{1}\right) y_{1}^{\omega}-\lambda_{1} \max \left\{r_{1}, z_{1}\right\} .
\end{aligned}
$$

If player 2 proposes $z \in Z$ with $z_{1}>x_{1}^{\omega^{\prime}}$, then accepting yields $u_{1}\left(h^{t}, a\right)=z_{1}$ and rejecting

$$
\begin{aligned}
& \delta u_{1}\left(h^{t+1}, a\right)+(1-\delta) u_{1}\left(h^{t}, d\right) \\
= & \left(1+\lambda_{1}\right) \delta x_{1}^{\omega^{\prime}}-\delta \lambda_{1} \max \left\{x_{1}^{\omega^{\prime}}, \max \left\{r_{1}, z_{1}\right\}\right\}-(1-\delta) \lambda_{1} \max \left\{r_{1}, z_{1}\right\} \\
= & \left(1+\lambda_{1}\right) y_{1}^{\omega^{\prime}}-\lambda_{1} z_{1} .
\end{aligned}
$$

Since $z_{1}>x_{1}^{\omega^{\prime}}>y_{1}^{\omega^{\prime}}$, we have $u_{1}\left(h^{t}, a\right)>\delta u_{1}\left(h^{t+1}, a\right)+(1-\delta) u_{1}\left(h^{t}, d\right)$. In general, it is optimal to accept the proposal $z$ if $z_{1} \geq y_{1}^{\omega}$, and to reject it otherwise. That is, it is optimal to follow strategy $\hat{f}$.

$t$ even, case 3. Assume player 2 proposes some $z \in Z$ with $z_{1}<y_{1}^{\omega}$. Note that $r_{1}<x_{1}^{\omega}$. Hence, rejecting yields

$$
\begin{aligned}
& \delta u_{1}\left(h^{t+1}, a\right)+(1-\delta) u_{1}\left(h^{t}, d\right) \\
= & \left(1+\lambda_{1}\right) \delta x_{1}^{\omega}-\delta \lambda_{1} \max \left\{x_{1}^{\omega}, \max \left\{r_{1}, z_{1}\right\}\right\}-(1-\delta) \lambda_{1} \max \left\{r_{1}, z_{1}\right\} \\
= & \delta x_{1}^{\omega}-(1-\delta) \lambda_{1} \max \left\{r_{1}, z_{1}\right\} .
\end{aligned}
$$


Accepting yields $u_{1}\left(h^{t}, a\right)=\left(1+\lambda_{1}\right) z_{1}-\lambda_{1} \max \left\{r_{1}, z_{1}\right\}$. Since $y_{1}^{\omega}=\frac{\delta}{\mu_{1}} x_{1}^{\omega}$, we have

$$
\begin{aligned}
u_{1}\left(h^{t}, a\right) & =\left(1+\lambda_{1}\right) z_{1}-\lambda_{1} \max \left\{r_{1}, z_{1}\right\} \\
& =\left(1+\lambda_{1}\right) z_{1}-\delta \lambda_{1} \max \left\{r_{1}, z_{1}\right\}-(1-\delta) \lambda_{1} \max \left\{r_{1}, z_{1}\right\} \\
& <\left(1+\lambda_{1}\right) y_{1}^{\omega}-\delta \lambda_{1} \max \left\{r_{1}, y_{1}^{\omega}\right\}-(1-\delta) \lambda_{1} \max \left\{r_{1}, z_{1}\right\} \\
& =\left(1+\lambda_{1}\right) y_{1}^{\omega}-\delta \lambda_{1} y_{1}^{\omega}-(1-\delta) \lambda_{1} \max \left\{r_{1}, z_{1}\right\} \\
& =\mu_{1} y_{1}^{\omega}-(1-\delta) \lambda_{1} \max \left\{r_{1}, z_{1}\right\} \\
& =\delta x_{1}^{\omega}-(1-\delta) \lambda_{1} \max \left\{r_{1}, z_{1}\right\} .
\end{aligned}
$$

Thus, it is optimal to reject $z$. If player 2 proposes $z \in Z$ with $z_{1} \geq y_{1}^{\omega}$, then accepting yields

$$
u_{1}\left(h^{t}, a\right)=\left(1+\lambda_{1}\right) z_{1}-\lambda_{1} \max \left\{r_{1}, z_{1}\right\}=z_{1} .
$$

Let $z_{1} \leq y_{1}^{\omega^{\prime}}$ where $\omega^{\prime} \in\{1, \mathrm{I}, 1, \mathrm{II}, 1, \mathrm{III}\}$. If player 1 rejects and the game continues, then $r_{1}\left(h^{t}\right)=z_{1}$, and $\left(z_{1}, r_{2}\right) \in \omega^{\prime \prime}$ where $\omega^{\prime \prime} \in\{2, \mathrm{I}, 2, \mathrm{II}, 2$,III $\}$. Note that then $y_{1}^{\omega^{\prime \prime}} \leq z_{1} \leq x_{1}^{\omega^{\prime \prime}}$. Thus, rejecting yields

$$
\begin{aligned}
& \delta u_{1}\left(h^{t+1}, a\right)+(1-\delta) u_{1}\left(h^{t}, d\right) \\
= & \left(1+\lambda_{1}\right) \delta x_{1}^{\omega^{\prime \prime}}-\delta \lambda_{1} \max \left\{x_{1}^{\omega^{\prime \prime}}, z_{1}\right\}-(1-\delta) \lambda_{1} z_{1} \\
= & \delta x_{1}^{\omega^{\prime \prime}}-(1-\delta) \lambda_{1} z_{1} \\
= & \left(1+\lambda_{1}\right) y_{1}^{\omega^{\prime \prime}}-\lambda_{1} z_{1} .
\end{aligned}
$$

Since $z_{1} \geq y_{1}^{\omega^{\prime \prime}}$, also $z_{1} \geq\left(1+\lambda_{1}\right) y_{1}^{\omega^{\prime \prime}}-\lambda_{1} z_{1}$. If player 2 proposes $z \in Z$ with $z_{1}>x_{1}^{\omega^{\prime}}$, then rejecting yields

$$
\begin{aligned}
& \delta u_{1}\left(h^{t+1}, a\right)+(1-\delta) u_{1}\left(h^{t}, d\right) \\
= & \left(1+\lambda_{1}\right) \delta x_{1}^{\omega^{\prime}}-\delta \lambda_{1} \max \left\{x_{1}^{\omega^{\prime}}, \max \left\{r_{1}, z_{1}\right\}\right\}-(1-\delta) \lambda_{1} \max \left\{r_{1}, z_{1}\right\} \\
= & \left(1+\lambda_{1}\right) \delta x_{1}^{\omega^{\prime}}-\delta \lambda_{1} z_{1}-(1-\delta) \lambda_{1} z_{1} \\
= & \left(1+\lambda_{1}\right) \delta x_{1}^{\omega^{\prime}}-\lambda_{1} z_{1} .
\end{aligned}
$$

Observe that $z_{1}>x_{1}^{\omega^{\prime}}$ implies $z_{1}>\delta x_{1}^{\omega^{\prime}}$. It follows that $z_{1}>\left(1+\lambda_{1}\right) \delta x_{1}^{\omega^{\prime}}-\lambda_{1} z_{1}$, i.e. accepting $z$ is optimal.

Thus, we have shown that player 1 can not profitably deviate from $\hat{f}$ at any single time $t$, given that player 2 plays strategy $\hat{g}$. The proof that player 2 can not profitably deviate from $\hat{g}$ at any single time $t$, given that player 1 plays $\hat{f}$, is analogous. Lemma 2.A.1 implies that $(\hat{f}, \hat{g})$ is an SPE. 


\section{A.2 The Proof of Theorem 2.4.1}

Throughout this section, we assume that conditions (U1)-(U3) are satisfied.

\section{Preliminary lemmas}

Define a bargaining round as an offer made by player 1 and a counter offer made by player 2 . Bargaining rounds are indexed with $i \in\{0,1,2, \ldots\}$. Then $\left(r_{1}^{i}, r_{2}^{i}\right)$ is the reference point pair prevalent at the beginning of bargaining round $i$. In view of (U1) it makes sense to define

$$
r_{1}^{i+1}:=\max \left\{r_{1}^{i}, y_{1}\left(r_{1}^{i}, r_{2}^{i+1}\right)\right\}, \text { and } r_{2}^{i+1}:=\max \left\{r_{2}^{i}, x_{2}\left(r_{1}^{i}, r_{2}^{i}\right)\right\} .
$$

This allows us to show the following.

Lemma 2.A.2. Given that $x\left(r_{1}^{i}, r_{2}^{i}\right)$ is player 1's SPE proposal and $y\left(r_{1}^{i}, r_{2}^{i+1}\right)$ player 2's counterproposal, we have

$$
x_{2}\left(r_{1}^{i}, r_{2}^{i}\right)= \begin{cases}\delta-\delta y_{1}\left(r_{1}^{i}, r_{2}^{i+1}\right) & \text { if } r_{2}^{i}>y_{2}\left(r_{1}^{i}, r_{2}^{i+1}\right)>x_{2}\left(r_{1}^{i}, r_{2}^{i}\right) \\ \frac{\delta\left(1+\lambda_{2} r_{2}^{i+1}\right)}{1+\lambda_{2}}-\frac{\delta y_{1}\left(r_{1}^{i}, r_{2}^{i+1}\right)}{1+\lambda_{2}} & \text { if } y_{2}\left(r_{1}^{i}, r_{2}^{i+1}\right) \geq r_{2}^{i}>x_{2}\left(r_{1}^{i}, r_{2}^{i}\right) \\ \frac{\delta}{\mu_{2}}-\frac{\delta}{\mu_{2}} y_{1}\left(r_{1}^{i}, r_{2}^{i+1}\right) & \text { if } y_{2}\left(r_{1}^{i}, r_{2}^{i+1}\right)>x_{2}\left(r_{1}^{i}, r_{2}^{i}\right) \geq r_{2}^{i},\end{cases}
$$

and

$$
y_{1}\left(r_{1}^{i}, r_{2}^{i+1}\right)= \begin{cases}\delta-\delta x_{2}\left(r_{1}^{i+1}, r_{2}^{i+1}\right) & \text { if } r_{1}^{i}>x_{1}\left(r_{1}^{i+1}, r_{2}^{i+1}\right)>y_{1}\left(r_{1}^{i}, r_{2}^{i+1}\right) \\ \frac{\delta\left(1+\lambda_{1} r_{1}^{i+1}\right)}{1+\lambda_{1}}-\frac{\delta x_{2}\left(r_{1}^{i+1}, r_{2}^{i+1}\right)}{1+\lambda_{1}} & \text { if } x_{1}\left(r_{1}^{i+1}, r_{2}^{i+1}\right) \geq r_{1}^{i}>y_{1}\left(r_{1}^{i}, r_{2}^{i+1}\right) \\ \frac{\delta}{\mu_{1}}-\frac{\delta}{\mu_{1}} x_{2}\left(r_{1}^{i+1}, r_{2}^{i+1}\right) & \text { if } x_{1}\left(r_{1}^{i+1}, r_{2}^{i+1}\right)>y_{1}\left(r_{1}^{i}, r_{2}^{i+1}\right) \geq r_{1}^{i} .\end{cases}
$$

Proof. This follows from (U2) and (U3), and the definition of the players' utility functions.

For each $\omega \in \Omega$ we introduce the following sets $P_{\omega}$ and $Q_{\omega}$ of pairs of reference points:

$$
\begin{aligned}
& P_{1, \mathrm{I}}:=\left\{\left(r_{1}^{i}, r_{2}^{i}\right) \mid r_{2}^{i}>y_{2}\left(r_{1}^{i}, r_{2}^{i+1}\right), r_{1}^{i}>x_{1}\left(r_{1}^{i+1}, r_{2}^{i+1}\right)\right\} \\
& P_{2, \mathrm{I}}:=\left\{\left(r_{1}^{i}, r_{2}^{i}\right) \mid r_{2}^{i}>y_{2}\left(r_{1}^{i}, r_{2}^{i+1}\right), x_{1}\left(r_{1}^{i+1}, r_{2}^{i+1}\right) \geq r_{1}^{i}>y_{1}\left(r_{1}^{i}, r_{2}^{i+1}\right)\right\}
\end{aligned}
$$




$$
\begin{aligned}
& P_{3, \mathrm{I}}:=\left\{\left(r_{1}^{i}, r_{2}^{i}\right) \mid r_{2}^{i}>y_{2}\left(r_{1}^{i}, r_{2}^{i+1}\right), y_{1}\left(r_{1}^{i}, r_{2}^{i+1}\right) \geq r_{1}^{i}\right\} \\
& P_{1, \mathrm{II}}:=\left\{\left(r_{1}^{i}, r_{2}^{i}\right) \mid y_{2}\left(r_{1}^{i}, r_{2}^{i+1}\right) \geq r_{2}^{i}>x_{2}\left(r_{1}^{i}, r_{2}^{i}\right), r_{1}^{i}>x_{1}\left(r_{1}^{i+1}, r_{2}^{i+1}\right)\right\} \\
& P_{2, \mathrm{II}}:=\left\{\left(r_{1}^{i}, r_{2}^{i}\right) \mid y_{2}\left(r_{1}^{i}, r_{2}^{i+1}\right) \geq r_{2}^{i}>x_{2}\left(r_{1}^{i}, r_{2}^{i}\right), x_{1}\left(r_{1}^{i+1}, r_{2}^{i+1}\right) \geq r_{1}^{i}>y_{1}\left(r_{1}^{i}, r_{2}^{i+1}\right)\right\} \\
& P_{3, \mathrm{II}}:=\left\{\left(r_{1}^{i}, r_{2}^{i}\right) \mid y_{2}\left(r_{1}^{i}, r_{2}^{i+1}\right) \geq r_{2}^{i}>x_{2}\left(r_{1}^{i}, r_{2}^{i}\right), y_{1}\left(r_{1}^{i}, r_{2}^{i+1}\right) \geq r_{1}^{i}\right\} \\
& P_{1, \mathrm{III}}:=\left\{\left(r_{1}^{i}, r_{2}^{i}\right) \mid x_{2}\left(r_{1}^{i}, r_{2}^{i}\right) \geq r_{2}^{i}, r_{1}^{i}>x_{1}\left(r_{1}^{i+1}, r_{2}^{i+1}\right)\right\} \\
& P_{2, \mathrm{III}}:=\left\{\left(r_{1}^{i}, r_{2}^{i}\right) \mid x_{2}\left(r_{1}^{i}, r_{2}^{i}\right) \geq r_{2}^{i}, x_{1}\left(r_{1}^{i+1}, r_{2}^{i+1}\right) \geq r_{1}^{i}>y_{1}\left(r_{1}^{i}, r_{2}^{i+1}\right)\right\} \\
& P_{3, \mathrm{III}}:=\left\{\left(r_{1}^{i}, r_{2}^{i}\right) \mid x_{2}\left(r_{1}^{i}, r_{2}^{i}\right) \geq r_{2}^{i}, y_{1}\left(r_{1}^{i}, r_{2}^{i+1}\right) \geq r_{1}^{i}\right\} \\
& Q_{1, \mathrm{I}}:=\left\{\left(r_{1}^{i}, r_{2}^{i+1}\right) \mid r_{1}^{i}>x_{1}\left(r_{1}^{i+1}, r_{2}^{i+1}\right), r_{2}^{i+1}>y_{2}\left(r_{1}^{i+1}, r_{2}^{i+2}\right)\right\} \\
& Q_{1, \mathrm{II}}:=\left\{\left(r_{1}^{i}, r_{2}^{i+1}\right) \mid r_{1}^{i}>x_{1}\left(r_{1}^{i+1}, r_{2}^{i+1}\right), y_{2}\left(r_{1}^{i+1}, r_{2}^{i+2}\right) \geq r_{2}^{i+1}>x_{2}\left(r_{1}^{i+1}, r_{2}^{i+1}\right)\right\} \\
& Q_{1, \mathrm{III}}:=\left\{\left(r_{1}^{i}, r_{2}^{i+1}\right) \mid r_{1}^{i}>x_{1}\left(r_{1}^{i+1}, r_{2}^{i+1}\right), x_{2}\left(r_{1}^{i+1}, r_{2}^{i+1}\right) \geq r_{2}^{i+1}\right\} \\
& Q_{2, \mathrm{I}}:=\left\{\left(r_{1}^{i}, r_{2}^{i+1}\right) \mid x_{1}\left(r_{1}^{i+1}, r_{2}^{i+1}\right) \geq r_{1}^{i}>y_{1}\left(r_{1}^{i}, r_{2}^{i+1}\right), r_{2}^{i+1}>y_{2}\left(r_{1}^{i+1}, r_{2}^{i+2}\right)\right\} \\
& Q_{2, \mathrm{II}}:=\left\{\left(r_{1}^{i}, r_{2}^{i+1}\right) \mid x_{1}\left(r_{1}^{i+1}, r_{2}^{i+1}\right) \geq r_{1}^{i}>y_{1}\left(r_{1}^{i}, r_{2}^{i+1}\right), y_{2}\left(r_{1}^{i+1}, r_{2}^{i+2}\right) \geq r_{2}^{i+1}>\right. \\
& \left.x_{2}\left(r_{1}^{i+1}, r_{2}^{i+1}\right)\right\} \\
& Q_{2, \mathrm{III}}:=\left\{\left(r_{1}^{i}, r_{2}^{i+1}\right) \mid x_{1}\left(r_{1}^{i+1}, r_{2}^{i+1}\right) \geq r_{1}^{i}>y_{1}\left(r_{1}^{i}, r_{2}^{i+1}\right), x_{2}\left(r_{1}^{i+1}, r_{2}^{i+1}\right) \geq r_{2}^{i+1}\right\} \\
& Q_{3, \mathrm{I}}:=\left\{\left(r_{1}^{i}, r_{2}^{i+1}\right) \mid y_{1}\left(r_{1}^{i}, r_{2}^{i+1}\right) \geq r_{1}^{i}, r_{2}^{i+1}>y_{2}\left(r_{1}^{i+1}, r_{2}^{i+2}\right)\right\} \\
& Q_{3, \mathrm{II}}:=\left\{\left(r_{1}^{i}, r_{2}^{i+1}\right) \mid y_{1}\left(r_{1}^{i}, r_{2}^{i+1}\right) \geq r_{1}^{i}, y_{2}\left(r_{1}^{i+1}, r_{2}^{i+2}\right) \geq r_{2}^{i+1}>x_{2}\left(r_{1}^{i+1}, r_{2}^{i+1}\right)\right\} \\
& Q_{3, \mathrm{III}}:=\left\{\left(r_{1}^{i}, r_{2}^{i+1}\right) \mid y_{1}\left(r_{1}^{i}, r_{2}^{i+1}\right) \geq r_{1}^{i}, x_{2}\left(r_{1}^{i+1}, r_{2}^{i+1}\right) \geq r_{2}^{i+1}\right\} \text {. }
\end{aligned}
$$

We derive a series of lemmas for reference point pairs in these sets.

Lemma 2.A.3. For all $\left(r_{1}^{i}, r_{2}^{i}\right) \in P_{\omega}$, we have

$$
x_{2}\left(r_{1}^{i}, r_{2}^{i}\right) \geq x_{2}\left(r_{1}^{i+1}, r_{2}^{i+1}\right) \Leftrightarrow x_{2}\left(r_{1}^{i}, r_{2}^{i}\right) \leq x_{2}^{\omega} .
$$


Similarly, for all $\left(r_{1}^{i}, r_{2}^{i+1}\right) \in Q_{\omega}$, we have

$$
y_{1}\left(r_{1}^{i}, r_{2}^{i+1}\right) \geq y_{1}\left(r_{1}^{i+1}, r_{2}^{i+2}\right) \Leftrightarrow y_{1}\left(r_{1}^{i}, r_{2}^{i+1}\right) \leq y_{1}^{\omega} .
$$

Proof. Let $\omega=1$, I and $\left(r_{1}^{i}, r_{2}^{i}\right) \in P_{\omega}$. By definition of $P_{1, \mathrm{I}}$ we have

$$
r_{2}^{i}>y_{2}\left(r_{1}^{i}, r_{2}^{i+1}\right) \text { and } r_{1}^{i}>x_{1}\left(r_{1}^{i+1}, r_{2}^{i+1}\right) .
$$

From Lemma 2.A.2 we obtain

$$
\begin{aligned}
x_{2}\left(r_{1}^{i}, r_{2}^{i}\right) & =\delta-\delta y_{1}\left(r_{1}^{i}, r_{2}^{i+1}\right) \\
& =\delta-\delta\left(\delta-\delta x_{2}\left(r_{1}^{i+1}, r_{2}^{i+1}\right)\right) \\
& =\delta-\delta^{2}+\delta^{2} x_{2}\left(r_{1}^{i+1}, r_{2}^{i+1}\right) .
\end{aligned}
$$

Suppose $x_{2}\left(r_{1}^{i+1}, r_{2}^{i+1}\right) \geq x_{2}\left(r_{1}^{i}, r_{2}^{i}\right)$. Then by (2.10)

$$
x_{2}\left(r_{1}^{i}, r_{2}^{i}\right)=\delta-\delta^{2}+\delta^{2} x_{2}\left(r_{1}^{i+1}, r_{2}^{i+1}\right) \geq \delta-\delta^{2}+\delta^{2} x_{2}\left(r_{1}^{i}, r_{2}^{i}\right) .
$$

It follows that

$$
x_{2}\left(r_{1}^{i}, r_{2}^{i}\right) \geq \frac{\delta-\delta^{2}}{1-\delta^{2}}=\frac{\delta}{1+\delta}=x_{2}^{1, \mathrm{I}} .
$$

Suppose $x_{2}\left(r_{1}^{i+1}, r_{2}^{i+1}\right)<x_{2}\left(r_{1}^{i}, r_{2}^{i}\right)$. Then by (2.10)

$$
x_{2}\left(r_{1}^{i}, r_{2}^{i}\right)=\delta-\delta^{2}+\delta^{2} x_{2}\left(r_{1}^{i+1}, r_{2}^{i+1}\right)<\delta-\delta^{2}+\delta^{2} x_{2}\left(r_{1}^{i}, r_{2}^{i}\right),
$$

implying $x_{2}\left(r_{1}^{i}, r_{2}^{i}\right)<x_{2}^{1, \mathrm{I}}$. Therefore,

$$
x_{2}\left(r_{1}^{i}, r_{2}^{i}\right) \geq x_{2}\left(r_{1}^{i+1}, r_{2}^{i+1}\right) \Leftrightarrow x_{2}\left(r_{1}^{i}, r_{2}^{i}\right) \leq x_{2}^{1, \mathrm{I}} .
$$

Similarly, let $\omega=1, \mathrm{I}$ and $\left(r_{1}^{i}, r_{2}^{i+1}\right) \in Q_{\omega}$. By definition of $Q_{1, \mathrm{I}}$

$$
r_{1}^{i}>x_{1}\left(r_{1}^{i+1}, r_{2}^{i+1}\right) \text { and } r_{2}^{i+1}>y_{2}\left(r_{1}^{i+1}, r_{2}^{i+2}\right) .
$$

Then by Lemma 2.A.2

$$
\begin{aligned}
y_{1}\left(r_{1}^{i}, r_{2}^{i+1}\right) & =\delta-\delta x_{2}\left(r_{1}^{i+1}, r_{2}^{i+1}\right) \\
& =\delta-\delta\left(\delta-\delta y_{1}\left(r_{1}^{i+1}, r_{2}^{i+2}\right)\right) \\
& =\delta-\delta^{2}+\delta^{2} y_{1}\left(r_{1}^{i+1}, r_{2}^{i+2}\right),
\end{aligned}
$$


which implies

$$
y_{1}\left(r_{1}^{i}, r_{2}^{i+1}\right) \geq y_{1}\left(r_{1}^{i+1}, r_{2}^{i+2}\right) \Leftrightarrow y_{1}\left(r_{1}^{i}, r_{2}^{i+1}\right) \leq y_{1}^{1, \mathrm{I}} .
$$

The proof for $\omega \in \Omega, \omega \neq 1, \mathrm{I}$, is analogous.

The following lemma says that if the reference point pair is in $P_{\omega}$ now and at all future odd time points, then player 1 must propose $x^{\omega}$. A similar result holds for $Q_{\omega}$.

Lemma 2.A.4. If $\left(r_{1}^{k}, r_{2}^{k}\right) \in P_{\omega}$ for all $k \geq i$, then $x_{2}\left(r_{1}^{i}, r_{2}^{i}\right)=x_{2}^{\omega}$. Similarly, if $\left(r_{1}^{k}, r_{2}^{k+1}\right) \in Q_{\omega}$ for all $k \geq i$, then $y_{1}\left(r_{1}^{i}, r_{2}^{i+1}\right)=y_{1}^{\omega}$.

Proof. Observe that if $\left(r_{1}^{k}, r_{2}^{k}\right) \in P_{\omega}$ for all $k \geq i$, then by Lemma 2.A.2 it follows that $x_{2}\left(r_{1}^{i}, r_{2}^{i}\right)$ is either independent from the reference point pair $\left(r_{1}^{i}, r_{2}^{i}\right)$, or no offer is ever made that changes that reference point pair. Hence, $x_{2}\left(r_{1}^{i}, r_{2}^{i}\right)$ can be obtained as the sum of a geometric series. For instance, let $\left(r_{1}^{k}, r_{2}^{k}\right) \in P_{1, \mathrm{I}}$ for all $k \geq i$. Then by Lemma 2.A.2,

$$
\begin{aligned}
x_{2}\left(r_{1}^{i}, r_{2}^{i}\right) & =\delta-\delta^{2}+\delta^{2} x_{2}\left(r_{1}^{i+1}, r_{2}^{i+1}\right) \\
& =\delta(1-\delta)+\delta^{2}\left(\delta-\delta^{2}+\delta^{2} x_{2}\left(r_{1}^{i+2}, r_{2}^{i+2}\right)\right) \\
& \left.=\delta(1-\delta)\left(1+\delta^{2}\right)+\delta^{4} x_{2}\left(r_{1}^{i+2}, r_{2}^{i+2}\right)\right) \\
& =\delta(1-\delta)\left(1+\delta^{2}+\delta^{4}+\ldots\right) \\
& =\delta(1-\delta) \cdot \frac{1}{1-\delta^{2}} \\
& =x_{2}^{1, \mathrm{I}} .
\end{aligned}
$$

The proof for $P_{\omega}$ where $\omega \neq 1$,I and for $Q_{\omega}$ where $\omega \in \Omega$ is analogous to the proof for $P_{1, \mathrm{I}}$.

The following lemma establishes some restrictions on how reference points can move through the sets $P$ and $Q$.

Lemma 2.A.5. If $\left(r_{1}^{i}, r_{2}^{i}\right) \in P_{1, I I I}$, then $\left(r_{1}^{i}, r_{2}^{i+1}\right) \notin Q_{1, I}$.

If $\left(r_{1}^{i}, r_{2}^{i}\right) \in P_{2, I I I}$, then $\left(r_{1}^{i}, r_{2}^{i+1}\right) \notin Q_{2, I}$.

If $\left(r_{1}^{i}, r_{2}^{i+1}\right) \in Q_{3, I}$, then $\left(r_{1}^{i+1}, r_{2}^{i+1}\right) \notin P_{1, I}$.

If $\left(r_{1}^{i}, r_{2}^{i+1}\right) \in Q_{3, I I}$, then $\left(r_{1}^{i+1}, r_{2}^{i+1}\right) \notin P_{1, I I}$. 
Proof. Let $\left(r_{1}^{i}, r_{2}^{i}\right) \in P_{1, \mathrm{III}}$ and assume $\left(r_{1}^{i}, r_{2}^{i+1}\right) \in Q_{1, \mathrm{I}}$. Then from the definitions of $P_{1, \mathrm{III}}$ and $Q_{1, \mathrm{I}}$ we have

$$
\begin{aligned}
& y_{2}\left(r_{1}^{i}, r_{2}^{i+1}\right)>x_{2}\left(r_{1}^{i}, r_{2}^{i}\right) \geq r_{2}^{i}, \\
& r_{1}^{i}>x_{1}\left(r_{1}^{i+1}, r_{2}^{i+1}\right)>y_{1}\left(r_{1}^{i}, r_{2}^{i+1}\right), \text { and } \\
& r_{2}^{i+1}>y_{2}\left(r_{1}^{i+1}, r_{2}^{i+2}\right)>x_{2}\left(r_{1}^{i+1}, r_{2}^{i+1}\right) .
\end{aligned}
$$

Since $r_{1}^{i}>y_{1}\left(r_{1}^{i}, r_{2}^{i+1}\right)$ and $r_{2}^{i+1}>x_{2}\left(r_{1}^{i+1}, r_{2}^{i+1}\right)$ we have $r_{1}^{i+1}=r_{1}^{i}$ and $r_{2}^{i+2}=$ $r_{2}^{i+1}$ by (2.9). From (U1) it follows that

$$
y_{2}\left(r_{1}^{i}, r_{2}^{i+1}\right)=y_{2}\left(r_{1}^{i+1}, r_{2}^{i+2}\right) .
$$

Furthermore, since $x_{2}\left(r_{1}^{i}, r_{2}^{i}\right) \geq r_{2}^{i}$, (2.9) implies $r_{2}^{i+1}=x_{2}\left(r_{1}^{i}, r_{2}^{i}\right)$. Thus,

$$
r_{2}^{i+1}>y_{2}\left(r_{1}^{i+1}, r_{2}^{i+2}\right)=y_{2}\left(r_{1}^{i}, r_{2}^{i+1}\right)>x_{2}\left(r_{1}^{i}, r_{2}^{i}\right)=r_{2}^{i+1} \text {. }
$$

This is a contradiction.

The proofs of the other statements are analogous.

\section{The Proof of Theorem 2.4.1}

In this section we prove a series of lemmas which are used in the proof of Theorem 2.4.1.

Lemma 2.A.6. Let $\omega \in\{1, \mathrm{I}, 1, \mathrm{II}, 2, \mathrm{I}, 2, \mathrm{II}\}$. Then for all $\left(r_{1}^{i}, r_{2}^{i}\right) \in P_{\omega}$ we have $x\left(r_{1}^{i}, r_{2}^{i}\right)=x^{\omega}$. Similarly, for all $\left(r_{1}^{i}, r_{2}^{i+1}\right) \in Q_{\omega}$ we have $y\left(r_{1}^{i}, r_{2}^{i+1}\right)=y^{\omega}$.

Proof. Let $\left(r_{1}^{i}, r_{2}^{i}\right) \in P_{\omega}$ where $\omega \in\{1, \mathrm{I}, 1, \mathrm{II}, 2, \mathrm{I}, 2, \mathrm{II}\}$. Then $r_{1}^{i}>y_{1}\left(r_{1}^{i}, r_{2}^{i+1}\right)$ and $r_{2}^{i}>x_{2}\left(r_{1}^{i}, r_{2}^{i}\right)$. By (2.9) this implies $r_{1}^{i+1}=r_{1}^{i}$ and $r_{2}^{i+1}=r_{2}^{i}$. By (U1),

$$
x_{2}\left(r_{1}^{i}, r_{2}^{i}\right)=x_{2}\left(r_{1}^{i+1}, r_{2}^{i+1}\right) .
$$

By Lemma 2.A.3 $x_{2}\left(r_{1}^{i}, r_{2}^{i}\right)=x_{2}^{\omega}$. The case for $Q_{\omega}$ is similar.

Lemma 2.A.7. If $\left(r_{1}^{i}, r_{2}^{i+1}\right) \in Q_{3, I}$, then
i. $y_{1}\left(r_{1}^{i}, r_{2}^{i+1}\right) \geq y_{1}^{3, I}$.
ii. $y_{1}\left(r_{1}^{i}, r_{2}^{i+1}\right) \leq y_{1}^{3, I}$. 
Thus, $y_{1}\left(r_{1}^{i}, r_{2}^{i+1}\right)=y_{1}^{3, I}$.

Proof of Lemma 2.A.7, part $i$. Let $\left(r_{1}^{i}, r_{2}^{i+1}\right) \in Q_{3, \mathrm{I}}$. From the definition of $Q_{3, \text { I }}$ we have

$$
\begin{aligned}
& x_{1}\left(r_{1}^{i+1}, r_{2}^{i+1}\right)>y_{1}\left(r_{1}^{i}, r_{2}^{i+1}\right) \geq r_{1}^{i}, \text { and } \\
& r_{2}^{i+1}>y_{2}\left(r_{1}^{i+1}, r_{2}^{i+2}\right)>x_{2}\left(r_{1}^{i+1}, r_{2}^{i+1}\right) .
\end{aligned}
$$

Assume $y_{1}\left(r_{1}^{i}, r_{2}^{i+1}\right)<y_{1}^{3, \mathrm{I}}$. By Lemma 2.A.3, $y_{1}\left(r_{1}^{i}, r_{2}^{i+1}\right)>y_{1}\left(r_{1}^{i+1}, r_{2}^{i+2}\right)$. Since $y_{1}\left(r_{1}^{i}, r_{2}^{i+1}\right) \geq r_{1}^{i}$ we have from (2.9) that $r_{1}^{i+1}=y_{1}\left(r_{1}^{i}, r_{2}^{i+1}\right)$. Hence, $r_{1}^{i+1}>$ $y_{1}\left(r_{1}^{i+1}, r_{2}^{i+2}\right)$. We have two possibilities:

a. $r_{1}^{i+1}>x_{1}\left(r_{1}^{i+2}, r_{2}^{i+2}\right)>y_{1}\left(r_{1}^{i+1}, r_{2}^{i+2}\right)$, i.e. $\left(r_{1}^{i+1}, r_{2}^{i+1}\right) \in P_{1, \mathrm{I}}$.

b. $x_{1}\left(r_{1}^{i+2}, r_{2}^{i+2}\right) \geq r_{1}^{i+1}>y_{1}\left(r_{1}^{i+1}, r_{2}^{i+2}\right)$, i.e. $\left(r_{1}^{i+1}, r_{2}^{i+1}\right) \in P_{2, \mathrm{I}}$.

By Lemma 2.A.5, case a. can be ruled out. Hence, $\left(r_{1}^{i+1}, r_{2}^{i+1}\right) \in P_{2, \mathrm{I}}$. By Lemma 2.A.6, $x_{2}\left(r_{1}^{i+1}, r_{2}^{i+1}\right)=x_{2}^{2, \mathrm{I}}$. Since $\left(r_{1}^{i}, r_{2}^{i+1}\right) \in Q_{3, \mathrm{I}}$, Lemma 2.A.2 implies

$$
\begin{aligned}
y_{1}\left(r_{1}^{i}, r_{2}^{i+1}\right) & =\frac{\delta}{\mu_{1}}-\frac{\delta}{\mu_{1}} x_{2}\left(r_{1}^{i+1}, r_{2}^{i+1}\right) \\
& =\frac{\delta}{\mu_{1}}-\frac{\delta}{\mu_{1}} x_{2}^{2, \mathrm{I}} \\
& =\frac{\delta}{\mu_{1}} x_{1}^{2, \mathrm{I}} .
\end{aligned}
$$

By (2.9), we have $r_{1}^{i+1}=y_{1}\left(r_{1}^{i}, r_{2}^{i+1}\right)$. Note that $x_{1}^{2, \mathrm{I}}$ is a function of $r_{1}^{i+1}$, and therefore of $y_{1}\left(r_{1}^{i}, r_{2}^{i+1}\right)$. That is,

$$
\begin{aligned}
y_{1}\left(r_{1}^{i}, r_{2}^{i+1}\right) & =\frac{\delta}{\mu_{1}} \cdot \frac{\left(\mu_{1}-\delta\right)+\delta^{2} \lambda_{1} r_{1}^{i+1}}{\left(1+\lambda_{1}\right)-\delta^{2}} \\
& =\frac{\delta}{\mu_{1}} \cdot \frac{\left(\mu_{1}-\delta\right)+\delta^{2} \lambda_{1} y_{1}\left(r_{1}^{i}, r_{2}^{i+1}\right)}{\left(1+\lambda_{1}\right)-\delta^{2}}
\end{aligned}
$$


Solving for $y_{1}\left(r_{1}^{i}, r_{2}^{i+1}\right)$ yields

$$
\begin{aligned}
y_{1}\left(r_{1}^{i}, r_{2}^{i+1}\right) & =\frac{\delta}{\mu_{1}} \cdot \frac{\mu_{1}-\delta}{1+\lambda_{1}-\delta^{2}-\delta^{2} \lambda_{1} \frac{\delta}{\mu_{1}}} \\
& =\frac{\delta\left(\mu_{1}-\delta\right)}{\left(1+\lambda_{1}\right) \mu_{1}-\delta^{2} \mu_{1}-\delta^{3} \lambda_{1}} \\
& =\frac{\delta\left(\mu_{1}-\delta\right)}{\left(1+\lambda_{1}\right) \mu_{1}-\delta^{2}-\delta^{2} \lambda_{1}+\delta^{3} \lambda_{1}-\delta^{3} \lambda_{1}} \\
& =\frac{\delta\left(\mu_{1}-\delta\right)}{\left(1+\lambda_{1}\right)\left(\mu_{1}-\delta^{2}\right)} \\
& =\frac{\delta}{1+\lambda_{1}+\delta} \\
& =y_{1}^{3, \mathrm{I}},
\end{aligned}
$$

where we used the fact that

$$
\begin{aligned}
\left(1+\lambda_{1}\right)\left(\mu_{1}-\delta^{2}\right) & =\left(1+\lambda_{1}\right)\left(1+\lambda_{1}(1-\delta)-\delta^{2}\right) \\
& =\left(1+\lambda_{1}\right)\left((1+\delta)(1-\delta)+\lambda_{1}(1-\delta)\right) \\
& =\left(1+\lambda_{1}\right)(1-\delta)\left(1+\lambda_{1}+\delta\right) \\
& =\left(1-\delta+\lambda_{1}(1-\delta)\right)\left(1+\lambda_{1}+\delta\right) \\
& =\left(\mu_{1}-\delta\right)\left(1+\lambda_{1}+\delta\right) .
\end{aligned}
$$

Observe that $y_{1}\left(r_{1}^{i}, r_{2}^{i+1}\right)=y_{1}^{3, \mathrm{I}}$ is contradicting $y_{1}\left(r_{1}^{i}, r_{2}^{i+1}\right)<y_{1}^{3, \mathrm{I}}$, our initial assumption.

Before we can prove Part $i i$. we must make a similar argument for $Q_{3, \mathrm{II}}$.

Lemma 2.A.8. If $\left(r_{1}^{i}, r_{2}^{i+1}\right) \in Q_{3, I I}$, then

i. $y_{1}\left(r_{1}^{i}, r_{2}^{i+1}\right) \geq y_{1}^{3, I I}$.

ii. $y_{1}\left(r_{1}^{i}, r_{2}^{i+1}\right) \leq y_{1}^{3, I I}$.

Thus, $y_{1}\left(r_{1}^{i}, r_{2}^{i+1}\right)=y_{1}^{3, I I}$.

Proof of Lemma 2.A.8, Part $i$. Let $\left(r_{1}^{i}, r_{2}^{i+1}\right) \in Q_{3, \mathrm{II}}$. From the definition of $Q_{3, \mathrm{II}}$ it follows that

$$
\begin{aligned}
& x_{1}\left(r_{1}^{i+1}, r_{2}^{i+1}\right)>y_{1}\left(r_{1}^{i}, r_{2}^{i+1}\right) \geq r_{1}^{i}, \text { and } \\
& y_{2}\left(r_{1}^{i+1}, r_{2}^{i+1}\right) \geq r_{2}^{i+1}>x_{2}\left(r_{1}^{i+1}, r_{2}^{i+1}\right) .
\end{aligned}
$$


Assume $y_{1}\left(r_{1}^{i}, r_{2}^{i+1}\right)<y_{1}^{3, \text { II }}$. Thus, $y_{1}\left(r_{1}^{i}, r_{2}^{i+1}\right)>y_{1}\left(r_{1}^{i+1}, r_{2}^{i+2}\right)$ by Lemma 2.A.3. Since $y_{1}\left(r_{1}^{i}, r_{2}^{i+1}\right) \geq r_{1}^{i}$, we have $r_{1}^{i+1}=y_{1}\left(r_{1}^{i}, r_{2}^{i+1}\right)$ by (2.9). Hence, $r_{1}^{i+1}>$ $y_{1}\left(r_{1}^{i+1}, r_{2}^{i+2}\right)$, leaving two possibilities:

a. $r_{1}^{i+1} \geq x_{1}\left(r_{1}^{i+2}, r_{2}^{i+2}\right)>y_{1}\left(r_{1}^{i+1}, r_{2}^{i+2}\right)$, i.e. $\left(r_{1}^{i+1}, r_{2}^{i+1}\right) \in P_{1, \mathrm{II}}$.

b. $x_{1}\left(r_{1}^{i+2}, r_{2}^{i+2}\right)>r_{1}^{i+1}>y_{1}\left(r_{1}^{i+1}, r_{2}^{i+2}\right)$, i.e. $\left(r_{1}^{i+1}, r_{2}^{i+1}\right) \in P_{2, \mathrm{II}}$.

Case a. is ruled out by Lemma 2.A.5. Hence, $\left(r_{1}^{i+1}, r_{2}^{i+1}\right) \in P_{2, \mathrm{II}}$, and thus by Lemma 2.A.6, $x_{2}\left(r_{1}^{i+1}, r_{2}^{i+1}\right)=x_{2}^{2, \mathrm{II}}$. Since $\left(r_{1}^{i}, r_{2}^{i+1}\right) \in Q_{3, \mathrm{II}}$, we have from Lemma 2.A.2 that

$$
\begin{aligned}
y_{1}\left(r_{1}^{i}, r_{2}^{i+1}\right) & =\frac{\delta}{\mu_{1}}-\frac{\delta}{\mu_{1}} x_{2}\left(r_{1}^{i+1}, r_{2}^{i+1}\right) \\
& =\frac{\delta}{\mu_{1}}-\frac{\delta}{\mu_{1}} x_{2}^{2, \mathrm{II}} \\
& =\frac{\delta}{\mu_{1}} x_{1}^{2, \mathrm{II}} .
\end{aligned}
$$

Note that $y_{1}\left(r_{1}^{i}, r_{2}^{i+1}\right)=r_{1}^{i+1}$ and that $x_{1}^{2, \text { II }}$ is a function of $r_{1}^{i+1}$. That is,

$$
\begin{aligned}
y_{1}\left(r_{1}^{i}, r_{2}^{i+1}\right) & =\frac{\left(1+\lambda_{1}\right)\left(\left(\mu_{2}-\delta\right)+\delta \lambda_{2}\left(1-r_{2}^{i+1}\right)\right)+\delta^{2} \lambda_{1} r_{1}^{i+1}}{\left(1+\lambda_{1}\right)\left(1+\lambda_{2}\right)-\delta^{2}} \\
& =\frac{\left(1+\lambda_{1}\right)\left(\left(\mu_{2}-\delta\right)+\delta \lambda_{2}\left(1-r_{2}^{i+1}\right)\right)+\delta^{2} \lambda_{1} y_{1}\left(r_{1}^{i}, r_{2}^{i+1}\right)}{\left(1+\lambda_{1}\right)\left(1+\lambda_{2}\right)-\delta^{2}} .
\end{aligned}
$$

Solving for $y_{1}\left(r_{1}^{i}, r_{2}^{i+1}\right)$ yields

$$
\begin{aligned}
y_{1}\left(r_{1}^{i}, r_{2}^{i+1}\right) & =\frac{\delta}{\mu_{1}} \cdot \frac{\left(1+\lambda_{1}\right)\left(\mu_{2}-\delta+\delta \lambda_{2}\left(1-r_{2}^{i+1}\right)\right)}{\left(1+\lambda_{1}\right)\left(1+\lambda_{2}\right)-\delta^{2}-\delta^{2} \lambda_{1} \frac{\delta}{\mu_{1}}} \\
& =\frac{\delta\left(1+\lambda_{1}\right)\left(\mu_{2}-\delta+\delta \lambda_{2}\left(1-r_{2}^{i+1}\right)\right)}{\left(1+\lambda_{1}\right)\left(1+\lambda_{2}\right) \mu_{1}-\delta^{2} \mu_{1}-\delta^{3} \lambda_{1}} \\
& =\frac{\delta\left(1+\lambda_{1}\right)\left(\mu_{2}-\delta+\delta \lambda_{2}\left(1-r_{2}^{i+1}\right)\right)}{\left(1+\lambda_{1}\right)\left(1+\lambda_{2}\right) \mu_{1}-\delta^{2}-\delta^{2} \lambda_{1}+\delta^{3} \lambda_{1}-\delta^{3} \lambda_{1}} \\
& =\frac{\delta\left(1+\lambda_{1}\right)\left(\mu_{2}-\delta+\delta \lambda_{2}\left(1-r_{2}^{i+1}\right)\right)}{\left(1+\lambda_{1}\right)\left(\left(1+\lambda_{2}\right) \mu_{1}-\delta^{2}\right)} \\
& =\frac{\delta\left(\mu_{2}-\delta+\delta \lambda_{2}\left(1-r_{2}^{i+1}\right)\right)}{\left(1+\lambda_{2}\right) \mu_{1}-\delta^{2}} \\
& =y_{1}^{3, \mathrm{II}} .
\end{aligned}
$$


This contradicts $y_{1}\left(r_{1}^{i}, r_{2}^{i+1}\right)<y_{1}^{3, \text { II }}$.

This result allows us to prove the second part of Lemma 2.A.7.

Proof of Lemma 2.A.7, Part ii. Let $\left(r_{1}^{i}, r_{2}^{i+1}\right) \in Q_{3, \mathrm{I}}$. By the definition of $Q_{3, \text { I }}$ we have

$$
\begin{aligned}
& x_{1}\left(r_{1}^{i+1}, r_{2}^{i+1}\right)>y_{1}\left(r_{1}^{i}, r_{2}^{i+1}\right) \geq r_{1}^{i}, \text { and } \\
& r_{2}^{i+1}>y_{2}\left(r_{1}^{i+1}, r_{2}^{i+2}\right)>x_{2}\left(r_{1}^{i+1}, r_{2}^{i+1}\right) .
\end{aligned}
$$

Assume $y_{1}\left(r_{1}^{i}, r_{2}^{i+1}\right)>y_{1}^{3, \mathrm{I}}$. Then by Lemma 2 .A.3 we have $y_{1}\left(r_{1}^{i+1}, r_{2}^{i+2}\right)>$ $y_{1}\left(r_{1}^{i}, r_{2}^{i+1}\right)$. Observe that $y_{1}\left(r_{1}^{i}, r_{2}^{i+1}\right) \geq r_{1}^{i}$, and thus by (2.9) $y_{1}\left(r_{1}^{i}, r_{2}^{i+1}\right)=$ $r_{1}^{i+1}$. Hence, $y_{1}\left(r_{1}^{i+1}, r_{2}^{i+2}\right)>r_{1}^{i+1}$, implying $\left(r_{1}^{i+1}, r_{2}^{i+1}\right) \in P_{3, \mathrm{I}}$. Then from Lemma 2.A.2, $y_{1}\left(r_{1}^{i}, r_{2}^{i+1}\right)>y_{1}^{3, \mathrm{I}}$ and $y_{1}\left(r_{1}^{i+1}, r_{2}^{i+2}\right)>y_{1}\left(r_{1}^{i}, r_{2}^{i+1}\right)$, we have

$$
x_{2}\left(r_{1}^{i+1}, r_{2}^{i+1}\right)=\delta-\delta y_{1}\left(r_{1}^{i+1}, r_{2}^{i+2}\right)<\delta-\delta y_{1}\left(r_{1}^{i}, r_{2}^{i+1}\right)<\delta-\delta y_{1}^{3, \mathrm{I}}=x_{2}^{3, \mathrm{I}} .
$$

By Lemma 2.A.3 this implies $x_{2}\left(r_{1}^{i+1}, r_{2}^{i+1}\right)>x_{2}\left(r_{1}^{i+2}, r_{2}^{i+2}\right)$. Observe that, by (2.9), $r_{2}^{i+1}>x_{2}\left(r_{1}^{i+1}, r_{2}^{i+1}\right)$ implies $r_{2}^{i+2}=r_{2}^{i+1}$, and thus

$$
r_{2}^{i+2}=r_{2}^{i+1}>x_{2}\left(r_{1}^{i+1}, r_{2}^{i+1}\right)>x_{2}\left(r_{1}^{i+2}, r_{2}^{i+2}\right) .
$$

This leaves two possibilities.

a. $r_{2}^{i+2}>y_{2}\left(r_{1}^{i+2}, r_{2}^{i+3}\right)>x_{2}\left(r_{1}^{i+2}, r_{2}^{i+2}\right)$, i.e. $\left(r_{1}^{i+1}, r_{2}^{i+2}\right) \in Q_{3, \mathrm{I}}$.

b. $y_{2}\left(r_{1}^{i+2}, r_{2}^{i+3}\right) \geq r_{2}^{i+2}>x_{2}\left(r_{1}^{i+2}, r_{2}^{i+2}\right)$, i.e. $\left(r_{1}^{i+1}, r_{2}^{i+2}\right) \in Q_{3, \mathrm{II}}$.

Take case b., i.e. $\left(r_{1}^{i+1}, r_{2}^{i+2}\right) \in Q_{3, \mathrm{II}}$. Then

$$
y_{2}\left(r_{1}^{i+2}, r_{2}^{i+3}\right) \geq r_{2}^{i+2}=r_{2}^{i+1}>y_{2}\left(r_{1}^{i+1}, r_{2}^{i+2}\right),
$$

which implies $y_{1}\left(r_{1}^{i+1}, r_{2}^{i+2}\right)>y_{1}\left(r_{1}^{i+2}, r_{2}^{i+3}\right)$. By Lemma 2.A.3 we have $y_{1}\left(r_{1}^{i+1}\right.$, $\left.r_{2}^{i+2}\right)<y_{1}^{3, \text { II }}$. This contradicts part $i$. of Lemma 2.A.8.

Hence, case a. must hold, i.e. $\left(r_{1}^{i+1}, r_{2}^{i+2}\right) \in Q_{3, \mathrm{I}}$. Note that $x_{2}\left(r_{1}^{i+2}, r_{2}^{i+2}\right)<$ $x_{2}\left(r_{1}^{i+1}, r_{2}^{i+1}\right)<x_{2}^{3, \mathrm{I}}$. Then

$$
y_{1}\left(r_{1}^{i+1}, r_{2}^{i+2}\right)=\frac{\delta}{\mu_{1}}-\frac{\delta}{\mu_{1}} x_{2}\left(r_{1}^{i+2}, r_{2}^{i+2}\right)>\frac{\delta}{\mu_{1}}-\frac{\delta}{\mu_{1}} x_{2}^{3, \mathrm{I}}=y_{1}^{3, \mathrm{I}} .
$$

Thus, if $\left(r_{1}^{i}, r_{2}^{i+1}\right) \in Q_{3, \mathrm{I}}$ and $y_{1}\left(r_{1}^{i}, r_{2}^{i+1}\right)>y_{1}^{3, \mathrm{I}}$, then $\left(r_{1}^{i+1}, r_{2}^{i+2}\right) \in Q_{3, \mathrm{I}}$ and $y_{1}\left(r_{1}^{i+1}, r_{2}^{i+2}\right)>y_{1}^{3, \mathrm{I}}$. Thus, for all $k \geq i$ we have $\left(r_{1}^{k}, r_{2}^{k+1}\right) \in Q_{3, \mathrm{I}}$. Then by 
Lemma 2.A.4 we have that $y_{1}\left(r_{1}^{i}, r_{2}^{i+1}\right)=y_{1}^{3, \mathrm{I}}$. This contradicts $y_{1}\left(r_{1}^{i}, r_{2}^{i+1}\right)>$ $y_{1}^{3, \mathrm{I}}$.

We can now also complete the proof of Lemma 2.A.8.

Proof of Lemma 2.A.8, Part ii. Let $\left(r_{1}^{i}, r_{2}^{i+1}\right) \in Q_{3, \mathrm{II}}$. By definition of $Q_{3, \mathrm{II}}$ we have

$$
\begin{aligned}
& x_{1}\left(r_{1}^{i+1}, r_{2}^{i+1}\right)>y_{1}\left(r_{1}^{i}, r_{2}^{i+1}\right) \geq r_{1}^{i} \\
& y_{2}\left(r_{1}^{i+1}, r_{2}^{i+2}\right) \geq r_{2}^{i+1}>x_{2}\left(r_{1}^{i+1}, r_{2}^{i+1}\right) .
\end{aligned}
$$

Assume $y_{1}\left(r_{1}^{i}, r_{2}^{i+1}\right)>y_{1}^{3, \text { II }}$. Then by Lemma 2.A.3 we have $y_{1}\left(r_{1}^{i}, r_{2}^{i+1}\right)<$ $y_{1}\left(r_{1}^{i+1}, r_{2}^{i+2}\right)$. Since $y_{1}\left(r_{1}^{i}, r_{2}^{i+1}\right) \geq r_{1}^{i}$, (2.9) says that $r_{1}^{i+1}=y_{1}\left(r_{1}^{i}, r_{2}^{i+1}\right)$. Hence, $y_{1}\left(r_{1}^{i+1}, r_{2}^{i+2}\right)>r_{1}^{i+1}$, which implies $\left(r_{1}^{i+1}, r_{2}^{i+1}\right) \in P_{3, \mathrm{II}}$. From Lemma 2.A.2, $y_{1}\left(r_{1}^{i}, r_{2}^{i+1}\right)<y_{1}\left(r_{1}^{i+1}, r_{2}^{i+2}\right), y_{1}^{3, \mathrm{II}}<y_{1}\left(r_{1}^{i}, r_{2}^{i+1}\right)$, and the observation that $r_{2}^{i+2}=$ $\max \left\{r_{2}^{i+1}, x_{2}\left(r_{1}^{i+1}, r_{2}^{i+1}\right)\right\}=r_{2}^{i+1}$, it follows that

$$
\begin{aligned}
x_{2}\left(r_{1}^{i+1}, r_{2}^{i+1}\right)= & \frac{\delta\left(1+\lambda_{2} r_{2}^{i+2}\right)}{1+\lambda_{2}}-\frac{\delta}{1+\lambda_{2}} y_{1}\left(r_{1}^{i+1}, r_{2}^{i+2}\right) \\
& <\frac{\delta\left(1+\lambda_{2} r_{2}^{i+2}\right)}{1+\lambda_{2}}-\frac{\delta}{1+\lambda_{2}} y_{1}\left(r_{1}^{i}, r_{2}^{i+1}\right) \\
& <\frac{\delta\left(1+\lambda_{2} r_{2}^{i+2}\right)}{1+\lambda_{2}}-\frac{\delta}{1+\lambda_{2}} y_{1}^{3, \mathrm{II}} \\
& =\frac{\delta\left(1+\lambda_{2} r_{2}^{i+1}\right)}{1+\lambda_{2}}-\frac{\delta}{1+\lambda_{2}} y_{1}^{3, \mathrm{II}} \\
& =x_{2}^{3, \mathrm{II}} .
\end{aligned}
$$

By Lemma 2.A.3 this implies $x_{2}\left(r_{1}^{i+1}, r_{2}^{i+1}\right)>x_{2}\left(r_{1}^{i+2}, r_{2}^{i+2}\right)$. Since $r_{2}^{i+2}=r_{2}^{i+1}>$ $x_{2}\left(r_{1}^{i+1}, r_{2}^{i+1}\right)$, this implies $r_{2}^{i+2}>x_{2}\left(r_{1}^{i+2}, r_{2}^{i+2}\right)$. Then there are two possibilities:

a. $r_{2}^{i+2}>y_{2}\left(r_{1}^{i+2}, r_{2}^{i+3}\right)>x_{2}\left(r_{1}^{i+2}, r_{2}^{i+2}\right)$, i.e. $\left(r_{1}^{i+1}, r_{2}^{i+2}\right) \in Q_{3, \mathrm{I}}$.

b. $y_{2}\left(r_{1}^{i+2}, r_{2}^{i+3}\right) \geq r_{2}^{i+2}>x_{2}\left(r_{1}^{i+2}, r_{2}^{i+2}\right)$, i.e. $\left(r_{1}^{i+1}, r_{2}^{i+2}\right) \in Q_{3, \mathrm{II}}$.

Take case a., i.e. $\left(r_{1}^{i+1}, r_{2}^{i+2}\right) \in Q_{3, \mathrm{I}}$. Observe that

$$
y_{2}\left(r_{1}^{i+1}, r_{2}^{i+2}\right) \geq r_{2}^{i+1}=r_{2}^{i+2}>y_{2}\left(r_{1}^{i+2}, r_{2}^{i+3}\right)
$$


and thus $y_{1}\left(r_{1}^{i+1}, r_{2}^{i+2}\right)<y_{1}\left(r_{1}^{i+2}, r_{2}^{i+3}\right)$. By Lemma 2.A.3 we then obtain the inequality $y_{1}\left(r_{1}^{i+1}, r_{2}^{i+2}\right)>y_{1}^{3, \mathrm{I}}$. This contradicts part $i$. of Lemma 2.A.7.

Hence, case b. must hold, i.e. $\left(r_{1}^{i+1}, r_{2}^{i+2}\right) \in Q_{3, \text { II }}$. Since $x_{2}\left(r_{1}^{i+1}, r_{2}^{i+1}\right)>x_{2}^{3, I I}$ and $x_{2}\left(r_{1}^{i+2}, r_{2}^{i+2}\right)>x_{2}\left(r_{1}^{i+1}, r_{2}^{i+1}\right)$, we have from Lemma 2.A.2

$$
y_{1}\left(r_{1}^{i+1}, r_{2}^{i+2}\right)=\frac{\delta}{\mu_{1}}-\frac{\delta}{\mu_{1}} x_{2}\left(r_{1}^{i+2}, r_{2}^{i+2}\right)>\frac{\delta}{\mu_{1}}-\frac{\delta}{\mu_{1}} x_{2}^{3, \mathrm{II}}=y_{1}^{3, \mathrm{II}} .
$$

Thus, $\left(r_{1}^{i}, r_{2}^{i+1}\right) \in Q_{3, \mathrm{II}}$ and $y_{1}\left(r_{1}^{i}, r_{2}^{i+1}\right)>y_{1}^{3, \mathrm{II}}$ implies $\left(r_{1}^{i+1}, r_{2}^{i+2}\right) \in Q_{3, \mathrm{II}}$ and $y_{1}\left(r_{1}^{i+1}, r_{2}^{i+2}\right)>y_{1}^{3, \mathrm{II}}$. Then, for all $k \geq i$ we have $\left(r_{1}^{k}, r_{2}^{k+1}\right) \in Q_{3, \mathrm{II}}$, which by Lemma 2.A.4 implies $y_{1}\left(r_{1}^{i}, r_{2}^{i+1}\right)=y_{1}^{3, \text { II }}$. This contradicts $y_{1}\left(r_{1}^{i}, r_{2}^{i+1}\right)>$ $y_{1}^{3, \mathrm{II}}$.

Similar results hold in $P_{2, \mathrm{III}}$ and $P_{1, \mathrm{III}}$.

Lemma 2.A.9. If $\left(r_{1}^{i}, r_{2}^{i}\right) \in P_{1, I I I}$, then $x_{2}\left(r_{1}^{i}, r_{2}^{i}\right)=x_{2}^{1, I I I}$.

Lemma 2.A.10. If $\left(r_{1}^{i}, r_{2}^{i}\right) \in P_{2, I I I}$, then $x_{2}\left(r_{1}^{i}, r_{2}^{i}\right)=x_{2}^{2, I I I}$.

The proofs of these lemmas are analogous to the proofs of Lemmas 2.A.7 and 2.A.8 respectively.

Lemma 2.A.11. If $\left(r_{1}^{i}, r_{2}^{i}\right) \in P_{3, I I I}$, then

$$
\begin{aligned}
& \text { i. } x_{2}\left(r_{1}^{i}, r_{2}^{i}\right) \geq x_{2}^{3, I I I} . \\
& \text { ii. } x_{2}\left(r_{1}^{i}, r_{2}^{i}\right) \leq x_{2}^{3, I I I} .
\end{aligned}
$$

Thus, $x_{2}\left(r_{1}^{i}, r_{2}^{i}\right)=x_{2}^{3, I I I}$.

Proof of Lemma 2.A.11, Part $i$. Let $\left(r_{1}^{i}, r_{2}^{i}\right) \in P_{3, \mathrm{III}}$. By the definition of $P_{3, \text { III }}$ we have

$$
\begin{aligned}
& y_{2}\left(r_{1}^{i}, r_{2}^{i+1}\right)>x_{2}\left(r_{1}^{i}, r_{2}^{i}\right) \geq r_{2}^{i}, \\
& x_{1}\left(r_{1}^{i+1}, r_{2}^{i+1}\right)>y_{1}\left(r_{1}^{i}, r_{2}^{i+1}\right) \geq r_{1}^{i} .
\end{aligned}
$$

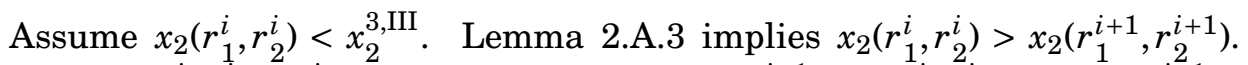
Since $x_{2}\left(r_{1}^{i}, r_{2}^{i}\right) \geq r_{2}^{i}$, we have from (2.9) that $r_{2}^{i+1}=x_{2}\left(r_{1}^{i}, r_{2}^{i}\right)$. Hence, $r_{2}^{i+1}>$ $x_{2}\left(r_{1}^{i+1}, r_{2}^{i+1}\right)$. Then there are two possibilities:

a. $r_{2}^{i+1}>y_{2}\left(r_{1}^{i+1}, r_{2}^{i+2}\right)>x_{2}\left(r_{1}^{i+1}, r_{2}^{i+1}\right)$, i.e. $\left(r_{1}^{i}, r_{2}^{i+1}\right) \in Q_{3, \mathrm{I}}$. 
b. $y_{2}\left(r_{1}^{i+1}, r_{2}^{i+2}\right) \geq r_{2}^{i+1}>x_{2}\left(r_{1}^{i+1}, r_{2}^{i+1}\right)$, i.e. $\left(r_{1}^{i}, r_{2}^{i+1}\right) \in Q_{3, \mathrm{II}}$.

Take case a., i.e. $\left(r_{1}^{i}, r_{2}^{i+1}\right) \in Q_{3, \mathrm{I}}$. From Lemma 2.A.7 we have $y_{1}\left(r_{1}^{i}, r_{2}^{i+1}\right)=$ $y_{1}^{3, \mathrm{I}}$. From Lemma 2.A.2 it follows that $x_{2}\left(r_{1}^{i}, r_{2}^{i}\right)=\frac{\delta}{\mu_{2}}-\frac{\delta}{\mu_{2}} y_{1}^{3, \mathrm{I}}=\frac{\delta}{\mu_{2}} y_{2}^{3, \mathrm{I}}$. Observe that $\mu_{2}\left(\mu_{1}-\delta^{2}\right)<\mu_{1} \mu_{2}-\delta^{2}$. Thus,

$$
\begin{aligned}
& y_{2}^{3, \mathrm{I}}=\frac{1+\lambda_{1}}{1+\lambda_{1}+\delta}=\frac{\left(1+\lambda_{1}\right)(1-\delta)}{\left(1+\lambda_{1}+\delta\right)(1-\delta)}=\frac{\left(1-\delta+\lambda_{1}(1-\delta)\right)}{1-\delta+\lambda_{1}(1-\delta)+\delta-\delta^{2}} \\
& =\frac{\mu_{2}}{\mu_{2}} \cdot \frac{\left(\mu_{1}-\delta\right)}{\mu_{1}-\delta^{2}}>\frac{\mu_{2}\left(\mu_{1}-\delta\right)}{\mu_{1} \mu_{2}-\delta^{2}}=y_{2}^{3, \mathrm{III}} .
\end{aligned}
$$

Then $x_{2}\left(r_{1}^{i}, r_{2}^{i}\right)>\frac{\delta}{\mu_{2}} y_{2}^{3, \text { III }}=x_{2}^{3, \text { III }}$, contradicting the initial assumption $x_{2}\left(r_{1}^{i}\right.$, $\left.r_{2}^{i}\right)<x_{2}^{3, \text { III }}$.

Take case b., i.e., $\left(r_{1}^{i}, r_{2}^{i+1}\right) \in Q_{3, \mathrm{II}}$. By Lemma 2.A.2, $x_{2}\left(r_{1}^{i}, r_{2}^{i}\right)=\frac{\delta}{\mu_{2}} y_{2}^{3, \mathrm{II}}$. Note that $r_{2}^{i+1}=x_{2}\left(r_{1}^{i}, r_{2}^{i}\right)$ by (2.9), and that $y_{2}^{3, \text { II }}$ is a function of $r_{2}^{i+1}$. That is,

$$
\begin{aligned}
x_{2}\left(r_{1}^{i}, r_{2}^{i}\right) & =\frac{\delta}{\mu_{2}} \cdot \frac{\left(\mu_{1}-\delta\right)\left(1+\lambda_{2}\right)+\delta^{2} \lambda_{2} r_{2}^{i+1}}{\mu_{1}\left(1+\lambda_{2}\right)-\delta^{2}} \\
& =\frac{\delta}{\mu_{2}} \cdot \frac{\left(\mu_{1}-\delta\right)\left(1+\lambda_{2}\right)+\delta^{2} \lambda_{2} x_{2}\left(r_{1}^{i}, r_{2}^{i}\right)}{\mu_{1}\left(1+\lambda_{2}\right)-\delta^{2}} .
\end{aligned}
$$

Solving for $x_{2}\left(r_{1}^{i}, r_{2}^{i}\right)$ yields

$$
\begin{aligned}
& x_{2}\left(r_{1}^{i}, r_{2}^{i}\right)=\frac{\delta}{\mu_{2}} \cdot \frac{\left(\mu_{1}-\delta\right)\left(1+\lambda_{2}\right)}{\mu_{1}\left(1+\lambda_{2}\right)-\delta^{2}-\delta^{2} \lambda_{2} \frac{\delta}{\mu_{2}}}=\frac{\delta\left(\mu_{1}-\delta\right)\left(1+\lambda_{2}\right)}{\mu_{1} \mu_{2}\left(1+\lambda_{2}\right)-\delta^{2} \mu_{2}-\delta^{3} \lambda_{2}} \\
& =\frac{\delta\left(\mu_{1}-\delta\right)\left(1+\lambda_{2}\right)}{\mu_{1} \mu_{2}\left(1+\lambda_{2}\right)-\delta^{2}-\delta^{2} \lambda_{2}+\delta^{3} \lambda_{2}-\delta^{3} \lambda_{2}}=\frac{\delta\left(\mu_{1}-\delta\right)}{\mu_{1} \mu_{2}-\delta^{2}}=x_{2}^{3, \mathrm{III}} .
\end{aligned}
$$

This contradicts $x_{2}\left(r_{1}^{i}, r_{2}^{i}\right)<x_{2}^{3, \text { III }}$.

Before we can complete the proof of Lemma 2.A.11, we need a similar result for $Q_{3, \mathrm{III}}$.

Lemma 2.A.12. If $\left(r_{1}^{i}, r_{2}^{i+1}\right) \in Q_{3, I I I}$, then
i. $y_{1}\left(r_{1}^{i}, r_{2}^{i+1}\right) \geq y_{1}^{3, I I I}$,
ii. $y_{1}\left(r_{1}^{i}, r_{2}^{i+1}\right) \leq y_{1}^{3, I I I}$. 
Thus, $y_{1}\left(r_{1}^{i}, r_{2}^{i+1}\right)=y_{1}^{3, I I I}$.

Proof of Lemma 2.A.12, Part $i$. This is analogous to the proof of Lemma 2.A.11 Part $i$.

We continue with the second part of Lemma 2.A.11.

Proof of Lemma 2.A.11, Part ii. Let $\left(r_{1}^{i}, r_{2}^{i}\right) \in P_{3, \mathrm{III}}$. By definition of $P_{3, \mathrm{III}}$ we have

$$
\begin{aligned}
& y_{2}\left(r_{1}^{i}, r_{2}^{i+1}\right)>x_{2}\left(r_{1}^{i}, r_{2}^{i}\right) \geq r_{2}^{i}, \text { and } \\
& x_{1}\left(r_{1}^{i+1}, r_{2}^{i+1}\right)>y_{1}\left(r_{1}^{i}, r_{2}^{i+1}\right) \geq r_{1}^{i} .
\end{aligned}
$$

Assume $x_{2}\left(r_{1}^{i}, r_{2}^{i}\right)>x_{2}^{3, \text { III }}$. Then by Lemma 2.A.3 $x_{2}\left(r_{1}^{i}, r_{2}^{i}\right)<x_{2}\left(r_{1}^{i+1}, r_{2}^{i+1}\right)$. Since $x_{2}\left(r_{1}^{i}, r_{2}^{i}\right) \geq r_{2}^{i}$, we have $r_{2}^{i+1}=x_{2}\left(r_{1}^{i}, r_{2}^{i}\right)$ by (2.9). Thus, $r_{2}^{i+1}<x_{2}\left(r_{1}^{i+1}\right.$, $r_{2}^{i+1}$ ), implying $\left(r_{1}^{i}, r_{2}^{i+1}\right) \in Q_{3, \mathrm{III}}$. By the first part of Lemma 2.A.12 this implies that $y_{1}\left(r_{1}^{i}, r_{2}^{i+1}\right) \geq y_{1}^{3, \mathrm{III}}$. Then from Lemma 2.A.2 and the construction of $x^{3, \mathrm{III}}$ we obtain

$$
x_{2}\left(r_{1}^{i}, r_{2}^{i}\right)=\frac{\delta}{\mu_{2}}-\frac{\delta}{\mu_{2}} y_{1}\left(r_{1}^{i}, r_{2}^{i+1}\right) \leq \frac{\delta}{\mu_{2}}-\frac{\delta}{\mu_{2}} y_{1}^{3, \text { III }}=x_{2}^{3, \text { III }} .
$$

This contradicts $x_{2}\left(r_{1}^{i}, r_{2}^{i}\right)>x_{2}^{3, \text { III }}$.

In a similar way we can complete the proof of Lemma 2.A.12.

Proof of Lemma 2.A.12, Part ii. Analogous to the proof of Lemma 2.A.11, Part ii.

We can now obtain the offers made in the sets $P_{3, \mathrm{I}}$ and $P_{3, \mathrm{II}}$.

Lemma 2.A.13. If $\left(r_{1}^{i}, r_{2}^{i}\right) \in P_{3, I}$ then $x_{2}\left(r_{1}^{i}, r_{2}^{i}\right)=x_{2}^{3, I}$.

Proof. Let $\left(r_{1}^{i}, r_{2}^{i}\right) \in P_{3, \mathrm{I}}$. By definition of $P_{3, \mathrm{I}}$ we have

$$
\begin{aligned}
& r_{2}^{i}>y_{2}\left(r_{1}^{i}, r_{2}^{i+1}\right)>x_{2}\left(r_{1}^{i}, r_{2}^{i}\right), \text { and } \\
& x_{1}\left(r_{1}^{i+1}, r_{2}^{i+1}\right)>y_{1}\left(r_{1}^{i}, r_{2}^{i+1}\right) \geq r_{1}^{i} .
\end{aligned}
$$

Assume $x_{2}\left(r_{1}^{i}, r_{2}^{i}\right) \neq x_{2}^{3, \mathrm{I}}$. We have three mutually exclusive and exhaustive cases. 
- $\left(r_{1}^{i}, r_{2}^{i+1}\right) \in Q_{3, \mathrm{I}}$ : Observe that by Lemma 2.A.2, $\left(r_{1}^{i}, r_{2}^{i}\right) \in P_{3, \mathrm{I}}$ implies $x_{2}\left(r_{1}^{i}, r_{2}^{i}\right)=\delta-\delta y_{1}\left(r_{1}^{i}, r_{2}^{i+1}\right)=\delta y_{2}\left(r_{1}^{i}, r_{2}^{i+1}\right)$. Then by Lemma 2.A.7 and the construction of $x^{3, \mathrm{I}}$ this implies $x_{2}\left(r_{1}^{i}, r_{2}^{i}\right)=\delta y_{2}^{3, \mathrm{I}}=x_{2}^{3, \mathrm{I}}$, contradicting the assumption that $x_{2}\left(r_{1}^{i}, r_{2}^{i}\right) \neq x_{2}^{3, \mathrm{I}}$.

- $\left(r_{1}^{i}, r_{2}^{i+1}\right) \in Q_{3, \mathrm{II}}$ : Since $\left(r_{1}^{i}, r_{2}^{i}\right) \in P_{3, \mathrm{I}}$ and $\left(r_{1}^{i}, r_{2}^{i+1}\right) \in Q_{3, \mathrm{II}}$ we have

$$
y_{2}\left(r_{1}^{i+1}, r_{2}^{i+2}\right) \geq r_{2}^{i+1}=\max \left\{r_{2}^{i}, x_{2}\left(r_{1}^{i}, r_{2}^{i}\right)\right\}=r_{2}^{i}>y_{2}\left(r_{1}^{i}, r_{2}^{i+1}\right) .
$$

However, by Lemma 2.A.8 we have $y_{2}\left(r_{1}^{i}, r_{2}^{i+1}\right)=y_{2}^{3, \text { II }}$, which implies $y_{2}\left(r_{1}^{i}, r_{2}^{i+1}\right)=y_{2}\left(r_{1}^{i+1}, r_{2}^{i+2}\right)$ by Lemma 2.A.3. This contradicts the above.

- $\left(r_{1}^{i}, r_{2}^{i+1}\right) \in Q_{3, \mathrm{III}}:$ By Lemma 2.A.2, Lemma 2.A.12, inequality (2.11), and the construction of $x^{3, \mathrm{I}}$, we have

$$
x_{2}\left(r_{1}^{i}, r_{2}^{i}\right)=\delta-\delta y_{1}\left(r_{1}^{i}, r_{2}^{i+1}\right)=\delta y_{2}^{3, \text { III }}<\delta y_{2}^{3, \mathrm{I}}=x_{2}^{3, \mathrm{I}} .
$$

By Lemma 2.A.3 this implies $x_{2}\left(r_{1}^{i}, r_{2}^{i}\right)>x_{2}\left(r_{1}^{i+1}, r_{2}^{i+1}\right)$. Since $r_{2}^{i}>$ $x_{2}\left(r_{1}^{i}, r_{2}^{i}\right)$ we have by (2.9) that $r_{2}^{i+1}=r_{2}^{i}$, implying $r_{2}^{i+1}>x_{2}\left(r_{1}^{i+1}, r_{2}^{i+1}\right)$. This implies $\left(r_{1}^{i}, r_{2}^{i+1}\right) \notin Q_{3, \mathrm{III}}$, a contradiction.

It follows that $x_{2}\left(r_{1}^{i}, r_{2}^{i}\right)=x_{2}^{3, \mathrm{I}}$.

Lemma 2.A.14. If $\left(r_{1}^{i}, r_{2}^{i}\right) \in P_{3, I I}$ then $x_{2}\left(r_{1}^{i}, r_{2}^{i}\right)=x_{2}^{3, I I}$.

Proof. Let $\left(r_{1}^{i}, r_{2}^{i}\right) \in P_{3, \mathrm{II}}$. By definition of $P_{3, \mathrm{II}}$ we have

$$
\begin{aligned}
& y_{2}\left(r_{1}^{i}, r_{2}^{i+1}\right) \geq r_{2}^{i}>x_{2}\left(r_{1}^{i}, r_{2}^{i}\right) \\
& x_{1}\left(r_{1}^{i+1}, r_{2}^{i+1}\right)>y_{1}\left(r_{1}^{i}, r_{2}^{i+1}\right) \geq r_{1}^{i} .
\end{aligned}
$$

Assume $x_{2}\left(r_{1}^{i}, r_{2}^{i}\right) \neq x_{2}^{3, \text { II }}$. Then we distinguish three mutually exclusive and exhaustive cases.

- $\left(r_{1}^{i}, r_{2}^{i+1}\right) \in Q_{3, \mathrm{I}}$ : Since $r_{2}^{i}>x_{2}\left(r_{1}^{i}, r_{2}^{i}\right)$ we have from (2.9) that $r_{2}^{i}=r_{2}^{i+1}$. Hence,

$$
y_{2}\left(r_{1}^{i}, r_{2}^{i+1}\right) \geq r_{2}^{i}=r_{2}^{i+1}>y_{2}\left(r_{1}^{i+1}, r_{2}^{i+2}\right) .
$$

However, by Lemma 2.A.7 we have $y_{2}\left(r_{1}^{i}, r_{2}^{i+1}\right)=y_{2}^{3, \mathrm{I}}$, which by Lemma 2.A.3 implies $y_{2}\left(r_{1}^{i}, r_{2}^{i+1}\right)=y_{2}\left(r_{1}^{i+1}, r_{2}^{i+2}\right)$. This contradicts the above. 
- $\left(r_{1}^{i}, r_{2}^{i+1}\right) \in Q_{3, \text { II }}$ : From Lemma 2.A.8 we obtain $y_{1}\left(r_{1}^{i}, r_{2}^{i+1}\right)=y_{1}^{3, \mathrm{II}}$. By Lemma 2.A.2 and the construction of $x^{3, \mathrm{II}}$, we then have

$$
\begin{aligned}
x_{2}\left(r_{1}^{i}, r_{2}^{i}\right) & =\frac{\delta\left(1+\lambda_{2} r_{2}^{i+1}\right)}{1+\lambda_{2}}-\frac{\delta y_{1}\left(r_{1}^{i}, r_{2}^{i+1}\right)}{1+\lambda_{2}} \\
& =\frac{\delta\left(1+\lambda_{2} r_{2}^{i+1}\right)}{1+\lambda_{2}}-\frac{\delta y_{1}^{3, \mathrm{II}}}{1+\lambda_{2}} \\
& =\frac{\delta\left(y_{2}^{3, \mathrm{II}}+\lambda_{2} r_{2}^{i+1}\right)}{1+\lambda_{2}} \\
& =x_{2}^{3, \mathrm{II}},
\end{aligned}
$$

contradicting $x_{2}\left(r_{1}^{i}, r_{2}^{i}\right) \neq x_{2}^{3, \text { II }}$.

- $\left(r_{1}^{i}, r_{2}^{i+1}\right) \in Q_{3, \mathrm{III}}$ : By Lemma 2.A.12 and the definition of $y_{1}^{3, \mathrm{III}}$ this implies

$$
y_{1}\left(r_{1}^{i}, r_{2}^{i+1}\right)=y_{1}^{3, \mathrm{III}}=\frac{\delta}{\mu_{1}}-\frac{\delta}{\mu_{1}} x_{2}^{3, \mathrm{III}} .
$$

By Lemma 2.A.2 we have

$$
y_{1}\left(r_{1}^{i}, r_{2}^{i+1}\right)=\frac{\delta}{\mu_{1}}-\frac{\delta}{\mu_{1}} x_{2}\left(r_{1}^{i+1}, r_{2}^{i+1}\right) .
$$

Hence, $x_{2}\left(r_{1}^{i+1}, r_{2}^{i+1}\right)=x_{2}^{3, \text { III }}$.

Since $r_{2}^{i}>x_{2}\left(r_{1}^{i}, r_{2}^{i}\right)$, we have from (2.9) that $r_{2}^{i+1}=r_{2}^{i}$. Then

$$
x_{2}\left(r_{1}^{i+1}, r_{2}^{i+1}\right) \geq r_{2}^{i+1}=r_{2}^{i}>x_{2}\left(r_{1}^{i}, r_{2}^{i}\right) .
$$

By Lemma 2.A.3 this implies $x_{2}\left(r_{1}^{i}, r_{2}^{i}\right)>x_{2}^{3, \text { II }}$. Since $r_{2}^{i}>x_{2}\left(r_{1}^{i}, r_{2}^{i}\right)$ and $r_{2}^{i}=r_{2}^{i+1}$ this implies $r_{2}^{i+1}>x_{2}^{3, \text { II }}$. That is,

$$
r_{2}^{i+1}>\frac{\delta\left(\mu_{1}-\delta+\lambda_{2} r_{2}^{i+1} \mu_{1}\right)}{\mu_{1}\left(1+\lambda_{2}\right)-\delta^{2}} .
$$

This is equivalent to $r_{2}^{i+1}>\frac{\delta\left(\mu_{1}-\delta\right)}{\mu_{1} \mu_{2}-\delta^{2}}=x_{2}^{3, \text { III }}$. Hence, $r_{2}^{i+1}>x_{2}\left(r_{1}^{i+1}, r_{2}^{i+1}\right)$, which implies $\left(r_{1}^{i}, r_{2}^{i+1}\right) \notin Q_{3, \mathrm{III}}$. This is a contradiction.

It follows that $x_{2}\left(r_{1}^{i}, r_{2}^{i}\right)=x_{2}^{3, \text { II }}$.

Similar results hold for $Q_{2, \mathrm{III}}$ and $Q_{1, \mathrm{III}}$. 
Lemma 2.A.15. If $\left(r_{1}^{i}, r_{2}^{i+1}\right) \in Q_{1, I I I}$ then $y_{1}\left(r_{1}^{i}, r_{2}^{i+1}\right)=y_{1}^{1, I I I}$.

Lemma 2.A.16. If $\left(r_{1}^{i}, r_{2}^{i+1}\right) \in Q_{2, I I I}$ then $y_{1}\left(r_{1}^{i}, r_{2}^{i+1}\right)=y_{1}^{2, I I I}$.

Proofs are analogous to Lemma 2.A.13 resp. 2.A.14. We now prove the main result of this section.

Proof of Theorem 2.4.1. Let $\left(r_{1}^{i}, r_{2}^{i}\right)=P_{1, \mathrm{I}}$. By definition we have $r_{2}^{i}>$ $y_{2}\left(r_{1}^{i}, r_{2}^{i+1}\right)$ and $r_{1}^{i}>x_{1}\left(r_{1}^{i}, r_{2}^{i}\right)$. By Lemma 2.A.6 this implies $r_{2}^{i}>y_{2}^{1, \mathrm{I}}$ and $r_{1}^{i}>x_{1}^{1, \mathrm{I}}$. Hence, $\left(r_{1}^{i}, r_{2}^{i}\right) \in X_{1, \mathrm{I}}$, from which it follows that $P_{1, \mathrm{I}} \subseteq X_{1, \mathrm{I}}$.

Similarly, by Lemmas 2.A.6, 2.A.9, 2.A.10, 2.A.11, 2.A.13, and 2.A.14, $\left(r_{1}^{i}, r_{2}^{i}\right) \in P_{\omega}$ implies $\left(r_{1}^{i}, r_{2}^{i}\right) \in X_{\omega}$ for each $\omega \in \Omega$. Hence, $P_{\omega} \subseteq X_{\omega}$ for each $\omega \in \Omega$. Since the sets $P_{\omega}$ are mutually exclusive and exhaustive, this implies

$$
P_{\omega}=X_{\omega} \text { for each } \omega \in \Omega \text {. }
$$

Hence, at times $t \in T_{\text {odd }}$ player 1's unique SPE strategy is to propose $x^{\omega}$ if $\left(r_{1}, r_{2}\right) \in X_{\omega}$. That is, to play strategy $\hat{f}$. Analogously, we have

$$
Q_{\omega}=X_{\omega} \text { for each } \omega \in \Omega,
$$

such that player 2's unique SPE strategy at times $t \in T_{\text {even }}$ is to propose $y^{\omega}$ if $\left(r_{1}, r_{2}\right) \in X_{\omega}$, i.e. to follow strategy $\hat{g}$.

It follows from part B. of the proof of Theorem 2.3.1 that player 1's unique optimal strategy at times $t \in T_{\text {even }}$ is to accept proposals which are at or above 2's SPE proposal, and to reject those which are not. (Rejecting player 2's proposal violates immediate acceptance.) That is, player 1's unique SPE strategy at times $t \in T_{\text {even }}$ is $\hat{f}$. Similarly, player 2's unique optimal strategy at times $t \in T_{o d d}$ is to accept and reject proposals according to the strategy $\hat{g}$.

Thus, the strategy profile $(\hat{f}, \hat{g})$ is the unique SPE satisfying (U1)-(U3).

\section{A.3 Concave Utility Functions}

Let player $i$ 's preference for pie, $i=1,2$, be represented by a strictly increasing, concave von Neumann-Morgenstern utility function $v_{i}:[0,1] \rightarrow \mathbb{R}$. Since such a utility function is unique up to an affine transformation, we may assume that $v_{i}(0)=0$ and $v_{i}(1)=1$. Furthermore, define

$$
\tilde{Z}:=\left\{\left(v_{1}\left(z_{1}\right), v_{2}\left(z_{2}\right)\right) \mid z \in Z\right\} .
$$


In this more general setting, each finite path in $\bar{H}$ is associated with a utility outcome. That is, for all $h \in \bar{H} \backslash H^{\infty}$, we say that player $i, i=1,2$, obtains $v_{i}\left(\xi_{i}(h)\right)$, rather than $\xi_{i}(h)$. Note that due to rescaling, both players still attach a zero utility payoff to a disagreement path, and a utility payoff of one to agreement on a partition that gives them the whole pie. The loss aversion transformation $w$ is then applied to the utility outcome $v_{i}\left(\xi_{i}(h)\right)$. The reference point $r_{i}$ used in this transformation, is defined as the utility associated with the highest share player $i$ has rejected in the past. Like before, we have

a. $r_{1}>x_{1}>y_{1}: \delta_{1} x_{1}=y_{1}$.

b. $x_{1} \geq r_{1}>y_{1}: \delta_{1} x_{1}=\left(1+\lambda_{1}\right) y_{1}-\delta_{1} \lambda_{1} r_{1}$.

c. $x_{1}>y_{1} \geq r_{1}: \delta_{1} x_{1}=\mu_{1} y_{1}$.

and

i. $r_{2}>y_{2}>x_{2}: \delta_{2} y_{2}=x_{2}$.

ii. $y_{2} \geq r_{2}>x_{2}: \delta_{2} y_{2}=\left(1+\lambda_{2}\right) x_{2}-\delta_{2} \lambda_{2} r_{2}$.

iii. $y_{2}>x_{2} \geq r_{2}: \delta_{2} y_{2}=\mu_{2} x_{2}$.

where $x, y \in \tilde{Z}$. These equations again define a ninefold partition of the set of all possible reference point pairs, similar to the one depicted in Figure 2.1. Define $\tilde{\Omega}:=$ a.i.,.,., c.iii. $\}$, and denote the associated sets again by $X_{\omega}$, $\omega \in \tilde{\Omega}$. It can be shown that the system of equations yields a unique solution $\left(x^{\omega}, y^{\omega}\right)$ in $\tilde{Z} \times \tilde{Z}$ for each $\omega \in \tilde{\Omega}$. Let $h^{t}=\left(h^{t-1}, z\right)$ with $z \in \tilde{Z}$. Then for all $\omega \in \tilde{\Omega}$, define the strategy pair $(\breve{f}, \breve{g})$ for $t \in T_{\text {odd }}$ by

$$
\breve{f}^{t}\left(h^{t-1}\right)=x^{\omega}, \quad \breve{g}^{t}\left(h^{t}\right)=\left\{\begin{array}{ll}
\mathrm{Y} & \text { if } z_{2} \geq x_{2}^{\omega} \\
\mathrm{N} & \text { otherwise. }
\end{array}, \quad \text { if }\left(r_{1}\left(h^{t-1}\right), r_{2}\left(h^{t-1}\right)\right) \in X_{\omega},\right.
$$

and for $t \in T_{\text {even }}$ by

$$
\breve{f}^{t}\left(h^{t}\right)=\left\{\begin{array}{ll}
\mathrm{Y} & \text { if } z_{1} \geq y_{1}^{\omega} \\
\mathrm{N} & \text { otherwise }
\end{array}, \quad \breve{g}^{t}\left(h^{t-1}\right)=y^{\omega}, \quad \text { if }\left(r_{1}\left(h^{t-1}\right), r_{2}\left(h^{t-1}\right)\right) \in X_{\omega} .\right.
$$

We obtain the following result. 
Theorem 2.A.17. The strategy profile $(\breve{f}, \breve{g})$ is an SPE.

Proof. Observe that Lemma 2.A.1 continues to hold if players' utility functions are concave. Hence, the game is still continuous at infinity. The proof of Theorem 2.A.17 is then analogous to that of Theorem 2.3.1.

It follows that the partition obtained at the beginning of the game, where reference points are zero, is given by $x^{\text {c.iii. }}$. Henceforth, we drop the superscript c.iii., and write the solution as $x$. Note that we may again generalize the above results to the case where the time lapse between proposals is $\Delta$. Thus, we have

$$
\psi\left(x_{1}\right)=x_{2}=\frac{\delta_{2}^{\Delta}}{\mu_{2}} y_{2}^{\text {c.iii. }}=\frac{\delta_{2}^{\Delta}}{\mu_{2}} \psi\left(y_{1}^{\text {c.iii. }}\right)=\frac{\delta_{2}^{\Delta}}{\mu_{2}} \psi\left(\frac{\delta_{1}^{\Delta}}{\mu_{1}} x_{1}\right)
$$

where $\mu_{i}=1+\lambda_{i}\left(1-\delta_{i}^{\Delta}\right)$. Then by extending Proposition 4 from Binmore et al. (1986), we generalize the result that the equilibrium partition converges to an asymmetric Nash bargaining solution partition as $\Delta$ goes to zero, to the case where players have concave utility functions.

Let $z^{N}$ be the asymmetric Nash solution

$$
z^{N}=\underset{z \in \tilde{Z}}{\arg \max } z_{1}^{\alpha} z_{2}^{1-\alpha}
$$

where $\alpha=\frac{\left(1+\lambda_{2}\right) \log \delta_{2}}{\left(1+\lambda_{1}\right) \log \delta_{1}+\left(1+\lambda_{2}\right) \log \delta_{2}}$. Observe that $z^{N}$ is uniquely defined by

- $z_{2}^{N}=\psi\left(z_{1}^{N}\right)$, and

- $\left|\psi_{-}^{\prime}\left(z_{1}^{N}\right)\right| \leq \frac{\alpha}{1-\alpha} \frac{z_{2}^{N}}{z_{1}^{N}} \leq\left|\psi_{+}^{\prime}\left(z_{1}^{N}\right)\right|$,

where $\psi_{-}^{\prime}$ and $\psi_{+}^{\prime}$ are the left- resp. righthand side derivatives of $\psi$. Let $x \rightarrow \bar{x}$ as $\Delta \rightarrow 0$. Since $\psi$ is continuous on $[0,1]$, we have that $\bar{x}_{2}=\psi\left(\bar{x}_{1}\right)$. Furthermore, since $\psi$ is decreasing and concave, we have

$$
\left|\psi_{-}^{\prime}\left(\bar{x}_{1}\right)\right| \leq \lim _{\Delta \rightarrow 0}\left|\frac{\psi\left(x_{1}\right)-\psi\left(\frac{\delta_{1}^{\Delta}}{\mu_{1}} x_{1}\right)}{x_{1}-\frac{\delta_{1}^{\Delta}}{\mu_{1}} x_{1}}\right| \leq\left|\psi_{+}^{\prime}\left(\bar{x}_{1}\right)\right|,
$$


and, using l'Hôpital's rule,

$$
\begin{aligned}
\lim _{\Delta \rightarrow 0}\left|\frac{\psi\left(x_{1}\right)-\psi\left(\frac{\delta_{1}^{\Delta}}{\mu_{1}} x_{1}\right)}{x_{1}-\frac{\delta_{1}^{\Delta}}{\mu_{1}} x_{1}}\right| & =\lim _{\Delta \rightarrow 0}\left|\frac{\left(\frac{\delta_{2}^{\Delta}}{\mu_{2}}-1\right) \psi\left(\frac{\delta_{1}^{\Delta}}{\mu_{1}} x_{1}\right)}{\left(1-\frac{\delta_{1}^{\Delta}}{\mu_{1}}\right) x_{1}}\right| \\
& =\lim _{\Delta \rightarrow 0}\left|\frac{\frac{\left(1+\lambda_{2}\right) \delta_{2}^{\Delta} \log \delta_{2}}{\mu_{2}^{2}} \psi\left(\frac{\delta_{1}^{\delta}}{\mu_{1}} x_{1}\right)+\left(\frac{\delta_{2}^{\Delta}}{\mu_{2}}-1\right) \frac{d \psi\left(\frac{\delta_{1}^{\Delta}}{\mu_{1}} x_{1}\right)}{d \Delta}}{-\frac{\left(1+\lambda_{1}\right) \delta_{1}^{\delta} \log \delta_{1}}{\mu_{1}^{2}} x_{1}+\left(1-\frac{\delta_{1}^{\Delta}}{\mu_{1}}\right) \frac{d x_{1}}{d \Delta}}\right| \\
& =\frac{\left(1+\lambda_{2}\right) \log \delta_{2}}{\left(1+\lambda_{1}\right) \log \delta_{1}} \cdot \frac{\psi\left(\bar{x}_{1}\right)}{\bar{x}_{1}} \\
& =\frac{\alpha}{1-\alpha} \cdot \frac{\bar{x}_{2}}{\bar{x}_{1}} .
\end{aligned}
$$

It follows that $\bar{x}=z^{N}$. 


\section{Chapter 3}

\section{The Kalai-Smorodinsky Bargaining Solution with Loss Aversion}

\subsection{Introduction}

In his seminal paper, Nash (1950a) defined the 'bargaining problem' as a proxy for real life bargaining situations. His model is described by two players attempting to find agreement on a certain feasible outcome. They are motivated by the fact that failure to cooperate results in an unfavorable outcome for all. Nash himself defined and characterized a solution for this problem, the well known Nash bargaining solution. A wide range of other solutions have been formulated since. One of the most prominent alternatives to the Nash bargaining solution is the Kalai-Smorodinsky bargaining solution, defined by Raiffa (1953) and characterized by Kalai and Smorodinsky (1975).

In economics - and bargaining is no exception - risk attitudes tend to play a pervasive role on how agents behave. In the bargaining literature, much attention has been paid to the influence of risk attitudes, and in particular risk aversion, on the outcomes players finally agree on according to certain bargaining solutions. Several studies, among which Kannai (1977), Kihlstrom et al. (1981), and Roth (1985), find that the Nash bargaining solution favors the less risk averse bargainer. Kihlstrom et al. (1981) find similar results for the Kalai-Smorodinsky bargaining solution. Köbberling and Peters (2003) also study the effect of risk aversion on the Kalai-Smorodinsky 
solution, but distinguish between probabilistic risk aversion and utility risk aversion. They find it is an advantage to have a more utility risk averse opponent, or a less probabilistically risk averse opponent.

In this chapter we investigate the Kalai-Smorodinsky bargaining solution under a related behavioral phenomenon, named loss aversion. Loss aversion was introduced by Kahneman and Tversky $(1979,1984)$. It is based on the premise that economic agents derive utility from changes in wealth in comparison with some reference point, and that changes for the worse i.e. losses - carry more weight than equivalent changes for the better.

To introduce this concept in the bargaining problem, we follow the approach of Shalev (2002). Each bargainer's preference is represented by a von Neumann-Morgenstern utility function, a nonnegative loss aversion coefficient, and a reference point; if a player's utility level is below his reference point, then he experiences a disutility equal to the size of his incurred loss, inflated by the loss aversion coefficient. Thus, in a bargaining context, incorporating loss aversion of the players is equivalent to applying a particular transformation to the bargaining problem. Reference points are subsequently endogenized by imposing the self-supporting condition. An outcome $z$ is said to be self-supporting under a given solution, whenever transforming the bargaining game using outcome $z$ as reference point, yields a game of which the solution is $z$. We may interpret a bargainer's reference point as the expectation or the aspiration of what utility payoff he may realize given a certain bargaining solution. The self-supporting condition then imposes that the bargaining solution assigns to each player exactly the (initially) aspired utility level, and as a consequence, that no losses are incurred.

We first set out to show that $n$-player bargaining games, $n \geq 2$, have exactly one outcome that is self-supporting under the Kalai-Smorodinsky solution ${ }^{1}$. Kalai and Smorodinsky (1975) defined their solution on two-player bargaining games. In view of the result by Roth (1979) that it is not generally defined on all $n$-player bargaining games, we consider the subclass of all $n$-player bargaining games, defined by Peters and Tijs (1984), which excludes the problematic cases. On these games we define a class of asymmetric $n$-person Kalai-Smorodinsky solutions. Consider an outcome in the Pareto set and the line segments respectively connecting that outcome to the disagreement point and to the utopia point. For any two players we may then project these line segments into the plane. Our solution is defined

\footnotetext{
${ }^{1}$ This was already remarked - without proof - by Shalev (2002) for the case of two-player bargaining games.
} 
by the unique outcome in the Pareto set such that for any two players, the slopes of these projected line segments satisfy a given proportion. We establish that for each loss aversion profile, there is a single bargaining solution in our class that yields the associated self-supporting outcome. This implies uniqueness of a self-supporting outcome under the Kalai-Smorodinsky solution for $n$-player bargaining games. Moreover, the bargaining solution we define provides an easy method of finding this outcome.

We next provide an axiomatic characterization of this class of bargaining solutions. In particular, we show that it is fully defined by the standard properties Strong Individual Rationality, Scale Invariance, and Individual Monotonicity, and a new axiom named Proportional Concession Invariance [PCI]. The axiom of PCI says that if players make concessions with respect to their utopia values in such a way that the new utopia point is on the line segment connecting the solution outcome and the original utopia point, then the solution outcome is left unchanged.

This chapter proceeds as follows. Section 3.2 introduces the notation, and in Section 3.3 we show how loss aversion is incorporated in the bargaining problem. Section 3.4 describes the concept of monotonic curves, their associated bargaining solutions, and defines the Kalai-Smorodinsky bargaining solution as a special case. In Section 3.5 we define a new class of bargaining solutions, and we show that every solution in this class is the self-supporting outcome under the Kalai-Smorodinsky bargaining solution for some loss aversion profile. Section 3.6 contains the axiomatic characterization of this class of bargaining solutions. We conclude in Section 3.7.

Proofs of all claims made in the text are in Appendix 3.A.

\subsection{Preliminaries}

The set of players or bargainers is denoted $N:=\{1, \ldots, n\}$, with $n \geq 2$. For $x, y \in \mathbb{R}^{N}$ we say $x \geq y$ if $x_{i} \geq y_{i}$ for all $i \in N$, and $x>y$ if $x_{i}>y_{i}$ for all $i \in N$. The relations ' $\leq$ ' and ' $<$ ' are defined similarly. We define $\mathbb{R}_{+}^{N}:=\left\{z \in \mathbb{R}^{N} \mid\right.$ $z \geq 0\}$ and $\mathbb{R}_{++}^{N}:=\left\{z \in \mathbb{R}^{N} \mid z>0\right\}$. For $x, y \in \mathbb{R}^{N}$ we denote by $x y$ the vector $\left(x_{1} y_{1}, \ldots, x_{n} y_{n}\right)$, and for $S \subseteq \mathbb{R}^{N}$ we define $x S:=\left\{x y \in \mathbb{R}^{N} \mid y \in S\right\}$. Similarly, we denote $\left(x_{1}+y_{1}, \ldots, x_{n}+y_{n}\right)$ as $x+y$, and the set $\{x+z \mid z \in S\}$ as $x+S$. The vector in $\mathbb{R}^{N}$ that has $i$-th coordinate equal to 1 and all other coordinates equal to 0 is denoted $e^{i}$. The vector $e^{M}, M \subseteq N$ and $M \neq \varnothing$, has all coordinates $i \in M$ equal to 1 , and all coordinates $i \notin M$ equal to 0 . For $x \in \mathbb{R}_{++}^{N}$, we denote $\left(\frac{1}{x_{1}}, \ldots, \frac{1}{x_{n}}\right)$ as $x^{-1}$. 
A bargaining problem for $N$ is defined as a pair $(S, d)$ where

- $S \subset \mathbb{R}^{N}$ is non-empty, closed, convex, and comprehensive,

- $d \in S$,

- there exists a $z \in S$ such that $z>d$, and

- $S_{d}:=\{z \in S \mid z \geq d\}$ is bounded.

A set $S$ is comprehensive if $x \in S$ and $x \geq y$ together imply $y \in S$. In a bargaining context, this assumption can be interpreted as free disposal of utility in the sense that any player can choose a lower utility payoff without it leading to an infeasible outcome. Bargainers seek agreement on an outcome $z$ in $S$ where each player $i \in N$ obtains utility $z_{i}$. In case no agreement is reached the disagreement outcome $d$ results.

The set of all bargaining problems is denoted $B^{N}$. For $(S, d) \in B^{N}$ and each $i \in N$, we define

$$
u_{i}(S, d):=\max \left\{z_{i} \mid z \in S_{d}\right\}
$$

This represents the highest possible utility payoff bargainer $i$ can attain in the bargaining problem $(S, d)$, given that no bargainer $j \in N, j \neq i$, obtains a utility payoff lower than $d_{j}$. The vector $u(S, d):=\left(u_{1}(S, d), \ldots, u_{n}(S, d)\right)$ is termed the utopia point of $(S, d)$. For all $(S, d) \in B^{N}$ we define the Pareto set of $(S, d)$ as

$$
P(S):=\left\{z \in S \mid \text { for all } x \in \mathbb{R}^{N} \text { if } x \geq z \text { and } x \neq z \text {, then } x \notin S\right\} .
$$

A bargaining solution or in short, a solution, is a map $\varphi: B^{N} \rightarrow \mathbb{R}^{N}$ that assigns to any bargaining problem $(S, d) \in B^{N}$ a single point $\varphi(S, d) \in S$.

\subsection{Bargaining with Loss Aversion}

Shalev (2002) introduced a transformation that models loss aversion. Each bargainer $i \in N$ has a non-negative loss aversion coefficient $\lambda_{i}$ and a reference point $r_{i}$. We denote the vector $\left(\lambda_{1}, \ldots, \lambda_{n}\right) \in \mathbb{R}_{+}^{N}$ by $\lambda$. Similarly, $r:=\left(r_{1}, \ldots, r_{n}\right) \in \mathbb{R}^{N}$. Each bargainer $i$ evaluates a utility payoff $z_{i} \in \mathbb{R}$ by the transformation $w_{i}$, defined as

$$
w_{i}\left(z_{i}, \lambda_{i}, r_{i}\right):= \begin{cases}z_{i} & \text { if } z_{i} \geq r_{i} \\ z_{i}-\lambda_{i}\left(r_{i}-z_{i}\right) & \text { if } z_{i}<r_{i}\end{cases}
$$


Thus, a bargainer $i$ who incurs a loss - i.e. obtains a utility payoff $z_{i}$ below his reference point $r_{i}$, experiences a disutility that is equal to his loss $r_{i}-z_{i}$, inflated by the loss aversion coefficient $\lambda_{i}$. Payoffs above the reference point are left unchanged. Note that we may also write

$$
w_{i}\left(z_{i}, \lambda_{i}, r_{i}\right)=\left(1+\lambda_{i}\right) z_{i}-\lambda_{i} \max \left\{r_{i}, z_{i}\right\}
$$

For utility outcomes $z \in \mathbb{R}^{N}$ we define

$$
w(z, \lambda, r):=\left(w_{1}\left(z_{1}, \lambda_{1}, r_{1}\right), \ldots, w_{n}\left(z_{n}, \lambda_{n}, r_{n}\right)\right) .
$$

For sets $T \subset \mathbb{R}^{N}$ we write $w(T, \lambda, r):=\{w(z, \lambda, r) \mid z \in T\}$. Henceforth, the transformation $w: \mathbb{R}^{N} \times \mathbb{R}_{+}^{N} \times \mathbb{R}^{N} \rightarrow \mathbb{R}^{N}$ is referred to as the Shalev transformation.

For bargaining problems $(S, d) \in B^{N}$ we define

$$
w((S, d), \lambda, r):=(w(S, \lambda, r), w(d, \lambda, r)) .
$$

We then have the following.

Lemma 3.3.1. Let $(S, d) \in B^{N}, \lambda \in \mathbb{R}_{+}^{N}$, and $r \in \mathbb{R}^{N}$. Then $w((S, d), \lambda, r) \in B^{N}$.

Let $\varphi: B^{N} \rightarrow \mathbb{R}^{N}$ be some bargaining solution, and let $(S, d)$ be some problem in $B^{N}$. Then by Lemma 3.3.1, $\varphi(w((S, d), \lambda, r))$ is well-defined for all $\lambda \in \mathbb{R}_{+}^{N}$ and $r \in \mathbb{R}^{N}$.

A point $z \in S$ is called a self-supporting outcome under $\varphi$ if

$$
z=\varphi(w((S, d), \lambda, z)) .
$$

Each bargainer $i$ is assumed to have some aspiration or expectation $z_{i}$ about the utility payoff he can realize given the bargaining problem $(S, d)$ and the bargaining solution $\varphi$. Since bargainers are in varying degrees loss averse, and have complete knowledge about their opponents' loss aversion, they do not actually bargain over the given problem $(S, d)$, but rather over the transformed problem $w((S, d), \lambda, z)$, where $z \in \mathbb{R}^{N}$ is the vector of players' expectation levels. Imposing the condition of self-support is then equivalent to imposing that the utility payoffs players realize ex post are consistent with their ex ante expectations. The set of all such points is defined as

$$
\operatorname{Self}^{\varphi}((S, d), \lambda):=\{z \in S \mid z=\varphi(w((S, d), \lambda, z))\} .
$$


Then $\operatorname{Self}^{\varphi}: B^{N} \times \mathbb{R}_{+}^{N} \rightarrow \mathbb{R}^{N}$ is a correspondence that assigns to each bargaining problem $(S, d) \in B^{N}$ and loss aversion profile $\lambda \geq 0$ a possibly empty subset of $S$.

For two-player bargaining games $(S, d)$, Shalev (2002) characterized the set of self-supporting outcomes under the Nash bargaining solution. Specifically, he showed that it is a closed, connected subset of the Pareto set $P(S)$.

\subsection{The Kalai-Smorodinsky Bargaining Solution}

Raiffa (1953), and Kalai and Smorodinsky (1975) defined and characterized a solution - the Kalai-Smorodinsky solution (KS) - for bargaining problems in $B^{\{1,2\}}$. It was shown by Roth (1979) that the $n$-player extension of the KS solution, $n>2$, is not defined on all bargaining problems in $B^{N}$. Therefore, Peters and Tijs (1984) introduced the rather large subclass $I^{N}$ of bargaining problems in $B^{N}$ for which this non-existence result does not hold. Consider the following condition.

For all $x \in S, x \geq d$, and $i \in N$ we have:

$x \notin P(S)$ and $x_{i}<u_{i}(S, d) \Rightarrow$ there is an $\varepsilon>0$ such that $x+\varepsilon e^{i} \in S$

Then

$$
I^{N}:=\left\{(S, d) \in B^{N} \mid(S, d)\right. \text { satifies condition (3.1)\}. }
$$

Condition (3.1) says that if a feasible outcome $x$ is not Pareto optimal, then for any bargainer who does not obtain his utopia payoff there is a feasible outcome that this bargainer strictly prefers over $x$. The class of bargaining problems $(S, 0) \in I^{N}$ is denoted $I_{0}^{N}$; for bargaining problems in $I_{0}^{N}$ we henceforth omit the disagreement point, i.e. we denote $(S, 0) \in I_{0}^{N}$ as $S$.

Peters and Tijs (1984) defined the $n$-player extension of the KS solution by making use of monotonic curves. Specifically, a monotonic curve for $N$ is a map

$$
\vartheta:[1, n] \rightarrow\left\{x \in \mathbb{R}_{+}^{N} \mid x_{i} \leq 1 \text { for all } i \in N, \text { and } 1 \leq \sum_{i \in N} x_{i}\right\},
$$

such that for all $1 \leq s \leq t \leq n$ we have $\vartheta(s) \leq \vartheta(t)$ and $\sum_{i \in N} \vartheta_{i}(s)=s$. The set of all monotonic curves for $N$ is denoted $\Theta^{N}$. 
Lemma 3.4.1 (Peters and Tijs 1984). For each $\vartheta \in \Theta^{N}$ and $S \in I_{0}^{N}$ with $u(S)=e^{N}$, the set

$$
P(S) \cap\{\vartheta(t) \mid t \in[1, n]\}
$$

contains exactly one point.

Let $\vartheta$ be some monotonic curve in $\Theta^{N}$. In view of Lemma 3.4.1 we can define $\rho^{\vartheta}: I^{N} \rightarrow \mathbb{R}^{N}$, the bargaining solution associated with $\vartheta$. Let $S \in I_{0}^{N}$; if $u(S)=e^{N}$, then

$$
\left\{\rho^{\vartheta}(S)\right\}:=P(S) \cap\{\vartheta(t) \mid t \in[1, n]\},
$$

and if $u(S)=\beta$, then $\rho^{\vartheta}(S):=\beta \rho^{\vartheta}\left(\beta^{-1} S\right)$. For $(S, d) \in I^{N}$, we define $\rho^{\vartheta}(S, d):=$ $d+\rho^{\vartheta}(S-d)$. The class of all solutions associated with a monotonic curve in $\Theta^{N}$ is referred to as the class of individually monotonic bargaining solutions. The KS solution is an element of this class. Specifically, it is defined as

$$
\rho^{\hat{\vartheta}} \text { where } \hat{\vartheta}(t):=\frac{t e^{N}}{n} \text { for } t \in[1, n] .
$$

Observe that $\hat{v}$ defines a straight line in $\mathbb{R}^{N}$, which for bargaining games $S \in$ $I_{0}^{N}$ where $u(S)=e^{N}$, is concurrent with the line connecting the disagreement point 0 and the utopia point $e^{N}$. For general bargaining problems $(S, d) \in I^{N}$, the KS solution is the intersection of the Pareto set $P(S)$ and the straight line that connects the disagreement point $d$ and the utopia point $u(S, d)$. To ease notation, we also refer to $\rho^{\hat{\vartheta}}$ as $K S$, i.e. $K S \equiv \rho^{\hat{\vartheta}}$.

\subsection{The Solution Class $\mathscr{D}^{N}$}

We now show that self-supporting outcomes under the KS solution are welldefined, and that each game in $I^{N}$ has exactly one such outcome. Peters and Tijs (1984) show that $I^{\{1,2\}}=B^{\{1,2\}}$, which implies that our result generalizes the remark of Shalev (2002) about the uniqueness of a self-supporting outcome under the KS solution for two-player bargaining games. Furthermore, we introduce a class $\mathscr{D}^{N}$ of bargaining solutions on $I^{N}$, where for any $\lambda \in \mathbb{R}_{+}^{N}$ there is a single $\varphi \in \mathscr{D}^{N}$ such that $\varphi(S, d)$ is the unique self-supporting outcome of the game $(S, d)$ under the KS solution.

Observe that from Lemma 3.3.1 and the fact that the Shalev transformation preserves the ordering of payoffs, we obtain that $(S, d) \in I^{N}$ implies 
$w((S, d), \lambda, r) \in I^{N}$ for all $\lambda \in \mathbb{R}_{+}^{N}$ and $r \in \mathbb{R}^{N}$. Therefore, $\operatorname{Self}^{K S}((S, d), \lambda)$, the set of self-supporting outcomes under the KS solution, is well-defined.

We now introduce the class $\mathscr{D}^{N}$ of bargaining solutions. Denote by $\bar{N}$ the player set without player $n-$ i.e. $\bar{N}:=N \backslash\{n\}$, and define the correspondence $D^{k}: I^{N} \rightarrow \mathbb{R}^{N}$ for all $k \in \mathbb{R}_{++}^{\bar{N}}$ as

$$
\begin{aligned}
& D^{k}(S, d):=\{z \in P(S) \mid \text { for all } i \in \bar{N} \text { we have: } \\
&\left.\left(u_{n}(S, d)-z_{n}\right)\left(z_{i}-d_{i}\right)=k_{i}\left(u_{i}(S, d)-z_{i}\right)\left(z_{n}-d_{n}\right)\right\} .
\end{aligned}
$$

It is not hard to verify that $D^{k} \neq D^{k^{\prime}}$ whenever $k, k^{\prime} \in \mathbb{R}_{++}^{\bar{N}}$ with $k \neq k^{\prime}$. The set $\mathscr{D}^{N}:=\left\{D^{k} \mid k \in \mathbb{R}_{++}^{\bar{N}}\right\}$ is defined as the set that contains all such correspondences. For any $k \in \mathbb{R}_{++}^{\bar{N}}$, define

$$
\vartheta^{k}(t):=\left\{z \in G^{k} \mid \sum_{i=1}^{n} z_{i}=t\right\}
$$

where $t \in[1, n]$, and $G^{k}:=\left\{z \in \mathbb{R}_{+}^{N} \mid\left(1-z_{n}\right) z_{i}=k_{i}\left(1-z_{i}\right) z_{n}\right.$ for all $\left.i \in \bar{N}\right\}$. In Lemma 3.A.1, which can be found in the Appendix, we show that any correspondence $\vartheta^{k}$ is a monotonic curve in $\Theta^{N}$.

Theorem 3.5.1. For all $k \in \mathbb{R}_{++}^{\bar{N}}$ and $(S, d) \in I^{N}$, we have

$$
D^{k}(S, d)=\left\{\rho^{\vartheta^{k}}(S, d)\right\}
$$

It follows from Theorem 3.5.1 that the set $\mathscr{D}^{N}:=\left\{D^{k} \mid k \in \mathbb{R}_{++}^{\bar{N}}\right\}$ is a subset of the class of individually monotonic bargaining solutions. The following theorem establishes the equivalence between $\left\{\operatorname{Self}^{K S}(., \lambda) \mid \lambda \in \mathbb{R}_{+}^{N}\right\}$ and $\mathscr{D}^{N}$.

Theorem 3.5.2. For all $(S, d) \in I^{N}$ we have

$$
\operatorname{Self}^{K S}((S, d), \lambda)=D^{k}(S, d)
$$

where $k:=\left(k_{1}, \ldots, k_{n-1}\right)$, and $k_{i}:=\frac{1+\lambda_{n}}{1+\lambda_{i}}$ for all $i \in \bar{N}$. That is,

$\operatorname{Self}^{K S}((S, d), \lambda):=\{z \in P(S) \mid$ for all $i \in \bar{N}$ we have:

$$
\left.\left(1+\lambda_{i}\right)\left(u_{n}(S, d)-z_{n}\right)\left(z_{i}-d_{i}\right)=\left(1+\lambda_{n}\right)\left(u_{i}(S, d)-z_{i}\right)\left(z_{n}-d_{n}\right)\right\}
$$


With a slight abuse of notation, we henceforth consider $D^{k}(S, d)$ to be an outcome. That is, if $D^{k}(S, d)=\{z\}$, then we write $D^{k}(S, d)=z$. Then also

$$
D^{k}(S, d)=\left(D_{1}^{k}(S, d), \ldots, D_{n}^{k}(S, d)\right) .
$$

From Theorem 3.5.2 it follows that for each loss aversion profile $\lambda \in \mathbb{R}_{+}^{N}$, we may look at $\operatorname{Self}^{K S}(., \lambda)$ as an asymmetric $n$-player Kalai-Smorodinsky solution where the asymmetry is fully defined by the bargainers' degrees of loss aversion. In the following section we provide an axiomatic characterization of these solutions.

\subsection{An Axiomatic Characterization of $\mathscr{D}^{N}$}

From Theorem 3.5.1 it follows that $\mathscr{D}^{N}$ is a subclass of the individual monotonic bargaining solutions, defined and characterized by Peters and Tijs (1984). Of their axioms we retain Scale Invariance [SI] and Individual Monotonicity [IM].

(SI) $\varphi: B^{N} \rightarrow \mathbb{R}^{N}$ satisfies Scale Invariance if $t(\varphi(S, d))=\varphi(t(S), t(d))$, where $t: \mathbb{R}^{N} \rightarrow \mathbb{R}^{N}$ is a linear transformation $t(x):=\alpha+\beta x$, with $\alpha \in \mathbb{R}^{N}$, $\beta \in \mathbb{R}_{++}^{N}$, and $t(S):=\alpha+\beta S$ for $S \subset \mathbb{R}^{N}$.

(IM) $\varphi: B^{N} \rightarrow \mathbb{R}^{N}$ satisfies Individual Monotonicity if $\varphi_{i}(S, d) \leq \varphi_{i}(T, d)$ for all $(S, d),(T, d) \in B^{N}$ and $i \in N$ with $S \subseteq T$ and $u_{j}(S)=u_{j}(T)$ for all $j \in N \backslash\{i\}$.

The axiom of SI is consistent with the premise that bargainers' preferences are representable by von Neumann-Morgenstern utility functions. Kalai and Smorodinsky (1975) introduced IM as a possible alternative to Independence of Irrelevant Alternatives, defined by Nash (1950a), after this axiom had been criticized. A third well-known property we require is Strong Individual Rationality [SIR].

(SIR) $\varphi: B^{N} \rightarrow \mathbb{R}^{N}$ satisfies Strong Individual Rationality if $\varphi(S, d)>d$ for all $(S, d) \in B^{N}$.

We introduce a novel axiom called Proportional Concession Invariance [PCI]. One can consider a solution outcome $\varphi(S, d)$ as a representation of concessions that bargainers make with respect to their utopia point values 
$u(S, d)$. The PCI axiom then says that if we replace $u(S, d)$ by a point $\hat{u}$ on the line segment connecting $\varphi(S, d)$ and $u(S, d)$, and shrink the bargaining set accordingly, then the solution outcome should not change. Put differently, if the players' utopia values are reduced in such a way that their concessions with respect to the original solution outcome change proportionally, then this solution outcome should not change.

(PCI) $\varphi: B^{N} \rightarrow \mathbb{R}^{N}$ satisfies Proportional Concession Invariance if for a bargaining problem $(S, d) \in B^{N}$ with solution $\varphi(S, d)$, and a bargaining problem $(\hat{S}, d)$ with

$$
\hat{S}:=\{z \in S \mid z \leq \hat{u}\},
$$

where $\hat{u}=\alpha \varphi(S, d)+(1-\alpha) u(S, d)$ for some $\alpha \in[0,1]$, we have $\varphi(\hat{S}, d)=$ $\varphi(S, d)$.
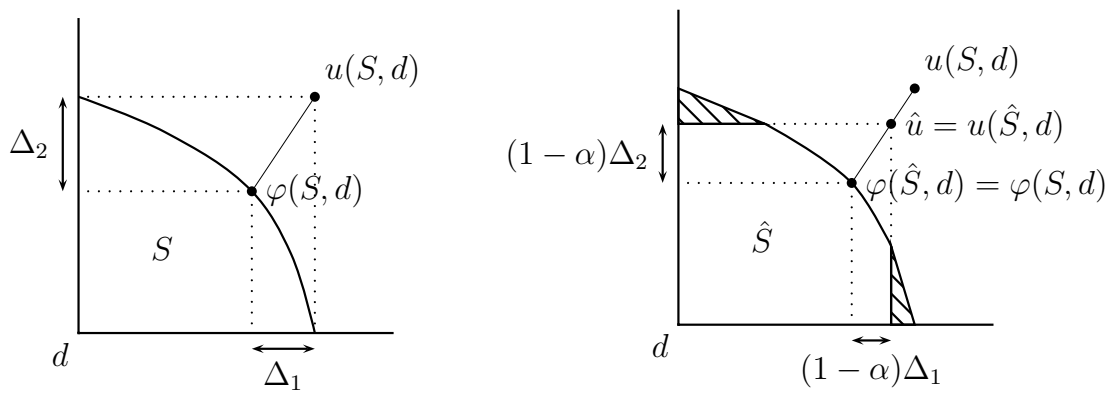

Figure 3.1: A visual representation of PCI in two-player bargaining games.

The property PCI can also be thought of as a very weak form of IIA, and is therefore satisfied by the Nash bargaining solution and its asymmetric variants. Furthermore, observe that PCI is the counterpart to Disagreement Point Convexity [DPC], one of the axioms used by Peters and van Damme (1991) to characterize the class of asymmetric Nash bargaining solutions. This property requires that the solution outcome $\varphi(S, d)$ remain unchanged if we replace $d$ by a point $\hat{d}$ on the line segment connecting the disagreement point $d$ and the solution outcome $\varphi(S, d)$.

It is worth noting that the characterization of $\mathscr{D}^{N}$ makes no use of Pareto Optimality [PO]. In fact, in the Appendix, we use an argument similar as in Roth (1977) to show that PO is implied by our axioms. The following theorem states our characterization result. 
Theorem 3.6.1. Let $\varphi: I^{N} \rightarrow \mathbb{R}^{N}$ be a bargaining solution. Then $\varphi \in \mathscr{D}^{N}$ if and only if $\varphi$ satisfies SIR, SI, IM, and PCI.

\subsection{Concluding Remarks}

It was established in this chapter that bargaining games with $n$ possibly loss averse players, $n \geq 2$, have exactly one self-supporting outcome under the Kalai-Smorodinsky bargaining solution. Moreover, an asymmetric $n$ player version of the KS solution was defined which directly captures the asymmetry resulting from the bargainers' degrees of loss aversion. To be more precise, we consider the symmetric $n$-player version of the KS solution, and - following the method of Shalev - we correct for the players' differences in bargaining power that stem from the varying extents to which they are loss averse. It was shown that for any loss aversion profile, the outcome thus obtained is given by exactly one bargaining solution in the class $\mathscr{D}^{N}$ of asymmetric $n$-player KS solutions. These results are summarized in the following corollary.

Corollary 3.7.1. Let $\lambda \in \mathbb{R}_{+}^{N}$ be the profile of loss aversion. For every $(S, d) \in$ $I^{N}$, an outcome $z \in S$ is self-supporting under the KS solution if and only if $z=D^{k}(S, d)$, where $k_{i}:=\frac{1+\lambda_{n}}{1+\lambda_{i}}$ for all $i \in \bar{N}$.

We have characterized the class $\mathscr{D}^{N}$ of all bargaining solutions $D^{k}$ by the axioms of Strong Individual Rationality, Scale Invariance, Individual Monotonicity, and Proportional Concession Invariance. While the first three properties are standard in the axiomatic bargining literature, the last one is newly defined in this article.

A careful comparison between the axiomatic characterizations of $\mathrm{KS}$ and $\mathscr{D}^{N}$ reveals that the correction needed to account for bargainers' loss aversion is equivalent to relaxing the axiom of Symmetry, and imposing that the solution remain unchanged when players make concessions with respect to their utopia values in a proportion that is determined, uniquely, by the loss aversion coefficients. 


\section{A Appendix: Proofs}

\section{A.1 The proof of Lemma 3.3.1}

Let $(S, d) \in B^{N}, \lambda \in \mathbb{R}_{+}^{N}$, and $r \in \mathbb{R}^{N}$. Since the Shalev transformation is continuous, one-to-one, and preserves the ordering of payoffs, we have that

- $w(S, \lambda, r)$ is non-empty, closed, and comprehensive,

- $w(d, \lambda, r) \in w(S, \lambda, r)$,

- there are $z \in w(S, \lambda, r)$ with $z>w(d, \lambda, r)$, and

- $w\left(S_{d}, \lambda, r\right)$ is bounded.

Left to show is that $w(S, \lambda, r)$ is convex. Let $x, y \in S$ and $t \in[0,1]$. By convexity of $S$, we have $t x+(1-t) y \in S$. By the fact that $w$ is concave in the first coordinate, we have

$$
w(t x+(1-t) y, \lambda, r) \geq t w(x, \lambda, r)+(1-t) w(y, \lambda, r) .
$$

By comprehensiveness of $w(S, \lambda, r)$ this implies

$$
t w(x, \lambda, r)+(1-t) w(y, \lambda, r) \in w(S, \lambda, r) .
$$

This implies convexity of $w(S, \lambda, r)$.

\section{A.2 The proof of Theorem 3.5.1}

First, we show the following.

Lemma 3.A.1. Let $k \in \mathbb{R}_{++}^{\bar{N}}$. Then the correspondence $\vartheta^{k}(t), t \in[1, n]$, is a monotonic curve.

Proof. Let $\bar{s} \in[1, n]$. We show that there is a unique point $z^{*} \in \mathbb{R}_{+}^{N}$ such that $\vartheta^{k}(\bar{s})=z^{*}$. That is, we show that the system of equations

$$
\begin{aligned}
& \left(1-z_{n}\right) z_{i}=k_{i}\left(1-z_{i}\right) z_{n} \text { for all } i \in \bar{N} \\
& \sum_{j=1}^{n} z_{j}=\bar{s},
\end{aligned}
$$

has exactly one solution $z^{*}$ in $\mathbb{R}_{+}^{N}$. Suppose the system has a solution $z \in \mathbb{R}_{+}^{N}$, and suppose $z_{n}>1$. Then for each $i \in \bar{N}$ we either have $z_{i}>1$ or $z_{i}<0$, 
which by $z \in \mathbb{R}_{+}^{N}$ implies $z_{i}>1$ for all $i \in N$. Since this is a violation of (3.5), we must have $z_{n} \leq 1$. Since $\left(1-k_{i}\right)^{-1} \notin[0,1]$ for all $i \in \bar{N}$, we can write

$$
z_{i}=\frac{k_{i} z_{n}}{1-z_{n}\left(1-k_{i}\right)} \text { for all } i \in \bar{N} \text {. }
$$

Then for all $i \in \bar{N}, z_{i}$ is strictly increasing in $z_{n}$ on the domain [0,1]. The observations that

$$
\begin{array}{ll}
\sum_{j=1}^{n} z_{j}=0 \leq \bar{s} & \text { for } z_{n}=0, \text { and } \\
\sum_{j=1}^{n} z_{j}=n \geq \bar{s} & \text { for } z_{n}=1,
\end{array}
$$

together with continuity of $\sum_{j=1}^{n} z_{j}$ in $z_{n}$ then imply that there is exactly one $z^{*} \in \mathbb{R}_{+}^{N}$ that solves the system of equations (3.4) and (3.5). It follows that the correspondence $\vartheta^{k}$ is a map. Moreover, for all $1 \leq s \leq t \leq n$ we have $0<\vartheta^{k}(s) \leq \vartheta^{k}(t) \leq e^{N}$ and $\sum_{i \in N} \vartheta_{i}^{k}(s)=s$. It follows that $\vartheta^{k}$ is a monotonic curve.

To prove Theorem 3.5.1, we show that each map $D^{k} \in \mathscr{D}^{N}$ is the bargaining solution associated with the monotonic curve $\vartheta^{k}$ as defined in (3.3).

Proof of Theorem 3.5.1. Consider a normalized bargaining problem $T \in$ $I_{0}^{N}$, i.e. $u(T)=e^{N}$. Let $k \in \mathbb{R}_{++}^{\bar{N}}$ and observe that by (3.2) we have

$$
D^{k}(T)=P(T) \cap G^{k} .
$$

By convexity of $T$ we have $P(T) \subset\left\{z \in \mathbb{R}_{+}^{N} \mid \sum_{j \in N} z_{j} \geq 1\right\}$, implying

$$
D^{k}(T)=P(T) \cap\left\{\vartheta^{k}(t) \mid t \in[1, n]\right\}
$$

where $\vartheta^{k}$ is defined by (3.3). It follows that $D^{k}(T)=\left\{\rho^{\vartheta^{k}}(T)\right\}$. From this it is easily established that

$$
D^{k}(S, d)=\left\{\rho^{\vartheta^{k}}(S, d)\right\}
$$

for all $(S, d) \in I^{N}$. 


\section{A.3 The Proof of Theorem 3.5.2}

We start with the following lemma.

Lemma 3.A.2. Let $(S, d) \in I^{N}$. Then $D^{k}(S, d)=K S(S, d)$ if and only if $k=$ $e^{\bar{N}}$.

Proof. It is easy to show that for any $t \in[1, n)$, we have $\vartheta^{k}(t)=\hat{\vartheta}(t)$ if and only if $k=e^{\bar{N}}$. The result then follows from Theorem 3.5.1 and the definition of $K S$.

Proof of Theorem 3.5.2. Let $(S, d) \in I^{N}$ and $\lambda \in \mathbb{R}_{+}^{N}$, and let $u \equiv u(S, d)$. By Lemma 3.A.2 and the fact that $K S(S, d) \geq d$, we have

$$
\begin{aligned}
& K S(w((S, d), \lambda, z))=\{x \in P(w(S, \lambda, z)) \mid \text { for all } i \in \bar{N} \text { we have } \\
& \left.\quad\left(u_{n}-x_{n}\right)\left(x_{i}-\left(1+\lambda_{i}\right) d_{i}+\lambda_{i} z_{i}\right)=\left(u_{i}-x_{i}\right)\left(x_{n}-\left(1+\lambda_{n}\right) d_{n}+\lambda_{n} z_{n}\right)\right\} .
\end{aligned}
$$

Observe that $z \in \operatorname{Self}^{K S}((S, d), \lambda)$ if and only if $z=K S(w((S, d), \lambda, z))$. That is, $z \in \operatorname{Self}^{K S}((S, d), \lambda)$ iff

(i) $z \in P(w(S, \lambda, z))$, and

(ii) $\left(u_{n}-z_{n}\right)\left(z_{i}-\left(1+\lambda_{i}\right) d_{i}+\lambda_{i} z_{i}\right)=\left(u_{i}-z_{i}\right)\left(z_{n}-\left(1+\lambda_{n}\right) d_{n}+\lambda_{n} z_{n}\right)$ for all $i \in \bar{N}$.

From $z=w(z, \lambda, z), P(w(S, \lambda, z))=w(P(S), \lambda, z)$, and the fact that the Shalev transformation is one-to-one, it follows that $z \in P(w(S, \lambda, z))$ is equivalent to $z \in P(S)$.

Define $k:=\left(k_{1}, \ldots, k_{n-1}\right)$ where $k_{i}:=\frac{1+\lambda_{n}}{1+\lambda_{i}}$ for all $i \in \bar{N}$. Observe that the statement in (ii) is equivalent to

$$
\left(u_{n}-z_{n}\right)\left(z_{i}-d_{i}\right)=k_{i}\left(u_{i}-z_{i}\right)\left(z_{n}-d_{n}\right) \text { for all } i \in \bar{N} \text {. }
$$

It follows that $z \in \operatorname{Self}^{K S}((S, d), \lambda)$ is equivalent to

$$
z \in\left\{x \in P(S) \mid\left(u_{n}-x_{n}\right)\left(x_{i}-d_{i}\right)=k_{i}\left(u_{i}-x_{i}\right)\left(x_{n}-d_{n}\right) \text { for all } i \in \bar{N}\right\} .
$$

Hence, $\operatorname{Self}^{K S}((S, d), \lambda)=D^{k}(S, d)$. 


\section{A.4 The proof of Theorem 3.6.1}

The standard axiom of Pareto Optimality is useful for the proof.

(PO) $\varphi: B^{N} \rightarrow \mathbb{R}^{N}$ satisfies PO if $\varphi(S, d) \in P(S)$ for all $(S, d) \in B^{N}$.

From Peters and Tijs (1984) we obtain the following.

Lemma 3.A.3 (Peters and Tijs 1984). Let $\varphi: I^{N} \rightarrow \mathbb{R}^{N}$ be a bargaining solution. Then $\varphi$ satisfies $P O$, SI, and IM, if and only if $\varphi=\rho^{\vartheta}$ for some $\vartheta \in \Theta^{N}$.

This we use to establish the following result.

Proposition 3.A.4. Let $\varphi: I^{N} \rightarrow \mathbb{R}^{N}$ be a bargaining solution in $\mathscr{D}^{N}$. Then $\varphi$ satisfies SIR, SI, IM, and PCI.

Proof. Since $\varphi \in \mathscr{D}^{N}$ we have $\varphi=D^{k}$ for some $k \in \mathbb{R}_{++}^{\bar{N}}$. By Theorem 3.5.1, we have $\varphi=\rho^{\vartheta}$ where $\vartheta \in \Theta^{N}$, which by Lemma 3.A.3 implies that $\varphi$ satisfies SI and IM.

Consider a bargaining problem $(S, d) \in I^{N}$, and the solution $\varphi(S, d)=: z$. To see that $\varphi$ satisfies SIR, suppose there is an $i \in N$ such that $z_{i}=d_{i}$. Observe that $z \in P(S)$ and

$$
\left(u_{n}(S, d)-z_{n}\right)\left(z_{j}-d_{j}\right)=k_{j}\left(u_{j}(S, d)-z_{j}\right)\left(z_{n}-d_{n}\right) \text { for all } j \in \bar{N} .
$$

If $i=n$, then $z_{j}=d_{j}$ for all $j \in \bar{N}$, implying $z=d$. Let $i \in \bar{N}$, and observe that $z_{i}=d_{i}$ implies $z_{n}=d_{n}$, and thus $z=d$. Since $d \notin P(S)$, we arrive at a contradiction. It follows that $z>d$.

To see that $\varphi$ satisfies PCI, consider the problem $(\hat{S}, d) \in I^{N}$ where

$$
\hat{S}:=\{x \in S \mid x \leq \hat{u}\}
$$

with $\hat{u}=\alpha z+(1-\alpha) u(S, d)$ for some $\alpha \in(0,1)$. Then $\hat{u}-z=(1-\alpha)(u(S, d)-z)$, implying that

$$
\left(\hat{u}_{n}-z_{n}\right)\left(z_{i}-d_{i}\right)=k_{i}\left(\hat{u}_{i}-z_{i}\right)\left(z_{n}-d_{n}\right) \text { for all } i \in \bar{N} .
$$

Since $z \in \hat{S}$ and $z \in P(S)$, we have $z \in P(\hat{S})$. This and (3.6) together imply $D^{k}(\hat{S}, d)=z$. Hence, $\varphi$ satisfies PCI.

For the reverse implication we require two additional lemmas. 
Lemma 3.A.5. Let $(S, d) \in I^{N}$, and $z \in S \backslash P(S)$. Then for the function $f$ : $[0,1] \rightarrow \mathbb{R}^{N}$ defined as

$$
f(\alpha):=(1-\alpha) z+\alpha u(S, d),
$$

there is exactly one $\alpha^{*} \in[0,1]$ such that $f\left(\alpha^{*}\right) \in P(S)$.

Proof. By compactness of $S$ we have that

$$
\alpha^{*}:=\max \{\alpha \mid f(\alpha) \in S\}
$$

is well-defined. We now show that $f\left(\alpha^{*}\right) \in P(S)$. Suppose $f\left(\alpha^{*}\right) \notin P(S)$. By condition (3.1), it follows that for each $i \in N$ with $z_{i}<u_{i}(S, d)$, there is an $\varepsilon_{i}>0$ such that $f\left(\alpha^{*}\right)+\varepsilon_{i} e^{i} \in S$. Then by convexity of $S$ there is an $\varepsilon>0$, such that

$$
f\left(\alpha^{*}\right)+\varepsilon(u(S, d)-z) \in S .
$$

But then there is a $\beta>\alpha^{*}$ with $f(\beta) \in S$. This is a contradiction.

To show uniqueness, let $\alpha_{1}, \alpha_{2} \in[0,1]$ with $\alpha_{1} \neq \alpha_{2}$, and suppose $f\left(\alpha_{1}\right), f\left(\alpha_{2}\right) \in$ $P(S)$. Without loss of generality, assume $\alpha_{2}>\alpha_{1}$. Then since $u(S, d) \geq z$ and $u(S, d) \neq z$, we have $f\left(\alpha_{2}\right) \geq f\left(\alpha_{1}\right)$ and $f\left(\alpha_{2}\right) \neq f\left(\alpha_{1}\right)$. Since $f\left(\alpha_{1}\right) \in P(S)$, this implies $f\left(\alpha_{2}\right) \notin S$, a contradiction.

Lemma 3.A.6. Let $\varphi: I^{N} \rightarrow \mathbb{R}^{N}$ be a solution satisfying SIR, SI, and PCI. Then $\varphi$ satisfies $P O$.

Proof. Let $\varphi: I^{N} \rightarrow \mathbb{R}^{N}$ be a bargaining solution satisfying SIR, SI, and PCI. By SI it is sufficient to restrict attention to bargaining problems in $I_{0}^{N}$. Let $S \in I_{0}^{N}$. By SIR we have $\varphi(S)>0$. Now assume $\varphi(S) \notin P(S)$. By Lemma 3.A.5, there is a single $z^{*} \in P(S)$, such that

$$
z^{*}=(1-\alpha) \varphi(S)+\alpha u(S) \text { for some } \alpha \in(0,1] .
$$

Define $\hat{S}:=\left\{x \in S \mid x \leq z^{*}\right\}$, and observe that by PCI we have

$$
\varphi(\hat{S})=\varphi(S) .
$$

Similarly, for the set $T:=\{x \in S \mid x \leq \varphi(S)\}$ we have $\varphi(T)=\varphi(S)$. Now observe that $\hat{S}=\left[z^{*}(\varphi(S))^{-1}\right] T$. Then by SI we have

$$
\varphi(\hat{S})=z^{*}(\varphi(S))^{-1} \varphi(T)=z^{*}(\varphi(S))^{-1} \varphi(S)=z^{*} .
$$

This contradicts $\varphi(\hat{S})=\varphi(S)$. 
Proposition 3.A.7. Let $\varphi: I^{N} \rightarrow \mathbb{R}^{N}$ be a bargaining solution satisfying $S I R$, SI, IM, and PCI. Then $\varphi \in \mathscr{D}^{N}$.

Proof. Since $\varphi$ satisfies SIR, SI, and PCI, it follows from Lemma 3.A.6 that $\varphi$ satisfies PO. Then by Lemma 3.A.3 it follows that $\varphi \equiv \rho^{\vartheta^{*}}$ for some monotonic curve $\vartheta^{*} \in \Theta^{N}$.

Consider the problem

$$
H:=\operatorname{Conv}\left(\left\{e^{i} \mid i \in N\right\} \cup\{0\}\right),
$$

and observe that $H \in I_{0}^{N}$. If there is an $i \in N$ with $\varphi_{i}(H)=1$, then $\varphi_{j}(H)=0$ for all $j \neq i$, which is a violation of SIR. Hence, $0<\varphi(H)<e^{N}$. It follows that $k:=\left(k_{1}, \ldots, k_{n-1}\right)$, where

$$
k_{i}:=\frac{1-\varphi_{n}(H)}{1-\varphi_{i}(H)} \cdot \frac{\varphi_{i}(H)}{\varphi_{n}(H)}
$$

is well-defined and $k \in \mathbb{R}_{++}^{\bar{N}}$.

In what follows, we show that

$$
\vartheta^{*}(t)=\vartheta^{k}(t)
$$

for all $1 \leq t \leq n$. We do this in three steps. Let $\bar{t} \in[1, n]$. Then

1. we construct a specific problem $S \in I_{0}^{N}$,

2. we show that $\varphi(S)=\vartheta^{k}(\bar{t})$, and

3. we show that $\varphi(S)=\vartheta^{*}(\bar{t})$.

By (3.8) we have $\rho^{\vartheta^{k}}=\rho^{\vartheta^{*}}$, which together with $\varphi=\rho^{\vartheta^{*}}$ and $D^{k}=\rho^{\vartheta^{k}}$ establishes $\varphi=D^{k}$.

Step 1: Define the function $g:[0,1] \rightarrow[1, n]$ as

$$
g(\alpha):=\sum_{i \in N} \frac{\varphi_{i}(H)}{\alpha \varphi_{i}(H)+(1-\alpha)} .
$$

From the fact that $g$ is strictly increasing ${ }^{2}$ and continuous, and the fact that $g(0)=1$ and $g(1)=n$, it follows that for each $t \in[1, n]$ there is a unique $\alpha \in[0,1]$ such that $g(\alpha)=t$.

\footnotetext{
${ }^{2}$ This follows from $\varphi(H) \neq 0$ and $\varphi(H) \neq e^{N}$.
} 
Let $\beta:=\bar{\alpha} \varphi(H)+(1-\bar{\alpha}) e^{N}$, where $\bar{\alpha}$ is such that $g(\bar{\alpha})=\bar{t}$. Then define the problem $S \in I_{0}^{N}$ as

$$
S:=\left\{\beta^{-1} z \mid z \in H \text { and } z \leq \beta\right\} .
$$

Since $\varphi$ satisfies SI and PCI, we have

$$
\varphi(S)=\beta^{-1} \varphi(H) .
$$

It follows from (3.10) and (3.9), and the fact that $g(\bar{\alpha})=\bar{t}$ that $\sum_{i \in N} \varphi_{i}(S)=\bar{t}$.

Step 2: Rewriting (3.7) yields

$$
\left(1-\varphi_{n}(H)\right) \varphi_{i}(H)=k_{i}\left(1-\varphi_{i}(H)\right) \varphi_{n}(H) \text { for all } i \in \bar{N} .
$$

From the definition of $\beta$ we have $\beta-\varphi(H)=(1-\bar{\alpha})\left(e^{N}-\varphi(H)\right)$. Thus,

$$
\left(\beta_{n}-\varphi_{n}(H)\right) \varphi_{i}(H)=k_{i}\left(\beta_{i}-\varphi_{i}(H)\right) \varphi_{n}(H) \text { for all } i \in \bar{N} .
$$

For each $i \in \bar{N}$, we can multiply both sides of the equation by $\frac{1}{\beta_{n} \beta_{i}}$. By (3.10) this yields

$$
\left(1-\varphi_{n}(S)\right) \varphi_{i}(S)=k_{i}\left(1-\varphi_{i}(S)\right) \varphi_{n}(S) \text { for all } i \in \bar{N} .
$$

It follows that $\varphi(S) \in G^{k}$. Since $\sum_{i \in N} \varphi_{i}(S)=\bar{t}$, we have

$$
\varphi(S)=\vartheta^{k}(\bar{t})
$$

Step 3: Since $\varphi \equiv \rho^{\vartheta^{*}}$, and $S \in I_{0}^{N}$ with $u(S)=e^{N}$, we have

$$
\{\varphi(S)\}=P(S) \cap\left\{\vartheta^{*}(t) \mid t \in[1, n]\right\},
$$

which implies $\varphi(S)=\vartheta^{*}\left(t^{*}\right)$ for some $t^{*} \in[1, n]$. From the definition of monotonic curves we obtain

$$
t^{*}=\sum_{i \in N} \vartheta_{i}^{*}\left(t^{*}\right)=\sum_{i \in N} \varphi_{i}(S)=\bar{t} .
$$

Hence,

$$
\varphi(S)=\vartheta^{*}(\bar{t}) .
$$

Combining (3.11) and (3.12) yields the desired result.

Theorem 3.6.1 is established by Propositions 3.A.4 and 3.A.7. 


\section{Chapter 4}

\section{On Loss Aversion in Bimatrix Games}

\subsection{Introduction}

Since von Neumann and Morgenstern (1944) developed expected utility theory, it has been the dominant approach in decision making under uncertainty. The pervasive role of expected utility in the economics of uncertainty has also been subject to criticism. One of the earliest examples is the Allais paradox (Allais 1953) ${ }^{1}$.

Several alternative models for decision under uncertainty have been proposed. One of the most prolific examples of these nonexpected utility theories is 'prospect theory', developed by Kahneman and Tversky (1979). In this model it is assumed that economic agents make choices between lotteries in two phases: an editing phase and an evaluation phase. In the editing phase, agents observe and interpret the options between which they must choose using several simple heuristics, one of which is the framing of payoffs as gains or as losses, using a reference point. In the evaluation phase, an agent modifies his utility function to a reference-dependent utility function, to account for the perception of the payoffs. That is, perceived losses are weighted downwards, a phenomenon frequently referred to as loss aversion. The agent then transforms the probabilities with which payoffs are realized by using a probability weighting function, and uses these modified proba-

\footnotetext{
${ }^{1}$ For surveys of the literature on violations of expected utility theory, see Schoemaker (1982) or Machina (1987).
} 
bilities to calculate the expected reference-dependent utility of the lottery. Here, we ignore the latter aspect.

Although expected utility theory remains the most important model for rational decision making in mainstream economic theory, the nonexpected utility theories - and prospect theory in particular - have proved to be successful challengers of the expected utility paradigm. Many of these theories have an equally solid mathematical basis as expected utility theory, making them acceptable alternatives for economists. More importantly, they tend to incorporate a number of behavioral patterns, documented in the psychology literature ${ }^{2}$ that better explain the decisions of economic agents, and as a consequence, are better able to provide a theoretical basis for several empirically observed phenomena that do not fit with the standard theory of rational choice ${ }^{3}$.

Although a number of these behavioral aspects have been applied to the specific field of noncooperative game theory ${ }^{4}$, the effects of loss aversion on non-cooperative games have not been extensively studied. There is some work in which the outcomes of certain well known examples of games are showed to be consistent with experimental or empirical observations, if the players are assumed to be loss averse ${ }^{5}$. However, this literature focuses on specific examples, and furthermore, usually assumes that the players' reference points are given by some exogenous status quo value. This does not fully reflect the idea of reference-dependence as it was originally intended: Tversky and Kahneman (1981) defined the framing of payoffs as 'the decision-maker's conception of the acts, outcomes, and contingencies associated with a particular choice' (p. 453). This implies that the reference points of players playing a noncooperative game should not be fixed ex ante, but must be based on their own strategies (the acts), their payoffs (the outcomes), and the strategies of their opponents (the contingencies). Thus, game theory adds another dimension to the issue of framing payoffs, and to loss aversion in general, that is often ignored.

One paper in which reference-dependence is treated consistently with Tversky and Kahneman's definition is (Shalev 2000). There, an equilibrium concept is developed in which each player transforms his basic utility pay-

\footnotetext{
${ }^{2}$ See for instance Kahneman and Tversky (1979), and Hershey et al. (1982), and others.

${ }^{3} \mathrm{An}$ overview of puzzles and the solutions proposed by prospect theory is provided in Camerer (2002).

${ }^{4}$ Examples are Crawford (1990), Dekel et al. (1991), and Eichberger and Kelsey (1999).

${ }^{5}$ For example, Fershtman (1996) studies an incumbency game, Berejikian (2002) a.o. the game of chicken and the prisoners' dilemma, and Butler (2007) an ultimatum game.
} 
offs with a reference point such that his expected reference-dependent equilibrium payoff is exactly equal to that reference point. Thus, the players' reference points can be interpreted as their expected payoffs in equilibrium. In line with Tversky and Kahneman's definition, the reference points thus depend on the equilibrium strategies and on the players' individual basic utility payoff matrices.

We develop two other equilibrium concepts that take into account the players' loss aversion in a way that is consistent with Kahneman and Tversky's definition. Unlike Shalev, we only consider bimatrix games, i.e., twoplayer games in which each player has a finite number of pure strategies. The first new concept, called 'maximin loss aversion equilibrium', assumes that each player's reference point is equal to his pure maximin value, taking into account only those pure strategies of the opponent that are played with positive probability. This differs from Shalev's equilibrium concept in two significant ways: first, a player's reference point depends on the support of the opponent's strategy. In addition, it assumes that players are cautious, in the sense that they base their reference points on 'worst-case' values. Since a player's reference point depends on nothing but the support of his opponent's strategy, it can exhibit discontinuities when the opponent's support changes. Indeed, maximin loss aversion equilibrium may fail to exist. Nonetheless, we show existence if at least one player has at most two pure strategies.

The loss aversion safety level of a player is the value of the matrix game, derived from the basic payoff matrix with that value as reference point. A safety level loss aversion equilibrium is an equilibrium in the bimatrix game obtained by transforming the basic payoffs with these loss aversion safety levels as reference points. This type of equilibrium shares the fixed point idea with Shalev's loss aversion equilibrium and the 'cautious player' property with the maximin loss aversion equilibrium. However, it is based on reference points that no longer depend on the opponent's equilibrium strategy, and that represent the payoff that a player can guarantee for himself.

We conclude the paper with a comparative statics analysis of the three equilibrium concepts in $2 \times 2$ bimatrix games. Specifically, we assume that both players are loss neutral, but that player 2 believes that player 1 is loss averse. We study the effect this has on the equilibrium payoff of player 1 , and establish under which condition this is beneficial to player 1 .

This chapter continues as follows. After preliminaries in Section 4.2, we discuss Shalev's (2000) 'myopic loss aversion equilibrium' in Section 4.3. Section 4.4 discusses the maximin loss aversion equilibrium, and Section 
4.5 the safety level loss aversion equilibrium. In Section 4.6 we present the comparative statics results mentioned above. Proofs are collected in the Appendix.

\subsection{Preliminaries}

\subsubsection{Bimatrix Games and Nash Equilibrium}

Players 1 and 2 have sets of pure strategies $I=\{1, \ldots, m\}$ and $J=\{1, \ldots, n\}$, respectively. If player 1 plays $i$ and player 2 plays $j$, then player 1 [2] receives $a_{i j}\left[b_{i j}\right]$, the number in the payoff matrix $A[B]$ in row $i$ and column $j$. The pair $(A, B)$ is called a bimatrix game.

The $(k-1)$-dimensional unit simplex $\Delta^{k}$ is defined as

$$
\Delta^{k}:=\left\{\omega \in \mathbb{R}^{k}: \sum_{l=1}^{k} \omega_{l}=1 \text { and } \omega_{i} \geq 0 \text { for all } i=1, \ldots, k\right\} .
$$

A mixed strategy for player 1 [2] is an element of $\Delta^{m}\left[\Delta^{n}\right]$. A pure strategy $l$ is identified with the mixed strategy $e^{l}$, where $e^{l}$ is the vector with a one in position $l$ and zeros otherwise. The support of a player's strategy is the set of pure strategies that a player plays with positive probability. That is,

$$
\mathrm{S}(p):=\left\{i \in I: p_{i}>0\right\} \text { and } \mathrm{S}(q):=\left\{j \in J: q_{j}>0\right\} .
$$

A Nash equilibrium in an $m \times n$ bimatrix game $(A, B)$ is a pair $\left(p^{*}, q^{*}\right) \in$ $\Delta^{m} \times \Delta^{n}$ such that $p^{*} A q^{*} \geq p A q^{*}$ for all $p \in \Delta^{m}$ and $p^{*} B q^{*} \geq p^{*} B q$ for all $q \in \Delta^{n}{ }^{6}$

\subsubsection{Loss Aversion}

Following Shalev (2000), we introduce loss aversion in two-player games $(A, B)$ by specifying nonnegative loss aversion coefficients $\lambda_{1}$ and $\lambda_{2}$, respectively measuring player 1's and player 2's degrees of loss aversion. A loss aversion bimatrix game is an object of the form $\left((A, B),\left(\lambda_{1}, \lambda_{2}\right)\right)$.

In addition to his loss aversion coefficient, player 1 [2] has a value $r_{1}\left[r_{2}\right]$ below which he considers the basic utility payoff entries of $A[B]$ as losses. These points, $r_{1}$ and $r_{2}$, are the players' respective reference points. The idea

\footnotetext{
${ }^{6}$ Note that we do not use the transposition notation for vectors and matrices if there is no confusion what is meant.
} 
of loss aversion is captured by transforming the players' basic utility payoffs as follows:

$$
\begin{aligned}
& a_{i j}^{\lambda_{1}, r_{1}}=a_{i j}-\lambda_{1} \max \left\{r_{1}-a_{i j}, 0\right\}, \\
& b_{i j}^{\lambda_{2}, r_{2}}=b_{i j}-\lambda_{2} \max \left\{r_{2}-b_{i j}, 0\right\} .
\end{aligned}
$$

Observe that this transformation preserves the ordering over deterministic payoffs. That is, a decision-maker prefers basic utility payoff $x$ to $y$ if and only if he prefers $x^{\lambda, r}$ to $y^{\lambda, r}$ for all $\lambda \geq 0$ and $r \in \mathbb{R}$.

For each equilibrium concept to be considered below, we require that it is a Nash equilibrium in the bimatrix game game $\left(A^{\lambda_{1}, r_{1}}, B^{\lambda_{2}, r_{2}}\right)$, where the reference points $r_{1}$ and $r_{2}$ are endogenous. The three equilibrium concepts differ in the way the reference points are determined.

\subsection{Fixed Point Loss Aversion Equilibrium}

Shalev (2000) introduces a loss aversion equilibrium where the players' reference points are found through a fixed point calculation. Define

$$
\underline{r}:=\min \left\{\min _{(i, j) \in I \times J} a_{i j}, \min _{(i, j) \in I \times J} b_{i j}\right\}
$$

and

$$
\bar{r}:=\max \left\{\max _{(i, j) \in I \times J} a_{i j}, \max _{(i, j) \in I \times J} b_{i j}\right\} .
$$

Thus, $\underline{r}$ and $\bar{r}$ are the lowest resp. the highest payoffs in $A$ or $B$. For a strategy profile $(p, q)$ and a reference point $r_{1} \in[\underline{r}, \bar{r}]$, player 1 has an expected payoff of $p A^{\lambda_{1}, r_{1}} q$. Observe that

$$
p A^{\lambda_{1}, \underline{r}} q=p A q \geq \min _{(i, j) \in I \times J} a_{i j} \geq \underline{r}
$$

and

$$
\bar{r} \geq \max _{(i, j) \in I \times J} a_{i j} \geq p A q \geq p A^{\lambda_{1}, \bar{r}} q .
$$

This and the fact that $p A^{\lambda_{1}, r_{1}} q$ is a continuous function of $r_{1}$ implies that there is an $r_{1}^{*} \in[\underline{r}, \bar{r}]$ such that $r_{1}^{*}=p A^{\lambda_{1}, r_{1}^{*}} q$. Furthermore, $r_{1}^{*}$ is unique because $r_{1}$ is strictly increasing on $[\underline{r}, \bar{r}]$, while $p A^{\lambda_{1}, r_{1}} q$ is nonincreasing on 
$[\underline{r}, \bar{r}]$. Similarly, there is a unique $r_{2}^{*} \in[\underline{r}, \bar{r}]$ such that $r_{2}^{*}=p B^{\lambda_{2}, r_{2}^{*}} q$. Clearly, these 'fixed point' reference points can be interpreted as the utilities players expect to realize given the strategy profile $(p, q)$. Hence, for each strategy profile $(p, q)$ there is a unique pair of reference points $\left(r_{1}, r_{2}\right)$ that is 'consistent' with this strategy profile.

Next, Shalev introduces a non-empty, compact- and convex-valued correspondence $\beta: \Delta^{m} \times \Delta^{n} \times[\underline{r}, \bar{r}]^{2} \rightarrow \Delta^{m} \times \Delta^{n} \times[\underline{r}, \bar{r}]^{2}$ where

$$
\begin{aligned}
& \beta\left(\hat{p}, \hat{q},\left(\hat{r}_{1}, \hat{r}_{2}\right)\right):=\left\{\left(p, q,\left(r_{1}, r_{2}\right)\right) \in \Delta^{m} \times \Delta^{n} \times[\underline{r}, \bar{r}]^{2}:\right. \\
& r_{1}=p A^{\lambda_{1}, \hat{r}_{1}} \hat{q} \geq p^{\prime} A^{\lambda_{1}, \hat{r}_{1}} \hat{q} \text { for all } p^{\prime} \in \Delta^{m}, \text { and } \\
&\left.r_{2}=\hat{p} B^{\lambda_{2}, \hat{r}_{2}} q \geq \hat{p} B^{\lambda_{2}, \hat{r}_{2}} q^{\prime} \text { for all } q^{\prime} \in \Delta^{n}\right\} .
\end{aligned}
$$

Since the (Nash) best reply-correspondence is upper semicontinuous and the players' payoff functions are continuous in their respective reference points, it follows that the correspondence $\beta$ is also upper semicontinuous. Hence, by the Kakutani fixed point theorem there exists a fixed point $\left(p^{*}, q^{*},\left(r_{1}^{*}, r_{2}^{*}\right)\right)$. Note that the strategy pair $\left(p^{*}, q^{*}\right)$ is a Nash equilibrium in the bimatrix game $\left(A^{\lambda_{1}, r_{1}^{*}}, B^{\lambda_{2}, r_{2}^{*}}\right)$. Since the reference points are determined through a fixed point calculation, we refer to this equilibrium concept as fixed point loss aversion equilibrium.

Definition 4.3.1. A fixed point loss aversion equilibrium $\left(p^{*}, q^{*}\right) \in \Delta^{m} \times$ $\Delta^{n}$ in a bimatrix game $\left((A, B),\left(\lambda_{1}, \lambda_{2}\right)\right)$, is a Nash equilibrium in the game $\left(A^{\lambda_{1}, r_{1}^{*}}, B^{\lambda_{2}, r_{2}^{*}}\right)$, where

$$
r_{1}^{*}=p^{*} A^{\lambda_{1}, r_{1}^{*}} q^{*} \text { and } r_{2}^{*}=p^{*} B^{\lambda_{2}, r_{2}^{*}} q^{*}
$$

One of the less attractive features of this equilibrium concept is that reference points are not unique: two loss aversion equilibria generally do not yield the same expected payoffs to the players. Furthermore, a player's reference point depends heavily on his own beliefs about the opponent's strategy. In what follows, we discuss two alternative equilibrium concepts which - to a greater or lesser extent - respond to these issues.

\subsection{Maximin Loss Aversion Equilibrium}

In maximin loss aversion equilibrium, each player chooses his reference point in such a way that his maximin payoff w.r.t. the strategies he believes 
his opponent plays with positive probability, is exactly equal to that reference point. The Nash equilibria in the game that results from using these consistent reference points are maximin loss aversion equilibria.

Maximin loss aversion equilibria are similar to fixed point loss aversion equilibria, in the sense that players base their reference points on the supports of their opponents' strategies. In a fixed point loss aversion equilibrium the reference points depend on the exact probabilities used in these strategies. In a maximin loss aversion equilibrium, the reference points depend only on the supports of the strategies of the opponents. Each player considers the pure strategies of the opponents that can be realized with positive probability, and his reference point is the pure maximin value over those strategies.

\subsubsection{Definition of Maximin Loss Aversion Equilibrium}

Formally, for a strategy combination $(p, q) \in \Delta^{m} \times \Delta^{n}$, the players' reference points are defined as follows. ${ }^{7}$

$$
r_{1}^{*}:=\max _{i \in I} \min _{j \in \operatorname{Car}(q)} a_{i j}, \text { and } r_{2}^{*}:=\max _{j \in J} \min _{i \in \operatorname{Car}(p)} b_{i j}
$$

Observe that this implies

$$
r_{1}^{*}:=\max _{i \in I} \min _{j \in \operatorname{Car}(q)} a_{i j}^{\lambda_{1}, r_{1}^{*}} \text { and } r_{2}^{*}:=\max _{j \in J} \min _{i \in \operatorname{Car}(p)} b_{i j}^{\lambda_{2}, r_{2}^{*}} .
$$

In other words, reference points do not change after the basic payoffs are transformed according to loss aversion with these reference points.

Reference points are unique for each support played by the opponent. Since a player's reference point only depends on the support of the strategy played by his opponent, rather than the strategy itself, reference points are more robust against wrong beliefs a player may have about his opponent.

Definition 4.4.1. A maximin loss aversion equilibrium in a loss aversion bimatrix game $\left((A, B),\left(\lambda_{1}, \lambda_{2}\right)\right)$ is a Nash equilibrium $\left(p^{*}, q^{*}\right) \in \Delta^{m} \times \Delta^{n}$ in the bimatrix game $\left(A^{\lambda_{1}, r_{1}^{*}}, B^{\lambda_{2}, r_{2}^{*}}\right)$, where $r_{1}^{*}$ and $r_{2}^{*}$ are the reference points for $\left(p^{*}, q^{*}\right)$ defined by (4.1).

\footnotetext{
${ }^{7}$ For simplicity of notation we do not use different symbols for the various reference point concepts.
} 
This concept of maximin loss aversion equilibrium does not solve the problem of multiple reference points. Because reference points no longer depend continuously on the strategies played by the opponent, maximin loss aversion equilibria may fail to exist.

\subsubsection{Existence of Maximin Loss Aversion Equilibrium}

\section{An Example Showing Non-Existence}

Consider the following $3 \times 3$ bimatrix game:

$$
A=\left[\begin{array}{ccc}
8 & 1 & 0 \\
1 & 8 & 0 \\
4 & 4 & -1
\end{array}\right] \quad B=\left[\begin{array}{lll}
1 & 2 & 1 \\
2 & 1 & 1 \\
0 & 0 & 1
\end{array}\right]
$$

Let player 2 be loss neutral, i.e. $\lambda_{2}=0$, and assume $\lambda_{1}=1$. Because of player 2's loss neutrality, $r_{2}$ has no influence on the equilibrium. That is, $B=$ $B^{\lambda_{2}, r_{2}}$ for all values of $r_{2}$. Observe that player 1's best reply against player 2 playing $e^{1}$ is $e^{1}$, and player 2 's best reply against this is $e^{2}$. Hence, $e^{1}$ can never be an equilibrium strategy for player 2 . Similarly, we can exclude $e^{2}$ as one of player 2's equilibrium strategies. This implies that player 1's equilibrium reference point is never equal to 8 . This leaves two possibilities: $r_{1}=0$ or $r_{1}=4$.

- $r_{1}=0$ : In this case, we have

$$
A^{\lambda_{1}, 0}=\left[\begin{array}{ccc}
8 & 1 & 0 \\
1 & 8 & 0 \\
4 & 4 & -2
\end{array}\right]
$$

The unique Nash equilibrium in $\left(A^{\lambda_{1}, 0}, B\right)$ is $((.5, .5,0),(.5, .5,0))$, implying $r_{1}=4$.

- $r_{1}=4:$ In this case, we have

$$
A^{\lambda_{1}, 4}=\left[\begin{array}{ccc}
8 & -2 & -4 \\
-2 & 8 & -4 \\
4 & 4 & -6
\end{array}\right]
$$

The unique Nash equilibrium in $\left(A^{\lambda_{1}, 4}, B\right)$ is $\left(\left(\frac{1}{3}, \frac{1}{3}, \frac{1}{3}\right),\left(\frac{1}{3}, \frac{1}{3}, \frac{1}{3}\right)\right)$, implying $r_{1}=0$. 
Each of player 1's possible reference points implies a loss aversion game in which the support of player 2's equilibrium strategy is such that another reference point should be chosen. Hence, there is no maximin loss aversion equilibrium.

\section{The Source of Non-Existence}

Define the players' respective maximin best reply correspondences $\beta_{1}: \Delta^{n} \rightarrow$ $\Delta^{m}$ and $\beta_{2}: \Delta^{m} \rightarrow \Delta^{n}$ as

$$
\begin{gathered}
\beta_{1}(\hat{q}):=\left\{p \in \Delta^{m}: p A^{\lambda_{1}, r_{1}} \hat{q} \geq p^{\prime} A^{\lambda_{1}, r_{1}} \hat{q} \text { for all } p^{\prime} \in \Delta^{m}\right. \text { and } \\
\left.r_{1}=\max _{i \in I} \min _{j \in \operatorname{Car}(\hat{q})} a_{i j}\right\},
\end{gathered}
$$

and

$$
\begin{gathered}
\beta_{2}(\hat{p}):=\left\{q \in \Delta^{n}: \hat{p} B^{\lambda_{2}, r_{2}} q \geq \hat{p} B^{\lambda_{2}, r_{2}} q^{\prime} \text { for all } q^{\prime} \in \Delta^{n}\right. \text { and } \\
\left.r_{2}=\max _{j \in J} \min _{i \in \operatorname{Car}(\hat{p})} b_{i j}\right\} .
\end{gathered}
$$

Define the overall maximin best-reply correspondence $\beta: \Delta^{m} \times \Delta^{n} \rightarrow \Delta^{m} \times$ $\Delta^{n}$ as $\beta:=\beta_{1} \times \beta_{2}$. For each $(\hat{p}, \hat{q})$ there is a single pair of payoff matrices $\left(A^{\lambda_{1}, r_{1}}, B^{\lambda_{2}, r_{2}}\right)$, and the set of best replies against $(\hat{p}, \hat{q})$ given these payoff matrices is non-empty, compact and convex. It follows that $\beta$ is non-empty, and compact- and convex-valued. Since a maximin loss aversion equilibrium need not exist, this implies that $\beta$ need not be upper semicontinuous. More specifically, there are sequences $\left(p^{k}, q^{k}\right)$ and $\left(\hat{p}^{k}, \hat{q}^{k}\right)$ in $\Delta^{m} \times \Delta^{n}$ converging to $\left(p^{*}, q^{*}\right)$ resp. $\left(\hat{p}^{*}, \hat{q}^{*}\right)$ and satisfying $\left(p^{k}, q^{k}\right) \in \beta\left(\hat{p}^{k}, \hat{q}^{k}\right)$ for all $k \in \mathbb{N}$ that do not satisfy $\left(p^{*}, q^{*}\right) \in \beta\left(\hat{p}^{*}, \hat{q}^{*}\right)$.

In our example, suppose player 2 has a sequence of strategies given by

$$
\hat{q}^{k}=\left[\begin{array}{c}
\frac{1}{3}+\frac{1}{6}\left(1-\frac{1}{k}\right) \\
\frac{1}{3}+\frac{1}{6}\left(1-\frac{1}{k}\right) \\
\frac{1}{3}-\frac{1}{3}\left(1-\frac{1}{k}\right)
\end{array}\right] .
$$

Note that this implies $r_{1}^{k}=0$ for all $k \in \mathbb{N}$. Then player 1 obtains $\frac{9}{2}-\frac{3}{2 k}$ from his first two strategies, and $4-\frac{2}{k}$ from his second. Thus, $\beta_{1}\left(\hat{q}^{k}\right)=\{(\alpha, 1-$ $\alpha, 0)$ with $\alpha \in[0,1]\}$ for all $k \in \mathbb{N}$. The sequence $\hat{q}^{k}$ converges to $\hat{q}^{*}=\left(\frac{1}{2}, \frac{1}{2}, 0\right)$. Then $r_{1}^{*}=4$ from which it follows that player 1 obtains a payoff of 3 for the first two strategies and 4 for the third one. Hence, $\beta_{1}\left(\hat{q}^{*}\right)=\{(0,0,1)\}$. Since $\beta_{1}$ does not satisfy upper semicontinuity, neither does $\beta$. 


\section{Existence in $m \times 2$ and $2 \times n$ Games}

Although maximin loss aversion equilibria do not exist in general, we do have existence in the case where one of the players has no more than two pure strategies.

Proposition 4.4.2. For all $\lambda_{1}, \lambda_{2} \geq 0$, and $m \times 2$ (or $2 \times n$ ) matrices $A$ and $B$, there exists a maximin loss aversion equilibrium in the loss aversion bimatrix game $\left((A, B),\left(\lambda_{1}, \lambda_{2}\right)\right)$.

Proof. Let $\lambda_{1}, \lambda_{2} \geq 0$ and let $A, B$ be $m \times 2$ matrices, and consider the game $\left((A, B),\left(\lambda_{1}, \lambda_{2}\right)\right)$. Define

$$
\tilde{r}:=\max _{i \in I} \min _{j \in J} a_{i j}
$$

and let $\left(p^{*}, q\right)$ be a Nash equilibrium in the game $\left(A^{\lambda_{1}, \tilde{r}}, B\right)$. We distinguish three (exhaustive) cases:

i. $q=e^{t}$ where $t \in J$. This implies there is an $s \in \mathrm{S}\left(p^{*}\right)$ such that $b_{s t}=$ $\max _{j \in J} b_{s j}$, since otherwise $e^{t}$ would not be a best reply of player 2 against $p^{*}$. Since loss aversion preserves the agent's preference ordering over pure alternatives, this implies $b_{s t}^{\lambda_{2}, b_{s t}}=\max _{j \in J} b_{s j}^{\lambda_{2}, b_{s t}}$. From the fact that $s \in \mathrm{S}\left(p^{*}\right)$, it follows that $a_{s t}^{\lambda_{1}, \tilde{r}}=\max _{i \in I} a_{i t}^{\lambda_{1}, \tilde{r}}$, and thus also $a_{s t}^{\lambda_{1}, a_{s t}}=\max _{i \in I} a_{i t}^{\lambda_{1}, a_{s t}}$. Hence, $\left(e^{s}, e^{t}\right)$ is a pure loss aversion equilibrium in $\left((A, B),\left(\lambda_{1}, \lambda_{2}\right)\right)$, and the associated reference point pair is $\left(a_{s t}, b_{s t}\right)$.

ii. $\left(p^{*}, q\right)=\left(e^{s},(\beta, 1-\beta)\right)$ where $s \in I$ and $\beta \in(0,1)$. This implies

$$
\beta a_{s 1}^{\lambda_{1}, r_{1}}+(1-\beta) a_{s 2}^{\lambda_{1}, r_{1}} \geq \beta a_{i 1}^{\lambda_{1}, r_{1}}+(1-\beta) a_{i 2}^{\lambda_{1}, r_{1}}
$$

for all $i \in I$ with $r_{1}=\tilde{r}$. Furthermore, it implies $b_{s 1}=b_{s 2}=: b$ from which it follows that $b_{s 1}^{\lambda_{2}, r_{2}}=b_{s 2}^{\lambda_{2}, r_{2}}$ with $r_{2}=b$. Hence, $\left(e^{s},(\beta, 1-\beta)\right)$ is a maximin loss aversion equilibrium in $\left((A, B),\left(\lambda_{1}, \lambda_{2}\right)\right)$, and the associated reference point pair is $(\tilde{r}, b)$.

iii. $p^{*}$ and $q$ satisfy $\left|\mathrm{S}\left(p^{*}\right)\right| \geq 2$ and $|\mathrm{S}(q)|=2$. Then there exist pure strategies $s$ and $s^{\prime}$ in $\mathrm{S}\left(p^{*}\right)$ such that $\left[b_{s 1}>b_{s 2}\right.$ and $\left.b_{s^{\prime} 1}<b_{s^{\prime} 2}\right]$ or $\left[b_{s 1}=b_{s 2}\right.$ and $\left.b_{s^{\prime} 1}=b_{s^{\prime} 2}\right]$. In both cases, there exists an $\alpha \in(0,1)$ such that both player 2's pure strategies are best replies against player 1 's strategy $\left(\alpha e^{s}+(1-\alpha) e^{s^{\prime}}\right)$, with payoffs given by $B^{\lambda_{2}, r_{2}}$ where $r_{2}=$ 
$\max _{j \in J} \min _{i \in\left\{s, s^{\prime}\right\}} b_{i j}$. But then $((\alpha, 1-\alpha), q)$ is a maximin loss aversion equilibrium in the game $\left((A, B),\left(\lambda_{1}, \lambda_{2}\right)\right)$. The associated reference points are

$$
r_{1}:=\max _{i \in\left\{s, s^{\prime}\right\}} \min _{j \in J} a_{i j} \text {, and } r_{2}:=\max _{j \in J} \min _{i \in\left\{s, s^{\prime}\right\}} b_{i j}
$$

respectively.

This completes the proof of the $m \times 2$ case. The $2 \times n$ case is analogous.

The above proposition includes $2 \times 2$ bimatrix games. It can be shown that the maximin best-reply correspondence for $2 \times 2$ bimatrix games satisfies upper semicontinuity, implying that existence in such cases also follows from Kakutani's fixed point theorem. Since for each $q \in \Delta^{2}$ there exists a $p \in \beta_{1}(q)$ with $|\mathrm{S}(p)|=2$, it seems one could obtain an easier proof for Proposition 4.4 .2 by restricting the best-reply correspondence $\beta_{1}$ to strategies with carriers containing at most two pure strategies. However, while such a restricted best-reply correspondence would not violate non-emptiness, it would definitely not be convex-valued. Hence, existence in the more general case of $m \times 2$ and $2 \times n$ games can not be obtained directly by means of Kakutani's fixed point theorem.

Note that the above proof provides a method to calculate maximin loss aversion equilibria. One can modify player 1's payoff matrix under the assumption that player 2 plays both his strategies with positive probability, and calculate the Nash equilibria in the resulting game. These Nash equilibria can then be transformed into maximin loss aversion equilibria.

\subsection{Safety Level Loss Aversion Equilibria}

In a safety level loss aversion equilibrium, a player's reference point is the value of the matrix game which is obtained by adapting his basic payoff matrix to account for loss aversion. If this basic payoff matrix is $C$ and the loss aversion coefficient is $\lambda$, then this reference point is equal to the number $r$ if $r$ is the value of the matrix game $C^{\lambda, r}$. Details are spelled out below.

This reference point does not depend on a player's belief about the strategy of the opponent in equilibrium. Instead, a player computes what he can obtain for sure and considers payoffs below this number as losses. Moreover, these reference points are unique, and safety level loss aversion equilibria always exist. 


\subsubsection{Definition and Existence of Safety Level Loss Aversion Equilibria}

The safety level is a concept that dates back to von Neumann's (1928) analysis of zero-sum games. Formally, for a bimatrix game $(A, B)$, the players' safety levels are defined by

$$
v_{1}(A):=\max _{p \in \Delta^{m}} \min _{q \in \Delta^{n}} p A q, \quad \text { and } \quad v_{2}(B):=\max _{q \in \Delta^{n}} \min _{p \in \Delta^{m}} p B q .
$$

Since a player can guarantee his safety level, it would make an intuitively appealing reference point in a loss aversion bimatrix game $\left((A, B),\left(\lambda_{1}, \lambda_{2}\right)\right)$. However, in the payoff matrices adapted by loss aversion the safety levels may change. Therefore, we look for reference points which in the transformed matrices are equal to the safety levels. That is, we wish to find $r_{1}^{*}$ and $r_{2}^{*}$ such that $r_{1}^{*}=v_{1}\left(A^{\lambda_{1}, r_{1}^{*}}\right)$ and $r_{2}^{*}=v_{2}\left(B^{\lambda_{2}, r_{2}^{*}}\right)$. Such reference points are called loss aversion safety levels.

To prove that there is a unique $r_{1}^{*}$ such that $r_{1}^{*}=v_{1}\left(A^{\lambda_{1}, r_{1}^{*}}\right)$, it is sufficient to show that $v_{1}\left(A^{\lambda_{1}, r}\right)$ is a continuous, nonincreasing function of $r$ on the interval $[\underline{r}, \bar{r}]$, and that

$$
v_{1}\left(A^{\lambda_{1}, \underline{r}}\right) \geq \underline{r}, \quad \text { and } \quad v_{1}\left(A^{\lambda_{1}, \bar{r}}\right) \leq \bar{r} .
$$

Clearly, $v_{1}\left(A^{\lambda_{1}, r}\right)$ is continuous in $r$. To show that it is nonincreasing in $r$ on the interval $[\underline{r}, \bar{r}]$, let $r_{1}$ and $s_{1}$ be reference points in $[\underline{r}, \bar{r}]$ with $r_{1}>s_{1}$, and let

$$
\begin{aligned}
& p^{*} \in \underset{p \in \Delta^{m}}{\arg \max _{q \in \Delta^{n}}} p A^{\lambda_{1}, r_{1}} q, \text { and } \\
& q^{*} \in \underset{q \in \Delta^{n}}{\arg \min ^{*} A^{\lambda_{1}, s_{1}} q .}
\end{aligned}
$$

Then

$$
\begin{aligned}
v_{1}\left(A^{\lambda_{1}, s_{1}}\right) & =\max _{p \in \Delta^{m}} \min _{q \in \Delta^{n}} p A^{\lambda_{1}, s_{1}} q \\
& \geq \min _{q \in \Delta^{n}} p^{*} A^{\lambda_{1}, s_{1}} q \\
& =p^{*} A^{\lambda_{1}, s_{1}} q^{*} .
\end{aligned}
$$

Note that $p A^{\lambda_{1}, r} q$ is nonincreasing in $r$ for any given strategy pair $(p, q)$. 
Therefore, $p^{*} A^{\lambda_{1}, s_{1}} q^{*} \geq p^{*} A^{\lambda_{1}, r_{1}} q^{*}$. Now observe that

$$
\begin{aligned}
p^{*} A^{\lambda_{1}, r_{1}} q^{*} & \geq \min _{q \in \Delta^{n}} p^{*} A^{\lambda_{1}, r_{1}} q \\
& =\max _{p \in \Delta^{m}} \min _{q \in \Delta^{n}} p A^{\lambda_{1}, r_{1}} q \\
& =v_{1}\left(A^{\lambda_{1}, r_{1}}\right) .
\end{aligned}
$$

It follows that $v_{1}\left(A^{\lambda_{1}, r}\right)$ is nonincreasing in $r$. Furthermore,

$$
v_{1}\left(A^{\lambda_{1}, \underline{r}}\right)=v_{1}(A) \geq \min _{(i, j) \in I \times J} a_{i j} \geq \underline{r}
$$

and since $v_{1}\left(A^{\lambda_{1}, r}\right)$ is nonincreasing in $r$, we also have

$$
v_{1}\left(A^{\lambda_{1}, \bar{r}}\right) \leq v_{1}\left(A^{\lambda_{1}, \underline{r}}\right)=v_{1}(A) \leq \max _{(i, j) \in I \times J} a_{i j} \leq \bar{r}
$$

Thus, there must be a unique $r_{1}^{*} \in[\underline{r}, \bar{r}]$ such that $r_{1}^{*}=v_{1}\left(A^{\lambda_{1}, r_{1}^{*}}\right)$. Similarly, there exists a unique $r_{2}^{*} \in[\underline{r}, \bar{r}]$ such that $r_{2}^{*}=v_{2}\left(B^{\lambda_{2}, r_{2}^{*}}\right)$.

The players transform their payoff matrices using their degrees of loss aversion and their loss aversion safety levels. The safety level loss aversion equilibria are the Nash equilibria in the transformed game, i.e. the bimatrix game $\left(A^{\lambda_{1}, r_{1}^{*}}, B^{\lambda_{2}, r_{2}^{*}}\right)$.

Definition 4.5.1. A safety level loss aversion equilibrium in a loss aversion bimatrix game $\left((A, B),\left(\lambda_{1}, \lambda_{2}\right)\right)$ is a Nash equilibrium $\left(p^{*}, q^{*}\right) \in \Delta^{m} \times \Delta^{n}$ in the bimatrix game $\left(A^{\lambda_{1}, r_{1}^{*}}, B^{\lambda_{2}, r_{2}^{*}}\right)$, where

$$
r_{1}^{*}=v_{1}\left(A^{\lambda_{1}, r_{1}^{*}}\right) \text { and } r_{2}^{*}=v_{2}\left(B^{\lambda_{2}, r_{2}^{*}}\right)
$$

\subsubsection{Strict Dominance when Players are Loss Averse}

In a safety level loss aversion equilibrium the determination of the equilibrium strategies is not related to the determination of the reference points. Hence, in contrast to the previous equilibrium concepts, reference points may also depend on strategies that are not played in equilibrium. Suppose for instance that player 2 has a strictly dominated column in his payoff matrix $B$, say $b_{i, n-1}>b_{i, n}$ for all $i=1, \ldots, m$. Then, whatever the reference level and the adapted payoff matrix are going to be, player 2 will not put any probability on column $n$ in an equilibrium. One could argue that player 1 , in determining his loss aversion safety level, should take into account that 
player 2 is not going to play column $n$. More generally, one could argue that, before actually computing loss aversion safety levels, first strictly dominated strategies should be iteratively eliminated. This raises the question which strategies are strictly dominated in the loss aversion context. The remainder of this section is devoted to studying this question.

We say that a pure strategy $i \in I$ is strictly dominated in $A$ if there is a strategy $p \in \Delta^{m}$ with $p_{i}=0$, such that $p A e^{j}>e^{i} A e^{j}$ for all $j \in J$. A pure strategy $i \in I$ is said to be strictly dominated in $\left(A, \lambda_{1}\right)$, if it is strictly dominated in $A^{\lambda_{1}, \rho}$ for all $\rho \in[\underline{r}, \bar{r}]$. Then, to eliminate a pure strategy $i \in I$ from the game, it is no longer sufficient that it is strictly dominated in $A$. To see this, consider the following example:

$$
A=\left[\begin{array}{ll}
5 & 0 \\
0 & 5 \\
2 & 2
\end{array}\right] .
$$

Observe that player 1's pure strategy $e^{3}$ is strictly dominated by the mixed strategy $(0.5,0.5,0)$. Now let $\lambda_{1}=1$ and $r_{1}=2$. Then the transformed payoff matrix is

$$
A^{1,2}=\left[\begin{array}{cc}
5 & -2 \\
-2 & 5 \\
2 & 2
\end{array}\right] .
$$

Clearly, strategy $e^{3}$ is no longer strictly dominated in $A^{1,2}$, even though it was in $A$.

Since $A=A^{\lambda_{1}, \underline{r}}, e^{i}$ being strictly dominated in $A$ is still a necessary condition for $e^{i}$ to be strictly dominated in $\left(A, \lambda_{1}\right)$. The following proposition gives a necessary and sufficient condition.

Proposition 4.5.2. In the game $\left((A, B),\left(\lambda_{1}, \lambda_{2}\right)\right)$ where $\lambda_{1}>0$, a strategy $p \in$ $\Delta^{m}$ strictly dominates the pure strategy $i \in I$ in $\left(A, \lambda_{1}\right)$ if and only if $p$ strictly dominates $i$ in $A^{\lambda_{1}, \rho}$ for all

$$
\rho \in\left[\min _{j \in J} a_{i j}, \max _{\left(i^{\prime}, j\right) \in \operatorname{Car}(p) \times J} a_{i^{\prime} j}\right] .
$$

Proof. Let a pure strategy $i$ be strictly dominated by a strategy $p$ in the payoff matrix $A$. Then define $\underline{\rho}:=\min _{j \in J} a_{i j}$ and $\bar{\rho}:=\max _{\left(i^{\prime}, j\right) \in \operatorname{Car}(p) \times J} a_{i^{\prime} j}$.

$\Leftarrow$ : Let the pure strategy $i$ be strictly dominated in $\left(A, \lambda_{1}\right)$. Then it is strictly dominated in $A^{\lambda_{1}, \rho}$ for all $\rho \in[\underline{r}, \bar{r}]$. Since $[\underline{\rho}, \bar{\rho}] \subseteq[\underline{r}, \bar{r}]$, strategy $i$ is strictly dominated in $A^{\lambda_{1}, \rho}$ for all $\rho \in[\underline{\rho}, \bar{\rho}]$. 
$\Rightarrow$ : Let the pure strategy $i$ be strictly dominated by $p$ in $A^{\lambda_{1}, \rho}$ for all $\rho \in[\rho, \bar{\rho}]$. Then $p$ strictly dominates $i$ in $A^{\lambda_{1}, \underline{\rho}}$. That is,

$$
p A^{\lambda_{1}, \underline{\rho}} e^{j}>e^{i} A^{\lambda_{1}, \underline{\rho}} e^{j}
$$

for all $j \in J$. Let $\rho \in[\underline{r}, \underline{\rho}]$. Then $e^{i} A^{\lambda_{1}, \rho} e^{j}=e^{i} A^{\lambda_{1}, \rho} e^{j}$. Furthermore, $\underline{\rho} \geq \rho$ implies $p A^{\lambda_{1}, \rho} e^{j} \leq p A^{\overline{\lambda_{1}, \rho}} e^{j}$. Hence, $p A^{\lambda_{1}, \rho} e^{j}>e^{i} A^{\lambda_{1}, \rho} e^{j}$ as well. So $p$ strictly dominates $i$ in $A^{\lambda_{1}, \rho}$ for all $\rho \in[\underline{r}, \underline{\rho}]$.

Similarly, $p$ strictly dominating $i$ in $A^{\lambda_{1}, \rho}$ for all $\rho \in[\rho, \bar{\rho}]$ implies that $p$ strictly dominates $i$ in $A^{\lambda_{1}, \bar{\rho}}$. That is, $p A^{\lambda_{1}, \bar{\rho}} e^{j}>e^{i} A^{\bar{\lambda}_{1}, \bar{\rho}} e^{j}$ for all $j \in J$. Observe that for all $\rho \in[\bar{\rho}, \bar{r}]$, we have that

$$
p A^{\lambda_{1}, \rho} e^{j}=\left(1+\lambda_{1}\right) p A e^{j}-\lambda_{1} \rho .
$$

Note that for all $j \in J$ there is an $i^{\prime} \in \mathrm{S}(p)$ such that $e^{i} A^{\lambda_{1}, \rho} e^{j} \leq e^{i^{\prime}} A^{\lambda_{1}, \rho} e^{j}$. Hence, for all $j \in J$ we have that $e^{i} A^{\lambda_{1}, \rho} e^{j} \leq \rho$ for all $\rho \in[\bar{\rho}, \bar{r}]$, implying

$$
e^{i} A^{\lambda_{1}, \rho} e^{j}=\left(1+\lambda_{1}\right) e^{i} A e^{j}-\lambda_{1} \rho
$$

for all $j \in J$ and $\rho \in[\bar{\rho}, \bar{r}]$. Hence, $\left(1+\lambda_{1}\right) p A e^{j}-\lambda_{1} \bar{\rho}>\left(1+\lambda_{1}\right) e^{i} A e^{j}-\lambda_{1} \bar{\rho}$ for all $j \in J$, and the inequality is preserved if we replace $\bar{\rho}$ by any $\rho \in[\bar{\rho}, \bar{r}]$. But then $p A^{\lambda_{1}, \rho} e^{j}>e^{i} A^{\lambda_{1}, \rho} e^{j}$ for all $j \in J$ and $\rho \in[\bar{\rho}, \bar{r}]$. That is, $p$ strictly dominates $i$ in $A^{\lambda_{1}, \rho}$ for all $\rho \in[\bar{\rho}, \bar{r}]$.

Thus, $p$ strictly dominates $i$ in $A^{\lambda_{1}, \rho}$ for all $\rho \in[\underline{r}, \bar{r}]$, which means that $p$ strictly dominates $i$ in $\left(A, \lambda_{1}\right)$.

Remark 4.5.3. It could happen that none of the $l$ strategies $p^{1}, \ldots, p^{l} \in \Delta^{m}$ dominate a pure strategy $i$ in $\left(A, \lambda_{1}\right)$, while taken together they do. Suppose none of the strategies $p^{1}, \ldots, p^{l}$ dominate $i$ in $\left(A, \lambda_{1}\right)$. Then for each $p^{k}$ there is an interval $R^{k} \subseteq[\underline{r}, \bar{r}]$ such that $p^{k}$ does not strictly dominate $i$ in $A^{\lambda_{1}, \rho}$ for any $\rho \in R^{k}$. Then, as long as $\bigcap_{k=1}^{l} R^{k}=\varnothing$, there is always a strategy $p^{k}$ strictly better than pure strategy $i$.

Thus, when considering safety level loss aversion equilibria in a loss aversion bimatrix game $\left((A, B),\left(\lambda_{1}, \lambda_{2}\right)\right)$, we could assume that the payoff matrices $A$ and $B$ are the result of iterated elimination of strategies that are either strictly dominated in $\left(A, \lambda_{1}\right)$ and $\left(B, \lambda_{2}\right)$ respectively, or strictly dominated in the weaker sense, explained in the above remark. 


\subsection{Comparative Statics}

In this section we consider the effect of loss aversion on the equilibrium payoff of a player. Specifically, suppose that both players are loss neutral but player 2 believes that player 1 is loss averse. This makes player 2 change his equilibrium strategy and we investigate when this is beneficial for player 1 .

\subsubsection{Preliminaries}

For reasons of tractability we only consider $2 \times 2$ bimatrix games. ${ }^{8}$ The basic utilities are represented by the following matrices:

$$
A:=\left[\begin{array}{ll}
a_{11} & a_{12} \\
a_{21} & a_{22}
\end{array}\right] \text { and } B:=\left[\begin{array}{ll}
b_{11} & b_{12} \\
b_{21} & b_{22}
\end{array}\right] .
$$

Since pure Nash equilibria are equivalent to the pure versions of all three types of loss aversion equilibrium considered above, we can restrict ourselves to mixed equilibria. Following Berden and Peters (2006), we exclude the case where player 1 has a weakly dominant strategy, implying $a_{11} \neq a_{21}$ and $a_{12} \neq a_{22}$. W.l.o.g. assume $a_{11}>a_{21}, a_{12}<a_{22}$, and $a_{11} \geq a_{22}$. This leaves three exhaustive cases:

i. $a_{21} \geq a_{22}$;

ii. $a_{22} \geq a_{21} \geq a_{12}$;

iii. $a_{12} \geq a_{21}$.

In addition, we assume $b_{11}<b_{12}$ and $b_{21}>b_{22}$. Hence, also player 2 has no weakly dominant strategy. These assumptions imply that the game has a unique, completely mixed Nash equilibrium $\left(p^{*}, q^{*}\right)$, with

$$
p^{*}=\left[\begin{array}{c}
\gamma \\
1-\gamma
\end{array}\right] \text { and } q^{*}=\left[\begin{array}{c}
\delta \\
1-\delta
\end{array}\right]
$$

where

$$
\gamma=\frac{b_{21}-b_{22}}{b_{12}-b_{11}+b_{21}-b_{22}} \text { and } \delta=\frac{a_{22}-a_{12}}{a_{11}-a_{12}-a_{21}+a_{22}} .
$$

Assume that player 2 does not have any information about his opponent's attitude towards losses, allowing him to form a wrong belief about it. That is,

\footnotetext{
${ }^{8} \mathrm{As}$ an additional advantage there is no existence problem for maximin equilibria.
} 
player 2 may falsely believe that player 1 is loss averse, which causes player 2 to play differently in order to make player 1 indifferent between his pure strategies in equilibrium. Specifically, player 2 plays a strategy $\tilde{q}=(\tilde{\delta}, 1-\tilde{\delta})$ where $\tilde{\delta} \in(0,1)$. Player 1 knows the utility function of his opponent, so he keeps playing his previous strategy $p^{*}$. Thus, if player 2 misperceives $\lambda_{1}$, the mixed loss aversion equilibrium becomes $\left(p^{*}, \tilde{q}\right) .^{9}$

The equilibrium under player 2's wrong perception of $\lambda_{1}$ can be explained in two ways. First, player 1 could be naive in the sense that he does not know that player 2 does not perceive his degree of loss aversion correctly. Thus, player 1 plays his equilibrium strategy and is surprised by player 2's action. A second explanation would be that player 1 - knowing that player 2 does not have any information about $\lambda_{1}$ - intentionally misrepresents his degree of loss aversion, but is myopic in the sense that he is not able to determine the strategy he has to play in order to optimally exploit player 2's action.

Clearly, starting from a situation where both players are loss averse but player 2 overestimates player 1's loss aversion is not really different from the present case.

\subsubsection{Result}

We say that player 2's wrong perception of $\lambda_{1}$ benefits player 1 if $p^{*} A \tilde{q} \geq$ $p^{*} A q^{*}$, and hurts him if $p^{*} A \tilde{q} \leq p^{*} A q^{*} .^{10}$ The following theorem presents the comparative statics result.

Theorem 4.6.1. In case $i$. player 1 benefits from player 2 misperceiving $\lambda_{1}$. In cases ii. and iii. player 1 benefits from player 2 misperceiving $\lambda_{1}$ if and only if

$$
\frac{b_{21}-b_{22}}{b_{12}-b_{11}+b_{21}-b_{22}} \geq \frac{a_{22}-a_{21}}{a_{11}-a_{12}+a_{22}-a_{21}} .
$$

The proof can be found in the appendix.

Note that the left-hand side in (4.2) is equal to $\gamma$, i.e., the probability that player 1 puts on the first row in equilibrium. If nonnegative, the right-hand side $\gamma^{\prime}=\frac{a_{22}-a_{21}}{a_{11}-a_{12}+a_{22}-a_{21}}$ in (4.2) is the probability that player 1 would have to

\footnotetext{
${ }^{9}$ In this $2 \times 2$ framework, players keep playing strategies with full supports for each type of loss aversion equilibrium.

${ }^{10}$ Note that player 2's wrong belief about his opponent's loss attitude neither hurts nor benefits him. That is, $p^{*} B q^{*}=p^{*} B \tilde{q}$.
} 
put on the first row in order to be indifferent between the actions of player 2 . In that case, playing the mixed strategy $p^{\prime}=\left(\gamma^{\prime}, 1-\gamma^{\prime}\right)$ would yield player 1 the Nash equilibrium payoff $p^{*} A q^{*}$. Thus, as long as player 2 plays his Nash equilibrium strategy $q^{*}$, then player 1 is indifferent between playing $p^{*}$ and $p^{\prime}$. However, if player 2 plays $\tilde{q}$, i.e. erroneously believes player 1 is loss averse, then player 1 obtains the Nash equilibrium payoff by playing $p^{\prime}$, but could receive more or less by playing $p^{*}$.

If condition (4.2) on the payoffs of the players is satisfied, we thus obtain that pretending to be more loss averse makes a player better off. The comparative statics of the fixed point loss aversion equilibrium were also investigated in Shalev (2000), with different results however. Shalev investigated how a player's reference point moves with his own degree of loss aversion. As this reference point equals a player's equilibrium payoff by definition, this yields associated comparative statics results. It is not clear what the proper interpretation of these results is since payoffs for players with different utility functions are compared. For our approach this difficulty does not arise since we compare payoffs of one and the same player 1: only player 2's belief about player 1 changes, but not player 1 himself.

\subsection{Summary}

We have argued in this chapter that in order to correctly incorporate the concept of loss aversion into noncooperative game theory, it is necessary to let the reference points of the players depend on the strategies the players play. Three different loss aversion equilibrium concepts were discussed, that satisfy this requirement. It was established that in $2 \times 2$ bimatrix games, a simple condition on the payoffs is sufficient for a player to benefit from his opponent overestimating his (the player's) degree of loss aversion.

\section{A Appendix: Proofs}

\section{A.1 The Proof of Theorem 4.6.1}

A player's reference point is in between the lowest and the highest payoff in his payoff matrix. In the $2 \times 2$ case, there are three intervals which could 
contain $r_{1}$ : the upper, the middle and the lower interval. Let $\pi \in \mathbb{R}^{4}$ with

$$
\pi= \begin{cases}\left(a_{12}, a_{22}, a_{21}, a_{11}\right) & \text { in case i. } \\ \left(a_{12}, a_{21}, a_{22}, a_{11}\right) & \text { in case ii. } \\ \left(a_{21}, a_{12}, a_{22}, a_{11}\right) & \text { in case iii. }\end{cases}
$$

The following lemma says that the reference point in a fixed point loss aversion equilibrium lies in the middle interval.

Lemma 4.A.1. If $\left(p^{*}, q^{*},\left(r_{1}, r_{2}\right)\right)$ is a fixed point loss aversion equilibrium in $\left((A, B),\left(\lambda_{1}, \lambda_{2}\right)\right)$, then $r_{1} \in\left[\pi_{2}, \pi_{3}\right]$.

Proof. For any basic utility payoff $x$, let $\hat{x}$ denote transformed payoff as a consequence of loss aversion. The expected payoff $r_{1}$ under the mixed fixed point loss aversion equilibrium is a convex combination of $\hat{a}_{21}$ and $\hat{a}_{22}$. Therefore, $r_{1} \leq \max \left\{\hat{a}_{21}, \hat{a}_{22}\right\} \leq \max \left\{a_{21}, a_{22}\right\}$ and thus, $r_{1} \leq \pi_{3}$.

To see that the reference point can never be in the lower interval, we consider each case separately.

Case i.: Here, $a_{21} \geq a_{22}$ and $a_{22}>a_{12}$. Assume $r_{1} \in\left[a_{12}, a_{22}\right)$. Note that $a_{22}-a_{21} \leq 0$ and $\hat{a}_{12}-a_{22}<0$, and thus $\left(a_{22}-a_{21}\right)\left(\hat{a}_{12}-a_{22}\right) \geq 0$. By an elementary computation it follows that

$$
\frac{a_{11} a_{22}-a_{21} \hat{a}_{12}}{a_{11}-\hat{a}_{12}-a_{21}+a_{22}} \geq a_{22} \text {. }
$$

In other words, player 1's expected payoff under loss aversion is larger or equal than $a_{22}$, contradicting $r_{1}<a_{22}$. Thus, $r_{1} \geq \pi_{2}$.

Case ii.: Here, $a_{11}>a_{21}$ and $a_{22} \geq a_{21}$. Assume $r_{1} \in\left[a_{12}, a_{21}\right)$. Now ( $a_{11}-$ $\left.a_{21}\right)\left(a_{22}-a_{21}\right) \geq 0$, which implies

$$
\frac{a_{11} a_{22}-a_{21} \hat{a}_{12}}{a_{11}-\hat{a}_{12}-a_{21}+a_{22}} \geq a_{21}
$$

contradicting $r_{1}<a_{21}$. Hence, $r_{1} \geq \pi_{2}$.

Case iii.: Repeating the argument from case ii., with $a_{21}$ replaced by $a_{12}$, and $\hat{a}_{12}$ by $\hat{a}_{21}$, yields the desired contradiction for case iii.

It follows from the above that $r_{1} \in\left[\pi_{2}, \pi_{3}\right]$.

For the other loss aversion equilibrium concepts we have similar results. 
Lemma 4.A.2. If $\left(p^{*}, q^{*},\left(r_{1}, r_{2}\right)\right)$ is a maximin loss aversion equilibrium in $\left((A, B),\left(\lambda_{1}, \lambda_{2}\right)\right)$, then $r_{1}=\pi_{2}$.

Proof. Since the equilibrium is completely mixed we have in Case i.: $\max _{i \in I} \min _{j \in J} a_{i j}=\max \left\{a_{12}, a_{22}\right\}=a_{22}$; and in Cases ii. and iii.: $\max _{i \in I} \min _{j \in J} a_{i j}=\max \left\{a_{12}, a_{21}\right\}=a_{21}$. Thus, $r_{1}=\pi_{2}$.

Lemma 4.A.3. If $\left(p^{*}, q^{*},\left(r_{1}, r_{2}\right)\right)$ is a safety level loss aversion equilibrium in $\left((A, B),\left(\lambda_{1}, \lambda_{2}\right)\right)$, then $r_{1} \in\left[\pi_{2}, \pi_{3}\right]$.

Proof. Recall that for any $2 \times 2$ matrix $A$, we have

$$
v_{1}(A)=\max _{p \in \Delta^{2}} \min _{q \in \Delta^{2}} p A q \geq \max _{i \in I} \min _{q \in \Delta^{2}} e^{i} A q=\max _{i \in I} \min _{j \in J} e^{i} A e^{j} .
$$

Assume $r_{1}<\pi_{2}$. Then

$$
\pi_{2}=\max _{i \in I} \min _{j \in J} e^{i} A^{\lambda_{1}, r_{1}} e^{j}
$$

In safety level loss aversion equilibrium we have $r_{1}=v_{1}\left(A^{\lambda_{1}, r_{1}}\right)$. Then

$$
v_{1}\left(A^{\lambda_{1}, r_{1}}\right)<\pi_{2}=\max _{i \in I} \min _{j \in J} e^{i} A^{\lambda_{1}, r_{1}} e^{j},
$$

which contradicts (4.3). Hence, $r_{1} \geq \pi_{2}$.

The safety level, $v_{1}\left(A^{\lambda_{1}, r_{1}}\right)$, can be interpreted as player 1's Nash equilibrium payoff in the zero-sum game $\left(A^{\lambda_{1}, r_{1}},-A^{\lambda_{1}, r_{1}}\right)$. By a similar reasoning as above, we have that player 2's payoff, $-v_{1}\left(A^{\lambda_{1}, r_{1}}\right)$, is above $-\pi_{3}$, implying $v_{1}\left(A^{\lambda_{1}, r_{1}}\right) \leq \pi_{3}$. Hence, $r_{1} \in\left[\pi_{2}, \pi_{3}\right]$.

Recall that $\tilde{q}=(\tilde{\delta}, 1-\tilde{\delta})$. We now compute $\tilde{\delta}$ for the three different cases.

Case i.: Here, we have $a_{11}>a_{21} \geq a_{22}>a_{12}$, and by Lemmas 4.A.1-4.A.3, $r_{1} \in\left[a_{22}, a_{21}\right]$. Thus,

$$
\begin{aligned}
\tilde{\delta} & =\frac{a_{22}-a_{12}-\lambda_{1}\left(r_{1}-a_{22}\right)+\lambda_{1}\left(r_{1}-a_{12}\right)}{a_{11}-a_{12}-a_{21}+a_{22}+\lambda_{1}\left(r_{1}-a_{12}\right)-\lambda_{1}\left(r_{1}-a_{22}\right)} \\
& =\frac{\left(1+\lambda_{1}\right)\left(a_{22}-a_{12}\right)}{a_{11}-a_{21}+\left(1+\lambda_{1}\right)\left(a_{22}-a_{12}\right)} .
\end{aligned}
$$


Case ii.: Here $a_{11}>a_{22} \geq a_{21}>a_{12}$ with $a_{11}>a_{22}, a_{22}>a_{21}$, or both. By Lemmas 4.A.1-4.A.3, we have $r_{1} \in\left[a_{21}, a_{22}\right]$. Hence,

$$
\begin{aligned}
\tilde{\delta} & =\frac{a_{22}-a_{12}+\lambda_{1}\left(r_{1}-a_{12}\right)}{a_{11}-a_{12}+\lambda_{1}\left(r_{1}-a_{12}\right)-a_{21}+\lambda_{1}\left(r_{1}-a_{21}\right)+a_{22}} \\
& =\frac{a_{22}-a_{12}+\lambda_{1}\left(r_{1}-a_{12}\right)}{a_{11}-a_{12}-a_{21}+a_{22}+\lambda_{1}\left(2 r_{1}-a_{12}-a_{21}\right)} .
\end{aligned}
$$

Case iii.: Here $a_{11}>a_{22} \geq a_{12}>a_{21}$. By Lemmas 4.A.1-4.A.3, we have $r_{1} \in\left[a_{12}, a_{22}\right]$, which implies that $\tilde{\delta}$ has the same value as in case ii. That is,

$$
\tilde{\delta}=\frac{a_{22}-a_{12}+\lambda_{1}\left(r_{1}-a_{12}\right)}{a_{11}-a_{12}-a_{21}+a_{22}+\lambda_{1}\left(2 r_{1}-a_{12}-a_{21}\right)} .
$$

Having specified $\tilde{q}$, player 2's equilibrium strategy associated with a wrong belief about $\lambda_{1}$, for each case, we now investigate how it compares to $q^{*}$, player 2's equilibrium strategy associated with the correct belief about $\lambda_{1}$.

Lemma 4.A.4. Let $\left(p^{*},(\delta, 1-\delta)\right)$ be the Nash equilibrium in $(A, B)$, and let $\left(p^{*},(\tilde{\delta}, 1-\tilde{\delta})\right)$ be the Nash equilibrium in $\left(A^{\lambda_{1}, r_{1}}, B\right)$, where $r_{1}$ is the equilibrium reference point associated with either of the three loss aversion equilibrium types. Then

$$
\tilde{\delta} \geq \delta
$$

in all three cases.

Proof. Let $x:=a_{22}-a_{12}$ and $y:=a_{11}-a_{21}-a_{12}+a_{22}$. Note that $x$ and $y$ are strictly positive, and that $\delta=x / y$. Assume $\lambda_{1}>0$. Again we consider the different cases.

Case i.: Here we have

$$
\tilde{\delta}=\frac{\left(1+\lambda_{1}\right)\left(a_{22}-a_{12}\right)}{a_{11}-a_{21}+\left(1+\lambda_{1}\right)\left(a_{22}-a_{12}\right)}=\frac{\left(1+\lambda_{1}\right) x}{y+\lambda_{1} x} .
$$

Since $y>x$ this implies $\tilde{\delta}>\delta$.

Case ii. and iii.: Here we have

$$
\tilde{\delta}=\frac{a_{22}-a_{12}+\lambda_{1}\left(r_{1}-a_{12}\right)}{a_{11}-a_{12}-a_{21}+a_{22}+\lambda_{1}\left(2 r_{1}-a_{12}-a_{21}\right)}=\frac{x+\lambda_{1}\left(r_{1}-a_{12}\right)}{y+\lambda_{1}\left(2 r_{1}-a_{12}-a_{21}\right)} .
$$

Now $\tilde{\delta} \geq \delta$ follows by straightforward computation, using $r_{1} \in\left[a_{21}, a_{22}\right]$. 
Proof of Theorem 4.6.1: We have

$$
p^{*} A q^{*}=\left[\begin{array}{ll}
\gamma a_{11}+(1-\gamma) a_{21} & \gamma a_{12}+(1-\gamma) a_{22}
\end{array}\right]\left[\begin{array}{c}
\delta \\
1-\delta
\end{array}\right],
$$

and

$$
p^{*} A \tilde{q}=\left[\begin{array}{ll}
\gamma a_{11}+(1-\gamma) a_{21} & \gamma a_{12}+(1-\gamma) a_{22}
\end{array}\right]\left[\begin{array}{c}
\tilde{\delta} \\
1-\tilde{\delta}
\end{array}\right] .
$$

From Lemma 4.A.4, we have $\tilde{\delta} \geq \delta$. Then $p^{*} A \tilde{q} \geq p^{*} A q^{*}$ if and only if $\gamma a_{11}+$ $(1-\gamma) a_{21} \geq \gamma a_{12}+(1-\gamma) a_{22}$, which is equivalent to (4.2). Observe that in case $i$. this condition is trivially satisfied. This concludes the proof. 


\section{Chapter 5}

\section{Relative Concave Utility for Risk and Ambiguity}

\subsection{Introduction}

Bernoulli (1954) introduced expected utility and diminishing marginal utility to explain risk aversion. Since then, it has been common in the literature to equate risk aversion and diminishing marginal utility. Frequently used indexes of risk aversion, such as the absolute and relative Arrow-Pratt indexes, refer exclusively to marginal utility, and comparisons of risk attitudes are captured by concave utility transformations (Arrow 1971; Pratt 1964; Yaari 1969). Similarly, Kreps and Porteus (1978) explained preference for or against early resolution of uncertainty by letting marginal utility depend on the time of resolution in a recursive expected utility model. Klibanoff, Marinacci, and Mukherji (2005; KMM), Nau (2006), and Neilson (1993) used a similar recursive expected utility model to explain ambiguity attitudes such as exhibited by the Ellsberg paradox in terms of marginal utility.

This chapter presents a discrete version of marginal rates of substitution for analyzing utility, and applies it to the models of the afore-mentioned references. This is first related to a known tradeoff tool (Köbberling and Wakker 2003), and more widely applicable preference axiomatizations for all afore-mentioned models are obtained. Next it is used to obtain a general method for comparing the concavity of different utility functions. Although the method is elementary in a mathematical sense, it delivers many generalizations of the classical results in the literature that have not been known before. We, thus, extend Yaari's (1969) comparability of risk attittudes to 
the case of different subjective beliefs. We similarly extend KMM's smooth ambiguity comparisons to the case of different subjective beliefs and different risk attitudes. For KMM's model, we further avoid the use of theoretical constructs that are not directly observable - such as subjective probabilities - in the preference axioms. For the recursive expected utility models, we need not commit to violations of the reduction of compound lotteries, and we do not need known probabilities.

Thus, a first contribution this chapter makes, concerns the utility analysis in a number of popular models, which is expected to be uncontroversial to all readers. For readers who do not doubt the appropriateness of marginal utility for completely capturing attitudes towards risk and ambiguity, the technique presented in this chapter is a contribution to the analysis of such attitudes. For other readers, however, the interpretation of the results may be different when it comes to the analysis of risk and ambiguity attitudes. Discrete rates of substitution isolate the empirical meaning of utility very clearly. As illustrated in Figure 5.2(a), uncovering this empirical meaning, requires carefully inspecting many outcomes, with almost no regard of the events or the risks. It does not seem plausible that detailed inspections of outcomes could suffice to completely capture attitudes towards ambiguity or risk. The same basic objection was raised against expected utility under risk in the early 1980's (Schoemaker 1982), and was a reason for developing nonexpected utility. This classical argument against expected utility is sharpened and extended to the use of expected utility for ambiguity. Thus, the results of this chapter support models for ambiguity, that use nonexpected utility. Such models may use nonadditive probabilities (Gilboa 1987; Schmeidler 1989), or multiple priors (Gilboa and Schmeidler 1989) as well as robust control (Hansen and Sargent 2001; Maccheroni, Marinacci, and Rustichini 2006). The latter models put the processing of uncertain events central in their analyses of risk and ambiguity, and thus avoid the aforementioned intuitive objection.

This chapter continues as follows. Section 5.2 informally presents a discrete version of marginal rates of substitution. It shows how this version can be used to analyze risk and uncertainty, and prepares for some intuitive discussions. Section 5.3 presents basic definitions of decision under under uncertainty, and Section 5.4 adds technical definitions. Section 5.5 defines a tradeoff indifference that formalizes the discrete rates of substitution, and states the main results, to be applied in the following sections. Section 5.6 generalizes the results of Yaari (1969) on comparative risk aversion. Section 
5.7 axiomatizes a recursive model of Kreps and Porteus (1978) and of KMM, and derives some absolute concavity results. Relative results for the recursive model are in Section 5.8, and Section 5.9 compares the results of the chapter to related results in the literature. Section 5.10 contains a discussion, and Section 5.11 concludes.

\subsection{Risk Aversion through Marginal Rates of Sub- stitution}

In consumer theory, marginal rates of substitution (MRS) provide a useful tool for analyzing utility. For example, if at the starting point $(x, \gamma)$ in figure 5.1(a), an increase of $\beta-\gamma$ infinitesimal units of one commodity, say tea (T), exactly offsets an increase of one infinitesimal unit $y-x$ of Hats $(\mathrm{H})$, then the MRS of $\mathrm{H}$ for $\mathrm{T}$ is $\beta-\gamma$. The common empirical finding is that MRS's diminish, corresponding with concave utility. This implies that, at the higher starting point $(x, \beta)$ in Figure 5.1(b), the MRS of $\mathrm{H}$ for T, $\alpha-\beta$, exceeds $\beta-\gamma$. The more tea you have, the more extra tea you need to offset one unit of hats. Under cardinal utility models, the utility midpoint between $\alpha$ and $\gamma$, i.e. $\beta$, then is below the physical midpoint, i.e. $\beta \leq \frac{\alpha+\gamma}{2}$. Formally, the condition then implies that the second derivative of utility with respect to $\mathrm{T}$ is negative; that is, utility is concave in T. Figure 5.1(c) presents a comparative result at $v$ versus $x(v>x$ and $w-v=y-x)$. The utility midpoint at $v, \beta^{*}$, is lower than $\beta$, the utility midpoint at $x$. This implies that the utility of tea is more concave for $v$ hats than for $x$ hats. The lower the midpoint $\beta$ is, the more concave utility is. We can similarly compare MRS's and concavity of utility by comparing utility midpoints not for different levels of hats, but for different persons, or within one person for different circumstances (for different timepoints, and so on), as we will elaborate on later.

In decision theory, we usually observe discrete preferences rather than first order optimality conditions. Then, we use discrete versions of Figures 5.1(a) and 5.1(b) where $y-x, \beta-\gamma, \alpha-\beta$, and $w-v$ are not infinitesimal, but take real values. The quantities $\frac{\alpha-\beta}{y-x}$ and $\frac{\beta-\gamma}{y-x}$ of Figure 5.1(b) can now be taken as two discrete rates of substitution (RS). For cardinal utility models, utility of $\mathrm{T}$ is still concave for $\mathrm{H}$ fixed at $x$ if and only if in all configurations of Figure 5.1(b) - also discrete ones - we have $\beta \leq \frac{\alpha+\gamma}{2}$. This condition, called Diminishing Rate of Substitution (DRS) will be formalized later. Similarly, noninfinitesimal distances are now considered in Figure 5.1(c), and $\beta^{*} \leq \beta$ still characterizes relative concave utility. 


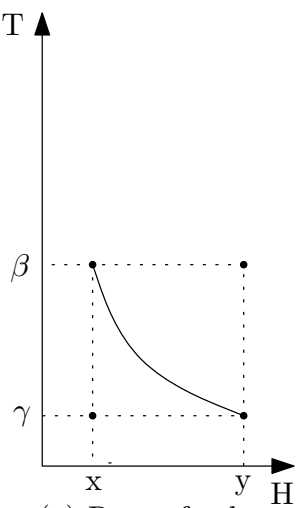

(a) Rate of substitution

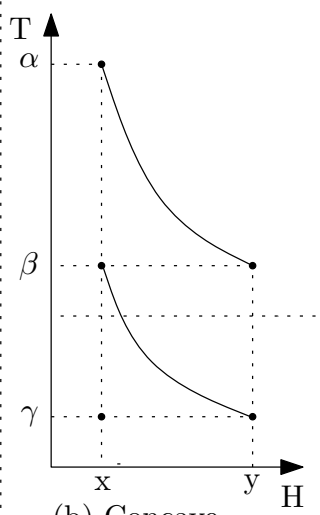

(b) Concave utility for $\mathrm{T}$

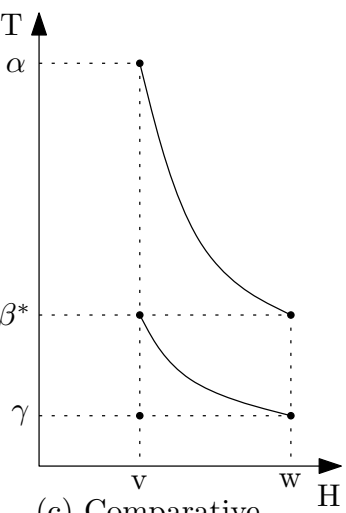

(c) Comparative $\mathrm{RS}: \beta^{*} \leq \beta$

Figure 5.1: Rates of Substitution

As pointed out by Arrow (1964) and Debreu (1959), techniques used to analyze preferences over commodity bundles can also be used to analyze preferences under uncertainty. Suppose a coin is flipped, with either heads (event $\mathrm{H}$ ) or tails (event $\mathrm{T}$ ) coming up. The point $(x, \gamma)$ now designates an event-contingent payment, yielding $x$ if $\mathrm{H}$, and $\gamma$ if T. Such event-contingent payments are called acts. Under expected utility, the act $(x, \gamma)$ is evaluated by $p U(x)+(1-p) U(\gamma)$, where $p$ denotes the probability of $\mathrm{H}$, and $U$ denotes utility. Concave utility now characterizes risk aversion, i.e. a preference for expectations over acts. Using the Arrow-Debreu analogy, we see that DRS, as illustrated in Figure 5.1(b), is equivalent to risk aversion. Further, one decision maker is more risk averse than another if and only if the corresponding utility midpoints are lower at the earlier time point. Hence, under expected utility, we can analyze risk attitude, using RS's - well known from consumer theory - rather than risk premiums.

Some readers might raise an intuitive objection against the preceding analysis of risk attitudes in terms of RS's. After all, RS's only concern tastes for outcomes, as they do in consumer theory, and they do not refer to risk in any direct manner. The only probability considered above was $p$, the probability of heads, and its role was minimal. In fact, the whole analysis works irrespectively of what $p$ is, and we need not know $p$. It is not plausible that risk attitude can be captured completely without any consideration for the role of probabilities. To endorse this intuitive objection, however, the ex- 
pected utility model has to be abandoned. Under expected utility, it follows as a logical truh that RS or, equivalently, marginal utility, captures risk attitude. The intuition that utility, reflecting the taste for outcomes, cannot capture risk attitudes was one of the reasons why generalizations of expected utility were introduced in the 1980's. It implies in particular that comparisons of marginal utility cannot capture comparisons of risk attitudes.

For readers who do not share the intuitive objection and who adhere to expected utiltiy, RS's simply provide a useful alternative to risk premiums for analyzing risk attitudes. There is an additional advantage of RS's over risk premiums for decision under uncertainty, where probabilities are not given beforehand but have to be subjective. Then - unlike with risk - the probabilities are not directly observable. Instead, they are theoretical constructs that have to be derived from preferences. Preference conditions that use subjective probabilities as inputs are not directly testable, much the same as preference conditions that use utilities as inputs are not directly testable. There is no easy way to calculate expected values or risk premiums if we do not know the subjective probabilities of a person. RS's, to the contrary, are as readily observable under uncertainty as they are under risk; and, as they are in consumer theory. They continue to serve as useful tools for analyzing risk attitude also when probabilities are subjective. Indeed, the whole analysis of Figure 5.1(c) remains valid and directly observable even if $p$ is subjective and unknown.

\subsection{Elementary Definitions}

This section gives the basic definitions for decision under uncertainty. $S$ denotes the state space, which can be finite or infinite. Exactly one state $s \in S$ is true, the other states are not true, and it is uncertain which state is the true one. An algebra is a collection of subsets of $S$ which contains $\varnothing$ and $S$, and which is closed under complement taking and finite unions and intersections. We assume that an algebra $\mathscr{A}$ is given on $S$, the elements of which are called events. All sources considered later will be subalgebras of $\mathscr{A}$.

The outcome set $\mathscr{C}$ is a nondegenerate subinterval of $\mathbb{R}$, with outcomes being monetary. Outcomes are denoted by Greek letters $\alpha, \beta$, etc. Preferences will be defined over a set $X$ of acts. Acts, denoted $x, y, \ldots$, are measurable mappings from states to outcomes; $x$ assigns outcome $x(s)$ to each state $s \in S$. Measurability means that the inverse of every subinterval of $\mathscr{C}$ 
is contained in $\mathscr{A}$. Readers not interested in measure theory may assume that $\mathscr{A}$ contains all subsets of $S$, in which case all functions from $S$ to $\mathscr{C}$ are measurable so that all measurability considerations are trivially satisfied and can be ignored.

Each outcome $\alpha$ is identified with the constant act $x$ with $x(s)=\alpha$ for all $s$. We assume that all finite-valued measurable mappings from $S$ to $\mathscr{C}$ (called simple acts) are contained in $X .\left(E_{1}: x_{1}, \ldots, E_{n}: x_{n}\right)$ denotes the simple act assigning $x_{j}$ to each $s$ in $E_{j}$; the $E_{j}$ 's partition $S$. Other than that, we allow $X$ to be almost any subset of the set of measurable mappings from $S$ to $\mathscr{C}$. One more restriction on $X$, to ensure that all expected utilities considered hereafter are well-defined and finite, will be added later.

A preference relation of a decision maker is a binary relation over $X$, denoted by $\succcurlyeq$. The notation $>, \sim, \preccurlyeq$, and $\prec$ is as usual. Expected utility (EU) holds if there exist a (finitely additive) probability measure $P$ on $\mathscr{A}$, and a strictly increasing utility function $U: \mathbb{R} \rightarrow \mathbb{R}$, such that: (a) $E U(x)=$ $\int_{S} U(x(s)) d P(s)$ (the expected utility of act $x$ ) is well-defined and finite for all $x \in X$; and (b) $x \succcurlyeq y$ if and only if $E U(x) \geq E U(y)$. EU implies that $\succcurlyeq$ is a weak order, i.e. it is complete (for all $x, y$, either $x \succcurlyeq y$ or $y \succcurlyeq y$ ) and transitive (for all $x, y, z$, we have that $x \succcurlyeq y$ and $y \succcurlyeq z$ together imply $x \succcurlyeq z$ ).

A certainty equivalent of an act $x$ is an outcome $\alpha$ such that $\alpha \sim x$. Under EU with continuous strictly increasing utility, $\alpha=U^{-1}(E U(x))$ is unique. For outcome $\alpha$, event $E$, and act $x, \alpha_{E} x$ denotes the act assigning outcome $\alpha$ to each state in event $E$ and the same outcome as $x$ to each state off $E$. Thus, given that $\beta$ can denote a constant act, $\alpha_{E} \beta$ denotes the two-outcome act $(E: \alpha$, not- $E: \beta)$.

\subsection{Technical Definitions}

This section defines technical conditions. It can be skipped by readers interested primarily in empirical and conceptual results. An event $E$ is null if $\alpha_{E} x \sim \beta_{E} x$ for all acts $x$ and outcomes $\alpha$ and $\beta$, and nonnull otherwise. Under EU, an event $E$ is null if and only if $P(E)=0$. We assume nondegeneracy throughout, implying that there exists a nonnull event $E$ for which the complement is also nonnull. Under EU, nondegeneracy is equivalent to $0<P(E)<1$. We also assume monotonicity, i.e. $x \succcurlyeq y$ if $x(s) \geq y(s)$ for each state $s$, with $x>y$ if $x(s)>y(s)$ for each state $s$. Monotonicity implies that $\alpha \succcurlyeq \beta$ if and only if $\alpha \geq \beta$ for all outcomes $\alpha, \beta$. EU implies monotonicity.

We avoid infinite-dimensional topological complications by restricting 
continuity to finite-dimensional subspaces: $\succcurlyeq$ is continuous if, for every partition $\left(E_{1}, \ldots, E_{n}\right)$ of $S$, the preference relation restricted to acts $\left(E_{1}\right.$ : $\left.x_{1}, \ldots, E_{n}: x_{n}\right)$ satisfies the usual Euclidean continuity. Under EU, continuity of preference can be seen to be equivalent to continuity of utility.

To avoid undefined or infinite EU values, and to express this requirement in directly observable preference conditions, we define truncations of acts, following (Wakker 1993). All truncation conditions defined hereafter are trivially satisfied if all acts $x \in X$ are bounded in the sense that there exists an outcome preferred to all $x(s)$ and one less preferred than all $x(s)$. This includes the case where $X$ contains only the simple acts. Readers only interested in bounded acts may skip the following definitions concerning truncations. For act $x$ and outcome $\mu, x \wedge \mu$, the above truncation of $x$ at $\mu$, assigns $x(s)$ to $s$ whenever $x(s) \preccurlyeq \mu$ and it assigns $\mu$ to $s$ whenever $x(s)>\mu$. For act $x$ and outcome $\eta, x \vee \eta$, the below truncation of $x$ at $\eta$, assigns $x(s)$ to $s$ whenever $x(s) \succcurlyeq \eta$ and it assigns $\eta$ to $s$ whenever $x(s)<\eta$. $X$ is truncation-closed if all (above and below) truncations of all of its acts are contained in $X$. Truncation continuity holds if, whenever $x>y$ for a simple act $y$, then $x \wedge \mu>y$ for some outcome $\mu$ and, whenever $x<y$ for a simple act $y$, then $x \vee \eta<y$ for some outcome $\eta$.

It is sometimes convenient if probability measures are countably additive. A probability measure is countably additive if $A_{j} \downarrow \varnothing\left(A_{j} \supset A_{j+1}\right.$ and the intersection of these sets is empty) implies that $P\left(A_{j}\right)$ converges to 0 . It is equivalent to the probability of a countable disjoint union being the sum of the individual probabilities whenever that countable union is contained in the algebra. The condition is most useful if the algebra $\mathscr{A}$ is a sigma-algebra (i.e. an algebra closed under countable unions). A preference condition necessary and sufficient for countable additivity is set-continuity:

If $\beta<\gamma, A_{j} \downarrow \varnothing$, and $x>\beta$ then, for some natural number $J$, $x>\gamma_{A_{j}} \beta$ for all $j>J$.

We summarize the assumptions made.

Structural Assumption 5.4.1 (Decision under Uncertainty). $S$ is a state space endowed with an algebra $\mathscr{A}$ of subsets called events, and $\mathscr{C}$, a subinterval of $\mathbb{R}$, is the outcome set. $X$, the set of acts, contains all simple measurable mappings from $S$ to $\mathscr{C}$, and possibly some other measurable mappings from $S$ to $\mathscr{C}$. $\succcurlyeq$ is a binary relation on $X$. Truncation closedness holds, and for every act there exists a certainty equivalent. Nondegeneracy holds. 
We will sometimes assume that objective probabilities of the events are available, in which case by common assumptions of decision under risk the act can be identified with the probability distribution generated over the outcomes. Then $\alpha_{p} \beta$ denotes the lottery yielding $\alpha$ with probability $p$ and $\beta$ with probability $1-p$.

Structural Assumption 5.4.2 (Decision under Risk). Structural Assumption 5.4.1 holds. An (objective) probability measure is given on $\mathscr{A}$. The preference value of an act depends on the lottery generated over the outcomes, and every simple lottery over outcomes is generated by some act.

\subsection{Formalizing Rates of Substitution}

\subsubsection{Rates of Substitution Defined Formally}

We formalize some claims of Section 5.2 about rates of substitution, and relate those rates to a tradeoff indifference tool of Köbberling and Wakker (2003). We next present some generalizations of existing results that will be used at a later point in the chapter. We write

$$
[\alpha ; \beta] \sim^{*}[\mu ; v] \text { or } \alpha \beta \sim^{*} \mu v
$$

if

$$
\begin{aligned}
& \alpha_{E} x \sim \beta_{E} y \text { and } \\
& \mu_{E} x \sim v_{E} y .
\end{aligned}
$$

for some nonnull event $E$ and simple acts $x$ and $y . \sim^{*}$ is called a tradeoff indifference. In Figure 5.1(b) we have $\alpha \beta \sim^{*} \beta \gamma$ (take $E=\mathrm{T}, \mu=\beta$, and $v=$ $\gamma)$. Here, each side of a tradeoff indifference concerns the numerator $(\alpha-\beta$ and $\beta-\gamma$ respectively) of the RS of money-contingent-on-not- $E$ for moneycontingent-on- $E\left(\frac{\alpha-\beta}{y-x}\right.$ and $\frac{\beta-\gamma}{y-x}$, respectively $)$ with the denominator of each $\mathrm{RS}$ being the same. Therefore, testing if the RS is increasing or decreasing is easy, simply by comparing $\alpha-\beta$ with $\beta-\gamma$.

Definition 5.5.1. Diminishing rate of substitution (DRS) holds if

$$
\alpha \beta \sim^{*} \beta \gamma \Rightarrow \beta \leq \frac{\alpha+\gamma}{2} .
$$


Because of symmetry, no ordering between $\alpha$ and $\gamma$ has to be imposed in Definition 5.5.1.

In the following definition, as throughout the rest of the chapter, superscripts indicate indexes and not powers. The definition considers two preference relations and formalizes Figure 5.1(c).

Definition 5.5.2. $\succcurlyeq^{1}$ exhibits a stronger DRS than $\succcurlyeq^{2}$ if

$$
\alpha \beta \sim^{* 2} \beta \gamma \text { and } \alpha \beta^{\prime} \sim^{* 1} \beta^{\prime} \gamma \Rightarrow \beta^{\prime} \leq \beta .
$$

A tradeoff relation was used in axiomatizations of several models of decision under uncertainty by Köbberling and Wakker (2003). We similarly use it to discretize MRS's so that we can analyze properties of utility and relate those to risk (and ambiguity) attitudes. From substitution, we get:

Lemma 5.5.3. Under $E U, \alpha \beta \sim^{*} \mu v$ if and only if $U(\alpha)-U(\beta)=U(\mu)-U(v)$.

\subsubsection{Measuring Utility using Rates of Substitution}

In an experiment, Wakker and Deneffe (1996) measured for a nonnull event $E$, a sequence of indifferences

$$
\alpha_{E}^{j} x \sim \alpha_{E}^{j-1} y, \text { where } j=1, \ldots, n ;
$$

see Figure 5.2(a). In our notation, this implies

$$
\alpha^{j+1} \alpha^{j} \sim^{*} \alpha^{1} \alpha^{0} \text { for all } j=1, \ldots, n-1 .
$$

By Lemma 5.5.3, the $\alpha^{j}$ 's are equally-spaced in utility units. Normalizing $U\left(\alpha^{0}\right)=0$ and $U\left(\alpha^{n}\right)=1$, we obtain $U\left(\alpha^{j}\right)=j / n$ for all $j$, and the graph of $U$ in Figure 5.2(b) results (depicted for $n=4$ ).

Thus, RS's underly Wakker and Deneffe's tradeoff method, which provides an easy tool for empirically measuring utility, even if we do not know the subjective probability of event $E$. We can measure $U$ on as a large an interval as we want by taking $\alpha^{0}$ as small as needed and $\alpha^{n}$ as large as needed. We can have the measurement as refined as we want by taking $x$ and $y$ outside of $E$ sufficiently close to each other so that the differences between the $\alpha$ 's become as small as desired. In this manner, we can get to know everything about $U$ that we want to.

One key aspect of the utility measurement technique just described is central to this chapter. It is that the procedure inspects outcomes $\alpha^{0}, \ldots, \alpha^{n}$ in great detail, but hardly pays attention to the events or probabilities, be they objective or subjective (Eq. (5.3) and Figure 5.2(b)). 


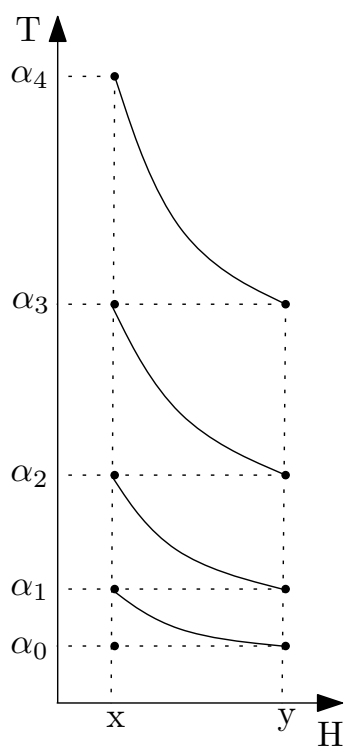

(a) Eq. (5.3).

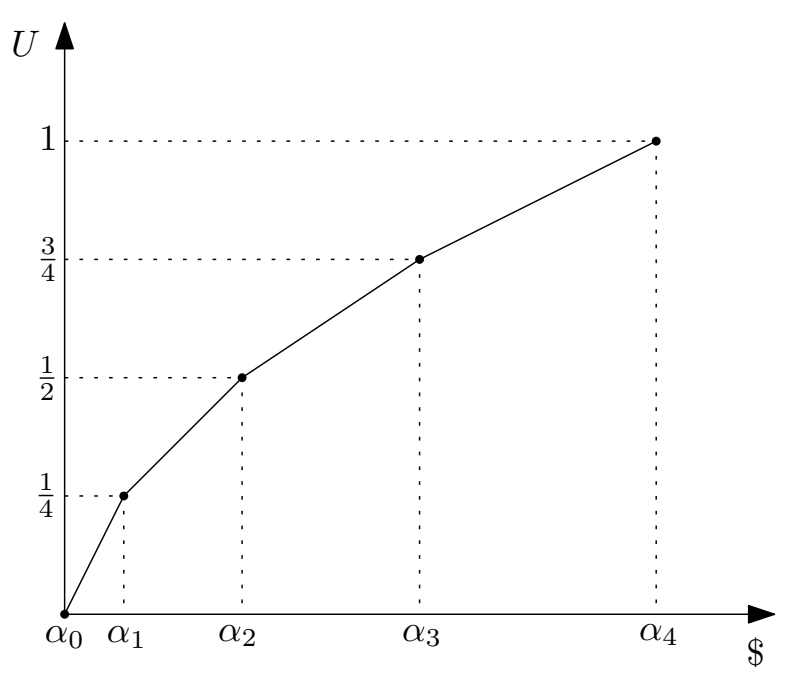

(b) Utility graph derived from preference midpoint observations.

Figure 5.2: Measuring utility requires a detailed analysis of outcomes, not of events.

Conclusion 5.5.4. Under EU, utility can be analyzed without inspecting the probabilities or ambiguous events.

\subsubsection{Preference Foundations}

We now turn to a preference foundation for EU using DRS. The following consistency condition for utility measurement is obviously necessary for EU to hold, given that utility is strictly increasing.

Definition 5.5.5. Tradeoff consistency holds if improving an outcome in any $\alpha \beta \sim^{*} \gamma \delta$ relationship breaks that relationship.

That is, if $\alpha \beta \sim^{*} \gamma \delta$ and $\alpha^{\prime} \beta \sim^{*} \gamma \delta$, then we must have $\alpha=\alpha^{\prime}$. Although the definition uses the derived concept of $\sim^{*}$, it is easily reformulated directly in terms of preferences so that it is directly testable and can be used in preference foundations. Tradeoff consistency provides a preference foundation for expected utility. The following result generalizes results of Wakker (2009) to nonsimple acts, and results of Wakker (1993) to the less restrictive tradeoff condition used here. 
Theorem 5.5.6. Under the Structural Assumption 5.4.1 (decision under uncertainty), the following two statements are equivalent.

(i) Expected utility holds with continuous strictly increasing utility.

(ii) $\succcurlyeq$ satisfies:

(a) weak ordering;

(b) monotonicity;

(c) continuity;

(d) truncation continuity;

(e) tradeoff consistency.

In Statement (i), the probabilities are unique and the utility function is unique up to level and unit. The probability in Statement (i) is countably additive if and only if set-continuity holds.

Utility need not be bounded in Theorem 5.5.6. Given that utility is determined up to level and unit, we will throughout equate equivalent utility functions and will write $U^{1}=U^{2}$ when $U^{1}=\tau+\sigma U^{2}$ for a real $\tau$ and a positive $\sigma$. The following corollary adapts Theorem 5.5.6 to decision under risk.

Corollary 5.5.7. Under the Structural assumption 5.4 .2 (decision under risk), the following two statements are equivalent:

(i) Expected utility holds with continuous strictly increasing utility and the (subjective) probabilities used in the EU model of Theorem 5.5.6 identical to the objective probabilites.

(ii) $\succcurlyeq$ satisfies:

(a) weak ordering;

(b) monotonicity;

(c) continuity;

(d) truncation continuity;

(e) tradeoff consistency.

The utility function in Statement (i) is unique up to level and unit. 
The characterization of expected utility by von Neumann and Morgenstern (1944) is very appealing. We presented the above alternative characterization because we need the $\sim^{*}$ relation for other purposes later, and this way we obtain a unified analysis. Relative to Fishburn's (1970, Theorem 10.1) version of the von Neumann-Morgenstern theorem, we have generalized the result to allow for unboundedness of utility.

\subsubsection{Risk Aversion Analyzed using DRS}

The expected value needed for the definition of risk aversion can only be defined if probabilities over the state space $S$ can be defined. This is the case for decision under risk, and decision under uncertainty if EU holds. Then a risk premium designates the difference between expected value and the certainty equivalent of a lottery.

Quasi-concavity of preferences in terms of mixing provides an alternative characterization of risk aversion for unknown probabilities (Chateauneuf and Tallon 2002; Debreu and Koopmans 1982). Formally, for any $0 \leq \lambda \leq 1$ and acts $x, y, \lambda x+(1-\lambda) y$ is the act assigning outcome $\lambda x(s)+(1-\lambda) y(s)$ to each $s$. Quasi-concavity holds if $\lambda x+(1-\lambda) y \succcurlyeq x$ whenever $x \sim y$.

Theorem 5.5.8. Assume that all assumptions and results of Theorem 5.5.6 hold. Then the following four statements are equivalent.

(i) Utility is concave;

(ii) Risk aversion holds;

(iii) Quasi-concavity holds;

(iv) Diminishing rate of substitution holds.

In Theorem 5.5.8, Statement (ii) is less appealing for decision under uncertainty than the other conditions because this statement involves expected values and subjective probabilities, which are not directly observable. As regards DRS, each triple $\alpha^{i-1}, \alpha^{i}, \alpha^{i+1}$ in Eq. (5.4) entails a test of DRS, so that $n$ indifferences in Eq. (5.3) give $n-2$ tests of the condition. This illustrates that preference conditions in terms of $\sim^{*}$ are easily observable even if $\sim^{*}$ is a derived concept. DRS transparently corresponds with concavity of $U$ in Figure 5.2(b), where $\alpha^{j+1} \alpha^{j} \sim^{*} \alpha^{j} \alpha^{j-1}$ with $\alpha_{j}<\frac{\alpha^{j+1}+\alpha^{j-1}}{2}$ for all $j$.

Next, a comparative result is obtained using the $\sim^{*}$ relation. 
Theorem 5.5.9. Assume that for both $j=1$ and $j=2: \mathscr{A}^{j}$ is a subalgebra of $\mathscr{A}$; the outcome set is $\mathscr{C} \subset \mathbb{R}$; $\succcurlyeq^{j}$ is a preference relation over the acts that are measurable with respect to $\mathscr{A}^{j} ; \sim^{* j}$ refers to the corresponding tradeoff relation; all assumptions and results of Theorem 5.5.6 hold with algebra $\mathscr{A}^{j}$, subjective probability $P^{j}$ on $\mathscr{A}^{j}$, and utility function $U^{j}$. Then the following two statements are equivalent:

(i) $U^{1}=\varphi \circ U^{2}$ for a concave transformation $\varphi$;

(ii) $\succcurlyeq^{1}$ exhibits stronger DRS than $\succcurlyeq^{2}$.

The special case where $\succcurlyeq^{1}$ and $\succcurlyeq^{2}$ are preferences of the same decision maker and $\mathscr{A}^{1}$ and $\mathscr{A}^{2}$ are different sources, yields a within-person comparison similar to the Ellsberg two-color paradox. If $\succcurlyeq^{1}$ and $\succcurlyeq^{2}$ concern different different decision makers and $\mathscr{A}^{1}=\mathscr{A}^{2}$, then we have a between-person within-source comparison.

\subsection{Yaari's Comparative Risk Aversion Results}

It is well known that for decision under risk, decision maker 1 has a more concave utility function than decision maker 2, as in Statement (i) of Theorem 5.5.9, if and only if decision maker 1's risk premium (difference between expected value and certainty equivalent) exceeds that of decision maker 2 for every act. This condition is more difficult to handle for uncertainty for reasons as discussed above: subjective probabilities are not easily observable so that expected value and risk premium are so neither. Yaari (1969) showed that comparisons are still possible in terms of certainty equivalents (he used an equivalent formulation in terms of acceptance sets) under a restrictive condition. In other words, he showed equivalence of Statements (i) and (ii) below.

Theorem 5.6.1. Assume that for both $j=1$ and $j=2$ : the outcome set is the nondegenerate interval $\mathscr{C} \subset \mathbb{R} ; \succcurlyeq^{j}$ is a preference relation over all acts; all assumptions and results of Theorem 5.5.6 hold with algebra $\mathscr{A}^{j} \subset \mathscr{A}$, subjective probability $P^{j}$ on $\mathscr{A}^{j}$, and utility function $U^{j}$. Then the following three statements are equivalent:

(i) $U^{1}=\varphi \circ U^{2}$ for a concave transformation $\varphi$ and $P^{1}=P^{2}$;

(ii) For each act, the certainty equivalent for $\succcurlyeq^{2}$ is at least as large as that for $\succcurlyeq^{1}$; 
(iii) $\succcurlyeq^{1}$ exhibits stronger DRS than $\succcurlyeq^{2}$; further, $P^{1}=P^{2}$.

Under EU, probabilities are commonly taken to reflect beliefs, while utilities reflect attitudes towards risk. This separation is maintained in Theorem 5.5.9. In particular, Theorem 5.5.9 allows for comparison of risk attitudes irrespective of what beliefs are, so that identification of beliefs is no longer required. Yaari's (1969) result did not obtain such a separation of risk attitude and belief. As he showed, the risk attitudes of different decision makers could only be compared through his condition (ii) for the special case of identical beliefs.

\subsection{Recursive Models}

This section turns to recursive multi-stage models where in the first stage probabilities may be unknown but they are known in the second stage. Thus, all following models can be considered to be special cases of Anscombe and Aumann's (1963) model. $L$ denotes the set of lotteries, where lotteries (typically denoted by $\ell$ ) are probability distributions over $\mathscr{C}$. A typical act $x$ maps a state space $S$ to $L$, assigning a lottery $x(s)$ to each state $s .\left(E_{1}: \ell_{1}, \ldots, E_{m}\right.$ : $\ell_{m}$ ) denotes an act with $E_{1}, \ldots, E_{m}$ partitioning the state space $S$, and each $\ell_{i}, i=1, \ldots, m$, designating a lottery.

First-stage acts $x$ have their outcomes depend only on first-stage events, i.e., all lotteries $x(s)$ are degenerate. Second-stage acts $x$ have their outcomes depend only on the second-stage uncertainty, i.e. there exists a lottery $\ell$ such that $x(s)=\ell$ for all $s \subset S$. First-stage acts are identified with the corresponding mappings from $S$ to $\mathscr{C}$, and second-stage acts are identified with the lottery that they generate. The restrictions of the preference relation $\succcurlyeq$ to firstand second-stage acts are denoted $\succcurlyeq^{1}$ and $\succcurlyeq^{2}$. We will impose the richness assumptions of preceding sections on first- and second-stage acts. We do not need further richness assumptions. In particular, we need not assume the presence of every (measurable) allocation of lotteries to states $s$. This point will be crucial in the application to the model by Klibanoff, Marinacci, and Mukherji (2005). The following assumptions will be made sometimes, but not always.

Assumption 5.7.1 (Within-Source EU). $\succcurlyeq^{1}, P^{1}$, and $U^{1}$ satisfy all assumptions and results of Theorem 5.5.6. $\succcurlyeq^{2}, P^{2}$, and $U^{2}$ satisfy all assumptions and results of Corollary 5.5.7. 


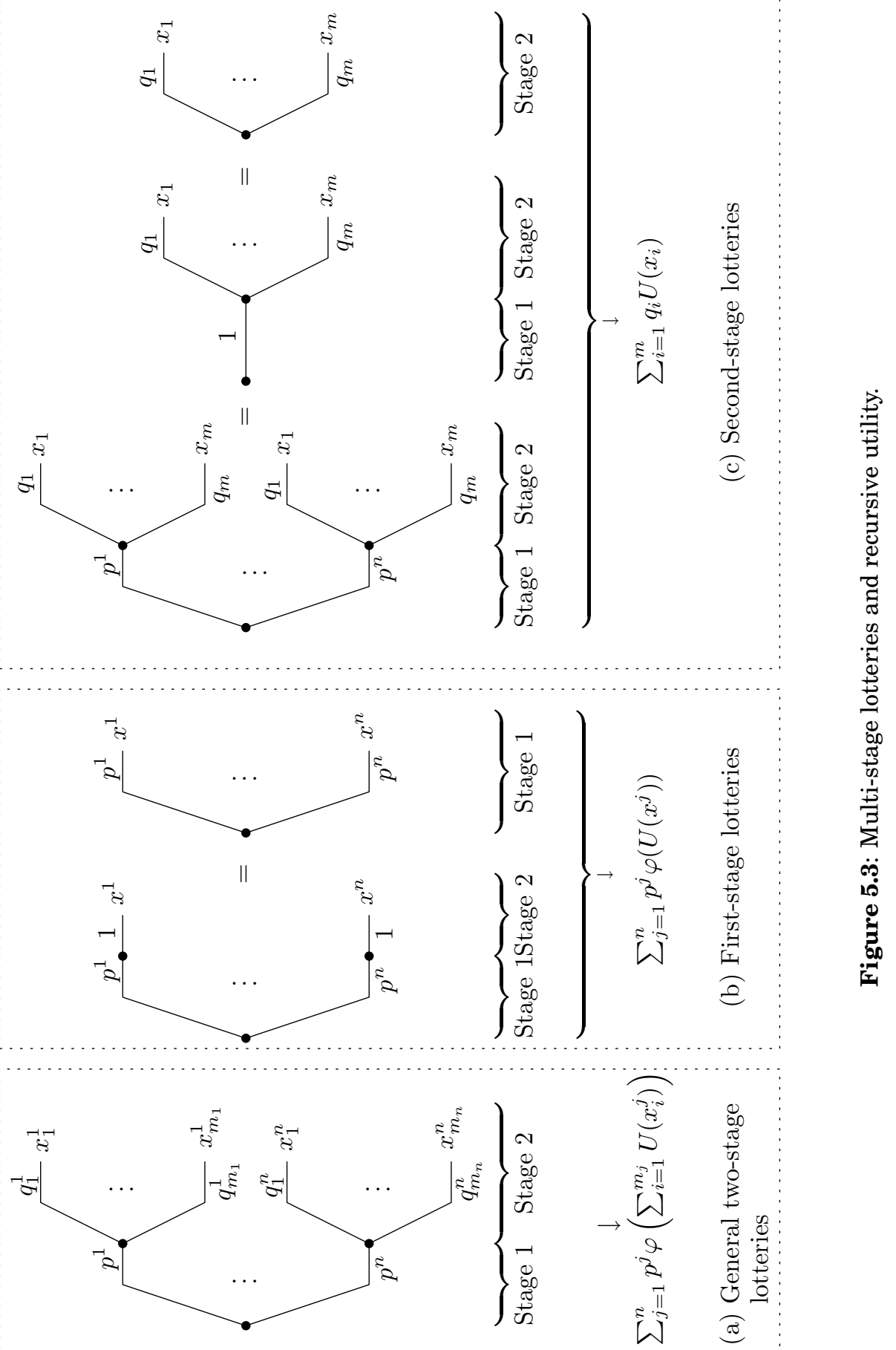


Assumption 5.7.2 (Backward Induction). The preference value of act $x$ is not affected if each lottery $x(s)$ is replaced by its $\succcurlyeq^{2}$ certainty equivalent, i.e. an outcome $\alpha_{s}$ with $\alpha_{s} \sim^{2} x(s)$.

By backward induction, a preference between any pair of acts can be derived from the preference between the first-stage acts that result after the substitutions of certainty equivalents. This implies, in particular, that $\succcurlyeq$ inherits weak ordering from $\succcurlyeq^{1}$. $E U^{2}$ denotes expected utility with respect to $U^{2}$ and the objectively given probabilities of stage 2 .

Theorem 5.7.3. Let the Structural Assumptions 5.4.1 and 5.4.2 hold for $\succcurlyeq^{1}$ and $\succcurlyeq^{2}$, respectively. Then the following two statements are equivalent.

(i) There exist continuous strictly increasing functions $U^{2}$ and $\varphi$, and a probability measure $P$ on $S$, such that acts are evaluated by

$$
x \rightarrow \int_{S} \varphi\left(E U^{2}(x(s))\right) d P(s) ;
$$

(ii) Assumption 5.7.2 holds and, for each $j=1,2, \succcurlyeq^{j}$ satisfies:

(a) weak ordering;

(b) monotonicity;

(c) continuity;

(d) truncation continuity;

(e) tradeoff consistency.

Statement (i) above means that Assumptions 5.7.1 and 5.7.2 hold. Several papers have studied conditions that imply concavity of $\varphi$ in the above theorem. They all need richness assumptions at least as strong as the following one.

Assumption 5.7.4 (Richness). Under the within-source EU Assumption 5.7.1, there exists an event $E \subset S$ with $0<P(E)=p<1$ such that for all $\alpha$ and $\beta$ in the image of $U^{2}$, there exists an act $\left(E: \ell_{1}\right.$, not- $\left.E: \ell_{2}\right)$ in the preference domain with $E U^{2}\left(\ell_{1}\right)=\alpha$ and $E U^{2}\left(\ell_{2}\right)=\beta$.

Preference for second-stage resolution (PSR) of uncertainty holds if, for each first-stage act $\alpha_{E} \beta$ and second-stage act $\alpha_{p} \beta$ with $P(E)=p, \alpha_{E} \beta \preccurlyeq$ 
$\alpha_{p} \beta$. In intertemporal contexts, preference for first-stage resolution of uncertainty is more common, although not universal. Nevertheless, to be consistent with some later results on ambiguity, where concavity rather than convexity of $\varphi$ is of interest, we analyze PSR and the implied concavity of $\varphi$ in our theorems. The analysis of preference for first-stage resolution of uncertainty is completely analogous, with the above preference reversed and with convexity of $\varphi$ rather than concavity.

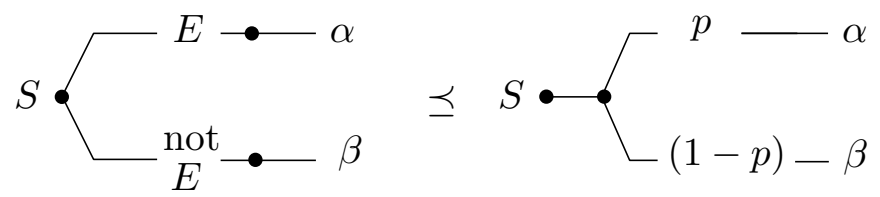

Figure 5.4: $P(E)=p$. Preference for second-stage resolution of uncertainty (PSR).

PSR can be interpreted as an aversion to mean-preserving spreads in terms of second-stage acts. The righthand act yields the second-stage mix $\alpha_{p} \beta$ with certainty. The lefthand act is a mean-preserving spread, yielding $\alpha$ at the second stage with probability $p$, and $\beta$ with probability $1-p$. Ergin and Gul (2009) considered a stronger condition, imposing aversion to meanpreserving spreads of second-stage acts in general. The present condition is equivalent to

$$
p \cdot \varphi\left(U^{2}(\alpha)\right)+(1-p) \cdot \varphi\left(U^{2}(\beta)\right) \leq \varphi\left(p \cdot U^{2}(\alpha)+(1-p) \cdot U^{2}(\beta)\right),
$$

which, given sufficient richness of $U^{2}$ 's image, is equivalent to concavity of $\varphi$ (see Lemma 5.A.1).

An alternative preference condition can be obtained if we use an alternativeoutcome interpretation, taking the EU values of the second-stage acts as outcomes. For an act $x$ we define the alternative-outcome act $x^{\prime}$ through $x^{\prime}(s)=E U^{2}(x(s))$ for all $s . x^{\prime}$ is a first-stage act. The expected value of $x$ under the alternative interpretation, i.e. the expected value of $x^{\prime}$ with respect to $P$ on $S$, is the preference value of the act $x$ that would result if $\varphi$ were the identity function. Klibanoff, Marinacci, and Mukherji (2005) henceforth abbreviated to $K M M$ - introduced smooth ambiguity aversion: any lottery $\ell$ with $E U(\ell)=E V\left(E U^{2}(x(s))\right)$ is preferred to the original act $x$. This condition amounts to traditional risk aversion imposed under the alternative-outcome interpretation. Given sufficient richness, the risk aversion mentioned is equivalent to concavity of $\varphi$. 
The two conditions just discussed, PSR and smooth ambiguity aversion, used the first-stage subjective probabilities as inputs, which makes their use in preference foundations problematic, as discussed before. The following condition does not have this problem.

For $0 \leq \lambda \leq 1$, and lotteries $\ell, \ell^{\prime}, \lambda \ell+(1-\lambda) \ell^{\prime}$ denotes a probabilistic mixture defined in the usual way. For acts $x, y$, and $0 \leq \lambda \leq 1$, the probabilistic mixture of acts $\lambda x+(1-\lambda) y$ is defined through statewise probabilistic mixing, assigning lottery $\lambda x(s)+(1-\lambda) y(s)$ to each $s$. Given EU preferences over second-stage acts, the probabilistic mixture of acts just defined, is equivalent to outcome mixing under the alternative-outcome interpretation. Gilboa and Schmeidler (1989) and Schmeidler (1989) defined uncertainty aversion: $\lambda x+(1-\lambda) y \succcurlyeq x$ whenever $x \sim y$. The condition is equivalent to quasi-concavity under the alternative-outcome interpretation. We now summarize various ways to get concavity of $\varphi$.

Theorem 5.7.5. Assume the conditions and assumptions of Theorem 5.5.9 for $\succcurlyeq^{1}$ and $\succcurlyeq^{2}$. The following two statements are equivalent:

(i) $\varphi$ is concave;

(ii) $\succcurlyeq^{1}$ exhibits stronger DRS than $\succcurlyeq^{2}$.

If we further have Assumptions 5.7.2 and 5.7.4, then the following three statements are also equivalent to the above two statements:

(iii) Smooth ambiguity aversion holds;

(iv) PSR (preference for second-stage resolution of uncertainty) holds;

(v) Uncertainty aversion holds.

In the above theorem, the condition in (ii) is more generally applicable than those in (iii), (iv), and (v). It shares with condition (v) the advantage over the conditions (iii) and (iv) that it does not need subjective first-stage probabilities as inputs, which is important if the first-stage probabilities are not objectively given.

\subsection{Comparative Results for Recursive Models}

We now turn to comparative results. KMM proposed the following condition. It is only defined for decision makers $\succcurlyeq^{A}$ and $\succcurlyeq^{B}$ with same first-stage 
beliefs $P^{A}=P^{B}$; then $\succcurlyeq^{A}$ is more smooth-ambiguity averse than decision maker $\succcurlyeq^{B}$ if

$$
x \succcurlyeq^{A} \ell \Rightarrow x \succcurlyeq^{B} \ell
$$

for all acts $x$ and second-stage acts $\ell$. As pointed out by KMM, this condition implies that $\succcurlyeq^{A}$ and $\succcurlyeq^{B}$ are identical on lotteries, as follows by substituting lotteries for $x$. Although for general preference relations the resulting condition need not imply identical preferences ${ }^{1}$, it does so for nondegenerate preferences that maximize EU, such as $\succcurlyeq^{2 A}$ and $\succcurlyeq^{2 B}$. Given the same preferences over lotteries, we may restrict Eq. (5.5) to degenerate lotteries $\ell$, i.e. sure outcomes, these being the same for $\succcurlyeq^{A}$ and $\succcurlyeq^{B}$. Thus, comparative smooth ambiguity aversion amounts to the requirement of identical preferences over lotteries plus Yaari's certainty-equivalent condition (ii) in Theorem 5.6.1.

Theorem 5.8.1. For both $\succcurlyeq^{A}$ and $\succcurlyeq^{B}$, assume the conditions and assumptions of Theorem 5.7.3, with the notation $\succcurlyeq^{1 A}, \succcurlyeq^{2 A}, \succcurlyeq^{1 B}$, and $\succcurlyeq^{2 B}$ as before. The following two statements are equivalent:

(i) $\varphi^{A}$ is a concave transformation of $\varphi^{B}$ and $U^{2 A}=U^{2 B}$;

(ii) $\succcurlyeq^{1 A}$ exhibits stronger DRS than $\succcurlyeq^{1 B}$; further $\sim^{* 2 A}=\sim^{* 2 B}$.

If we further have Assumptions 2.5.2 and 2.5.4 for both $\succcurlyeq^{A}$ and $\succcurlyeq^{B}$, and if $P^{A}=P^{B}$, then the following statement is also equivalent to the above two statements:

(iii) $A$ is more smooth-ambiguity averse than $B$.

We are not aware of similar comparative results using uncertainty aversion. The above results compared ambiguity attitudes only under the restrictive assumption of identical preferences over lotteries. We next show how ambiguity attitude can be compared without this restriction.

Theorem 5.8.2. For both $\succcurlyeq^{A}$ and $\succcurlyeq^{B}$, assume the conditions and assumptions of Theorem 5.7.3, with the notation $\succcurlyeq^{1 A}, \succcurlyeq^{2 A}, \succcurlyeq^{1 B}$, and $\succcurlyeq^{2 B}$ as before. The following two statements are equivalent:

(i) $\varphi^{A}$ is a concave transformation of $\varphi^{B}$;

\footnotetext{
${ }^{1}$ Loosely speaking, $\succcurlyeq^{2 B}$ might have more indifferences than $\succcurlyeq^{2 A}$.
} 
(ii) If $\alpha \beta \sim^{* 1 B} \beta \gamma$ and $\alpha \beta^{\prime} \sim^{* 1 A} \beta^{\prime} \gamma$, then $\beta^{\prime} \leq \bar{\beta}$ whenever $\bar{\beta} \sim^{2 A} \alpha_{p} \gamma$ and $\beta \sim^{2 B} \alpha_{p} \gamma$ for some $p .{ }^{2}$

The condition $\bar{\beta} \sim^{2 A} \alpha_{p} \gamma$ and $\beta \sim^{2 B} \alpha_{p} \gamma$ in statement (ii) ensures that $\bar{\beta}$ has the same position relative to $\alpha$ and $\gamma$ in terms of $U^{2 A}$ as $\beta$ has in terms of $U^{2 B}$. In other words, Statement (ii) in Theorem 5.8.2 is Definition 5.5.2 ("Stronger DRS") reformulated for the alternative-outcome interpretation. In Theorem 5.8.2, identical preferences over lotteries can be imposed by adding the requirement of $\sim^{* A}=\sim^{* B}$, or by requiring that always $\beta=\bar{\beta}$ in Condition (ii). Thus, the restriction of identical preferences under risk is optional in Theorem 5.8.2.

\subsection{Alternative Recursive Models}

We show how the above results generalize a number of classical multi-stage results in the literature. All of these models make the within-source EU Assumption 5.7.1 and we will maintain this assumption in the following discussion. The first study that used a representation as in Statement (i) of Theorem 5.7.3 was Kreps and Porteus (1978) (they only considered bounded utility). They obtained the equivalence of Statements (i) and (iv) in Theorem 5.7.5. Their result was applied to intertemporal decision making, assuming that the first-stage uncertainty was resolved before the second-stage uncertainty. Then preference for first-stage resolution amounts to a preference for an early resolution of uncertainty. Statement (ii) in Theorem 5.7.5 is the equivalence resulting from our Theorem 5.5.6. Section 5.8 has provided comparative extensions of Kreps and Porteus' results, where one decision maker has a stronger preference for late (or early) resolution of uncertainty than another decision maker. Under Assumptions 5.7.1 and 5.7.2, Statement (ii) can be used as an alternative to the condition of Kreps and Porteus. It, however, extends to cases where Assumptions 5.7.1 and 5.7.2 need not hold.

For nonexpected utility it is desirable that Assumption 5.7.1, necessitating a violation of the reduction of compound lotteries, be relaxed. It is well known that one of some desirable dynamic-decision principles has to be abandoned under nonexpected utility (Hammond 1988). Machina (1989)

\footnotetext{
${ }^{2}$ There always exists a probability $p$ with $\beta \sim^{2 B} \alpha_{p} \gamma$, because, by Lemma 5.5.3, $U^{2 B}(\beta)$ is between $U^{2 B}(\alpha)$ and $U^{2 B}(\gamma)$, and (excluding the trivial case where $U^{2 B}(\alpha)=U^{2 B}(\gamma)$, when $\beta=\bar{\beta}=\gamma$, and $p$ can be anything) $p$ is uniquely determined by $U^{2 B}(\beta)=p \cdot U^{2 B}(\alpha)+(1-$ $p) \cdot U^{2 B}(\gamma)$. By continuity of $U^{2 B}$ there exists a $\bar{\beta}$. This $\bar{\beta}$ is unique because $U^{2 B}$ is strictly increasing.
} 
strongly argued for abandoning consequentialism rather than the reduction of compound lotteries or dynamic consistency. Karni and Safra (1989) argued for abandoning, in modern terminology, dynamic consistency. Our analysis in terms of $\sim^{*}$ leaves all these options open, and does not commit to the violation of reduction of compound lotteries.

Kreps and Porteus (1978) considered multi-stage models; their results follow from repeated application of the two-stage results. They formulated their result for the special case of decision under risk, where the probability measure $P$ in the first-stage is also objective and given beforehand, so that it can easily be used as input for obtaining testable preference conditions. For decision under uncertainty, where $P$ is subjective and has to be derived from choice in the revealed preference approach, their condition of PSR is not easy to observe. Then an equality $P(E)=p$ can only be derived from observed choice, which need not always be easy to do. For exampe, if $S=\left\{s_{1}, s_{2}\right\}$ and $P\left(s_{1}\right)=\sqrt{0.5}$, then we are not aware of a finite number of observed preferences within the model assumed that can reveal this probability ${ }^{3}$. Our conditions are directly observable even if first-stage probabilities are subjective.

Neilson (1993) (brought to our attention by a reference in KMM) presented the equivalence of Statements (i) and (iii) in Theorem 5.7.5. To obtain the representation in Theorem 5.7.3, he imposed the von NeumannMorgenstern axioms on lotteries, and Savage's (1954) axioms on first-stage acts, where he added a weak continuity axiom to imply continuity of utility; it also implies boundedness of utility. He applied his result to ambiguity, with the first-stage events ambiguous. Then smooth ambiguity aversion and concavity of $\varphi$ can be interpreted as ambiguity aversion. In this approach the first-stage probabilities $P$ are not objective or known but are subjective and must be derived from choice. Then the first-stage probabilities $P$ in smooth ambiguity aversion are not easy to observe, which makes smooth ambiguity aversion not so easy to observe empirically. This problem is similar to the problem of PSR. In addition, smooth ambiguity aversion uses the utility function $U^{2}$, which must similarly be derived from preference ${ }^{4}$. We

\footnotetext{
${ }^{3}$ We recall that the objective probabilities of the second stage involve different utilities so that they cannot be used for calibration purposes.

${ }^{4}$ This problem can be mitigated. Assume that there are a maximal outcome $M$ and a minimal outcome $m$, and normalize $U^{2}(M)=1$ and $U^{2}(m)=0$. We can replace every secondstage act by the act $\left(p: M, 1-p: m\right.$ ) equivalent to it, and the $E U^{2}$ value of that secondstage act then is $p$, which makes $E U^{2}$ relatively easy to observe so that it can be used in behavioral preference axioms. In this manner, utilities are converted into probabilities and
} 
have relaxed these restrictions and have added comparative results.

Theorem 5.7.3 provides a complete preference foundation for the basic decision model of $\mathrm{KMM}^{5}$. These authors indicated that such a foundation was possible but for brevity did not elaborate on it. We can present the entire preference foundation and at the same time maintain brevity because we use the same $\sim^{*}$ tool in all our results. Whereas in KMM's model utility must be bounded for each act, we also allow for acts with unbounded utility.

KMM obtained the equivalence of Statements (i) and (iii) in Theorem 5.7.5. A major step forward in KMM's analysis was that through subtle interpretations of the concepts involved they opened a new way to analyze ambiguity. Their interpretations are discussed in some detail. At the outset, the uncertainty in the second stage in their model concerns a Savagean state space $T$ rather than given probabilities as in our approach. The uncertainty in the first stage concerns what is the appropriate subjective second-stage probability measure to use for the Savage state space; this uncertainty entails a psychologically realistic modeling of ambiguity. Thus, in our notation, every state $s \in S$ specifies what the subjective probability measure over $T$ is. First-stage acts (called second-order acts in their model) assign outcomes contingent on what the appropriate second-stage subjective probability measure on $T$ is. KMM assume that the two-stage decomposition is endogenous. By nevertheless assuming preferences between first-stage acts to be available, they make it possible to have this endogenous two-stage decomposition observable. Thus, their model becomes considerably more broadly applicable while at the same time coming close to our psychological perceptions of ambiguity. By assuming the second-stage probabilities over $T$ to be completely specified by the first-stage states, KMM achieve that subjective probabilities over the state space $T$ can be treated as known probabilities in the second stage (KMM, Lemma 1, Definition 2, and Assumption 3). This leads to the paradoxical but extremely useful result that the Savagean secondstage uncertainty can be treated as objective risk, as in the second stage of the Anscombe-Aumann model as we do. All aspects of ambiguity are controled in the first stage. The following remark prepares for explaining our generalization of the required richness of KMM's model.

Remark 5.9.1 (Richness in KMM). Not all mappings from $S$ to $L$ need to be

smooth ambiguity aversion, i.e. risk aversion with respect to second-stage EU, can be seen to be stronger than a preference for first-stage resolution of uncertainty.

${ }^{5}$ These authors assumed countably additive probability measures. This condition can be ensured by adding set-continuity as in Theorem 5.5.6. 
available in the preference domain of KMM. They do assume that all firststage acts (called second-order acts) are available. They also assume that there is a subalgebra of events on $T$ that have objective probabilities (being the same under all second-stage probability measures $P^{2}$ considered) and that is rich enough to generate all probability distributions over outcomes, so that there is also richness for the second-stage acts. They, further, assume that $T=\Omega \times(0,1]$ where the second component $(0,1]$ generates these objective probabilities, leading to an Anscombe- Aumann-like two-stage decomposition of $T$. This composition only serves to calibrate risk attitude and should not be confused with our Anscombe-Aumann decomposition. The second stage of our decomposition concerns the whole space $T$.

Using Assumption 5.7.2, we can generate the preference values of all mappings from $S$ to $L$ in the KMM model. KMM impose some richness assumptions on the set of acts, i.e. two-stage acts with nondegenerate Savagean acts/lotteries assigned to first-stage states of nature, with thought experiments involving two different first-stage subjective probability measures with disjoint supports. In this manner, they derive the richness of Assumption 5.7.4 in the proof of their Proposition 1.

The contribution of Theorem 5.5.8 (Statement (ii) in Theorem 5.7.5) to the ambiguity-aversion characterization of KMM is that it, again, avoids the need to use subjective probabilities or second-stage utilities as inputs in a preference condition, and that it does not need Assumptions 5.7.1 and 5.7.2. All it needs is that all simple first-stage acts ("second-order acts") are available, and those second-stage acts in the model of KMM that have their outcomes depend only on events with known probabilities (called lotteries by KMM). In particular, we do not impose any richness on the objects of primary interest in the KMM model, i.e. the mappings from $\Omega$ to outcomes (i.e. Savagean acts). We do not need the richness assumptions in the second paragraph of Remark 5.9.1. Thus, we leave complete flexibility regarding the important Savagean acts. Also, we require no commitment to a violation of reduction of compound lotteries, and no boundedness of the acts. We hope that these generalizations can enhance the applicability of KMM's approach to ambiguity.

We also generalized KMM's results on comparative ambiguity aversion. We did not use the subjective first-stage probabilities as inputs and, in particular, need not assume that these are the same for different decision makers. We also did not require same preferences over lotteries. Thus, ambiguity attitude can be analyzed as a component completely independent from 
beliefs and risk attitudes.

Nau (2006) assumed the two-stage decomposition of Figure 5.4 to be exogenous. He considered a state-dependent generalization of EU for firstand second-stage acts, and then used a local version of PSR (called local uncertainty aversion) to characterize concavity of his analog of $\varphi$. He also expressed this condition in terms of generalized Pratt-Arrow measures. Statedependent versions of our results could be obtained by using event-dependent relations $\sim_{E}^{*}$ in Eqs. (5.1) and (5.2), but we will not pursue this point here. Grant, Kajii, and Polak (2001) used a two-stage model as in theorem 5.7.3 in a game-theory context.

\subsection{Discussion}

We consider the intuitive objection against the use of marginal utility for analyzing risk and ambiguity, raised before, from a different perspective. it may be argued that the DRS condition used in Theorem 5.5.8 is not as appealing as the traditional definition of risk aversion because the former does not speak to risk or probability in any direct manner, whereas the latter clearly does. We agree that these two conditions are intuitively different. This difference can be interpreted as an argument against EU. Advocates of EU have to consider the conditions as equivalent, simply as a logical fact.

Similar observations apply to our theorems. In Theorem 5.6.1, even if $P^{1}=P^{2}$, then still stronger DRS (condition iii) does not speak to risk as Yaari's certainty-equivalent condition (in ii) does. In Theorem 5.7.5 on recursive utility, stronger DRS (in ii) does not speak to ambiguity as smooth ambiguity aversion and the other conditions do, with similar observations for the comparative results in Theorem 5.8.2. When these results are interpreted in the intertemporal sense of Kreps \& Porteus, stronger DRS does not even consider the intertemporal structure (with the advantage of not having to use acts with outcomes depending on more than one stage), much as it does not even consider the probabilities or ambiguous events. In all these cases, the EU assumptions as used in the models mentioned would imply that our DRS conditions are equivalent, which turns against these EU assumptions. The criticism of EU advanced here can be compared to Rabin's (2000) criticism of EU. He derived unacceptable implications of taking utility as an index of risk attitude. This chapter puts forth undesirable implications of EU when utility is used to model ambiguity. 


\subsection{Concluding Remarks}

This chapter presented a convenient tool for analyzing marginal utility. It entails many generalizations of classical results and alternative preference foundations and interpretations. Readers who have doubts about the appropriateness of EU to model risk and ambiguity attitudes can test their confidence in EU by inspecting if the alternative preference conditions put forward in this chapter can convey the same intuition as preceding preference conditions used in the literature. If they do, then EU is appropriate. If they do not, then EU must be questioned. For example, if the reader expects that smooth ambiguity aversion is found empirically in the two-stage model of section 5.7, but that the standard sequences in Eq. (5.4) will be the same and will exhibit the same utility graphs (Fig. 5.2(b)) for stage 1 and stage 2 uncertainty, then this amounts to EU not being valid.

\section{A Appendix: Proofs}

We begin with two lemmas that will be useful for the elaboration of our main results.

Lemma 5.A.1. Let $f: I \rightarrow \mathbb{R}$ be continuous, with $I \subset \mathbb{R}$ an interval. Then $f$ is concave if and only if, for every $\alpha, \beta \in I$, there exists a $p_{\alpha, \beta}$ with $0<p_{\alpha, \beta}<1$ and $f\left(p_{\alpha, \beta} \cdot \alpha+\left(1-p_{\alpha, \beta}\right) \cdot \beta\right) \geq p_{\alpha, \beta} \cdot f(\alpha)+\left(1-p_{\alpha, \beta}\right) \cdot f(\beta)$.

Proof. This follows from Hardy, Littlewood, and Pòlya (1934, Observation 88). We will only need the case where $p_{\alpha, \beta}=p$ is independent of $\alpha, \beta$.

Lemma 5.A.2. Let $I \subset \mathbb{R}$ be an interval. A continuous and strictly increasing function $f: I \rightarrow \mathbb{R}$ is concave if and only if $\left[f(\alpha)-f(\beta)=f(\beta)-f(\gamma) \Rightarrow \beta \leq \frac{\alpha+\gamma}{2}\right]$.

Proof. The condition between brackets is equivalent to midpoint concavity, which is the case of Lemma 5.A. 1 with $p_{\alpha, \beta}=0.5$ for all $\alpha, \beta$, and is equivalent to concavity of $f$ by Lemma 5.A.1.

Proof of Lemma 5.5.3. See Wakker (2009).

Proof of Theorem 5.5.6. 
[(i) $\Rightarrow$ (ii)]. This follows from substitution. For simple acts and conditions (a) - (c) and (e), it was established by Köbberling and Wakker (2003, Corollary 10). For general acts and condition (e), it was established by Wakker (1993, Lemma 1.8, Corollary 2.14, and Section 4.4).

[(ii) $\Rightarrow$ (i)]. Assume (ii). We first restrict attention to simple acts, for which we will not use truncation continuity. For finite state spaces the result was obtained by Köbberling and Wakker (2003). The extension to all simple acts for a general state space is routine.

The extension of the representation to general, possibly unbounded, acts follows from Theorem 2.5 in Wakker (1993). Note here that EU is a special case of Wakker's CEU (Choquet expected utility), and that Wakker's step equivalent assumption is implied by the existence of certainty equivalence.

[Further Results]. The uniqueness results follow from Köbberling and Wakker (2003, Corollary 10). The set-continuity, necessary and sufficient for countable additivity, follows from substitution similar to Wakker (1993, Section 4.1; for additive measures we only need Wakker's (1993) Eq. 4.2, and only for $A=\varnothing$.).

Proof of Theorem 5.5.8. (i) $\Rightarrow$ (ii) is exactly as for decision under risk.

For (ii) $\Rightarrow$ (i) assume (ii). By nondegeneracy, there exists an event $E$ with $0<P(E)=p<1$. Risk aversion implies $\alpha_{E} \beta \preccurlyeq(p \cdot \alpha+(1-p) \cdot \beta$ ) (the latter taken as degenerate) and thus, under EU, $U(p \alpha+(1-p) \beta) \geq p \cdot U(\alpha)+(1-$ $p) \cdot U(\beta)$. By Lemma 5.A.1, $U$ is concave.

(i) $\Leftrightarrow$ (iii) is due to Debreu and Koopmans (1982).

(i) $\Leftrightarrow$ (iv) follws from Lemmas 5.5.3 and 5.A.2.

Proof of Theorem 5.5.9. Express outcomes in $U^{1}$ units, and apply Theorem 5.5.8 to $\varphi$ instead of $U$ with $\varphi$ such that $U^{2}=\varphi \circ U^{1}$.

Proof of Theorem 5.6.1. (i) $\Leftrightarrow$ (ii) is by Yaari (1969). (i) $\Leftrightarrow$ (iii) is by Theorem 5.5.9, with equality of probabilities added.

Proof of Theorem 5.7.3. $\quad$ For (i) $\Rightarrow$ (ii), assume (i). EU with $P^{j}$ and $U^{j}$ represents $\succcurlyeq^{j}$ for $j=1$ (because $\varphi$ is strictly increasing) and 2 (with $U^{1}=$ $\varphi \circ U^{2}$ ), which by Theorem 5.5.6 implies conditions (a) - (d) in Statement (ii). Assumption 5.7.2 follows because all $\alpha^{i}$ have the same $E U^{2}$ value as the acts they replace.

We now assume (ii) and derive (i). Theorem 5.5.6 applied to both $\succcurlyeq^{1}$ and $\succcurlyeq^{2}$ implies that there exist continuous and strictly increasing functions 
$U^{1}$ and $U^{2}$ and a probability measure $P$ such that the first-stage acts $\left(E_{1}^{1}\right.$ : $\left.x_{11}, \ldots, E_{m}^{1}: x_{m 1}\right)$ are evaluated by

$$
\sum_{i=1}^{m} P\left(E_{i}\right) U^{1}\left(x_{i 1}\right)
$$

and the second-stage acts are evaluated by $E U^{2}$, being EU with respect to $U^{2}$. Define the continuous strictly increasing $\varphi^{E}=U^{1} \circ\left(U^{2}\right)^{i n v}$.

Consider any act $x$. By continuity and strict increasingness of $U^{2}$, we can obtain $\alpha_{s}$ such that $\alpha_{s} \sim^{2} x(s)$ (i.e., these have the same $E U^{2}$ value) for all $s$. By backward induction, $x \sim y$ with $y(s)=\alpha_{s}$ for all $s$. Thus, the evaluation of $x$ must equal:

$$
\int_{S} U^{1}(y(s)) d P(s)=\int_{S} \varphi\left(U^{2}(y(s))\right) d P(s)=\int_{S} \varphi\left(E U^{2}(x(s))\right) d P(s) .
$$

Proof of Theorem 5.7.5. (i) $\Leftrightarrow$ (ii) is by Theorem 5.5.9.

(i) $\Leftrightarrow$ (iii) is due to KMM (Proposition 1). In our setup, the derivation is as follows. (i) $\Rightarrow$ (iii) is the traditional risk aversion implication. For (iii) $\Rightarrow$ (i), smooth ambiguity aversion applied to event $E$ from Assumption 5.7.4 implies that $\varphi(p \cdot \alpha+(1-p) \cdot \beta) \geq \varphi(\alpha)+(1-p) \cdot \varphi(\beta)$ which, by Lemma 5.A.1, implies (i).

For (i) $\Leftrightarrow$ (iv), make Assumption 5.7.2, and assume that $0<P(E)=p<1$ for some first-stage event $E$. Take some arbitrary $U^{2}(\alpha)$ and $U^{2}(\beta)$. Then $\alpha_{E} \beta \preccurlyeq \alpha_{p} \beta$ implies $p \cdot \varphi\left(U^{2}(\alpha)\right)+(1-p) \cdot \varphi\left(U^{2}(\beta)\right) \leq \varphi\left(p \cdot U^{2}(\beta)+(1-p) \cdot U^{2}(\beta)\right)$. By Lemma 5.A.1, $\varphi$ is concave.

(i) $\Leftrightarrow$ (v) follows from the equivalence (i) $\Leftrightarrow$ (iii) of Theorem 5.5.8 under the alternative-outcome interpretation.

Proof of Theorem 5.8.1. For (i) $\Leftrightarrow$ (ii), we note that $\sim^{* 2 A}=\sim^{* 2 B}$ is equivalent to $U^{2 A}$ and $U^{2 B}$ having same equalities of utility differences, which, given that they are defined on an interval and are continuous and strictly increasing, is equivalent to them being the same in the sense of being in the same interval class.

By Theorem 5.5.8, the first part of condition (ii) is equivalent to $U^{1 A}$ being more concave than $U^{1 B}$. Because $U^{1 A}=\varphi^{A} \circ U^{2 A}, U^{1 B}=\varphi^{B} \circ U^{2 B}$ and $U^{2 A}=U^{2 B}$, it is equivalent to $\varphi^{A}$ being more concave than $\varphi^{B}$.

(i) $\Leftrightarrow$ (iii) is due to KMM (Theorem 2), and follows from the equivalence of (i) and (ii) in Theorem 5.4.1 under the alternative-outcome interpretation. 
Proof of Theorem 5.8.2. First, $\varphi^{A}$ and $\varphi^{B}$ being continuous and strictly increasing functions, there exist a continuous and strictly increasing function $\psi$ such that $\varphi^{A}=\psi \circ \varphi^{B}$. Let us fix $U^{2 A}(\alpha)=U^{2 B}(\alpha)=a, U^{2 A}(\gamma)=U^{2 B}(\gamma)=c$ and $U^{2 A}\left(\beta^{\prime}\right)=b^{\prime}$. Then $\bar{\beta} \sim^{2 A} \alpha_{p} \gamma$ and $\beta \sim^{2 A} \alpha_{p} \gamma$ imply $U^{2 B}(\beta)=U^{2 A}(\bar{\beta})=b$ (where $b=p \cdot a+(1 . p) \cdot c$ ). Statement (ii) can be rewritten: $\varphi^{B}(a)-\varphi^{B}(b)=$ $\varphi^{B}(b)-\varphi^{B}(c)$ and $\psi \circ \varphi^{B}(a)-\psi \circ \varphi^{B}\left(b^{\prime}\right)=\psi \circ \varphi^{B}\left(b^{\prime}\right)-\psi \circ \varphi^{B}(c)$ imply $\varphi^{B}\left(b^{\prime}\right) \leq$ $\varphi^{B}(b)$. By Lemma 5.A.2, $\psi$ must be concave. The theorem follows. 


\section{Chapter 6}

\section{Continuous Fictitious Play in Zero-Sum Games}

\subsection{Introduction}

Fictitious Play is a procedure in which at each instance, players of a game myopically play their best replies against the opponents' past play. The origins of Fictitious Play lie in a series of papers by Brown $(1949,1951)$ and Robinson (1951). While today, the algorithm is usually interpreted as a myopic learning process, the initial objective of these studies was to develop an easy method to find the value of zero-sum games. In his 1949 article, Brown already argued heuristically that the continuous version of the procedure - Continuous Fictitious Play (CFP) - must converge at a linear speed to the set of Nash equilibria, and thus, the value of the game. This result is again mentioned - without proof - in Brown (1951). Brown's to a large extent heuristic approach towards CFP may be explained by the fact that he was primarily interested in a discrete algorithm, and thus, only considered convergence of CFP in zero-sum games as support for his conjecture that Discrete Fictitious Play (DFP) in zero-sum games converges to equilibrium.

The proof of this conjecture was obtained by Robinson (1951) in the early fifties. However, it was not until Hofbauer (1994) and Harris (1998) that the result for CFP was rigorously proven. Today, Harris' proof has become the standard reference. Essentially, he shows that the sum of players' instantaneous improvement steps in a zero-sum game, is a Lyapunov function. While this argument appears to be quite simple at first glance, it requires several 
rather technical lemmas ${ }^{1}$. Moreover, it makes use of nontrivial results on dynamical systems. Different versions of Harris' proof - often in a simplified form - have appeared in the literature. See a.o. Krishna and Sjöström (1997), Shamma and Arslan (2004), and Hofbauer and Sorin (2006).

In this chapter we provide a simple alternative argument. We show that the convergence of CFP in two-player zero-sum games follows directly from a result obtained by Monderer et al. (1997) that says that for CFP in any game, each player's instantaneous expected payoff will in the long run coincide with the average payoff that player has realized so far. Using the same approach, convergence of DFP is established up to the condition of infrequent switching (Fudenberg and Levine 1995; Monderer et al. 1997). That is, if for DFP in a zero-sum game players play each pure strategy profile for increasingly long periods of time, then the DFP converges to equilibrium. Simulations lead us to conjecture that infrequent switching is the engine behind Robinson's result ${ }^{2}$. Whether DFP in zero-sum games satisfies the condition of infrequent switching in general, remains an open question.

\subsection{Preliminaries}

\subsubsection{Zero-sum Games}

A zero-sum game is represented as an $m \times n$ matrix $A$. There are two players - 1 and 2. Player $1[2]$ has a pure strategy set $I[J]$ of cardinality $m[n]$. If player 1 plays strategy $i$ and 2 plays $j$, then player 1 receives a payoff of $a_{i j}$ and 2 of $-a_{i j}$. Let $\Delta^{m}$ and $\Delta^{n}$ be the sets of probability distributions over sets of respectively $m$ and $n$ alternatives; $\Delta^{m}$ and $\Delta^{n}$ are players' respective sets of mixed strategies over $I$ and $J$. A pure strategy $i \in I$ is also denoted as the unit vector $e^{i} \in \Delta^{m}$; we write $\tilde{I}:=\left\{e^{i} \mid i \in I\right\}$. Analogously, we denote player 2's $j$-th pure strategy also as the $j$-th unit vector $f^{j} \in \Delta^{n}$, and $\tilde{J}:=\left\{f^{j} \mid j \in J\right\}$. Let $\beta_{1}: \Delta^{n} \rightarrow \Delta^{m}$ be player 1's best-reply correspondence. That is,

$$
\beta_{1}(\hat{q}):=\left\{p \in \Delta^{m} \mid p A \hat{q} \geq p^{\prime} A \hat{q} \text { for all } p^{\prime} \in \Delta^{m}\right\} .
$$

Player 2's best-reply correspondence, $\beta_{2}: \Delta^{m} \rightarrow \Delta^{n}$ is defined as

$$
\beta_{2}(\hat{p}):=\left\{q \in \Delta^{n} \mid \hat{p} A q \leq \hat{p} A q^{\prime} \text { for all } q^{\prime} \in \Delta^{n}\right\} .
$$

\footnotetext{
${ }^{1}$ See also Hofbauer and Sorin (2006).

${ }^{2}$ Note that convergence of CFP implies convergence of DFP for a class of games that includes zero-sum games. See Harris (1998) and Hofbauer and Sorin (2006).
} 
Define $\beta: \Delta^{m} \times \Delta^{n} \rightarrow \Delta^{m} \times \Delta^{n}$ as $\beta(p, q):=\left(\beta_{1}(q), \beta_{2}(p)\right)$. The set of Nash equilibria of a game $A$ is defined as

$$
N E(A):=\left\{(p, q) \in \Delta^{m} \times \Delta^{n} \mid(p, q) \in \beta(p, q)\right\} .
$$

That is, $N E(A)$ is the set of fixed points of the best-reply correspondence $\beta$. Define the function $W: \Delta^{m} \times \Delta^{n} \rightarrow \mathbb{R}$ as

$$
W(\hat{p}, \hat{q}):=H(\hat{q})-L(\hat{p}),
$$

where $H(\hat{q}):=\max _{p \in \Delta^{m}} p A \hat{q}$ and $L(\hat{p}):=\min _{q \in \Delta^{n}} \hat{p} A q$.

Theorem 6.2.1. $W(p, q) \geq 0$, with equality if and only if $(p, q) \in N E(A)$.

This is a classic result that follows from von Neumann's (1928) maximin theorem. For an exact proof, see a.o. Myerson (1991).

\subsubsection{Continuous Fictitious Play}

A Continuous Fictitious Play (CFP) in $A$ is a pair $(x(t), y(t))$ of Lebesgue measurable functions $x:[0, \infty) \rightarrow \tilde{I}$ and $y:[0, \infty) \rightarrow \tilde{J}$, such that for almost all $t \geq 1$, we have

$$
(x(t), y(t)) \in \beta\left(\frac{1}{t} \int_{0}^{t} x(\tau) d \tau, \frac{1}{t} \int_{0}^{t} y(\tau) d \tau\right) .
$$

Players' beliefs $(p(t), q(t))$ are defined as functions

$$
p(t):=\frac{1}{t} \int_{0}^{t} x(\tau) d \tau, \text { and } q(t):=\frac{1}{t} \int_{0}^{t} y(\tau) d \tau
$$

on the domain $[1, \infty)$. Furthermore, we define the set $\Omega$ of limit points of $(p(t), q(t))$ as

$$
\Omega:=\bigcap_{T \geq 1} \operatorname{cl}(\{(p(t), q(t)) \mid t \geq T\}),
$$

where cl denotes the topological closure. We say $(p, q)$ converges to Nash equilibrium if $\Omega \subseteq N E(A, B)$. Expected and average payoffs are defined for $t \in[1, \infty)$ as

$$
E_{1}(t):=x(t) A q(t) \text {, and } E_{2}(t):=-p(t) A y(t),
$$


and

$$
P_{1}(t):=\frac{1}{t} \int_{0}^{t} x(\tau) A y(\tau) d \tau \text {, and } P_{2}(t):=-P_{1}(t) .
$$

Monderer et al. (1997) introduce the concept of belief affirmation; a CFP is said to be belief affirming if

$$
\lim _{t \rightarrow \infty}\left(E_{i}(t)-P_{i}(t)\right)=0
$$

for $i=1,2$. In a belief affirming process players' expected payoffs and average realized payoffs coincide in the long run.

\subsection{CFP Convergence in Zero-Sum Games}

The aim of this chapter is to prove the following.

Theorem 6.3.1. CFP in zero-sum games converges to Nash equilibrium.

The proof requires two simple lemmas. The first is due to Monderer et al. (1997). For completeness, we include the proof.

Lemma 6.3.2 (Monderer et al. 1997). Every CFP is belief affirming.

Proof. Each function $g_{i}:[1, \infty) \rightarrow \mathbb{R}, i \in I$, defined as

$$
g_{i}(t):=\int_{0}^{t} e^{i} A y(\tau) d \tau,
$$

is continuous on $[1, \infty)$. Since $t E_{1}(t)=\max \left\{g_{i}(t) \mid i \in I\right\}$ for all $t \geq 1$, also $t E_{1}(t)$ is continuous on $[1, \infty)$. The function $t P_{1}(t)$ is also continuous on $[1, \infty)$; it follows that $t E_{1}(t)-t P_{1}(t)$ is continuous on $[1, \infty)$.

Suppose $(x(\cdot), y(\cdot))$ is continuous at $t$. Since $x(\cdot)$ and $y(\cdot)$ are by definition pure strategies, there is an $\varepsilon>0$ such that $x(\tau)$ and $y(\tau)$ are constant for all $\tau \in(t-\varepsilon, t+\varepsilon)$. This implies that the derivatives of $t E_{1}(t)$ and $t P_{1}(t)$ at $t$ are both equal to $x(t) A y(t)$. It follows that $t E_{1}(t)-t P_{1}(t)$ is constant on each interval on which $(x(\cdot), y(\cdot))$ is continuous. Continuity of $t E_{1}(t)-t P_{1}(t)$ on $[1, \infty)$ then implies

$$
t E_{1}(t)-t P_{1}(t)=K,
$$


for all $t \in[1, \infty)$, where $K:=E_{1}(1)-P_{1}(1)$. Thus,

$$
\lim _{t \rightarrow \infty}\left(E_{1}(t)-P_{1}(t)\right)=\lim _{t \rightarrow \infty} \frac{1}{t}\left(t E_{1}(t)-t P_{1}(t)\right)=\lim _{t \rightarrow \infty} \frac{K}{t}=0 .
$$

A similar argument applies to player 2 .

Lemma 6.3.3. $\lim _{t \rightarrow \infty} W(p(t), q(t))=0$.

Proof. It follows from Lemma 6.3.2 that CFP is belief affirming. This implies

$$
\begin{aligned}
0 & =\lim _{t \rightarrow \infty}\left(E_{1}(t)-P_{1}(t)\right)+\lim _{t \rightarrow \infty}\left(E_{2}(t)-P_{2}(t)\right) \\
& =\lim _{t \rightarrow \infty}\left(E_{1}(t)-P_{1}(t)+E_{2}(t)-P_{2}(t)\right) \\
& =\lim _{t \rightarrow \infty}\left(E_{1}(t)+E_{2}(t)\right) \\
& =\lim _{t \rightarrow \infty} W(p(t), q(t)),
\end{aligned}
$$

where the third equality follows from $P_{1}(t)=-P_{2}(t)$ for all $t$, and the fourth from $W(p(t), q(t))=E_{1}(t)+E_{2}(t)$ for all $t$.

Proof of Theorem 6.3.1 Take some $\left(p^{*}, q^{*}\right) \in \Omega$ and a sequence of times $\left(t_{k}\right)_{k=1}^{\infty}$ such that $\lim _{k \rightarrow \infty}\left(p\left(t_{k}\right), q\left(t_{k}\right)\right)=\left(p^{*}, q^{*}\right)$. Note that the existence of such a sequence is implied by $\left(p^{*}, q^{*}\right) \in \Omega$. Since $W$ is continuous in $(p, q)$ we have

$$
\lim _{k \rightarrow \infty} W\left(p\left(t_{k}\right), q\left(t_{k}\right)\right)=W\left(p^{*}, q^{*}\right) .
$$

By Lemma 6.3.3 it follows that $W\left(p^{*}, q^{*}\right)=0$, which by Theorem 6.2.1 implies $\left(p^{*}, q^{*}\right) \in N E(A)$. Hence, $\Omega \subseteq N E(A)$.

Remark 6.3.4. From Equation (6.1) it follows that $\left(E_{1}(t)-P_{1}(t)\right)$ converges at rate $\frac{1}{t}$; this also holds for player 2 . It follows that $(p(t), q(t))$ converges to equilibrium at a rate of $\frac{1}{t}$, confirming Harris' (1998) result.

Remark 6.3.5. Our proof of Theorem 6.3.1 seems to be closer to Brown's heuristic argument than the one usually presented in the literature. For instance, Brown (1951, pp. 375) says: ${ }^{3}$ "In the system of differential equations the convergence rests on the fact that $t \bar{V}(t)$ and $t \underline{V}(t)$ maintain a constant difference between them."

\footnotetext{
${ }^{3}$ Here, $\bar{V}(t) \equiv E_{1}(t)$ and $\underline{V}(t) \equiv-E_{2}(t)$.
} 


\subsection{DFP in Zero-Sum Games}

A Discrete Fictitious Play (DFP) in $A$ is a sequence $(x(t), y(t))$ in $\tilde{I} \times \tilde{J}, t \in \mathbb{N}$, with $(x(0), y(0)) \in \tilde{I} \times \tilde{J}$ and for all $t \in \mathbb{N} \backslash\{0\}$,

$$
(x(t), y(t)) \in \beta\left(\frac{1}{t} \sum_{\tau=0}^{t-1}(x(\tau), y(\tau))\right) .
$$

The sequence of beliefs $(p(t), q(t)): \mathbb{N} \backslash\{0\} \rightarrow \Delta^{m} \times \Delta^{n}$, is given by

$$
\frac{1}{t} \sum_{\tau=0}^{t-1}(x(\tau), y(\tau))
$$

Like before, we define $E_{1}(t):=x(t) A q(t), E_{2}(t):=-p(t) A y(t)$,

$$
P_{1}(t):=\frac{1}{t} \sum_{\tau=0}^{t-1} x(\tau) A y(\tau), \text { and } P_{2}(t):=-P_{1}(t) .
$$

The following result was proven by Robinson (1951).

Theorem 6.4.1 (Robinson 1951). DFP in zero-sum games converges to Nash equilibrium.

A DFP satisfies the condition of infrequent switching ${ }^{4}$ if

$$
\lim _{t \rightarrow \infty} \frac{1}{t} \sum_{\tau=1}^{t} M_{i}(\tau)=0
$$

for $i=1,2$, where

$$
M_{1}(t):=\left\{\begin{array}{ll}
1 & \text { if } x(t) \neq x(t-1) \\
0 & \text { otherwise. }
\end{array} \text { and } \quad M_{2}(t):= \begin{cases}1 & \text { if } y(t) \neq y(t-1) \\
0 & \text { otherwise }\end{cases}\right.
$$

for all $t \in \mathbb{N} \backslash\{0\}$. Monderer et al. (1997), and Fudenberg and Levine (1994) established the following result.

Lemma 6.4.2. Every DFP satisfying infrequent switching is belief affirming.

Hence, if the DFP process satisfies infrequent switching, then its convergence in zero-sum games follows along the lines of the previous section. Whether DFP in zero-sum games satisfies infrequent switching remains an open question.

\footnotetext{
${ }^{4}$ Monderer et al. (1997) call DFP's that satisfy infrequent switching "smooth" Fictitious Plays.
} 


\section{Bibliography}

Allais, M. (1953). Le comportement de l'homme rationnel devant le risque: critique des postulats et axiomes de l'école americaine. Econometrica 21(4), 503-546.

Anscombe, F. J. and R. J. Aumann (1963). A definition of subjective probability. Annals of Mathematical Statistics 34(1), 199-205.

Arrow, K. J. (1964). The role of securities in the optimal allocation of riskbearing. Review of Economic Studies 31(2), 91-96.

Arrow, K. J. (1971). Essays in the theory of risk bearing. Markham Pub. Co.

Berden, C. and H. Peters (2006). On the effect of risk aversion in bimatrix games. Theory and Decision 60(4), 359-370.

Berejikian, J. D. (2002). Model building with prospect theory: A cognitive approach to international relations. Political Psychology 23(4), 759786.

Bernoulli, D. (1954). Exposition of a new theory on the measurement of risk. Econometrica 22(1), 22-23. English translation of Bernoulli, D. (1738). Specimen theoriae novae de mensura sortis. Papers of the Imperial Academy of Sciences in Petersburg 5, 175 - 192.

Binmore, K., A. Rubinstein, and A. Wolinsky (1986). The Nash bargaining solution in economic modeling. The RAND Journal of Economics 17(2), 176-188.

Brown, G. W. (1949). Some notes on computation of games solutions. RAND report P-78, The RAND Corporation, Santa Monica, Califor- 
nia.

Brown, G. W. (1951). Iterative solution of games by fictitious play. In T. C. koopmans (Ed.), Activity Analysis of Production and Allocation, Chapter 24, pp. 374-376. Wiley, New York.

Butler, C. K. (2007). Prospect theory and coercive bargaining. Journal of Conflict Resolution 51(2), 227-250.

Camerer, C. F. (2002). Prospect theory in the wild: evidence from the field. In D. Kahneman and A. Tversky (Eds.), Choices, Values, and Frames, pp. 288-301. Cambridge University Press.

Chateauneuf, A. and J.-M. Tallon (2002). Diversification, convex preferences and non-empty core in the choquet expected utility model. Economic Theory 19(3), 509-523.

Compte, O. and P. Jehiel (2003). Bargaining with reference dependent preferences. mimeo CERAS, ENPC, Paris.

Crawford, V. P. (1990). Equilibrium without independence. Journal of Economic Theory 50(1), 127-154.

Debreu, G. (1959). Theory of Value: An Axiomatic Analysis of Economic Equilibrium. Cowles Foundation Monographs Series. Yale University Press, New Haven.

Debreu, G. and T. C. Koopmans (1982). Additively decomposed quasiconvex functions. Mathematical Programming 24(1), 1-38.

Dekel, E., Z. Safra, and U. Segal (1991). Existence and dynamic consistency of Nash equilibrium with non-expected utility preferences. Journal of Economic Theory 55(2), 229-246.

Eichberger, J. and D. Kelsey (1999). Non-additive beliefs and strategic equilibria. Games and Economic Behavior 30(2), 183-215.

Ergin, H. and F. Gul (2009). A theory of subjective compound lotteries. Journal of Economic Theory 144(3), 899-929.

Fershtman, C. (1996). On the value of incumbency: Managerial reference points and loss aversion. Journal of Economic Psychology 17(2), 245257.

Fishburn, P. C. (1970). Utility theory for decision making. New York: Wiley. 
Fudenberg, D. and D. K. Levine (1995). Consistency and cautious fictitious play. Journal of Economic Dynamics and Control 19(4), 10651089.

Gilboa, I. (1987). Expected utility with purely subjective non-additive probabilities. Journal of Mathematical Economics 16(1), 65-88.

Gilboa, I. and D. Schmeidler (1989). Maxmin expected utility with nonunique prior. Journal of Mathematical Economics 18(2), 141-153.

Grant, S., A. Kajii, and B. Polak (2001). Şthird down with a yard to goT: recursive expected utility and the dixitÜskeath conundrum. Economics Letters 73(3), 275-286.

Hammond, P. J. (1988). Consequentialist foundations for expected utility. Theorey and Decision 25, 25-78.

Hansen, L. P. and T. J. Sargent (2001). Robust control and model uncertainty. American Economic Review 91, 60-66.

Hardy, G. H., J. E. Littlewood, and G. Pòlya (1934). Inequalities (second ed.). The Cambridge Mathematical Library. Cambridge University Press, Cambridge, UK.

Harris, C. (1998). On the rate of convergence of continuous-time fictitious play. Games and Economic Behavior 22(2), 238-259.

Harsanyi, J. C. and R. Selten (1972). A generalized Nash solution for twoperson bargaining games with incomplete information. Management Science 18(5), 80-106.

Hendon, E., H. J. Jacobsen, and B. Sloth (1996). The one-shot-deviation principle for sequential rationality. Games and Economic Behavior 12(2), 274-282.

Hershey, J. C., H. C. Kunreuther, and P. J. H. Schoemaker (1982). Sources of bias in assessment procedures for utility functions. Management Science 28(8), 936-954.

Hofbauer, J. (1994). Stability for the best-response dynamics. Mimeo Institut für Mathematik, Universität Wien.

Hofbauer, J. and S. Sorin (2006). Best-response dynamics for continuous zero-sum games. Discrete and Dynamical Systems-Series B 6(1), 215224.

Kahneman, D. and A. Tversky (1979). Prospect theory: an analysis of decision under risk. Econometrica 47(2), 263-291. 
Kalai, E. (1977). Nonsymmetric Nash solutions and replications of 2person bargaining. International Journal of Game Theory 6(3), 129133.

Kalai, E. and M. Smorodinsky (1975). Other solutions to Nash's bargaining problem. Econometrica 43(3), 513-518.

Karni, E. and Z. Safra (1989). Dynamic consistency, revelations in auctions and the structure of preferences. Review of Economic Studies 56, 421-434.

Kihlstrom, R. E., A. E. Roth, and D. Schmeidler (1981). Risk aversion and solutions to Nash's bargaining problem. In O. Moeschlin and D. Pallaschke (Eds.), Game theory and mathematical economics, pp. 65-72. North-Holland Publishing Company.

Klibanoff, P., M. Marinacci, and S. Mukherji (2005). A smooth model of decision making under ambiguity. Econometrica 73(6), 1849-1892.

Köbberling, V. and H. Peters (2003). The effect of decision weights in bargaining problems. Journal of Economic Theory 110(1), 154-175.

Köbberling, V. and P. P. Wakker (2003). Preference foundations for nonexpected utility: A generalized and simplified technique. Mathematics of Operations Research 28(3), 395 - 423.

Kreps, D. M. and E. L. Porteus (1978). Temporal resolution of uncertainty and dynamic choice theory. Econometrica 46(1), 185-200.

Krishna, V. and T. Sjöström (1997). In S. Hart and A. Mas-Colell (Eds.), Cooperation: Game-theoretic Approaches, Volume 55 of NATO-ASI Series $F$, pp. 257-273. Springer, Berlin.

Li, D. (2007). Bargaining with history-dependent preferences. Journal of Economic Theory 136(1), 695-708.

Maccheroni, F., M. Marinacci, and A. Rustichini (2006). Ambiguity aversion, robustness, and the variational representation of preferences. Econometrica 74(6), 1447-1498.

Machina, M. J. (1987). Choice under uncertainty: problems solved and unsolved. Journal of Economic Perspectives 1(1), 121-154.

Machina, M. J. (1989). Dynamic consistency and non-expected utility models of choice under uncertainty. Journal of Economic Literature 27(4), 1622-1688. 
Monderer, D., D. Samet, and A. Sela (1997). Belief affirming in learning processes. Journal of Economic Theory 73(2), 438-452.

Myerson, R. B. (1991). Game theory: Analysis of conflict. Harvard University Press, Cambridge, Massachusetts.

Nash, J. F. (1950a). The bargaining problem. Econometrica 18(2), 155162.

Nash, J. F. (1950b). Non-cooperative games. Ph. D. thesis, Princeton University.

Nau, R. F. (2006). The shape of incomplete preferences. Annals of Statistics 34(5), 2430-2448.

Neilson, W. S. (1993). Ambiguity aversion: An axiomatic approach using second order probabilities. Mimeo, Dept. of Economics, University of Tennessee, TN.

Peters, H. and S. Tijs (1984). Individually monotonic bargaining solutions for $n$-person bargaining games. Methods of Operations Research 51, 377-384.

Peters, H. and E. van Damme (1991). Characterizing the Nash and Raiffa bargaining solutions by disagreement point axioms. Mathematics of Operations Research 16(3), 447-461.

Pratt, J. W. (1964). Risk aversion in the small and in the large. Econometrica $32,122-136$.

Rabin, M. (2000). Risk aversion and expected-utility theory: A calibration theorem. Econometrica 68(5), 1281-1292.

Raiffa, H. (1953). Arbitration schemes for generalized two-person games. In H. W. Kuhn, A. W. Tucker, and M. Dresher (Eds.), Contributions to the Theory of Games II, Annals of Mathematics Studies 28, pp. 361387. Princeton University Press.

Robinson, J. (1951). An interative method of solving a game. Annals of Mathematics 54(2), 296-301.

Roth, A. E. (1977). Individual rationality and Nash's solution to the bargaining problem. Mathematics of Operations Research 2(1), 64-65.

Roth, A. E. (1979). An impossibility result concerning $n$-person bargaining games. International Journal of Game Theory 8(3), 129-132. 
Roth, A. E. (1985). A note on risk aversion in a perfect equilibrium model of bargaining. Econometrica 53(1), 207-211.

Rubinstein, A. (1982). Perfect equilibrium in a bargaining model. Econometrica 50(1), 97-109.

Safra, Z. and I. Zilcha (1993). Bargaining solutions without the expected utility hypothesis. Games and Economic Behavior 5(2), 288-306.

Savage, L. J. (1954). The Foundations of Statistics (second ed.). Wiley, New York. 1972, Dover Publications, New York.

Schmeidler, D. (1989). Subjective probability and expected utility without additivity. Econometrica 57(3), 571-587.

Schoemaker, P. J. H. (1982). The expected utility model: Its variants, purposes, evidence and limitations. Journal of Economic Literature 20(2), $529-563$.

Shalev, J. (2000). Loss aversion equilibrium. International Journal of Game Theory 29(2), 269-287.

Shalev, J. (2002). Loss aversion and bargaining. Theory and Decision 52(3), 201-232.

Shamma, J. S. and G. Arslan (2004). Unified convergence proofs of continuous-time fictitious play. IEEE Transactions on Automatic Control 49(7), 1137-1142.

Ståhl, I. (1972). Bargaining theory. Economic Research Institute, Stockholm School of Economics (Stockholm).

Tversky, A. and D. Kahneman (1981). The framing of decisions and the psychology of choice. Science, New Series 211(4481), 453-458.

von Neumann, J. (1928). Zur theorie der gesellschaftsspiele. Mathematische Annalen 100(1), 295-320.

von Neumann, J. and O. Morgenstern (1944). Theory of Games and Economic Behavior. Princeton University Press.

Wakker, P. P. (1993). Unbounded utility for savage's "foundations of statistics," and other models. Mathematics of Operations Research 18, 446485.

Wakker, P. P. (2009). Prospect theory for risk and ambiguity. Cambridge, UK: Cambridge University Press. 
Wakker, P. P. and D. Deneffe (1996). Eliciting von neumann-morgenstern utilities when probabilities are distorted or unknown. Management Science 42(8), 1131-1150.

Yaari, M. (1969). Some remarks on measures of risk aversion and on their uses. Journal of Economic Theory 1(3), 315 - 329.

Zermelo, E. (1913). Über eine anwendung der mengenlehre auf die theorie des schachspiels. In E. W. Hobson and A. E. Love (Eds.), Proceedings of the Fifth International Congress of Mathematicians II, Cambridge, pp. 501-504. Cambridge University Press. 



\section{Nederlandse Samenvatting}

Dit proefschrift behandelt onderwerpen in de besliskunde, de speltheorie, en het raakvlak tussen deze beide vakgebieden. Het onderdeel van de besliskunde dat we beschouwen in deze tekst, beslissen onder onzekerheid, onderzoekt hoe individuen beslissingen en keuzes maken in een onzekere wereld, en hoe deze worden beïnvloed door de gedragssamenstelling van degene die de beslissing neemt. Dit wordt misschien best geillustreerd door het klassieke voorbeeld van Bernoulli (1738), die de vraag stelde tegen welke prijs men een lot uit de loterij dient te verkopen dat met gelijke kans $€$ 20.000 of $€ 0$ oplevert. Dat wil zeggen, welk zeker geldbedrag zou de bezitter van een dergelijk lot als een passende vergoeding beschouwen voor de kans $€ 20.000$ te winnen, en het risico met niets te eindigen. Uiteraard verandert het probleem als we niet langer met zekerheid weten met welke kans de lotto wordt gewonnen, of wanneer men niet de verkoper is van zo'n lot, maar de koper.

Dagelijks nemen wij ontelbare beslissingen, zowel kleine als grote. Doe ik er goed aan mij te verzekeren tegen fietsdiefstal? Moet ik de auto nemen naar mijn werk, in de wetenschap dat de fiets trager is, maar veiliger? Moet ik vandaag stoppen met roken, wetende dat dit mij kan behoeden voor potentiële gezondheidsproblemen in de toekomst? Door het onderzoeken van problemen zoals dit van Bernoulli, tracht de besliskunde de systemen aan het licht te brengen die aan onze beslissingen ten grondslag liggen. Zij bezet dan ook een belangrijke plaats in verschillende humane wetenschappen, zoals daar zijn psychologie, sociologie, en economie.

Beslissen onder onzekerheid beperkt zich tot het onderzoeken van keuzeproblemen van geïsoleerde individuen, en gaat derhalve voorbij aan de 
interactie die inherent is aan het economisch handelen. De uitkomst van de beslissingen die een agent neemt hangt niet enkel af van welke staat van de wereld gerealiseerd wordt, maar tevens van de beslissingen genomen door één of meerdere andere agenten die geconfronteerd worden met exact hetzelfde hetzelfde probleem, zijnde het maken van de optimale keuze in de wetenschap dat de uitkomst afhangt van de combinatie van alle gespeelde strategieën, zowel die van de anderen als die van henzelf. Dergelijke strategische interacties worden bestudeerd in de speltheorie.

Hoewel speltheorie een toepassing vindt in vele wetenschappelijke disciplines, zijn er slechts weinig waarin zij een zo prominente plaats inneemt als in economie. Op een paar losstaande uitzonderingen na, is het gebruik van speltheorie in de economie begonnen met de publicatie van von Neumann en Morgenstern's boek Theory of Games and Economic Behavior. Hier bekomen zij een hoogst elegante integratie van de besliskundige principes van Bernoulli met de speltheoretische concepten die op dat moment in voege waren. De theorie van verwacht nut die zij hiervoor aanwendden, bleef evenwel niet lang zonder kritiek. Reeds in de jaren 50 leidde Allais een experiment waarmee werd aangetoond dat individuen keuzes maken die hiermee niet verenigbaar zijn. Over de jaren heen doken er meer en meer paradoxen en puzzels op die de theorie van verwacht nut in vraag zouden stellen; de nood aan een alternatief beslismodel drong zich op.

Een belangrijk alternatief model - genaamd prospecttheorie - werd ontwikkeld door Kahneman en Tversky (1979). Zij behielden de basis van het verwacht nutsmodel, maar verrijkten dit met een aantal gedragskenmerken, waaronder verliesaversie. Verliesaversie is gebaseerd op het idee dat bij het nemen van beslissingen, een mogelijk verlies in het algemeen zwaarder doorweegt dan een potentiële winst van dezelfde grootte. Als we weten dat Kahneman en Tversky's beslismodel dergelijke besliskundige eigenaardigheden in acht neemt, hoeft het niet te verbazen dat het significant beter doet dan haar verwacht nutstegenhanger. Dit succes voor individuele beslissingen voedt het vermoeden dat het invoeren van (elementen van) prospecttheorie in de speltheorie op eenzelfde manier zal leiden tot betere modellen voor strategische interacties. We trachten in dit proefschrift een licht te werpen op het effect van verliesaversie op enkele bekende resultaten uit de speltheorie.

Verliesaversie wordt gemodelleerd met de simpele transformatie van Shalev (2000). Shalev nam aan dat elke speler een bepaald nutsniveau $r$ in gedachten heeft - een verwachting of een vooraf gesteld doel - dat zijn hele 
spectrum van nutsuitkomsten opsplitst in winsten en verliezen. Dit nutsniveau wordt ook het referentiepunt van deze speler genoemd. Tversky en Kahneman (1981) gaven al aan dat elke nutsuitkomst $x$ kan uitgedrukt worden als een afwijking van dit referentiepunt: $x=r+(x-r)$. Kahneman en Tversky's (1979) stelling dat negatieve afwijkingen $(x-r<0)$ belangrijker zijn dan positieve $(x-r \geq 0)$, werd door Shalev gemodelleerd met de elegante transformatie

$$
U(x)= \begin{cases}r+(x-r) & \text { if } x-r \geq 0, \\ r+(1+\lambda) \cdot(x-r) & \text { if } x-r<0\end{cases}
$$

waar de positieve parameter $\lambda$ - de verliesaversiecoëfficiënt - een maat is voor hoe negatief de agent precies staat tegenover verliezen. Om de effecten van verliesaversie op de uitkomst van strategische interacties vast te stellen, hoeven we in principe slechts de mogelijke uitbetalingen van de spelers te corrigeren met Shalevs transformatie. Dit gaat echter voorbij aan het feit dat we de referentiepunten van de spelers niet kennen. Het is duidelijk dat deze de uitkomst van een spel beïnvloeden. Het omgekeerde is echter eveneens waar: welke uitbetalingen een individuele speler als verlies zal beschouwen, en welke als winst, hangt af van hoe deze speler verwacht dat het spel gespeeld zal worden, en dus welke uitbetaling hij denkt te kunnen realiseren. Het besluit dat wij hieruit trekken is dat een correcte integratie van verliesaversie in speltheorie vereist dat het bepalen van spelers' referentiepunten deel uitmaakt van het evenwichtsconcept waarmee het spel wordt gespeeld.

In hoofdstuk 2 onderzoeken we wat dit betekent voor het onderhandelingsspel van Rubinstein (1982) waarin twee spelers een akkoord moeten bereiken over de verdeling van een perfect verdeelbaar goed. De onderhandeling bestaat erin dat ze mekaar om de beurt een voorstel doen, totdat één van de twee akkoord gaat met het aanbod van de ander. Daarop wordt de afgesproken verdeling geïmplementeerd. Een aanbod afslaan leidt met een kleine kans tot het doodlopen, en dus eindigen van de onderhandelingen; in deze situatie krijgen beide spelers niets. Rubinstein toonde aan dat dit spel een uniek Subgame Perfect Evenwicht (SPE) heeft. In dit proefschrift wordt de spelsituatie uitgebreid met de aanname dat beide spelers verliesavers zijn, en dat ieders referentiepunt gelijk is aan het hoogste bod van zijn tegenspeler dat hij in het verleden afgewezen heeft. In dit uitgebreid spel construeren wij een SPE dat we bovendien karakteriseren 
door drie eigenschappen die het gemeen heeft met Rubinsteins verliesneutrale evenwicht. Hoewel een dergelijke karakterisering niet uitsluit dat het spel met verliesaverse spelers nog andere evenwichten heeft, kunnen we wel besluiten dat ons evenwicht het enige is dat gelijkaardig is aan dat van Rubinstein. Het is welbekend dat Rubinsteins evenwicht in de Nash onderhandelingsoplossingsuikomst resulteert, als we de kans op het doodlopen van de onderhandelingen na het afwijzen van een voorstel, naar nul laten convergeren. Wij tonen aan dat het SPE in het spel met verliesaverse spelers op een gelijkaardige manier leidt tot een asymmetrische Nash onderhandelingsoplossingsuitkomst, waarin de sterkte van een spelers onderhandelingspositie invers gerelateerd is aan zijn eigen verliesaversiecoëfficiënt, en positief aan die van zijn tegenstander.

Hoofdstuk 3 onderzoekt de effecten van verliesaversie in axiomatische onderhandelingstheorie. Centraal in deze theorie staat Nash' (1950a) onderhandelingsprobleem. Onderhandelaars dienen samen een akkoord te bereiken over één welbepaald element uit een verzameling van mogelijke uitkomsten, in de wetenschap dat het niet eens raken een uitkomst oplevert die slecht is voor hen allemaal. We nemen aan dat spelers verliesavers zijn, en passen Shalevs (2002) methode van zelfonderhoud toe. Als alle verliesaverse spelers, gegeven een bepaalde onderhandelingsoplossing, vooraf een bepaalde uitbetaling verwachten, en de uitkomst die ze uiteindelijk realiseren gegeven die verwachting is exact gelijk aan die verwachting, dan zeggen we dat deze uitkomst zichzelf onderhoudt voor die welbepaalde onderhandelingsoplossing. Wij tonen aan dat een onderhandelingsprobleem juist één uitkomst heeft die zichzelf onderhoudt onder de Kalai-Smorodinsky onderhandelingsoplossing. Verder definiëren we een klasse van onderhandelingsoplossingen die deze unieke uitkomst implementeert. Deze oplossingsklasse wordt gekarakteriseerd door een drietal standaardaxioma's uit de onderhandelingsliteratuur, en een nieuw axioma dat in dit proefschrift wordt gedefinieerd.

In hoofdstuk 4 bestuderen we drie verschillende verliesaversie evenwichten in bimatrixspelen. Een verliesaversie evenwicht is een Nash evenwicht van een spel waarin beide spelers verliesavers zijn, en waarin hun referentiepunten endogeen zijn aan de evenwichtsberekening. Het eerste evenwicht dat we beschouwen is Shalevs (2000) 'fixed point' verliesaversie evenwicht, waarin het referentiepunt van elke speler exact afhangt van het strategieprofiel dat hij gelooft dat zijn tegenstander zal spelen. Bij het tweede type evenwicht dat we beschouwen, het maximin evenwicht, hangt het refer- 
entiepunt slechts af van de dragers van de evenwichtsstrategie van de tegenstander, en niet langer van de precieze kansen. In het derde evenwichtsconcept, veiligheidswaarde-evenwichten, wordt de waarde van de eigen uitbetalingsmatrix als referentiepunt genomen. Shalev toonde reeds aan dat er steeds een fixed-point verliesaversie-evenwicht bestaat. Wij tonen het algemene bestaan van maximin-evenwichten aan in $m \times 2$ - en $2 \times n$-spelen; met een voorbeeld laten we zien dat dit niet voor algemenere bimatrixspelen geldt. We bewijzen tevens het algemene bestaan van veiligheidswaardeevenwichten. Het hoofdstuk wordt afgesloten met een comparative statics analyse. We leiden een conditie af onder de welke verliesaversie van de tegenstander een goede zaak is.

Hoofdstuk 5 gaat over aversie voor risico en ambiguiteit. Het is welbekend dat het model van verwacht nut de mate van risico-aversie van een agent gelijk stelt aan de concaviteit van zijn nutsfunctie. Hier introduceren we een nieuwe techniek die toelaat de concaviteit van verschillende nutsfuncties - en dus risico-attitudes - met elkaar te vergelijken met slechts een beperkte kennis van kansen of staten van de wereld. Deze techniek, de preferentiemiddelpuntstechniek, is gebaseerd op een discrete versie van marginal rates of substitution, een welbekend concept uit de consumententheorie. Hoewel de preferentiemiddelpuntstechniek wiskundig gezien vrij elementair is, veralgemeent zij niettemin een aantal klassieke resultaten over beslissen onder onzekerheid. Daar het misschien niet helemaal geloofwaardig is dat we risico-attitudes kunnen bestuderen met een slechts zeer beperkte aandacht voor kansen of staten van de wereld, werpt deze techniek tevens een nieuw licht op de descriptieve waarde van nut voor het modelleren van risico- en ambiguïteitsattitudes.

Het proefschrift besluit met een zuiver speltheoretisch resultaat dat in principe los staat van risico- en verliesaversie. Om precies te zijn, we leveren een alternatief bewijs voor het welgekende resultaat dat tweepersoons nulsomspelen gekenmerkt worden door de Fictitious Play-eigenschap. Fictitious Play is een leerproces dat behoort tot de literatuur die het Nash evenwichtsconcept tracht te rechtvaardigen middels het argument dat begrensd rationele spelers evenwichtsstrategieën kunnen leren spelen. Spelers spelen op ieder moment hun optimale strategieën, gegeven de empirische strategie van de tegenstanders. Als met deze procedure het empirisch strategieprofiel voor een bepaald spel naar een Nash evenwicht convergeert, ongeacht de startcondities, dan zeggen we dat dit spel de Fictitious Play eigenschap heeft. Robinson (1951) toonde reeds aan dat het discrete Fictitious Play 
proces in nulsomspelen naar een Nash evenwicht convergeert. Dat dit ook geldt voor het continue Fictitious Play proces is reeds geweten sinds het werk van Brown (1949). Op een rigoureus bewijs was het echter wachten tot eind jaren negentig (Harris 1998). Wij leveren een alternatief bewijs voor dit resultaat, dat zowel korter als gemakkelijker is dan dit van Harris. 


\section{Curriculum Vitae}

Bram Willy Ingrid Driesen was born on August 9, 1983 in Genk, Belgium. In June 2001, he received his diploma Algemeen Secundair Onderwijs from the Heilig Hartcollege in Maasmechelen, Belgium. In September of that same year he started studying Econometrics at Maastricht University, with specialization in Mathematical Economics. He received his Master's degree in Econometrics \& Operations Research in February 2006. The final thesis was written during a six-month internship at the financial consultant Montesquieu Finance. Upon completion, he started his doctoral research at the Faculty of Economics and Business Administration of Maastricht University. The results thereof are presented in the present thesis. 